\title{
A 11102 392958
}

NBS

PUBLICATIONS

NBSIR 85-3243

HVACSIM ${ }^{+}$Building Systems and Equipment Simulation Program Users Guide

Daniel R. Clark

William B. May, Jr.

U.S. DEPARTMENT OF COMMERCE

National Bureau of Standards

National Engineering Laboratory

Center for Building Technology

Building Equipment Division

Gaithersburg, MD 20899

September 1985

Emnnsored by:

ional Bureau of Standards

- Navy Civil Engineering Laboratory

. Department of Energy 

Sponsored by:

National Bureau of Standards

U.S. Navy Civil Engineering Laboratory

U.S. Department of Energy

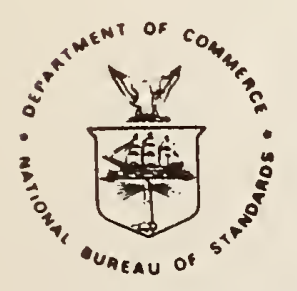

U.S. DEPARTMENT OF COMMERCE, Malcolm Baldrige, Secretary 

HVACSIM $^{+}$is a modular, non-proprietary computer simulation package developed at the National Bureau of Standards. The package consists of a generalpurpose modular simulation program called MODSIM, a library of component models specific to building systems, and a simulation editor called HVACGEN. The latter is used to facilitate the creation and modification of simulation descriptions. HVACSIM ${ }^{+}$is designed to allow detailed simulation of entire building systems or portions of such systems. This includes the heating, ventilating, and air conditioning (HVAC) system, the equipment control system, the conditioned zones within a building, the building shell, and the dynamic interactions among these subsystems.

This document describes the procedures for installing HVACSIM $^{+}$on a particular computer, for setting up a simulation description using HVACGEN, and for running a simulation using MODSIM. A number of example simulations are included to illustrate the procedures and to serve as a tutorial in the use of HVACSIM $^{+}$. A Reference Manual, published separately, describes the main simulation program and the 1 ibrary of component models in more detail. A third document, also published separately, describes the building model supplied with $\operatorname{HVACSIM}^{+}$.

KEY WORDS: building dynamics; building simulation; building system modeling; computer simulation programs; control dynamics; dynamic modeling of building systems; dynamic performance of building systems; dynamic simulations; HVAC system simulations; $\operatorname{HVACSIM}^{+}$ 

Page

LIST OF TABLES .......................................... viii

LIST OF FIGURES .......................................... viii

ACKNOWLEDGMENTS .......................................... $\ldots \ldots$

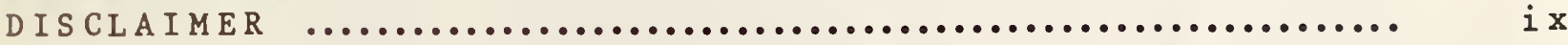

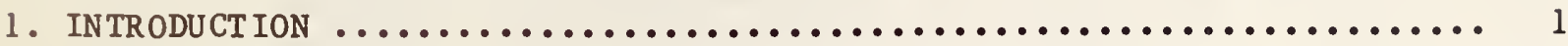

2. DEVELOPMENT OF A SiMULATION $\ldots \ldots \ldots \ldots \ldots \ldots \ldots \ldots \ldots \ldots \ldots \ldots \ldots \ldots \ldots \ldots \ldots$

2.1 Structure of an HVACSIM Simulation ...................... 3

2.2 TYPES Component Library $\ldots \ldots \ldots \ldots \ldots \ldots \ldots \ldots \ldots \ldots \ldots \ldots \ldots \ldots \ldots \ldots \ldots \ldots$

2.3 The System Diagram $\ldots \ldots \ldots \ldots \ldots \ldots \ldots \ldots \ldots \ldots \ldots \ldots \ldots \ldots \ldots \ldots \ldots \ldots \ldots$

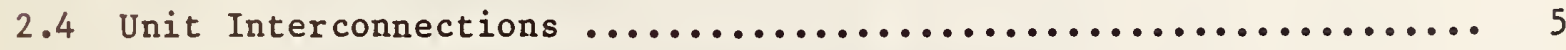

2.5 Grouping Units Into Blocks and Superblocks ................ 6

2.6 Boundary Conditions $\ldots \ldots \ldots \ldots \ldots \ldots \ldots \ldots \ldots \ldots \ldots \ldots \ldots \ldots \ldots$

2.7 Reported Variables ............................... 8

2.8 Work Files and the Model Definition File ................. 8

2.9 Developing Complex Simulations ........................ 9

2.10 Summary of Model Development Procedure ................... 10

3. HVACGEN - THE HVACSIM MODEL CONFIGURATION PROGRAM .............. 11

3.1 Basic Commands: CREATE, VIEW, and EDIT .................. 11

3.2 Control of the Program $\ldots \ldots \ldots \ldots \ldots \ldots \ldots \ldots \ldots \ldots \ldots \ldots \ldots \ldots \ldots \ldots$

3.3 Command/Menu Syntax $\ldots \ldots \ldots \ldots \ldots \ldots \ldots \ldots \ldots \ldots \ldots \ldots \ldots \ldots \ldots \ldots \ldots$

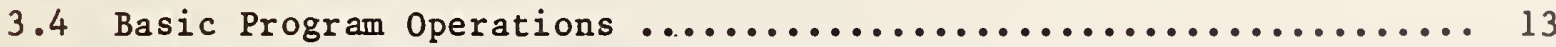

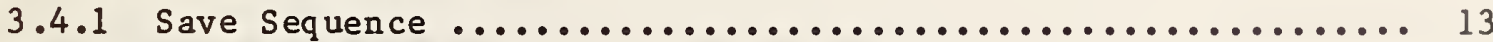

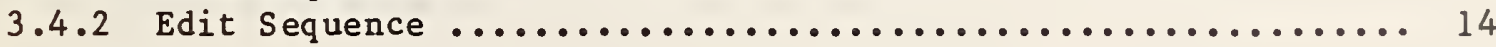

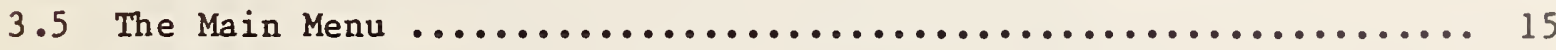

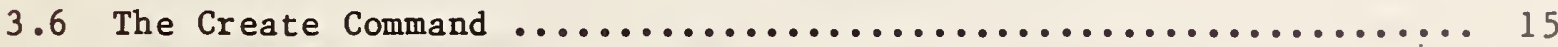

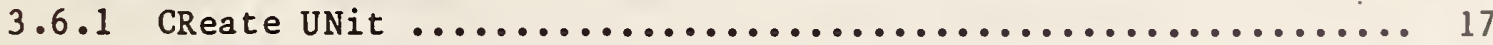

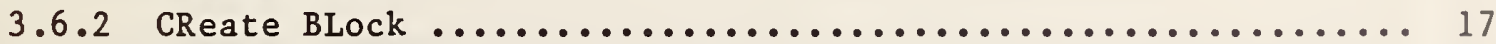

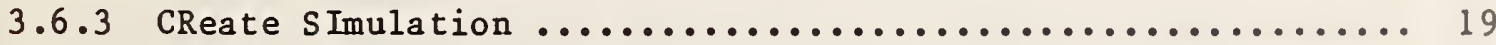

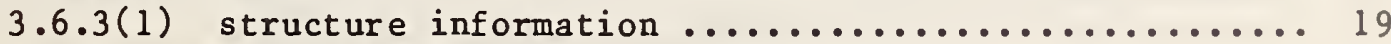

$3.6 .3(2)$ simulation control information ................ 20 
3.7 The View Command ................................... 22

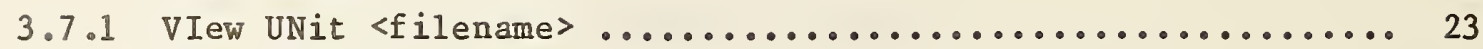

3.7 .2 VIew BLock <filename $\ldots \ldots \ldots \ldots \ldots \ldots \ldots \ldots \ldots \ldots \ldots \ldots, 23$

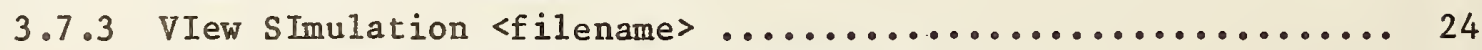

$3.7 .3(1)$ VIew SImulation $\langle$ fil ename $\rangle$ ALI $\ldots \ldots \ldots \ldots \ldots \ldots, 24$

3.7.3(2) VIew SImulation <filename> STructure ............ 24

3.7.3(3) VIew SImulation <filename> VAriable initial values.. 24

3.7.3(4) VIew SImulation <filename> ERror tolerances ....... 24

$3.7 .3(5)$ VIew SImulation $\langle$ filename $\rangle$ BOundary variables ..... 25

3.7.3(6) VIew SImulation <filename> REported variables...... 25

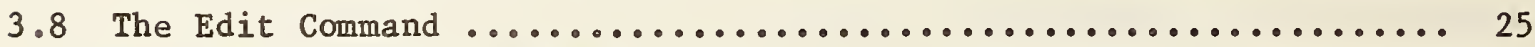

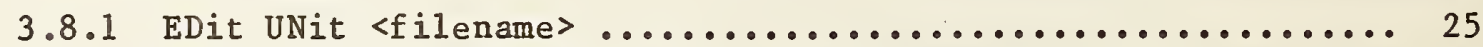

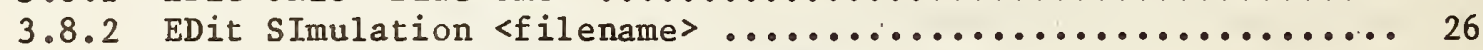

$3.8 .2(1)$ EDit SImulation <filename $>$ TItle $\ldots \ldots \ldots \ldots \ldots \ldots . . \ldots$

$3.8 .2(2)$ EDit SImulation $\langle$ filename $\rangle$ STructure $\ldots \ldots \ldots \ldots \ldots .27$

3.8.2(3) EDit SImulation <filename〉 VAriable initial values.. 27

3.8.2(4) EDit SImulation <filename > ERror tolerances ....... 27

$3.8 .2(5)$ EDit SImulation <filename $>$ BOundary variables ...... 29

3.8.2(6) EDit SImulation $\langle$ filename $\rangle$ REported variables...... 30

3.8.2(7) EDit SImulation <filename > INsert or replace ...... 31

$3.8 .2(7 \mathrm{~A})$ insert unit $\ldots \ldots \ldots \ldots \ldots \ldots \ldots \ldots \ldots \ldots \ldots \ldots \ldots \ldots \ldots \ldots \ldots, 31$

$3.8 .2(7 \mathrm{~B})$ insert block ......................... 32

$3.8 .2(7 \mathrm{C})$ replace unit $\ldots \ldots \ldots \ldots \ldots \ldots \ldots \ldots \ldots \ldots \ldots, 34$

$3.8 .2(8)$ EDit SImulation $\langle$ filename $>$ DElete $\ldots \ldots \ldots \ldots \ldots \ldots . . . \ldots$

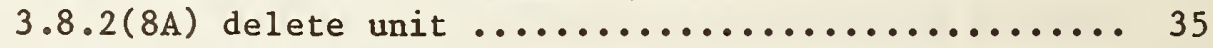

$3.8 .2(8 \mathrm{~B})$ delete block ........................ 35

$3.8 .2(9)$ EDit SImulation <filename > SAve block ............... 36

3.9 The Help Command $\ldots \ldots \ldots \ldots \ldots \ldots \ldots \ldots \ldots \ldots \ldots \ldots \ldots, 36$

3.10 Producing a Model Definition File $\ldots \ldots \ldots \ldots \ldots \ldots \ldots \ldots \ldots . . \ldots \ldots$

4. PREPARING AND RUNNING SIMULATIONS USING HVACSIM ${ }^{+} \ldots \ldots \ldots \ldots \ldots \ldots . . \ldots$

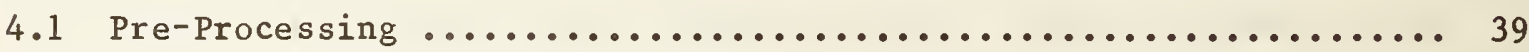

4.1 .1 The Model Definition File ......................... 39

4.1 .2 The Boundary Variable File ......................... 39

4.1 .3 The Initialization File $\ldots \ldots \ldots \ldots \ldots \ldots \ldots \ldots \ldots \ldots \ldots, 40$

4.1.4 The Conduction Transfer Function File $\ldots \ldots \ldots \ldots \ldots \ldots \ldots, 40$

4.1 .5 The Weather Data File $\ldots \ldots \ldots \ldots \ldots \ldots \ldots \ldots \ldots \ldots \ldots, 40$

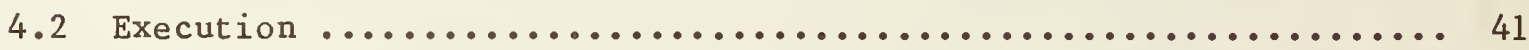

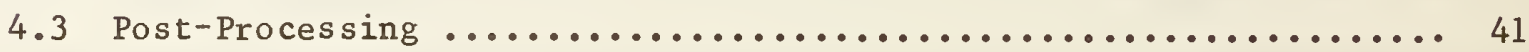

5. INSTALLATION OF THE HVACSIM PROGRAMS $\ldots \ldots \ldots \ldots \ldots \ldots \ldots \ldots \ldots \ldots \ldots$

5.1 HVACS IM Distribution Files $\ldots \ldots \ldots \ldots \ldots \ldots \ldots \ldots \ldots \ldots \ldots, 43$

5.2 Installation Process .............................. 44

5.2.1 HVACSIM Source Code Compilation and Linkage ............ 44 
5.2 .2 SLIMCON Source Code Compilation and Linkage ............ 45

5.2 .3 HVACGEN Source Code Compilation and Linkage ............ 45

5.3 Description of HVACGEN Subroutines .................... 46

5.3 .1 HVACGEN1 - main module ......................... 46

5.3 .2 HVACGEN2 - create module ........................ 47

5.3 .3 HVACGEN3 - file control module ..................... 48

5.3 .4 HVACGEN4 - view module ........................... 49

5.3 .5 HVACGEN5 - edit (changes) module .................... 49

5.3 .6 HVACGEN6 - edit (insert/delete/save) module ............ 50

5.4 Description of SLIMCON Subroutines .................... 51

5.5 Increasing or Decreasing the Simulation Size Capacity ........ 51

6. EXAMPLES OF MODEL DEVELOPMENT, CONFIGURATION, AND EXECUTION ....... 52

6.1 Example 1: Pipe and Valve ......................... 52

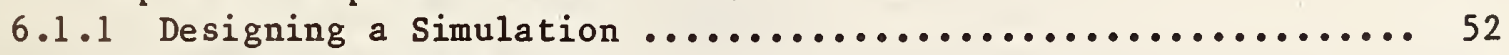

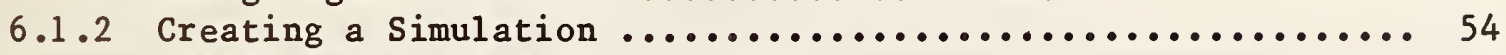

6.1 .3 Creating the Model Definition File .................. 55

6.1 .4 Preparing the Boundary Value File ................... 55

6.1 .5 Running a Simulation ............................ 56

6.1 .6 Discussion of Simulation Results $\ldots \ldots \ldots \ldots \ldots \ldots \ldots \ldots \ldots \ldots \ldots \ldots$

Listing 6.1-1 Interactive HVACGEN session to create EXAMPLE1 .... 59

Listing 6.1-2 EXAMPLEl.SIM: Simulation Work File ............ 67

Listing $6.1-3$ SLIMCON Tabular Output .................... 68

Listing 6.1-4 EXAMPLE1.DAT: Model Definition File ............ 69

Listing $6.1-5$ BOUNDARY.DAT: Boundary Variable File ........... 70

Listing $6.1-6$ Starting a Simulation Using HVACSIM ........... 71

Listing $6.1-7$ INITOUT.DAT: Final state Output File ........... 75

Listing 6.1-8 MODSUM.DAT: Simulation Summary Output File ...... 75

Listing $6.1-9$ MODOUT.DAT: Simulation Data Output File ......... 80

6.2 Example 2: Heating Coil Control Loop, Variable Inlet Water Temp. 90

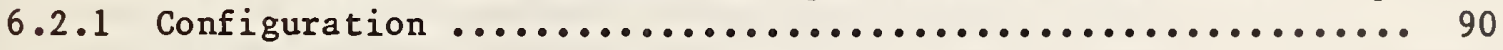

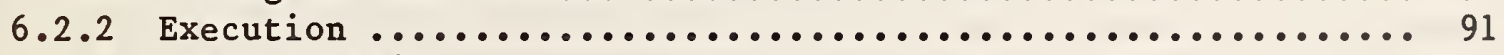

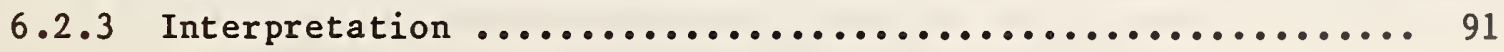

Listing 6.2-1 Summary of EXAMPLE2 Simulation Configuration ...... 94

Listing 6.2-2 EXAMPLE2.SIM: Simulation Work File ............ 100

Listing 6.2-3 Boundary Variable File for Example $2 \ldots \ldots \ldots \ldots . . . .101$

Listing 6.2-4 Initialization File for Example $2 \ldots \ldots \ldots \ldots \ldots \ldots$

Listing 6.2-5 A Portion of the Simulation Output File MODOUT.DAT . 102

6.3 Example 3: Cooling Coil Control Loop, Variable Water Flow Rate . 107

Listing 6.3-1 Boundary Variable File for Example $3 \ldots . . . . . . .109$

Listing 6.3-2 Simulation Configuration Summary from MODSUM.DAT ... 109

Listing 6.3-3 Partial Simulation Results from MODSUM.DAT ....... 113 
6.4 Example 4: Air Handling Unit Without Heat Exchangers ........ 118

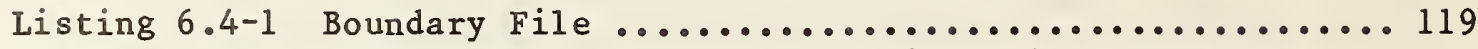

Listing 6.4-2 MODSUM.DAT: Simulation Configuration and Results .. 119

6.5 Example 5: Air Handler With Flow-Controlled Cooling Coil ...... 127

Listing 6.5-1 MODSUM.DAT: Simulation summary and out put ....... 130

6.6 Example 6: Single Zone Air Handler With Cooling Coil ........ 140

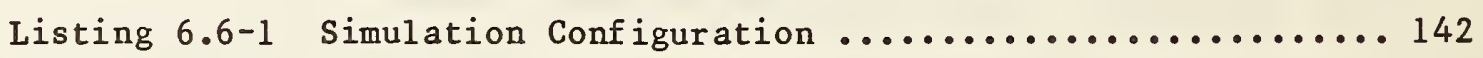

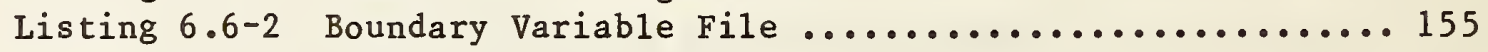

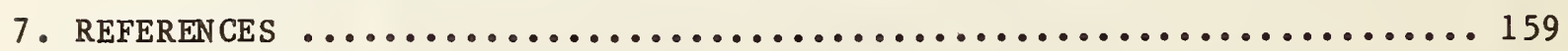

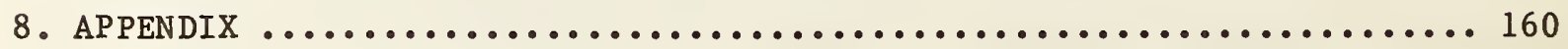

\section{TABLES}

Table 2-1. Categories of Variables .......................... 4

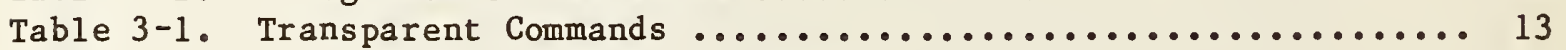

\section{FIGURES}

Figure 3-1. Figure 3-2. Figure 3-3. Figure 6.1-1. Figure 6.1-2. Figure 6.1-3. Figure 6.1-4. Figure 6.2-1. Figure 6.2-2. Figure 6.2-3.

Figure 6.3-1. Figure 6.3-2.

Figure 6.3-3.

Figure 6.3-4.

Figure 6.4-1. Figure 6.5-1. Figure 6.5-2. Figure 6.6-1. Figure 6.6-2. Figure 6.6-3. Figure 6.6-4.
Schematic Diagram of Major Commands of HVACGEN ....... 16 Menu Flow Path for Editing Boundary Variables ........ 28 Menu Flow Path for Editing Reported Variables ........ 29

Example 1: System Schematic Diagram ............... 58

Example 1: Information Flow Diagram .............. 58

Example 1: Control Valve Response ................ 88

Example 1: Temperature Response .................. 89

Constant Flow, Variable Temperature Control System ..... 93

System Schematic for Heating Coil Control Loop ........ 93 Controlled Variable (Air Temperature) Response to

Controller Set Point Step Changes ................. 106

System Schematic for Cooling Coil Control Loop ........ 108 Controlled Variable (Air Temperature) Response to Inlet

Air Temperature Step Changes: No Oscillations ....... 115 Controlled Variable (Air Temperature) Response to Inlet Air Temperature Step Changes: Damped Oscillations .... 116 Controlled Variable (Air Temperature) Response to Inlet

Air Temperature Step Changes: Undamped Oscillations .. 117 Air Handling Unit Without Heat Exchangers: Schematic ... 119 Air Handling Unit With Cooling Coil Control Loop ....... 129 Example 5: Simulation Startup Transient ............. 139 Single-Zone Air Handler With Cooling Coil ............ 141 Example 6: Time Dependent Simulation Inputs .......... 156 Example 6: Temperature Response ................... 157

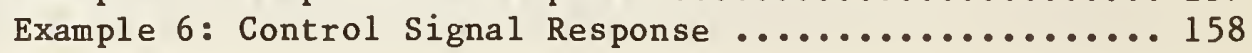




\section{ACKNOWLEDGMENTS}

A number of people have been involved in the development of the HVACSIM ${ }^{+}$ package. Dr. C. Ray Hill conceived and implemented the original main simulation program, MODSIM, during a year spent at the National Bureau of Standards (NBS) as a research associate. He also developed an initial library of component models and a simple precursor to what is now HVACGEN for configuring simulations.

MODSIM was subsequently revised and improved by Dr. Cheol Park and Daniel R. Clark of NBS and renamed HVACSIM. The original component model library was improved and expanded by Daniel R. Clark and contributions to the component models were made by Scott Hildebrand, David Harris, Chris Rasmussen, and Juan Lopez, who were visiting workers at NBS.

The HVACGEN front end program was designed by William B. May of NBS and David Harris, based on the original simple front end program developed by Dr. Hill. David Harris did all of the original program development.

The entire HVACSIM ${ }^{+}$development project was directed by Dr. George E. Kelly of NBS. Financial support for the project from NBS, the U.S. Naval Civil Engineering Laboratory, and the U.S. Department of Energy is gratefully acknow 1edged.

\section{DIS CLA IMER}

The program described in this report is furnished by the government and is accepted and used by any recipient with the express understanding that the United States Government makes no warranty, expressed or implied, concerning the accuracy, completeness, reliability, usability, or suitability for any particular purpose of the information and data contained in this program or furnished in connection therewith, and the United States shall be under no liability whatsoever to any person by reason of any use made thereof. This program belongs to the government. Therefore, the recipient further agrees not to assert any proprietary rights therein or to represent this program to anyone as other than a government program. 

HVACSIM ${ }^{+}$is a non-proprietary building systems simulation computer program developed at the National Bureau of Standards (NBS). This program is intended to simulate the heating, ventilating, and air conditioning (HVAC) system, the equipment control system, the building shell, the building spaces, and the dynamic interaction among these systems. The name is derived from the phrase HVAC SIMulation plus (+) other systems. The program employs advanced equation solving techniques and a hierarchical, modular approach to simulate the dynamic performance of the entire building/HVAC/control system.

This document is intended to serve as a guide for the installation and use of the HVACSIM ${ }^{+}$program. The users guide and one other document [1] contain information to install the $\operatorname{HVACSIM}^{+}$package on a computer, set up simulations, run simulations, interpret the simulation results and expand the library of component models. The building shell model included in the HVACSIM ${ }^{+}$package is the subject of a third document [2].

The HVACSIM ${ }^{+}$package consists primarily of two large programs and one small one. The central program is the main simulation program which takes information from several input files and produces output files containing simulation results. The second program is a "front end" program which is used to configure a simulation to be run by the main simulation program. The small program is used to translate information generated by the configuration program to a form which can be directly used by the main simulation program. Three additional small, independent programs are provided as pre-processors for use in conjunction with the building model. These programs are described in reference [2].

The main simulation program is based on a core program called MODSIM, which in turn is built around a non-linear equation solving package called SNSQ. In principle, MODSIM can be used to simulate any system which can be represented by a set of discrete components, each of which can be described by a set of non-linear ordinary differential and algebraic equations. The MODSIM program is linked with a library of routines for specific system components. A component routine contains the differential and algebraic equations that describe a component such as a piece of equipment or a length of pipe. MODSIM has been made applicable to the solution of building systems by the inculsion of a library of models of building system components such as fans, ducts, rooms, and heat exchangers. MODSIM plus the building component library is then called HVACSIM. HVACSIM, as distributed, can simulate systems of up to 600 variables and 50 simultaneous differential equations. These 1 imits can be increased, with a concurrent increase in program size. Current limitations on the size of simulations are given in section 3.10, page 37. The procedure for changing these limitations is summarized in section 5.6, page 51.

The simulation configuration program, called HVACGEN, for HVAC simulation GENeration, is also specific to building systems because the names of the categories of variables in simulations (temperature, pressure, humidity, etc.) are used explicitly. HVACGEN allows the user to specify the component models to be included in the simulation. The component models available are listed and described in a file of information about the HVACSIM component library 
which may be expanded or modified. The configuration information for a simulation is stored in a file called a work file. HVACSIM accepts information from a file called the model definition file. A small program translates the information in the work file to the form of the model definition file. This program is named SLIMCON.

This manual contains five chapters in addition to this introduction and the references. Chapter two provides basic information about the structure of an HVACSIM simulation and describes the preliminary procedures that are required before the HVACGEN and HVACSIM programs can be used.

Chapter three explains the use of the HVACGEN program. It describes the commands and procedures to create new simulations and edit existing simulations whose descriptions are stored in work files. Also described is the creation of a model definition file from a work file.

Chapter four summarizes the procedure for running the HVACSIM simulation program using a model definition file and other input files.

Chapter five is for the person responsible for installing the HVACSIM ${ }^{+}$package on a particular computer, diagnosing problems encountered with the package, and making changes to the programs. Guidelines are given for some specific machines as to computer memory and disc requirements and specific customization requirements.

Chapter six is a very important part of this manual because it provides a number of examples of the complete procedures for developing and running simulations with the HVACSIM ${ }^{+}$package. The examples range in complexity from a simple system involving a pipe and a valve to a simulation of an air handling unit connected to a building zone. As well as providing instruction in the use of the HVACSIM ${ }^{+}$package, the examples provide benchmarks for determining if the programs are correctly installed on a particular computer. The results generated on a computer where HVACSIM has been newly installed can be compared with the results in the examples, and if the results are within the machine precision of the target computer, it can be assumed that the package is correctly installed. 


\section{DEVELOPMENT OF A SIMULATION}

Developing a simulation which can be run by the HVACSIM ${ }^{+}$package requires some preparation before the computer programs are run. The information that is to be entered into the computer needs to be organized and a diagram of the system to be simulated should be made. This chapter describes these procedures. In addition, this chapter provides basic definitions and information that is required for an understanding of the HVACSIM ${ }^{+}$programs.

\subsection{Structure of an HVACSIM Simulation}

The simulation of a building system using HVACSIM requires that the user be familiar with the equipment and connections between pieces of equipment for the system to be simulated. A schematic drawing of the system is a convenient aid to visualizing what parts make up the entire system. Simulation of this system requires that a model be conceptualized which approximates the characteristics of the real system. The model of the system must be subdivided into component parts much as a real system is divided into component pieces of equipment. The basic component of the system model is called the "unit." A system model will consist of a number of units connected together. Each unit may be thought of as a model of a corresponding piece of equipment such as a pump, fan, pipe, or heat exchanger.

Each unit in a simulation is designated as being of a certain "type." A type designation indicates that a general model of a specific component is to be used for a unit. A unit might be assigned, for example, to be of a type called "HOT WATER TO AIR HEATING COIL" which is designated as type 11. Within the program, the unit would then be represented by a subroutine called TYPEIl which contains the algebraic and differential equations describing the behavior of a heat exchanger to heat air with hot water.

To allow a unit which has been designated as being of a certain general type to represent a specific piece of equipment, each unit has parameters which must be given numerical values. Each type will have different numbers and names for parameters. For example, a unit of type 11, "HOT WATER TO AIR HEATING COIL" might have parameters to specify the air side and water side heat transfer coefficients, the air and water flow resistances, the volume of the coil, and the thermal mass of the coil. Two units in a simulation might be of the same type but have different parameters.

Just as real system components are grouped into sub-systems, units may be grouped into sub-systems called "blocks." Groups of blocks may in turn be grouped into "superblocks." A superblock would be used to model a relatively complex building subsystem such as the building shell or an entire air handling system. The criteria by which units are grouped into blocks and superblocks are discussed in section 2.5 .

The type of a unit determines what differential and algebraic equations are to be used to model the unit. The parameters determine what the coefficients of the equations will be. The variables in the equations are the simulation variables. The independent variables in the equations will be the inputs to 
the unit and the dependent variables will be the outputs. Each unit will have several variables, some of them input variables and some of them output variables. The number of variables and their names will depend on the type assigned to the unit.

Variables are identified in the simulation by an index number and by the category of the variable. Variable categories correspond to different physical quantities such as temperature, pressure, and flow rate. Table 2-1 lists the variable categories that are available in the HVACSIM program. All variables of a particular category in the simulation will be numbered from one upward. Several variables may have the same index number but be distinguished by category. For example, there may be a pressure 1 and a temperature 1.

Table 2-1 Categories of Variables

\begin{tabular}{|c|c|c|}
\hline 1 & PRES & or \\
\hline 2 & FLOW & or \\
\hline 3 & TEMP & or \\
\hline 4 & CTRL & or \\
\hline 5 & RVPS & or \\
\hline & ENGR & or \\
\hline & POWR & or \\
\hline & AHUM & or \\
\hline
\end{tabular}

Name
Fluid Pressure
Temperature
Control Signal
Rotational Speed
Energy
Power
Absolute Humidity

Units

-.-...-

kilopascals

kilograms/second

degrees Celcius

dimensionless

revolutions/second

kilojoules

kilowatts

kilograms water/kilogram dry air

Each type of component has a fixed number of inputs, outputs, and parameters, and each input and output is in one of the eight categories listed above. A component is included in a simulation by specifying its type number. Each of its inputs and outputs must then be identified by assigning it an index number.

The complete set of variables in the simulation may be referred to by the term "state vector." At any time during a simulation, the state vector completely describes the state of the system. When the simulation is started, all variables must have a starting or initial value. This value can be specified in one of two ways. One way is to use the initial values which must be entered when a simulation is created using HVACGEN. The initial values should be as close as possible to the state at which the simulation should start. Alternatively, the initial state can be defined by the final state of a previous run of the same simulation. In this case the initial state supplied to HVACGEN is simply ignored.

\subsection{Types Component Library}

After the system to be simulated has been decided upon, the decomposition of the system into parts that can be modeled by units is required. Before this can be done, the user must know what unit types are available. A 1ibrary of approximately 30 types is supplied with HVACSIM $^{+}$. These component models are 
summarized in the Appendix. A separate document [1] describes the available types in more detail, and also describes how to build a custom type and add it to the library.

HVACGEN recognizes and accept alphanumeric names for types, provided that they are entered exactly as shown in the Appendix to this document. However, it is generally quicker and easier to identify each type by number. The appendix gives the number and name of each type, and brief definitions of the inputs, outputs, and parameters. With this information, the user can model the components of the system to be simulated by using the unit types available. If an exact match for a component in the system to be simulated is not available, the user can either use another type as an approximation or build a custom type.

\subsection{The System Diagram}

Once the types to be used in a simulation have been identified, it is suggested that a system diagram be constructed. This is simply a schematic diagram of the system, broken down into units, blocks, and superblocks, with state variables labeled on the diagram. Chapter 6 contains such diagrams for each example simulation. These diagrams make it much easier to visualize the physical significance of each variable in the simulation.

It may also be helpful to prepare an "information flow diagram" such as the one in the first example of Chapter 6. In this diagram, units are shown as icons or boxes representing actual system components. Lines between the units show the connection of one unit to another. Each unit is given a type number. As the information for the simulation is entered into the computer, additional notes on this drawing will ease the process of constructing a complex simulation.

\subsection{Unit Interconnections}

The connection of units is accomplished by the proper selection of the index numbers for the input and output variables of the units. If two units are connected, some item of information is passed from one unit to the other. Often this means that some fluid, most commonly air or water, is flowing through one unit and into the other. Alternatively, a control signal or a flow of thermal energy could pass from one unit to another. To indicate a connection, a variable associated with the output from the first unit would have the same index number and category name as the variable associated with the input to the second unit.

A connection between two units may actually involve a number of variables since (for example) a fluid stream has properties of at least temperature, pressure, and flow rate. The connection of units is also complicated by the fact that certain variables which might be regarded intuitively as outputs to a unit are actually inputs. The outlet pressure for a fan, for example, might normally be regarded as an output, but the "FAN OR PUMP" type requires the outlet pressure to be an input to the unit. 
In general, no variable in a simulation should be an output of more than one unit. If two units produce the same variable as output, it usually means that the set of equations implied by the input/output connections is overdetermined. In such cases, either the two outputs should be given different indices, even though they represent the same physical quantity, or one of the output variables should be given an index of zero, which effectively removes the output from the simulation. Otherwise problems may occur when the type equations are solved by HVACSIM. The program SLIMCON issues a warning message if two or more outputs in a simulation have been given the same index number in the same category.

All variable indices in each category can be pre-selected and noted on the system diagram after study of the types library. The notation can be a letter representing the variable category followed by the index number, and can be placed on the diagram between two units that are connected. An alternative is to note the indices on the diagram as the information is being entered into the computer with the HVACGEN program. It may also be helpful to make copies of the pages in the appendix and use these as worksheets for recording unit numbers, input and output index numbers, and parameter values.

\subsection{Grouping Units into Blocks and Superblocks}

In addition to selecting types and connecting units together, decisions are required for the grouping of units into blocks and the grouping of blocks into superblocks. There are no highly specific rules for the division of superblocks and blocks. The decisions involved must be based on knowlddge of how the real system behaves and an understanding of how blocks and superblocks are handled within the simulation program. The example chapter of this document provides some working examples of grouping of units into blocks. The following paragraphs will briefly explain the concepts of blocks and superblocks.

The general reason why a simulation is divided into blocks and superblocks is to reduce the time required to solve large sets of equations simultaneously. Each state variable which is both an input and an output can be thought of as representing an equation. If a simulation contains forty such variables, there are forty equations in need of simultaneous solution. The time required to solve a set of equations is roughly proportional to the square of the number of equations. Thus a set of forty equations takes at least twice as long to solve as two sets of twenty equations. The division of a simulation into blocks and superblocks is a way of partitioning the matrix of equations into a larger number of smaller sets of equations.

The set of input/output connections within a block define a set of "block equations." All equations for the units in a block are solved simultaneously. A block can then be thought of as equivalent to an unusually large unit, having input/output connections coupling it to other blocks in the simulation. The set of input/output connections between the blocks in a superblock define a set of "superblock equations." All superblock equations are also solved simultaneously, but in a manner involving preliminary solution of the block equations. 
At the highest level, the equations defined by input/output connections between superblocks are not solved simultaneously. Rather, they are solved sequentially, superblock by superblock, assuming that superblocks are essentially independent of each other. This allows superblocks to run with different time steps. It also implies that superblocks must represent subsystems that are loosely coupled. For example, an air handling unit and a zone are of ten only coupled through the air entering the zone from the air handling unit, and the response time of the zone is often much longer than the response time of the air handler. Even so, passing a flow stream or a control signal from one superblock to another inherently involves a tradeoff between speed and accuracy. Two special-purpose components (types 16 and 19) have been developed to alleviate the problems of passing information between weakly coupled superblocks.

As a rule of thumb, it is advantageous (though not essential, and not always possible) to keep tightly coupled sub-systems such as local closed loop control systems or closed loop flow streams within a single block. Blocks should be thought of as discrete components, differing from units only in size and complexity. In simulations of moderate size, it may be desirable to use only a single superblock, thus avoiding the assumption that subsystems in different superblocks are nearly independent. In large simulations involving subsystems with widely differing response times, the division into superblocks should separate fast systems from slow systems, to take advantage of the independence of superblock time steps.

Other constraints on the division into blocks and superblocks are the maximum limits of 20 units per block, 30 simultaneous equations per block, 10 blocks per superblock, 20 simultaneous equations per superblock, and 20 differential equations per superblock. These 1 imits are not fundamental, and can be increased if necessary (see section 5.6).

It is recommended that once the division of the simulation into blocks and superblocks has been chosen, the boundaries of blocks and superblocks should be shown on the system diagram. The visualization of blocks and superblocks on the diagram is also helpful since there should be a minimum of connections between units which pass though superblock or block boundaries. Once the diagram has been completed with unit, block, and superblock designations it can serve as a useful guide when entering the simulation information into the computer.

\subsection{Boundary Conditions}

In the context of HVACSIM, a boundary condition is defined as a state variable which is an input to at least one unit in a simulation but is not an output of any unit. Boundary conditions are divided into two categories: constant or time-independent boundary conditions, and time-dependent boundary variables. The latter are sometimes referred to simply as boundary variables.

A simulation description provides a model of a real system. Time-dependent boundary variables represent the environment to which the system responds. In 
a building system the environment might include external driving forces such as outdoor temperature and solar radiation, internal driving forces such as building occupancy and equipment utilization, and controlling forces such as scheduled equipment setpoint changes.

The HVACGEN program allows any variables in the simulation to be designated time-dependent boundary variables. Note that only boundary conditions should be so designated. The program SLIMCON issues a non-fatal error message if any simultaneously solved variables are declared as boundary variables.

The simulation program expects to find time-varying values for each of the specified boundary variables in a file. The structure of the boundary condition file is described in chapter 4. Constant boundary conditions, defined as boundary conditions which have not been declared time dependent, simply retain their initial values throughout a simulation.

\subsection{Reported Variabies}

Running a simulation produces files containing a record of the values of certain simulation variables at certain time intervals. The HVACSIM ${ }^{+}$programs allow any variable in the simulation to be designated as a reported variable. The time interval at which reported variable values are recorded in the output file can also be chosen. Different reporting intervals and reported variables may be used for each superblock. For example, a building zone superblock might have information reported at ten minute intervals (of simulated time) while an air handling unit might have its crucial variables reported every minute.

\subsection{Work Files and Model Definition File}

The information describing a simulation is stored in a computer file called a work file. There are three types of workfiles. A simulation work file contains all of the information required to describe a complete simulation. A second type of work file describes only the information for a block of units. Finally, the least complex work file is a unit work file which contains information on a single unit. The reason that block and unit work files are allowed is that it is possible using the HVACGEN program to insert new blocks and units into a simulation. This is done by taking the information in a block or unit work file and merging it into the information in a simulation work file to create a larger simulation.

The use of previously created blocks and units allows a large simulation to be developed from pieces which might have been previously used and tested in another simulation. Less effort is required to define the details of the blocks in a simulation. In addition it is possible to build up a large number of standarized units and blocks with the characteristics of specific real pieces of equipment from specific manufacturers. The parameters for the equipment need only be entered once, and then the user can create simulations in terms of these pieces of equipment rather than at a very basic level. 
The HVACSIM simulation program cannot use the simulation work file directly to run a simulation. The HVACSIM program reads the simulation description from a file called the "model definition file." This file contains preprocessed information derived from the simulation work file. To convert a simulation work file to a model definition file, a separate utility program called "SLIMCON" is used. This conversion need not be done during the model development process. When the simulation is ready to be run the model definition file can be created.

\subsection{Developing Complex Simulations}

A complex simulation can be built using the HVACGEN program. It is possible to insert blocks and units into a simulation, to delete blocks and units from a simulation, and to replace units with units of a different type. It is also possible to save blocks from a simulation for later insertion into the same simulation or other simulations. With the current version of HVACGEN, however, it is not possible to insert a superblock into a simulation or delete a superblock from a simulation. Therefore a special technique is required to build multi-superblock simulations from blocks and units previously created or saved.

If a simulation is to be built with one superblock, the first step is to create a framework for the final simulation by creating a simulation which has a single superblock with one block and one unit in the block. This is the minimum simulation that can be built with one superblock. The insert block and insert unit functions of HVACGEN can then be used to build up the simulation. If an additional superblock is to be added to this simulation, it cannot be done with the current version of HVACGEN.

If a multi-superblock simulation is to be built, the number of superblocks must be known before the framework simulation can be created. It is better to have an extra superblock which is not needed and which contains a unit not connected to any other unit, to allow for future expansion, than to be unable to add another superblock to a simulation.

When units or blocks are inserted into or deleted from an existing simulation or framework simulation, some potential confusion may arise with regard to the connections of the new units to the existing units and the numbering of units and blocks. Units and blocks in a simulation are assigned numbers as they are added to a simulation. These numbers are not fixed, and may change if a unit or block is deleted from the simulation. For example, consider the case where there are five blocks in a superblock, numbered from one to five. If block two is deleted, block one will still be named block one, but block three will become block two, block four will become block three and block five will become block four. After the deletion there will be no block five. As blocks or units are added, the numbers they are given will be the next number in series in the simulation and will have no correspondence to the block or superblock into which they are placed.

If a unit is inserted into a simulation, the input and output variable indices that were stored in the unit work file will not correspond to any indices 
currently in the simulation. The indices should be immediately edited to connect the unit to other units in the simulation. The initial values should also be entered for any new variables created by the insertion. A unit will typically be inserted between two existing units which are connected to each other. In this case, the variable indices of at least one of the adjoining units must also be edited to properly connect the new unit.

When a block is inserted into a simulation, the input and output variable indices are probably incorrect. Although the units within the block will be properly connected to each other, the variable index numbers within the block work file may also exist somewhere else in the simulation. HVACGEN has the capability to partially alleviate this problem by optionally renumbering the index numbers within a block being inserted into a simulation. This option can greatly reduce the amount of variable index editing required.

\subsection{Summary of Model Development Procedure}

A summary of the suggested steps in developing and running a simulation is given in the table below. If mistakes are made, some steps will have to be repeated before moving on to the next step.

1. Determine system and components

2. Determine types available and select types

3. Diagram types and connections

4. Select parameters for types

5. Group units into blocks

6. Group blocks into superblocks

7. Select Boundary variables

8. Select Reported variables

9. Determine initial values

10. Run HVACGEN to create simulation work file

11. Run SLIMCON to create model definition file

12. Set up boundary value file

13. Run HVACSIM

14. Interpret results

The order of the first ten steps may be varied to some extent. For example, in some some situations it might be more convenient to create a simulation with arbitrary parameter values and subsequently edit the simulation to enter realistic parameter values. 


\section{HVACGEN - THE HVACSIM MODEL CONFIGURATION PROGRAM}

This chapter provides the reference material for operating the HVACGEN program used to configure simulations which are to be run using the HVACSIM simulation program. Sections 3.1 through 3.4 discuss general information about the program and its use. The sections following 3.4 describe the commands available when running HVACGEN.

\subsection{Basic Commands: CREATE, VIEW, and EDIT}

There are three basic types of operations that the HVACGEN program performs. All operations are oriented around the concept of the work file. The work file contains all information describing a complete simulation, a simulation block, or a simulation unit. The basic operations are used to modify the information in these work files.

The first basic operation is CREATE. This operation is used to produce a new work file with information entered from the keyboard. No previous work file is required to use the create operation.

The second basic operation is VIEW. For this operation a work file must have been previously created. VIEW allows the information in the work file to be examined in a simple manner and displayed on the computer terminal. The original work file is left unaffected by the VIEW operation.

The third basic operation is EDIT. This is a powerful operation which allows the information contained in a work file to be changed, added to, or reduced. A work file must have been previously created. The information in the existing work file is manipulated using the computer terminal and the edited information is written into a new file or back to the original file.

\subsection{Control of the Program}

In any computer program used for interactive operations with the program user, there are a number of ways for the user to control the program using a keyboard. At one extreme is the program which simply presents a prompt and expects the user to enter commands consisting of one or more characters to direct the program. This is a command driven system. At the other extreme is the program which gives the user a "menu" of choices at every decision point. This is a menu-driven system.

Another distinction between program control methods is how many of the total set of commands or menu choices available in the entire program can be used at any time. Usually a menu-driven system has a hierarchy of menus which allows only a small subset of the total menu items from all menus in the program to be used. A choice on the first menu (or main menu) produces a secondary menu. A choice on the secondary menu produces a tertiary menu and this process continues depending on the complexity of the program. Once on the tertiary menu, however, a choice from the main menu cannot be entered. An extreme command driven system may allow any of the program commands to be entered at 
any time. Command driven systems may also have a hierarchy in which only certain sub-commands may be entered after a particular command has been entered.

The HVACGEN program is a combination of a menu and command driven system, and there is a hierarchy to the commands. However, there are also some commands which may be entered at any time. The HVACGEN program has a main menu which displays the main program commands. These may be entered as shown on the menu and, like a menu driven system, a secondary menu will be displayed asking for a subcommand. However, for the user who is familiar with the program, the levels of menu after the main menu may be bypassed by typing all of the subcommands for a main menu command on the same line. For example, a user might enter "EDIT SIMULATION HVAC3 STRUCTURE." The program would go directly to the portion of the program which allowed editing the structure of a simulation with the name HVAC3. If only "EDIT" were entered, other menus would prompt for what type of work file to edit, the name of the workfile, and what part of the work file to edit.

The lower levels of the program hierarchy are more of a completely menu driven system. A number of choices are displayed on a menu and only choices from that menu may be selected. Choices from the next menu may not be stacked onto the first choice. The transition from command/menu system to pure menu system is indicated by the change of menu choice entry from alphanumeric commands to menu-choice index numbers.

As mentioned, there are some commands which may be entered at any time, even if the program is asking for numerical input. This type of command, which may be referred to as "transparent," is used for purposes such as aborting the current operation, getting help, examining information on available types, or editing the information for a unit which has been already entered during the creation of a simulation or block work file.

The ABORT command may be typed at any time. This command causes an unconditional return to the main menu. If a work file is being edited, all changes will be discarded and the file will be left in its original state. If a file is being created, all information entered will be lost. To avoid accidental loss of data, the program prompts for verification of the ABORT command.

The HELP command may also be typed at any time, except when the program is expecting a filename entry. General information on the program will be displayed. Following the help information, the program returns to where it was when the help command was entered.

EDIT and VIEW commands may be used to edit and view units in a simulation or block work file which is currently being created. Any unit for which inputs, outputs, and parameters have been previously entered can be edited or viewed without terminating the process of creating the simulation or block. Table 31 shows the "transparent" commands and when they may be used.

\subsection{Command/Menu Syntax}

When commands in the upper levels of the program are shown on menus with the 
Table 3-1 Transparent Commands

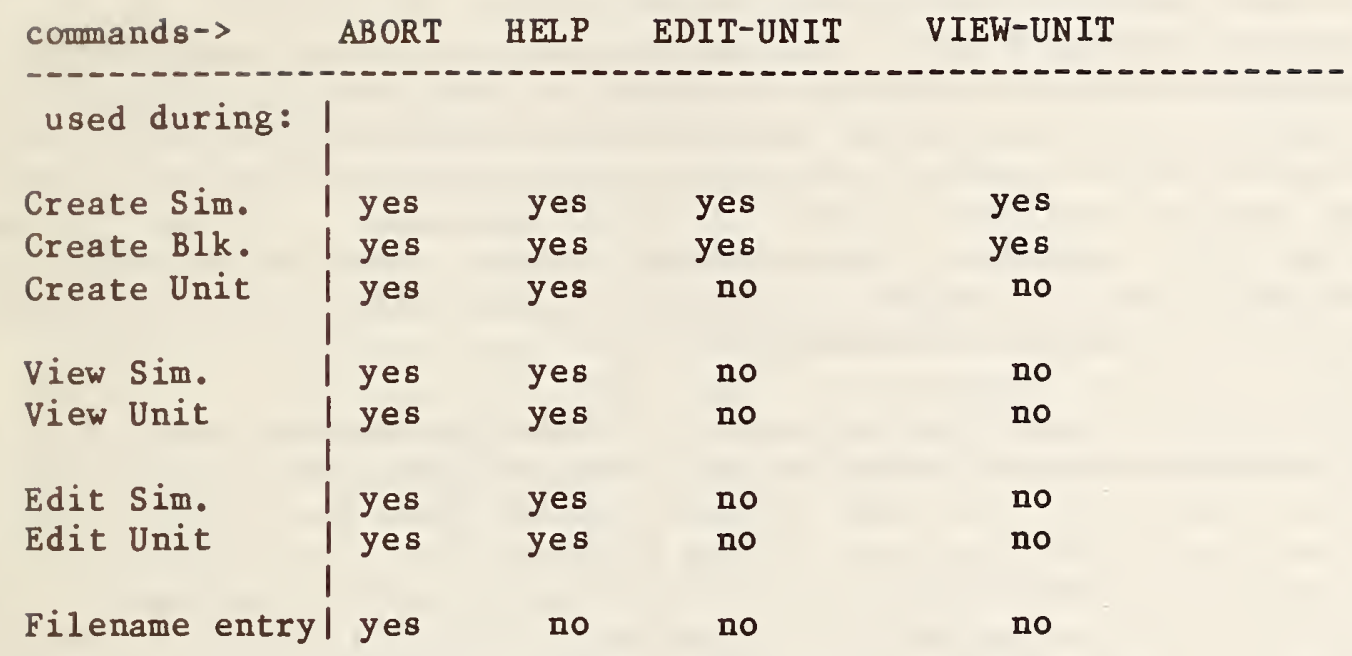

first two letters capitalized, the command may be entered by shortening the command to the capitalized letters. For example, to enter the CReate command, the user can enter "CREATE". "CRE", or "CR". This applies to the stacking of commands on one line. Therefore "EDIT SIMULATION HVAC3 STRUCTURE" may be entered as "ED SI HVAC3 ST".

In some cases the program will display a value and ask if it is acceptable. The proper answer is yes or no. Yes can be abbreviated to the letter $Y$ or $y$. In most cases yes can also be entered by a simple carriage return. No can be entered using the letter $N$ or $n$. Either upper case or lower case responses may be used.

When a number is requested which is a real number (such as a unit parameter), it is not necessary to enter a decimal point for whole numbers. The program does not allow numbers which are unreasonable to be entered. An error message is given if the number is out of range, and the user is asked to re-enter the number.

\subsection{Basic Program Operations}

There are two sequences of basic program steps which are so commonly encountered that they are described in one place and referred to by a shortened name. These are the "save sequence," used to save information in files, and the "edit sequence," used to change a list of previously assigned values.

\subsubsection{Save Sequence}

The program operations are performed on the information in work files which are for blocks, units, or simulations. The work files are referred to while in the program by a filename. This filename can have a maximum of eight 
characters consisting of only numbers and letters. The actual operating system filename used for the work file may not be exactly the same as the eight character name used while running the program, but incorporates the eight character name in the operating system filename along with a way to identify the file as a unit, block, or simulation work file. To refer to this file from the program, however, only the original 8 character file name as entered here need be used. For example, on many computers, a system file name consists of an eight character filename with a three character extension such as "FILENAME.EXT". For a simulation work file saved under the name "BLDG25", the system filename would be "BLDG25.SIM" .

The "save sequence" is used by the program to save information in work files. If a change is made to any simulation data, the program attempts to save the changes in the work file. The save sequence begins when the program asks for a filename for saving information. If any filename has been previously used (to read in work file information) the filename last used is displayed and the program asks if it is acceptable to save under this file name. A carriage return or "Yes" answer will then save the changed information under the file name last used. This is convenient when editing data in a work file and saving the changed data in the same file. If a different filename should be used, a "No" answer should be given to the question. The program then prompts for a new filename and saves the simulation information under that name. If no filename has been previously used (such as in the case where the program is started and one of the create commands is used and a filename is needed to save the entered information) the program simply requests a filename. The "save sequence" is encountered often and provides a maximum of safety in avoiding loss of editing changes.

If an illegal filename is entered during the save sequence, the program prints an error message and ask for the filename again. If a filename is entered that is longer than eight characters, the name is truncated to eight. The ABORT command can be used when a filename is requested, which prevents the saving of any information in a file. For this reason filenames starting with the characters " $\mathrm{AB}$ " cannot be used.

Depending on the exact implementation of the HVACGEN program, backup files may or may not be available. If backup files are used, then whenever a work file is edited, a copy of the file is saved as it was before changes were made. If additional changes are made, the original backup is replaced with the next most recent version of the work file. The backup file is created at the time that the filename to edit is entered. If the editing is aborted, the backup file will then contain a copy of the work file as it was when the most recent EDIT command was given.

\subsubsection{Edit Sequence}

The "edit sequence" consists of displaying a list of values one at a time, and requiring the user either to approve or to reject and modify each value before the next value is displayed. The edit sequence is encountered only in the EDIT section of HVACGEN, and begins when certain categories of information have been selected for editing. For example, a request to edit the parameter 
values of a particular unit invokes the edit sequence.

The program begins the sequence by displaying the first value in the selected category and asking if the displayed value is correct. If the answer is yes or carriage return, the value is left unchanged and the program displays the next value in the category, again asking if the current value is correct.

If the current value is not acceptable and the answer given is no, the program prompts for a new value of the item. After a new value is entered, the program displays the new value and again asks if this value is correct. This procedure is repeated as many times an necessary until all items in the category have been approved. Note that once approved, a value cannot be changed again until the entire list of values in the category has been completed.

\subsection{The Main Menu}

When the HVACGEN program is started, a menu of the basic commands is presented on the computer console. This menu allows a choice of the commands CREATE, EDIT, VIEW, and HELP plus the command END which ends the program. Also shown is the version number of the program and the date last changed. The create, edit, or view commands may be entered in the single word form as shown on the menu, and the program then asks for additional information as necessary. Alternatively, the commands may be entered in the multiple word forms shown as the titles of the subsections under the CREATE, EDIT, and VIEW command descriptions on the following pages. Descriptions of all commands will be found in the remaining sections of this chapter. Figure $3-1$ is a diagram showing the major commands, subcommands, and the menus which are presented.

\subsection{The Create Command}

The command CREATE is entered to begin creating a new simulation, block, or unit. This command is used after a simulation or part of a simulation has been conceptualized and a diagram of the system to be simulated has been produced (as described in chapter 2). Entering CREATE takes the user to a portion of the program where information is entered in a highly prompted manner to produce an initial specification for a simulation, block, or unit. The CREATE command can be entered alone or along with the type of construction to be created, either UNit, BLock, or SImulation on the same line (e.g. CReate UNit). If the CREATE command is used alone, a menu prompts for the choice of what to create.

While in the CREATE section of the program, the user may use the EDIT UNIT and the VIEW UNIT commands at any time when not actually entering information for a unit that is to be viewed or edited. Also while in CREATE, the HELP command may be used at any time.

At any time while in create, the powerful command ABORT may be used to end the current creation of a unit, block, or simulation. However, if ABORT is used before the entry of a file name to save the created information, all information will be lost. The ABORT command returns the program to the main menu. 


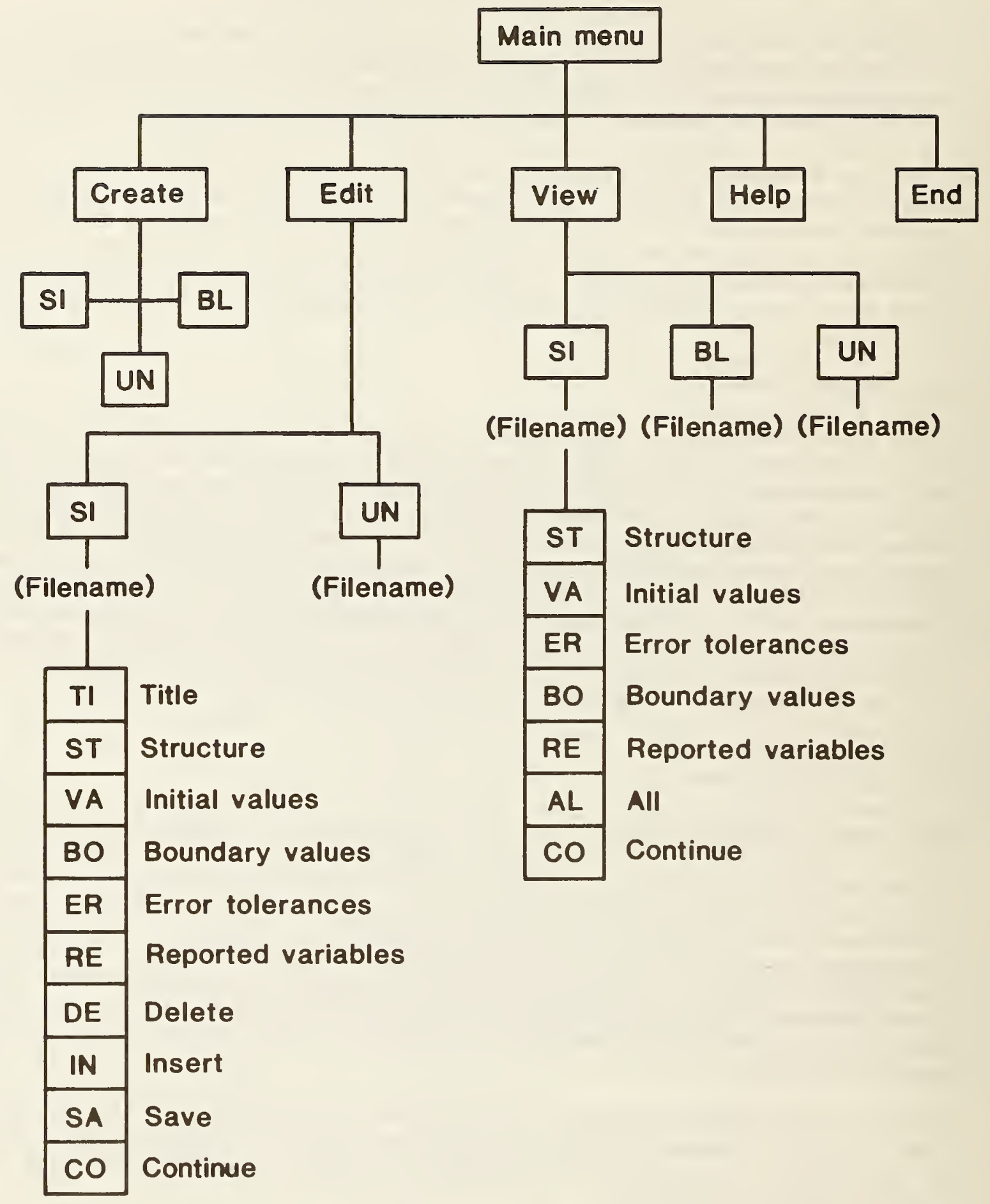

Figure 3-1. Schematic diagram of major commands of HVACGEN 


\subsubsection{CReate UNit}

If UNIT creation is selected, the number designating the type of the unit to be created must next be entered. If the number of the type is not known, a list of all types in the library can be obtained on the screen by entering the command TYPES at the prompt for the unit number.

When a valid type descriptor has been entered, the program prompts for the index numbers of the input variables. The index number may be any integer between 0 and 100. If a number outside this range is entered the program gives an error message and asks for the index again. Within this range, and at this point in creation, there is no restriction on the index number used. In prompting for index numbers, the program identifies each variable by category (see table 2-1) and description.

After all input variable indices have been entered, the program prompts for output variable indices. Note that all outputs in a simulation should be distinct. For example, if two units calculate flow rates which must be identical due to conservation of mass, the two outputs should not be given the same index number. One of the outputs may be given an index of zero $(0)$, which causes the value of that output to be ignored, or different indices may be used for the two physically identical variables. The program SLIMCON (but not HVACGEN) issues a warning message if two or more output variables in the same category share the same nonzero index number.

As each index for a variable is entered into the program, the user should ensure that the index number is recorded on the diagram being used as a guide to create the simulation. The index numbers define the structure of the system model, in that they determine the flow of information from one component to another.

After all input and output indices have been entered, the program prompts for type parameters. These numbers indicate physical properties or coefficients for determining the behavior of a particular type. Each type has a fixed set of parameters. The prompt for the type parameters includes the alphanumeric description of the parameter and the engineering units in which the parameter is to be entered. Parameters need not be entered with a decimal point if the number does not have a fractional part.

After all parameters have been entered, the program prompts for a file name and executes a "save sequence. No default filename will be presented. The unit description is stored in a unit work file and the program returns to the main menu. At this point, there is no difference between having entered the program for the first time and having just created a unit. There is no intermediate storage for the unit description. Any editing or viewing of this unit description must be done using the file just saved.

\subsubsection{CReate BLock}

When a block is created, it is assigned a block number by the program. This block number is reported after the create block command is entered, but has 
little meaning. Block numbers are only significant when a simulation with multiple blocks is created or edited.

Creating a block consists of creating a series of units. The creation of each unit in a block is the same as creating a single unit (SEE CREATE UNIT). The user is prompted for the type of the first unit in the block, then for the indices of the input variables, the indices of the output variables, and the values of the type parameters. If an incorrect or unknown type designation is entered, the program repeats the prompt for the name or number of the type. A list of the available types can be obtained by entering the command TYPES.

After all information for the first unit has been entered, a message reports the maximum number of units allowed per block and the number of units that can still be entered in the current block. The user is then asked if he would like to continue entering units. The answer to this question determines how many units will be placed in the block. If a single unit is to be placed in the block, the question is answered with a no. If more than one unit is to be included in the block, the question is answered yes. After the information for the last unit in the block is entered, the question is answered no. The answer yes or no can be "YES", "yes", "Y", "y", "NO", " nO", "N", or "n".

In response to a yes answer, the program prompts for type number or name, variable indices, and parameter values for the next unit in the block. After each unit is entered, the number of available slots for entry of units in the block is given and the question on continuation of unit entry is repeated. If the maximum number of units has been entered, the program automatically ends the entering of units.

If the question on continuation is answered no, or if the maximum number of units has been entered, the block is then finished and the program goes through a "save sequence." The program then returns to the main menu, ready to allow the selection of a new function.

If a mistake is made while entering information for a unit (except for the type number, see below) the user can correct the error after the rest of the information for this unit has been entered. When the program asks if the user wishes to continue entering units, there is an EDIT UNIT command which allows editing of the information for units which have already been entered. The EDIT UNIT command can be used during the process of creating a block. The use of this command is described below, in the section on the EDIT command (section 3.8 .1 ).

If the wrong type identification is entered, there is no way to immediately correct this mistake. The user may either use the ABort command, which discards all information in the block and returns control to the main menu, or he may supply arbitrary input, output, and parameter information (e.g. zeros) to get to the next prompt for information on a new unit, and then proceed with the correct information.

The current version of HVACGEN has EDIT commands for modifying unit and simulation work files, but not block work files. After a block work file has been created, a rather indirect approach is required to correct errors or other- 
wise modify the information it contains. The EDIT SIMULATION commands can be used to insert the block into an existing simulation, edit the information, and wite the modified block to a block work file again. These procedures are explained in section 3.8 .2 .

\subsubsection{CReate SImulation}

Since a simulation consists of superblocks which are made up of blocks which in turn are made up of units, the program must keep an accounting of the current superblock number, block number, and unit number. When the CREATE SIMULATION section of the program is invoked, the program assigns the number 1 to the first superblock to be created, the first block in this superblock, and the first unit in this block. As each additional unit, block, or superblock is added, the appropriate number is incremented.

The creation of a simulation requires the entry of two types of information. First is the information on the units, including their assignment to particular blocks and superblocks. This category constitutes the structure of the simulation. Second, simulation creation requires the entry of information which controls the operation of the simulation. This includes designation of boundary variables, initial conditions, variables to be reported, reporting intervals, and error tolerances.

\subsection{3(1) structure information}

The entry of information on the units is exactly the same as for the CREATE UNIT and CREATE BLOCK commands. The program prompts for unit type, input and output variable indices, and type parameters. After the entry of this information, the program displays the number of additional units that may be placed in this block and asks whether additional units are to be added to the current block. If the answer is yes, the program prompts for the information on the next unit in the current block. If the answer is no, the current block is closed. The program then lists the the number of additional blocks that can be placed in the current superblock and asks whether additional blocks are to be added to this superblock. If this question is answered with a yes, the program increments the block number and asks for information on the units to be placed in the new block.

When no additional blocks are requested, the current superblock is closed. The program then displays the number of additional superblocks that may be placed in a simulation and ask if additional superblocks are to be added. If an affirmative answer is entered, the superblock and block counters are incremented and information on the first unit in the first block of the next superblock is requested. Note that units and blocks are numbered for the entire simulation and do not start at one in each new superblock. Each time a new superblock or block is begun, the user should note the assigned block number on the planning diagram.

If a mistake is made while entering information for a unit (except for the type number, see below) and the carrage return key has already been pressed, the user should not attempt to correct the error until the rest of the infor- 
mation for this unit has been entered. When the program asks if the user wishes to continue entering units, there is an EDIT UNIT command which allows editing of the information for units which have already been entered. The EDIT UNIT command can be used during the process of creating a simulation. The use of this command is described below, in the section on the EDIT command (section 3.8.1). If, however, the wrong type identification has been entered, there is no way to immediately correct this mistake. Although the EDIT command allows deletion or replacement of units (which is the only recourse if the type number is wrong), this cannot be done while executing the CREATE SIMULATION command. If an incorrect type number is entered, the user should then supply arbitrary input, output, and parameter information (e.g. zeros) to get to the next prompt for information on a new unit, and then proceed with the correct information for the unit. After the creation of the simulation is complete, the erroneous unit can be easily deleted or replaced using EDIT commands.

To end the entry of unit information for the simulation when all units have been described, all questions about continuing to enter units, blocks, or superblocks should be answered with a no. After the program receives the information that no more superblocks are to be entered, it asks for the additional information required run the simulation.

\subsection{3(2) simulation control information}

After all the unit description information is entered, the title of the simulation is requested. The title can be up to 80 characters in length and may contain any alphanumeric characters. The title is used to give a brief description of the system being simulated.

After the title of the simulation is entered, a decision must be made about what values to use for the simulation program error tolerances. There are four different error tolerances, and a default value is available for each one. The default tolerances are somewhat arbitrary, but generally produce satisfactory results. The significance and effect of these error tolerances is described in reference [1]. Briefly, RTOLX and ATOLX are relative and absolute error tolerances, used primarily to decide when a variable has reached steady state and may be "frozen," that is, removed from the set of equations being solved, and when a frozen variable has begun to change significantly and should be "unfrozen," or reinserted into the equation set. ATOLX is often much less than RTOLX; its purpose is to prevent division by zero when a variable has a value of zero and the relative error is undefined. XTOL is an error tolerance used exclusively by the simultaneous equation solving portion of the simulation program. The recommended value of XTOL is the square root of the machine precision, which varies from computer to computer. TTIME is defined as the time interval over which the integral of a differential equation must be within error tolerances. Increasing TTIME results in a larger number of shorter time steps during the course of a simulation. Limited experience suggests that values between 1 and 100 are reasonable, with 5 or 10 being fairly typical.

In most cases the user may simply answer "yes" and use the default values, but experimentation is encouraged. 
The program then proceeds to calculate, from the previously entered information, the total number of variables and the number of variables in each category such as pressure, flow, temperature, and so on. A report of the totals is written to the terminal. In order to run a simulation, the program must be given initial values for all of these variables to use as a starting point. The program prompts for initial values to be entered one at a time. The prompt includes the category of the variable (such as pressure) and the variable index. This is the same index that was entered during the unit specifications, which the user should have written down on the model specification diagram.

The prompt for initial yalues begins with variable index one and proceed in increments of one up to the maximum number of variables in that category. It is quite possible that variable index one or any other index might not have been used when entering the unit specifications. The initial values are stored in a list or array of numbers which must have space for all variable values up to the maximum index for each category. This list is called the "state vector." The state vector must have all values filled in even though a particular index was not used. If an index is not used the number entered for the initial value is unimportant. The use of zero is a good practice, since carriage returns are treated as zeros. If a variable index was used in entering the unit specifications, the number entered for the initial value should be significant.

When all initial values for a particular category have been entered, the program begins prompting for the initial values of variables in the next category.

After all initial values have been entered, the program prompts for the designation of time dependent boundary variables. As discussed in section 2.6, any unit input variables which are not outputs of any unit in a simulation are considered boundary conditions. If not designated as time dependent, these variables will retain their initial values throughout a simulation. Typical examples of time dependent boundary variables are setpoints, outside air temperature and humidity, and fan speeds. Any of the variables in a simulation may be declared time dependent boundary variables. The HVACSIM simulation program expects to find values for the time dependent boundary variables, and the corresponding values of time, in an external file when the simulation is run.

For each category of variable, HVACGEN prompts for the index numbers of any variables in that category which should be boundary variables. If there are no boundary variables in a particular category, or if all boundary variables in a category have been entered, a carriage return causes the program to move on to the next variable category.

When a simulation is run, the output consists of the values of certain of the simulation variables at each time step and at some fixed time interval different from the time step. The HVACSIM program allows any of the variables to be reported. Since superblocks may have different time steps, it is possible to report variables in different superblocks at different frequencies. For ex- 
ample, variables in one superblock may be reported every five seconds while variables in another superblock may be reported only every sixty seconds. After all boundary variables have been designated, the program prompts for reported variables. These are grouped by superblock.

The program begins prompting for reported variables by requesting a reporting interval for the variables in superblock one. All variables in superblock one designated as reported variables will be reported at this interval. For each category of variable, the program asks for indices of variables to be reported. The entry of values is identical to the entry of boundary variable designations. If no variables in a category are to be reported, or all reported variables in a category have been entered, a carriage return causes the program to move on to the next category of variables for that superblock.

After all reported variables for a superblock have been designated, the program prompts for values of the "variable freezing option" and the "input scan option." Possible values for the variable freezing option are 0,1 , or 2. Possible values for the variable input scan option are 0 or 1 . The significance of these options is discussed in reference [1], pages 5, 6, and 8 . Briefly, the freezing option influences the strength of coupling among the blocks within a superblock, and has no effect on simulations consisting of a single block. The input scan option influences the strength of coupling between superblocks, and has no effect on simulations consisting of a single superblock. In both cases, the choice involves a tradeoff between speed and accuracy. The zero options reduce the amount of computation required, at the cost of reduced accuracy. The recommended procedure is to begin with the high values, experimenting with the low options only after a simulation has been run successfully.

After all information for the first superblock has been entered and if there is more than one superblock in the simulation, the program requests a reporting interval for the next superblock in the simulation. This reporting interval may be different from the reporting interval of the other superblocks. As with the first superblock, the reported variables are designated by entering the variable indices for each category of variable. A variable freezing option and an input scan option are also requested for each superblock.

Entry of all reported variables and options completes the specifications for CREATing a SIMULATION. At this point the program prompts for a file name under which to store the simulation. This name, like the block and unit filenames, is an eight character name which uniquely identifies the simulation work file. After the file name is entered and the workfile is stored, the program returns to the main menu.

\subsection{The View Command}

The view command allows a unit, block, or simulation work file to be examined for purposes of documentation or review. The command may be suffixed with UNit, BLock, or SImulation to select the type of work file to be examined. If the command suffix is not entered, the program prompts for the type of work file to be viewed. 
When the view command is invoked from the main menu, the item to be viewed must be an existing unit, block, or simulation work file, and therefore a filename is required before the file can be viewed. The file name may be entered along with the VIEW command and the work file type suffix. If this is not done, the program prompts for the filename. Each of the following section headings represents the full form a view command as it may be entered when prompting is not desired.

Under certain conditions, the VIew UNit command can be used without ever entering a filename. This use of the command is discussed in the next section.

\subsubsection{VIew UNit <filename>}

The VIew UNit command displays the unit number, the type of the unit by name and number, the category, index number, and definition of each input and output variable, and a list of all parameters with names and values. The program pauses after one page of information has been displayed. A carriage return may be entered to continue the display.

When entered from the main menu, the VIEW UNIT command is used to examine the contents of a unit work file. After all of the information on the unit has been shown, the program returns to the main menu.

The VIEW UNIT command can also be entered at any time during a CREATE BLOCK or CREATE SIMULATION operation. In this case the command is used to review units in the work file currently being created, and so no filename is required. Using this command, any unit (for which all the inputs, outputs, and parameters have already been entered) can be viewed at any time without terminating the CREATE BLOCK or CREATE SIMULATION operation. After VIEW UNIT is invoked, the program re-prompts for the information that was requested when the view command was entered.

\subsubsection{VIew BLock <filename>}

The VIEW BLOCK command displays information about the units within a block work file. If the filename is not specified with the VIEW BLOCK command, it is requested by the program. The program lists the units in the block with their type numbers and names. It then allows any of the units within the block to be viewed in greater detail, displaying the category, index number, and definition of all inputs and outputs, and the values and definitions of all parameters. This display is exactly the same as the one produced by the VIEW UNIT command. After the information is displayed, the program prompts for another unit to be viewed. A carriage return may be entered when a unit number is requested in order to return to the main menu.

To get detailed displays of all of the units within a block, the user must type in the unit numbers for each of the units in the block one at a time. This is currently the best way to document the contents of a particular block 
work file.

The VIEW BLOCK command is file oriented and cannot be invoked from any point except the main menu.

\subsubsection{VIew SImulation <filename>}

The VIEW SIMULATION command displays information contained in a simulation work file. The name of the work file to be viewed may be typed on the same line as the view simulation command. If the filename is not specified, the program prompts for the filename. After the program receives the view simulation command, a menu is presented which allows any part of the simulation information to be displayed. The choices for viewing simulation information are as follows: STRUCTURE, which gives information on the units in the simulation and how they are arranged in the blocks and superblocks; variable initial values: error tolerances; boundary variables; reported variables; ALL, which is intended for simulation documentation and provides a complete simulation description; or CONTINUE, which returns the program to the main menu. The view simulation sub-command may also be typed on the line with the view simulation command as in the sub-section headings below.

\subsection{3(1) VIew SImulation 〈filename> ALL}

In response to this command, the information available from the other view commands is sent in sequence to the terminal without any pauses for each page. This results in a complete description of the simulation being output with a single command. This command is intended to allow this description to be saved in a file or printed. Specific computers will have different methods of redirecting or capturing console output to a printer or a disc file.

\subsection{3(2) VIew SImulation <filename> STructure}

This produces on the terminal a listing of the superblocks by number, with the blocks in each superblock shown under the superblock heading and the units in each block shown under the block headings. The program prompts for a unit number to allow the details of a particular unit to be examined. After the unit information is viewed, the program asks for another unit number for viewing. A carriage return can be used to return to the VIEW SIMULATION menu.

\subsection{3(3) VIew SImulation <filename> VAriable initial values}

This 1 ists all variables by category and index number. giving the initial values and system of units. The listing pauses each time the screen is filled. After the initial values are viewed the program does not return to the main menu, but to the VIEW SIMULATION menu, allowing another part of the simulation information to be viewed.

\subsection{3(4) VIew SImulation <filename> ERror tolerances and options}

This command displays the four error tolerances for the simulation, and the variable scan option and freeze option values for each superblock in the simu- 
lation. The significance of these tolerances and options is discussed briefly in section 3.6.3(2). After displaying the values, the program returns to the VIEW SIMULATION menu.

\subsection{3(5) VIew SImulation <filename> BOundary variables}

In response to this command, all of the boundary variables in the simulation are 1 isted. After the display the program returns to the VIEW SIMULATION menu.

\subsection{3(6) VIew SImulation <filename> REported variables}

This command displays the reporting interval and the reported variables for each superblock. After the display the program returns to the VIEW simulation menu.

\subsection{The Edit Command}

The CREATE and VIEW commands allow the creation or viewing of simulations, blocks, and units. The EDIT command, however, can only be used to EDIT units and simulations, not blocks. Any errors made in creating a block workfile can only be corrected after the block has been inserted into a simulation.

\subsubsection{EDit UNit <filename>}

The EDIT UNIT command can be used to change any of the information describing a particular unit, with the exception of the unit type. The EDIT UNIT command has slightly different capabilities depending on where it is used. If the command is invoked from the main menu, it can only be used to edit a previously created unit work file, and a file name is required. If the command is used while in the CREATE BLOCK or CREATE SIMULATION part of the program, its function is to edit any of the units that have been previously entered as part of the block or simulation work file being created. In this case no file name is required. Once the entry of a simulation using CReate SImulation is complete and the information has been saved in a work file, then EDit sImulation must be used to change any of the units.

The EDIT UNIT command can be used to produce a unit work file which is a modification of a previously existing unit work file. This is done by editing the original unit work file, making changes to the unit information, and then using a new filename to save the unit information. However, to create a new unit file of a different type, the CREATE DNIT command must be used.

If the EDIT UNIT command is entered from the main menu, a filename will be requested. This is an eight character name identifying a currently existing unit workfile. Alternatively the command may be entered in the form EDIT UNIT <filename>, in wich case the filename will not have to be requested. The EDIT UNIT command first causes a display of the unit information, in a manner much like the VIEW UNIT command. The display is divided into three numbered parts. Part one is the unit input variables, part two is the unit 
output variables, and part three is the unit parameters. These parts are the smallest portion of the unit information that can be selected for editing. The number of the part is used to select the part for editing when the program prompts for the "1ine number" to be edited. Note that the type of the unit cannot be selected for editing because the numbers of inputs, outputs, and parameters varies from type to type.

The "edit sequence" is used to change unit input or output indices or parameter values. For example, if inputs are selected for editing, the program displays the category and index for the first input and ask if the current value is acceptable (yes or no). A negative response (N) causes the program to ask for the correct index. The corrected value is echoed and acceptance or rejection is again required. When the value is correct, a carrage return (or Y) causes the next input to be displayed for approval. The edit sequence ends when all items have been approved.

After the complete group of inputs, outputs, or parameters has been produced and approved, the program again asks for a line number to edit. It is always true when editing units that one is inputs, two is outputs and three is parameters. If no additional editing of the unit is desired, a carriage return ends the editing and the information is saved in a work file with a "save sequence."

\subsubsection{EDit SImulation <filename>}

EDIT SIMULATION is used to change the information in an existing simulation work file. The name of the simulation file may be specified on the same line as the command such as EDIT SIMULATION <filename>. The program prompts for any information not given on the command line.

After the EDIT SIMULATION part of the program has been entered, the EDIT SIMULATION menu is displayed to allow any part of the simulation information to be edited. The choices are editing the title, editing initial values, editing boundary variables, editing reported variables, editing error tolerances and options, editing the simulation structure, and the most powerful of the choices, inserting and deleting (or replacing) units and blocks, and saving blocks. A final choice is to CONTINUE which returns the program to the main menu.

\subsection{2(1) EDit SImulation <filename> TItle}

This option allows the title of the simulation to be changed. The title may be used to record version numbers and dates, as well as a brief description of the simulation. In response to this command, the program displays the current title and asks if it is correct. If a No answer is given, the program prompts for a new title. After the new title is entered, it is redisplayed and the program again asks if the current title is correct. A Yes answer or a carriage return is entered if the title is acceptable. The program executes the "save sequence" if the title was changed, and then returns to the EDIT SIMULATION menu. 


\subsection{2(2) EDit SImulation <filename> STructure}

After the command is entered to edit the simulation structure, the program lists all of the units in the simulation, grouped in outline form. The blocks in each superblock are listed after the superblock headings and the units in each block are listed after the block heading. After the units are displayed, the program asks which unit is to be edited. This command allows any of the unit information to be changed except for the unit type. Changing a unit type must be done with the replace command. The operations to edit the unit are identical to those for the EDIT UNIT command. After editing is complete, the program asks for the number of another unit to edit. Another unit may be edited by entering its number. If a carriage return is entered, the program assumes that no more units are to be edited and goes through the save sequence if changes have been made to any unit. The program then returns to the EDIT SIMULATION menu.

\subsection{2(3) EDit SImulation <filename> VAriable initial values}

This command is used to change the initial value of any of the simulation variables. When this command is used, the program produces another menu containing all of the categories of variables (see table 2-1). If any of these categories is selected, the "edit sequence" is used to cycle through all of the current initial values for one variable category and allow any or all of them to be changed. After the variables in the selected category have been approved, the program returns to the Variable initial values menu and allows another category of variables to be selected for editing. To end editing of initial values and return to the EDIT SIMULATION menu, a carrage return may be entered when a new selection of variable category is requested. Before returning to the EDIT SIMULATION menu, the program goes through the "save sequence" if any initial values have been changed.

\subsection{2(4) EDit SImulation <filename> BOundary variables}

This command is used to change the variables designated as time dependent boundary variables in the simulation. The list of boundary variables is stored by variable category (pressure, temperature, and so forth). The boundary variable editing section of the program uses two functions to edit the boundary variable 1 ist: insert new boundary variables, or delete existing boundary variables. When the edit boundary variables command is selected, the current boundary variables are listed and a submenu displays the two choices. Figure 32 shows the possible paths that can be taken after entry of one of the menu choices.

The first option on the edit boundary variables sub-menu is insert boundary yariables. This choice first causes a display of the variable categories and one of these must be chosen by entering the appropriate number. The program then prompts for a variable index number. The program echoes the number received and asks if the value is acceptable. If not, the index may be reentered. The program will not accept an index higher than any which exist in 


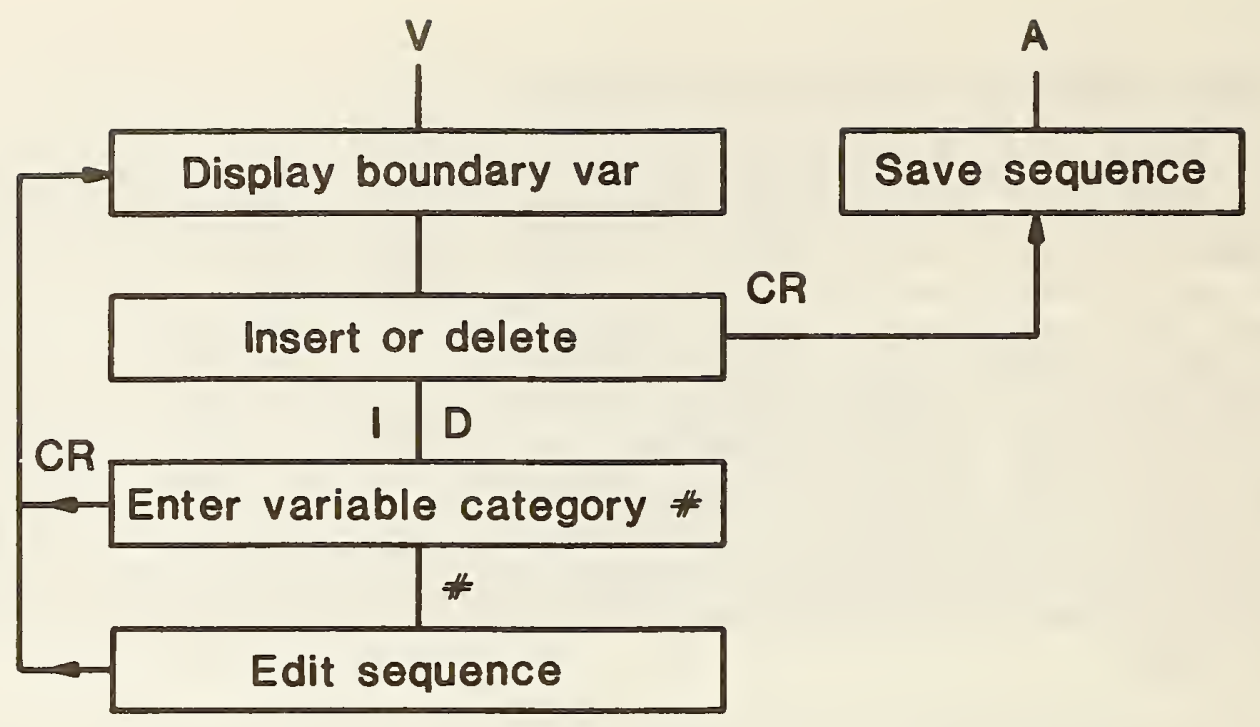

Figure 3-2. Menu flow path for editing boundary variables

the simulation. However, it will allow the same index to be entered more than once into the list. This dual entry causes the HVACSIM simulation program to expect two sets of boundary value inputs for the same variable. Care should be taken to ensure that all boundary variable indices are meaningful. After a single index has been inserted, a revised 1 ist of all boundary variables is displayed with the new index included. The program then returns to the edit boundary variables sub-menu. Insertion of several boundary variables requires several iterations of the entire procedure: selecting the insert function, selecting the category, entering the index, and verifying the entry.

The second option of the edit boundary variable sub-menu is delete existing boundary variables. The procedure is the same as that for inserting boundary variables. The program displays the possible variable categories and asks for a choice of variable category by number. Once the category is chosen, the program prompts for a variable index number. Once the index has been entered, the program displays the index that was entered and asks for confirmation that this is the correct index to be deleted. If not correct a value may be reentered. Note that the program will accept any variable index for deletion, whether it is currently a boundary variable or not. In this case there is no effect on the boundary variable list. After confirmation, a revised list of all boundary variables is displayed. The program then returns to the edit boundary variables sub-menu. Deletion of several boundary variables requires repetition of this procedure.

To exit the edit boundary variables command, a carriage return at the edit boundary variable sub-menu causes the program to return to the EDIT SIMULATION menu. A "save sequence" is performed before returning. 


\subsection{2(5) EDit SImulation 〈filename> REported variables}

The simulation program, HVACSIM, produces two output files of reported variables. One reports values at each time step actually taken by each superblock in the simulation. The other reports interpolated values at equal time intervals. The constant time interval may be different for each superblock. This command is used to change either the reporting interval or the list of reported variables.

When the EDit REported variables command is entered, the program displays the reporting interval and the list of reported variables for each superblock. Next it displays a sub-menu with three numbered commands: change the reporting interval, insert new reported variables, and delete existing reported variables. Figure 3-3 illustrates the flow of the program when any of the three choices is taken.

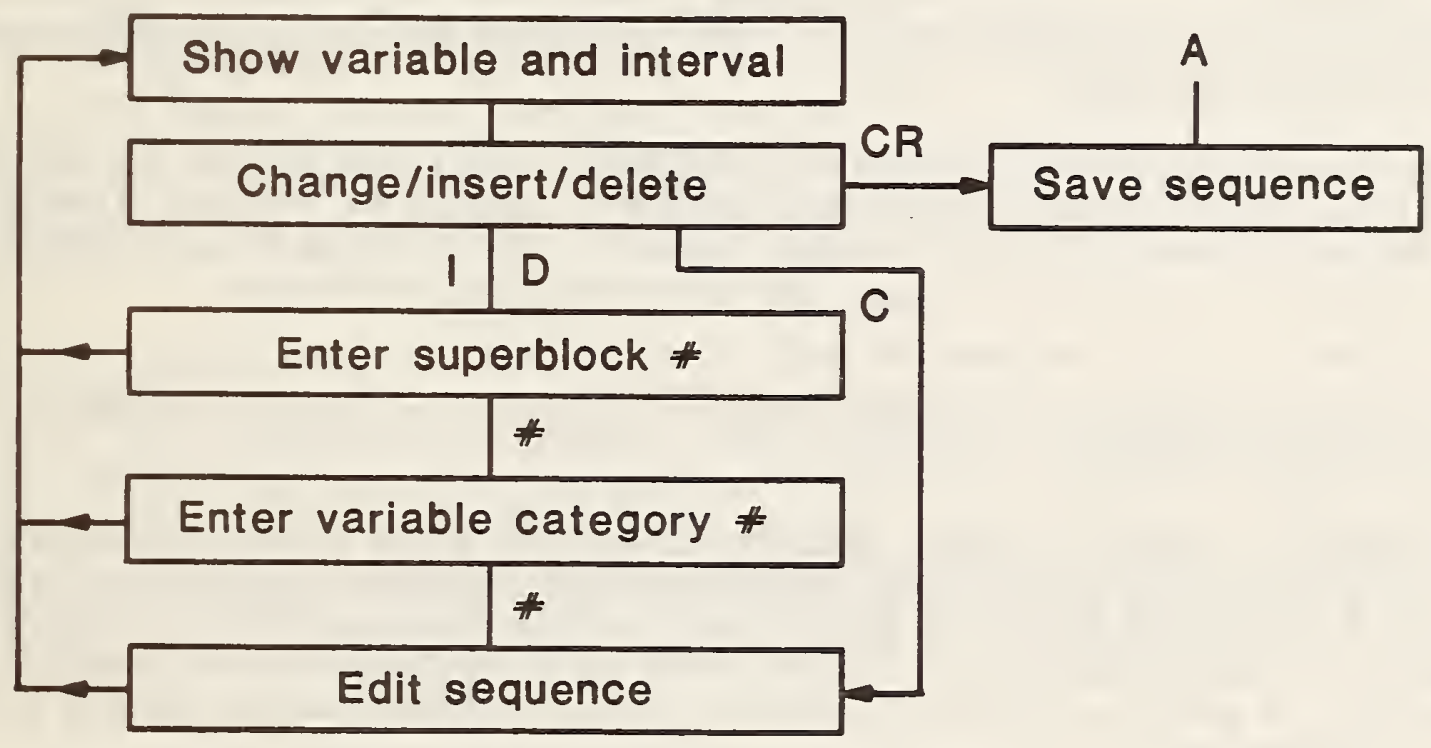

Figure 3-3. Menu flow path for editing reported variables

The change existing reporting interval sub-command is used to change the constant interval at which reported variables are written from the simulation for any or all of the simulation superblocks. Choosing this command invokes an edit sequence for all of the superblock reporting intervals. When the reporting interval for each superblock has been approved, the program again displays the reporting interval and list of reported variables for each superblock, and returns to the change/add/delete reported variable sub-menu.

The second and third options on the edit reported variables sub-menu are insert reported variables and delete existing reported variables. Selecting either of these options results in the following sequence: 
1. If the simulation contains more than one superblock, the program requests the number of the superblock associated with the variable to be inserted or deleted.

2. The program displays the available categories of variables, as given in Table 2-1, and requests a category number.

3. The program prompts for the index number of the variable to insert or delete.

4. The program echoes the index number entered by the user and requires approval (yes or carriage return) of this number.

5. The program displays an updated list of reporting intervals and reported variables for all superblocks, and returns to the EDit REported variables sub-menu.

This procedure can be very tedious when a long list of reported variables is to be replaced with a different list. The procedure may be modified in future versions of the program.

To exit the edit reported variables command, a carriage return at the edit reported variable sub-menu causes the program to return to the EDIT SIMULATION menu. Before returning to this menu, however, the program goes through the "save sequence" to save any changes in the simulation work file.

\subsection{2(6) EDit SImulation <filename> ERror tolerances and variable scan and freeze options}

This command is used to change certain parameters which affect the operation of the main simulation program. The explanation of these parameters may be found in section 3.6.3(2). If the choice is made to execute this command, a menu is first displayed which shows three options. These are: to edit error tolerances, to edit the variable scan and freeze options, and to return to the EDIT SIMULATION menu.

If the error tolerances are selected for editing, an "edit sequence" is executed which allows any or all of the four error tolerances to be changed. After the sequence, the program returns to the edit error tolerances and scan and freeze options sub-menu.

If the variable scan and freeze options are selected for editing, an "edit sequence ${ }^{\circ}$ is performed allowing the two options to be changed for each superblock in the simulation. After the sequence, the program returns to the edit error tolerances and scan and freeze options sub-menu.

To return to the EDIT SIMULATION menu, a carriage return is entered at the edit error tolerances and scan and freeze options sub-menu. This causes a "save sequence" and then the EDIT SIMULATION menu is redisplayed. 


\subsection{2(7) EDit SImulation <filename> INsert or replace}

There are two operations that change the units making up a simulation. An insert operation can place a new unit into the simulation at a selected location within an existing superblock and block. This unit may then be connected to other units by manipulation of the variable indices assigned to that unit. An insert operation can also place an entire new block into a simulation within an existing superblock. The replace operation, defined only for units, allows any existing unit to be replaced with another.

When the INsert command is chosen from the EDIT SIMULATION menu, a small submenu then allows the user to select the insertion of a unit, the insertion of a block or the replacement of a unit.

\subsection{2(7A) insert unit}

Before a unit can be inserted into a simulation, the block into which the unit is to be inserted must be specified. The program prompts for a block number, echoes the number entered, and asks for confirmation that the number is correct. A yes (carriage return) or no answer is required. If a block number which is not in the simulation is entered and verified, the program prints a message that the block is not in the simulation and asks for another block number.

Next the program asks for the source of the information about the unit that is to be inserted into the block. This unit information can come from one of two sources. One source is to enter all unit information directly from the keyboard. This is called creating, and is the same as creating a unit under the CREATE UNIT command. The other source of unit information is an existing unit work file previously created with the CREATE UNIT or EDIT UNIT commands. When the unit is entered, the new unit is given the next unit number in sequence in the simulation, regardless of the unit numbers of the other units in the block where the insertion is made.

To enter a unit from the keyboard, select option 2 when the source of the inserted unit is requested. The entry of unit information is the same as for the CREATE UNIT command (see section 3.6.1). The program calls for the entry of the unit type, input and output variable indices, and parameter values. After the last parameter is entered a warning message reminds the user to check the inputs and outputs carefully. Each input or output variable index that is entered is used to connect the unit to other units in the simulation. The program also prints a reminder that the initial values, reported variables, and boundary variables may need to be viewed/edited after the insertion of this unit since new variables may have been added with the unit. Editing of the newly inserted unit or the initial values can be easily performed by returning to the EDIT SIMULATION menu and editing the appropriate items.

After the unit has been completely entered, the program executes a "save sequence" and returns to the INsert or replace sub-menu. This allows several units or blocks to be added in succession without returning to the EDIT 


\section{SIMULATION menu.}

To read a unit from a unit work file, select option 1 when the source of the inserted unit is requested. The program then prompts for a filename. This name must be the eight character name which represents an existing unit work file. Note that the inputs, outputs, and parameters for the unit being inserted will be the same as they were when the unit work file was created. The parameters are likely to be correct since the unit may be constructed to represent a specific piece of equipment, but the inputs and outputs are probably incorrect at this point. Before reading the work file, the program asks if all variable indices are to be set to zero. Answering yes to this question effectively inserts the unit as a completely disconnected unit with no connections to any other unit. The EDIT SIMULATION STructure command must be used to modify these values to correctly connect the unit to the other units in the simulation (see section 3.8.2(2)). If the indices are not set to zero, they are set to the values stored in the unit work file. In either case, a warning message is written to remind the user to edit the unit inputs and outputs, and to view or edit the initial values, and possibly the boundary and reported variables.

After the unit work file is read, the program goes through a "save sequence" to save the enlarged simulation in a simulation work file, and returns to the INsert or replace sub-menu. A carriage return brings the program back up to the EDIT SIMULATION menu.

\section{$3.8 .2(7 \mathrm{~B})$ insert block}

Before a block can be inserted into a simulation, the superblock into which the block is to be inserted must be specified. The program prompts for a superblock number, echoes the number entered, and asks for confirmation that the number is correct. A yes (or carriage return) or no answer is required. If a superblock number which is not in the simulation is entered and verified, the program prints a message that the superblock is not in the simulation and asks for another superblock number.

Note that blocks can only be inserted into existing superblocks. It is not possible with the current version of the program to increase the number of superblocks in an existing simulation. To expand the number of superblocks in a simulation, CREATE SIMULATION can be used to create a simulation with the correct number of superblocks and with only one dummy block, containing one dummy unit, in each superblock. The TYPE 26 component is convenient for this purpose, since it has only one input, one output, and no parameters. The blocks to make up the simulation can then be read from block work files. It is strongly suggested that a complex simulation be built from block work files for maximum flexibility. Block work files can be prepared using either the CReate BLock command (section 3.6.2) or the SAve command described below in section $3.8 .2(9)$.

Once the superblock into which insertion is to take place is identified, the program asks for the source of the information about the block. This block information can come from one of two sources. One source is to enter all 
information directly in from the keyboard. This is called creating, and is similar to creating a block under the CREATE BLOCK command. The other source of block information is an existing block work file. When the new block is entered, all of the blocks in the simulation are renumbered according to their location in superblocks. The inserted block is given a number one greater than any of the other blocks in the superblock into which it was inserted. Blocks in superblocks with numbers higher than the superblock where the block was inserted will have their block numbers incremented by one. Blocks in superblocks with numbers lower than the superblock where the block was inserted will have unchanged block numbers. Thus, unlike unit numbers, block numbers are always sequential with the lowest block numbers in superblock one and the highest block numbers in the highest-numbered superblock.

To enter a block from the keyboard, select option 2 when the source of the inserted block is requested. The entry of block information is very similar to the entry of information when creating a block (see section 3.6.2). The difference between inserting a block from the keyboard and CREATE BLOCK is that the number of units to be included in a block inserted from the keyboard must be specified first. The program then prompts for information on this number of units. The program calls for the entry of the unit type, input and output variable indices, and unit parameters. After the last parameter is entered a warning message reminds the user to check the inputs and outputs carefully. Each input or output variable index that is entered is used to connect the unit to other units in the simulation. The program also prints a reminder that the initial values, reported variables, and boundary variables may need to be edited after the insertion of this block since new variables may have been added with the block. Editing the newly inserted block or the initial values can be easily performed by returning to the EDIT SIMULATION menu and editing the appropriate items. After the block has been completely entered, the program executes a "save sequence" and returns to the INsert or replace sub-menu.

To read a block from a block work file, select option 1 when the source of the inserted block is requested. The program then prompts for a file name. This name must be the eight character name which represents an existing block work file. If an illegal or nonexistent file name is entered, the program identifies the error to the best of its ability and repeats the request for a file name.

Before reading the work file, the program asks if automatic renumbering of variable indices desired. Answering yes to this question effectively makes all of the variable indices in the inserted block unique in the simulation by adding an offset to each variable index. The offset for each category of variables is equal to the highest current index for variables of the same category. For example, if there were 17 pressure variables in the simulation, and a block were being inserted which had pressures 2, 3 , and 4 within it. after renumbering and insertion, this block would contain pressures 19, 20, and 21. The renumbering leaves the block unconnected to the rest of the simulation but leaves the internal structure of the block intact. The HVACGEN program does not determine whether there are variable indices that are not used below the maximum index in any category. Block indices are always renumbered above the highest index in the simulation. 
If the automatic renumbering option is not selected, input and output indices are taken directly from the block work file without modification. In either case, parameter values are read from the block work file without modification.

After the block information is read from the file, a warning message is written to remind the user to edit the unit inputs and outputs, and, in addition, to edit the initial values, and possibly the boundary and reported variables. Then the program goes through a "save sequence" to save the enlarged simulation in the simulation work file, and returns to the INsert or replace sub-menu. A carriage return brings the programback up to the EDIT SIMULATION menu.

Generally, after a block has been inserted, the EDit SImulation STructure command must be used to modify input and output variable indices in the inserted block to correctly connect the units in the block to the other units in the simulation (see section 3.8.2(2)). The EDit SImulation VAriable initial values command (section $3.8 .2(3)$ ) should also be used, to provide initial values for newly defined variable indices. Often it is also desirable to modify the 1 ist of reported variables.

\subsection{2(7C) replace unit}

To replace a unit, the replace unit operation is chosen after invoking the INsert command from the EDit SImulation menu. The program then prompts for the unit to be replaced. The unit number to be entered here may be obtained from the diagram worksheet of the simulation or by using the VIew SImulation STructure or EDit SImulation STructure commands.

After the unit number to be replaced is entered, the replace unit operation is identical to the insert unit except that after the replacement is finished the program returns to the EDit SImulation menu rather than the INsert sub-menu. The source of the unit information can be the keyboard or a previously created unit work file (see INSERT UNIT command, section 3.8.2(7A)). The net effect of the replace unit command is to change the component type number associated with a specified unit number.

\subsection{2(8) EDit SImulation <filename> DElete}

The DELETE command is used to completely remove a unit or a block from a simulation. When the DELETE command is selected, the program displays a small sub-menu which gives a choice of deleting a unit or deleting a block.

When a block or unit is deleted, all variables that were uniquely in that block or unit are removed from the simulation. For a block, all internal block variables, or all those variables not connecting the block to other blocks, will be removed from the simulation work file after the block is deleted. The removal of variables applies to the initial values, boundary variables, and reported variables. All references to variables in the deleted block are automatically deleted. 


\subsection{2(8A) delete unit}

If the unit option of DELETE is selected, the program prompts for the number of the unit to be deleted, echoes the unit number entered by the user, and asks for confirmation that this unit is to be deleted. It is not possible to delete a unit if it is the only unit in a block. However, the last unit in a block can effectively be removed by deleting the block in which the unit is found. If the unit number does not exist in the simulation, or if the unit cannot be deleted, the program writes an error message and repeats the request for the unit number to be deleted.

After deleting the unit, the program immediately decrements the unit numbers of all units greater than the deleted unit. For example, in a simulation with ten units where unit five is deleted, the old unit six will afterwards have the identification number five, the old unit ten will have the unit identification number nine, and there will be no unit numbered ten. Units originally numbered one through four will have the same numbers. This is done because the main simulation program does not allow any gaps in the sequence of unit numbers. The highest unit number in the simulation is always equal to the total number of units.

After the unit is deleted and the remaining units are renumbered, the program executes a "save sequence," and returns to the DElete sub-menu.

WARNING: since units are renumbered immediately after each delete operation, deleting multiple units can be hazardous. To avoid confusion, always delete the highest-numbered unit first and the lowest-numbered unit last when more than one unit is to be deleted. If a unit is deleted by mistake, the ABort command may be entered when the filename confirmation is requested, and the most recent deletion will effectively be restored. Alternatively, the file with the accidental deletion can be saved under a different name, allowing recovery of the original file. When in doubt, the EDit (or VIew) sImulation STructure command can be used to review unit and type numbers.

\subsection{2(8B) delete block}

If the block option of DELETE is selected, the program prompts for the number of the block to be deleted. Any block number up to the total number of blocks in the simulation may be entered. The user must be careful to select the correct block for deletion. If the number is uncertain, the EDit (or VIew) SImulation STructure command can be used to pinpoint the correct block. The program echoes the block number that was entered and asks for verification that this block is to be deleted. Entry of an incorrect block number causes an error message to be displayed. If the block chosen is the only block in a superblock, the program informs the user that there is only one block in the superblock and it may not be deleted. The current version of the program does not allow a superblock to be removed from a simulation by deletion. An existing simulation can only be cut down to one block in each original superblock, and the blocks can only be cut down to one unit. 
When a block is deleted, the block numbers of the other blocks in the simulation may be changed. In addition, unit numbers of all units in the simulation may be changed. If a block is deleted, blocks with identification numbers higher than the deleted block will have their identification numbers decreased by one. Blocks with numbers lower than the deleted block will have the same numbers as before the deletion. When a block is deleted, obviously all of the units in the block are also deleted. For each unit in the deleted blocks, all units in the entire simulation which had unit identification numbers higher than the removed units will have their numbers decreased by one. Units with numbers lower than any of the units in the deleted block will have the same numbers after the deletion. For example, consider a simulation having two blocks with the first block containing units 2, 4, and 6 , and the second block containing units 1,3 , and 5. If the first block were deleted, block 2 would be numbered as block 1. Units 1, 3, and 5, would then have numbers 1, 2, and 3 .

\subsection{2(9) EDit SImulation <filename> SAve}

The SAve command is used to produce a block work file which contains the block description information for a block which currently exists within a simulation. The block work file produced by the SAve command is the same as a block work file produced by the CReate BLock command. The saved block work file may be inserted in the existing simulation in a different place or inserted into another simulation.

If the save command is entered, the program prompts for the number of the block to be saved. This must be a block within the current simulation being edited. An edit sequence allows the block number to be checked and changed if necessary. Once the block to be saved is selected, the program prompts for the name of a file into which to save the block information. The default filename is the same as the simulation work file name, but this can be changed to any other name. After the save is complete, the program returns to the EDit SImulation menu.

\subsection{The Help Command}

The Help command gives several pages of summary information. It may be invoked from anywhere in the program, at any prompt for input.

\subsection{Producing a Model Definition File}

HVACGEN is used to create and edit files containing enough information to configure a simulation. These files are called work files and are not directly usable by the HVACSIM simulation program. HVACSIM must have as input a model definition file which contains the information in the work file in a modified form. A separate utility program called SLIMCON must be used to convert simulation work files into model definition files. SLIMCON also has several error-checking functions, such as examining the values stored in the work file to ensure that the simulation model does not exceed the currently 
configured capabilities of the HVACSIM simulation program in terms of number of units, number of variables, and other similar measurements.

The operation of the SLIMCON program is very simple. When the program is run, it prompts the user for the name of a simulation work file. The name is compatible with the naming conventions for work files used within HVACGEN. If no name is entered (a carriage return), then the program ends. If a simulation work file is found with the specified name, then the program reads the work file, analyzes the information in the work file, and creates the model definition file. As distributed, SLIMCON gives the model definition file the same name as the simulation work file but with the extension. .DAT in place of the extension .SIM

During the execution of SLIMCON, a table is written which gives a description of the simulation. An example of a possible table is given below:

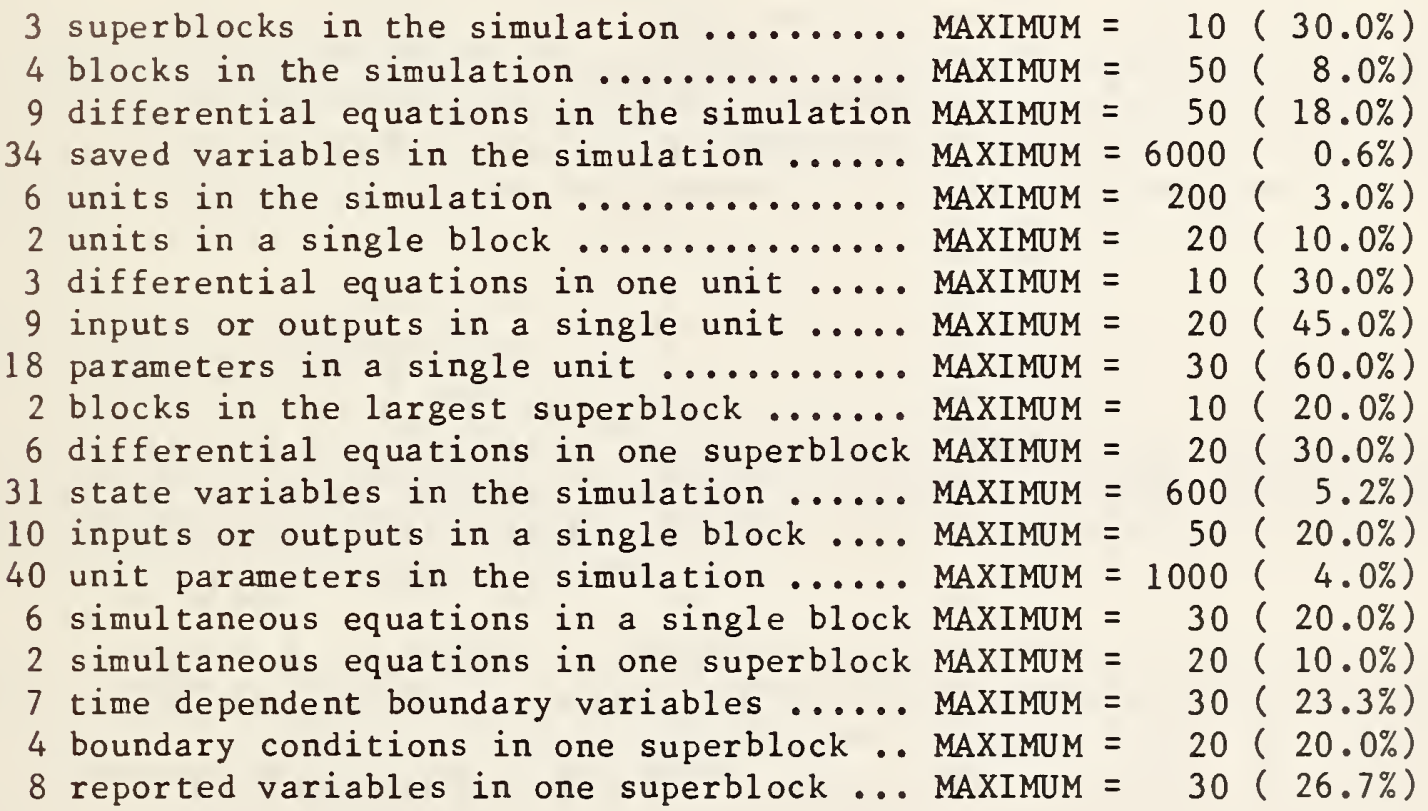

The program HVACSIM and the program HVACGEN are configured to have certain maximum sizes for the quantities listed in the table above. These maximum sizes are determined by array dimensions which are specified by PARAMETER statements in the source code of each program. HVACGEN performs only a limited amount of checking of these quantities to see if they exceed the maximums and therefore it is possible to configure a simulation which may not run or which may yield incorrect results. SLIMCON performs a check on the quantities listed above and 1 ists the number computed from the simulation work file and the currently configured maximum together with a percentage of the maximum that the current simulation has reached. If any maximum is exceeded, an error message is written. If a maximum is approached closely, a warning message is written.

If an error message is observed the user has two choices. The first choice is 
to edit the model to lower the size of the offending parameter. In the case of reported variables, for example, this is not difficult. The other choice is to reconfigure the HVACGEN and HVACSIM programs to a larger size (see installation guidelines in chapter 5). If this choice is made, the corresponding PARAMETER values in the SLIMCON source code should also be changed. 


\section{PREPARING AND RUNNING SIMULATIONS USING HVACSIM ${ }^{+}$}

Generally, a computer simulation using HVACSIM ${ }^{+}$involves three steps: preprocessing. execution, and post-processing. This chapter summarizes the necessary procedures in each of these three areas.

\subsection{Pre-Processing}

When HVACSIM is run, it reads data from one or more files which must be prepared in advance. Most of the input files are optional, and need not be present for all simulations. The various required and optional input files are described in this section.

\subsubsection{The Model Definition File}

The primary input to HVACSIM is the model definition file. This file is the output of the program SLIMCON, which takes as input a simulation work file prepared by the simulation editor program, HVACGEN. The procedures for generating the simulation work file and the model definition file are discussed extensively in chapter 3. A model definition file is always required to run a simulation.

\subsubsection{The Boundary Variable File}

Boundary variables which have been declared time-dependent in configuring a simulation must be assigned values which are stored in a boundary value file. As the simulation progresses, data are read from this file and are used to change the appropriate state variables.

The first column of the boundary variable data file must contain values of time. Time intervals need not be equal. The remaining columns contain values of the boundary variables at the specified times. HVACSIM begins all simulations at time zero regardless of the time on the first entry in the boundary value file. To avoid confusion it is recommended that boundary data files always begin with time zero.

To produce discontinous changes in boundary variable values, a special reset convention is used in the boundary value file. When two sequential records have the same value of time but different values of a boundary variable, the program will correctly generate a step change in the boundary variable. The second record contains the value to which the variable is to step. At least three regular records must occur between reset record pairs.

The reset convention can also be used to mark a discontinuous change in the slope of a boundary variable, such as the beginning of a ramp input. In either case the reset convention serves to notify HVACSIM that the simulation time step should be reduced to its minimum value when the specified time is reached. Both of these uses of the reset convention are demonstrated in the first example in Chapter 6. 
The simulation program always opens the boundary file, but does not attempt to read from this file if no variables have been declared time dependent.

\subsubsection{The Initialization File}

Each time HVACSIM is run, a complete discription of the final state of the simulation is written to a file. An initialization option has been included which allows the final state of one simulation to be used as the initial state of another. This means that a series of simulations of a given system can be run from the same initial steady state, without having to repeat a computationally expensive startup transient for each run. Also, long simulations can be run in several parts, with each part beginning precisely where the previous part ended. Since MODSIM begins all simulations at time zero, if a long simulation is to be run in several parts it will be necessary to prepare a separate boundary value file starting at time zero for each part.

The initial state file is not required, and the initialization option cannot be used the first time a newly prepared simulation is run. The contents of the initial state file are discussed in section 6.2 .

\subsubsection{The Conduction Transfer Funtion File}

The conduction transfer function (CTF) file is required only when the TYPE 51 component is used in a simulation. The TYPE 50, TYPE 51, and TYPE 52 components together constitute the "building shell model." The characteristics and use of these components are the subject of a separate document [2]. The CTF file is produced by the program CTFGEN, and contains transfer functions defining the thermal properties of building constructs such as walls, windows, roofs, and floors or ceilings.

\subsubsection{The Weather Data File}

The weather data file is used only by simulations containing the "building shell model" consisting of TYPE 50, TYPE 51, and TYPE 52 component models. This file is generated by the program CRWDTA, which may be used alone or in conjunction with the program RDTAPE. RDTAPE is designed to read any of several readily available weather data tapes and to write a portion of the tape to a file in a fixed format and system of units. CRWDTA allows several options. It can read the output of RDTAPE and rewrite the information in the format required by HVACSIM, dividing total horizontal solar radiation values into beam and diffuse components if necessary. Alternatively, it can generate smooth "design day" solar radiation and temperature data by any of several methods. These programs are described more extensively in reference [2]. When the building shell model is used, the weather data file supplies the simulation program with outdoor conditions of temperature, humidity, barometric pressure, wind speed, and solar radiation. Other time dependent boundary conditions such as occupancy schedules and equipment setpoints are supplied separately through the boundary variable file. 
When the building shell model is not used, all time dependent boundary conditions, including any weather data, must be supplied by the boundary variable file.

\subsection{Execution}

When the simulation program is run, it prompts the user for a minimum time step, a maximum time step, and a stopping time. The choice of minimum and maximum time steps influences the accuracy and stability of the simulation, as well as the amount of computation required. Typical values are 1 second for the minimum time step and 3600 seconds (one hour) for the maximum time step.

The program also prompts the user for some additional information. The questions listed below are from Version 4.7 of the simulation program. The precise order and content of the questions may be slightly different in later versions of the program.

1) Will the Initialization File be called?

A "Yes" answer causes the initial state of the system to be read from the initialization file. Otherwise, the initial state vector in the model definition file defines the initial state of the system.

2) Use default file names for all files?

If an answer of "No" is entered, the user is prompted to enter a name for each input and output file, or a carriage return if the default name is to be used for that file.

3) Do you want diagnostic information to be written?

If "Yes" is entered, the user is given several choices as to what information should be written (including "No diagnostics"*). The choices all relate to the freezing and unfreezing of state variables and the progress of the equation solver. Starting and stopping times for diagnostic output are also requested. The selected category of information is written to the "simulation summary File" with the default name MODSUM.DAT . This feature can be useful for debugging, but may produce large quantities of output. Normally diagnostics are not required, and only final values at each reporting time are written.

4) Would you like to monitor the Simulation Time?

A "Yes" answer causes information relating to the simulation time and time step size to be written to the console or other output device as the simulation progresses.

\subsection{Post-Processing}

Two kinds of reports are generated by HVACSIM: a "data file" (default name 
MODOUT.DAT) containing raw data at each time step taken by each superblock during the simulation, and a "summary file" (default name MODSUM.DAT) containing a summary of the simulation configuration, any diagnostics generated during the computation, and an interpolated listing of the reported variables at equal time intervals. In addition, an output file containing the final state of the simulation (default name INITOUT.DAT) is produced. This last file can be renamed and used as the initial state file (default name INITIN.DAT) for subsequent simulation runs.

In the summary file, variables are reported to three significant digits and each reported variable is identified by a label. This file is intended to be easy for the user to read. The data file reports each variable to six significant digits, without labels, and is more suitable for further processing by a computer.

Reports are generated for each superblock in the simulation individually as the simulation progresses. For simulations with more than one superblock, reports for the various superblocks are intermingled in an unpredictable order. To facilitate further processing of the output, a simple program called SORTSB is provided. This program reads the file MODOUT.DAT and writes a new file containing the times and variables reported by any or all superblocks, separated by superblock number. For example, all the times and variables reported by superblock 3 could be extracted, followed by all the times and variables reported by superblock 2. Simulation times can also be converted from seconds to another time unit such as minutes or hours. The resulting file is more readily used as input to user-supplied post-processing software such as a data plotting program. No graphics software is supplied with HVACS IM ${ }^{+}$. 


\section{INSTALLATION OF THE HVACS IM PROGRAMS}

The HVACSIM programs are distributed as source code files, usually on magnetic tape. All programs are written in the FORTRAN computer language. An effort has been made to conform as much as possible to ANSI X3.9-1978 FORTRAN (FORTRAN 77). The installation of the programs includes the following activities: modification of the source code to reflect differences in host computers. compilation of programs, linkage to produce executable programs, and creation of procedures for executing the programs.

\subsection{HVACSIM distribution files}

The HVACSIM source code as distributed consists of 33 files. When the files are read from a magnetic tape, they should be given the names shown below (or equivalent names if the form of the names is incorrect for the target computer).

\begin{tabular}{|c|c|}
\hline HV ACS IM. DOC & 12. SELECT5.FTN \\
\hline MODS IM5.DOC & 13. TYPES.FTN \\
\hline SNSQ.DOC & 14. UTIL ITY.FTN \\
\hline MODSIM5.FTN & 15. WATPR.FTN \\
\hline MODIN 05 .FTN & 16. AIRPR. FTN \\
\hline MODBLK5.FTN & 17. REFR IGPR.FTN \\
\hline MODBDF 5 . FTN & 18. TYPESB.FTN \\
\hline MODEQT5.FTN & 19. UTILITYB.FTN \\
\hline SNSQA. FTN & 20. RDENV.FTN \\
\hline 0. SNSQB.FTN & 21. THERM.DAT \\
\hline SNSQC.FTN & 22. CTFGEN.FTN \\
\hline
\end{tabular}

23. CRWDTA. FTN

24. RDTAPE. FTN

25. SORTSB. FTN

26. SLIMCON.FTN

27. TYPAR.DAT

28. HVA CG EN1 . F TN

29. HVACGEN2. FTN

30. HVA CGEN3 . FTN

31. HVACGEN4.FTN

32. HVACGEN5.FTN

33. HVACGEN6.FTN

The first file contains some documentation for the HVACSIM package, and is a subset of the information in this chapter with minor exceptions. The file on the tape contains the version numbers and date last modified for the source code files on the tape, and provides some information about support of the programs. The second and third files provide some documentation for the main simulation program (MODSIM) and the non-linear equation solver (SNSQ) called by MODSIM.

Files 4 through 20 make up the components of the main simulation program, HVACSIM. The name MODSIM refers to files 4 through 12, which make up a general-purpose modular simulation program. Files 13 through 20 contain the models used in simulating heating and cooling systems. Files 13 through 17 are the component models and utilities described in reference [1], and files 18 through 20 constitute the building loads model described in reference [2].

File 21 is a data file read by the program in file 22. Files 22 through 26 are utility programs. Files 21 through 24 are used in conjunction with the building model (see sections 4.1.4 and 4.1.5). File 25 is the simple postprocessing program mentioned in section 4.3. File 26 is the source code of the utility program SLIMCON, which reads simulation work files and "compiles" the information into model definition files. 
File 27 is the types description file. This is an ASCII file which is used by HVACGEN and SLIMCON during execution. It contains the information these programs require about the TYPE subroutines. When user-supplied component models (TYPE subroutines) are added to the component library, the information in this file must be updated. Files 28-33 make up the components of the HVACGEN program, used to prepare model description work files.

The total number of lines or records (including comment lines) contained in the above $32 \mathrm{files}$ is approximately 1000 for the document files, 11,000 for the HVACSIM simulation program, 1000 for the SLIMCON program, 3000 for the other utility programs, and 8000 for the HVACGEN program, for a total of approximately 24,000 1 ines of source code. The HVACSIM source code alone occupies approximately 540 kilobytes (ASCII characters) when records are not padded to 80 bytes with nulls or spaces. The HVACGEN source code occupies approximately 370 kilobytes.

On the computer used to develop the programs, the executable code produced from the source code of HVACSIM as distributed requires approximately 300 kilobytes of memory plus a portion of a 100 kilobyte memory resident FORTRAN library. HVACGEN also requires approximately 300 kilobytes of memory plus a portion of the library. SLIMCON requires approximately 100 kilobytes of memory plus a portion of the library. Note that these programs do not need to occupy memory simultaneously.

\subsection{Installation Process}

The first step in the HVACSIM installation process is to make any source code modifications necessary for the HVACSIM programs to run on specific computers. Each of the programs in the package must be successfully compiled and the resulting object or binary files must be linked with a FORTRAN library to produce executable programs. The following three sections describe areas in the source code of the three major programs in the package (HVACSIM, SLIMCON, and HVACGEN) which may require modification.

\subsubsection{HVACSIM source code compilation and linkage}

The source code for the main HVACSIM simulation program should be system independent and should compile on most computers without modification, with three exceptions. These are:

1. The logical unit assignments may have to be changed for a specific system. The assignments are found in a BLOCK DATA subprogram which is the first subprogram in the source code file MODIN05.FTn. Common Block FILES contains the logical unit numbers. On some systems, reading and writing to the console may require the use of an asterisk for the logical unit number in read or write statements. In this case, all console read and write statements must be edited to change the logical unit variable to an asterisk. Print statements used for console output may have to be changed for some systems. 
2. The file names used in the file OPEN statements may be illegal under some operating systems and may have to be changed before compilation. The file names and OPEN statements are located in subroutine OPNFIL, the second subprogram unit in the source code file MODIN05.FTN.

3. A function named RIMACH located in file SNSQC.FTN must be customized for the particular type of computer on which the program is being run. This function is used to set machine constants. The function can return any of five values, but only two are used by HVACSIM: RlMACH(2) returns the largest positive real number the computer can represent, and R1MACH(4) returns the machine precision. Values for a number of computers are included in the function as distributed and if the target computer is in this set, the customization involves commenting out all lines except those for the target computer. If the target computer is not in the supplied group, the installer will have to determine the parameters for the target computer.

Files 4-20 as 1 isted in section 5.1 should be compiled with a FORTRAN 77 compiler to produce object files. To link the HVACSIM program, object files MODSIM5, MODIN05, MODBLK5, MODBDF5, MODEQT5, SNSQA, SNSQB, SNSQC, SELECT5, and RDENV (files 4-12 and file 20) should be completely included in the link. The object files TYPES, UTILITY, WATPR, AIRPR, REFRIGPR, TYPESB, and UTILITYB (files 13-19) should be searched as library files to only bring in the subroutines needed.

All calls to TYPE subroutines are located in the subroutine SELECT, which is the only subroutine in the file SELECT5. If necessary, the size of the executable program can be reduced by commenting out calls to any TYPE subroutines that are not needed by the user. Removing references to TYPE subroutines may also eliminate references to many of the functions and subroutines in files 14-17 and 19, which are used only by the TYPE subroutines.

\subsubsection{SLIMCON source code compilation and linkage}

SLIMCON.FTN should be compiled with a FORTRAN 77 compiler and only 1 inked with a FORTRAN library. The logical unit assignments may have to be changed for specific computers. The file name coding assumes the existence of a file name extension to indicate the type of file. Parameter statements are included in the program to customize the file naming conventions for specific computer operating systems.

\subsubsection{HVACGEN source code compilation and linkage}

Files 28-33 as 1 isted in section 5.1 should be compiled with a FORTRAN 77 compiler. There are a few system dependent sections of the program which may have to be changed for specific computer systems. These are (all specific 1 ine numbers listed below are for HVACGEN version 1.8 and may be approximate for other versions or updates): 
1. The logical unit assignments are contained in variables in the common block LOGUNT. The specific assignments are made in the main program, located in the file HVACGEN1.FTN on lines 119 - 123 of the file. For some computer systems, an asterisk may be required for the logical unit number of all read or write statements. In this case all occurences of the variable LUC in all source code files in read and write statements will need to be changed to an asterisk.

2. The subroutine SCROLL in HVACGEN1.FTN ( 1 ines 715-736) is used to cause a pause after a certain number of 1 ines have been written to the computer terminal. The default number of lines is 18 which is correct for a 24 line terminal. The number of lines is located in a PARAMETER statement.

3. The naming, opening and closing of work files is performed in the subroutine OPNFIL in HVACGEN3.FTN ( 1 ines 108-329). There are four parameters which must be set in PARAMETER statements to allow the program to successfully open and encode work file types into file names. Three of the parameters are concerned with file naming conventions and one of the parameters enables or disables a backup file system. Comments in the subroutine explain the selection of parameters. In addition, any variations in file OPEN statements for specific systems should be corrected here. All OPEN statements are FORTRAN standard with the exception of line 319 which is used in the backup file system. The backup file system is optional and the code for it may be commented out of the source code.

4. All access to the types data file (TYPAR.DAT) is through the subroutine TYPEIN in HVACGEN3.FTN ( 1 ines 1070-1223). The name of the types data file is specified in a PARAMETER statement, and may require modification for some operating systems.

\subsection{Description of HVACGEN subroutines}

The HVACGEN source code is subdivided into sections which will be referred to as modules. The division into modules reflects the the commands displayed at the main menu when running the program. There are CREATE, VIEW, EDIT, and HELP modules as well as a FILES module. The following descriptions will give details of the purpose of each subprogram within the modules.

\subsubsection{HVACGENl - main module}

The first source code module is the main module. This module includes a block data subroutine (INFORM) with data statements for all common blocks in the program. Also in the main module are the routines for the main menu display and command processing. The assignment of specific logical unit numbers for reading and writing to the console and files is found in this module.

The DATAIN subroutine contains the command processor and console input routines. This routine allows a single logical procedure call to get a command or number from the program user. DATAIN allows printing of prompt messages, verification that the information obtained is the type of information request- 
ed. limit checking for numerical input, and entry of commands and sub-commands on a single line. After calling DATAIN, ITEM is the first piece or word from the string that was entered by the user, ANUMBR is the real number equivalent of ITEM, if it was a number, and INTNUM is the integer equivalent. Other input arguments specify what the calling routine requires from the user (eg. a word, a number with certain limits, etc.).

DATAIN calls COPMOD (command process module) which contains the only console read statement in HVACGEN. This module reads the line typed by the user into a character variable (LINE). The line is parsed into words (groups of characters separated by spaces or commas) and each time the routine is called, the next word in the line is stored in a character variable (ITEM).

Subroutine CHECK is used to determine whether the parsed word stored in ITEM is a number. A word in ITEM consisting of all numbers, plus signs, minus signs, and similar characters with certain constraints is judged to be a number. Otherwise it is assumed to be a command or name.

Subroutine REWORD is called by DATAIN and is used to determine if the item entered was a reserved word (command). If it was, the usual check to see if the item entered is the same type of information as what was requested is bypassed. This allows a user to enter some commands such as ABORT, at any time.

REMAIN is the subroutine that determines which module will be executed in response to a command from the console. It is called by MAIN after a command is read. Every major program unit such as CREATE, VIEW, etc., includes a subroutine of this type. The subroutines can be identified by the name which begins with the letters "RE," followed by the module name. After a command has been executed, the control is returned to the MAIN module.

The main module contains a number of utility routines used throughout HVACGEN. The subroutine HOLDIT is used to produce a pause for acknowledgement after an error message is displayed before clearing the screen. SCROLI is called by routines displaying tables of information on the screen and causes the program to pause for acknowledgement after a fixed number of lines has been displayed on the console. OKAY is used to determine the answer to a yes or no question as to whether an existing value is acceptable and, if the value is unacceptable, allows the value to be changed. The subroutine RITE allows the display of console messages and menus, line by line, and allows the text of a message to be easily edited or examined in the source code. PROMPI is called to determine the alphanumeric name corresponding to a variable category number.

\subsubsection{HVACGEN2 - create module}

The first subroutine in this section is CREATE, which is the pathway to the creation of unit, block, or simulation work files. CREATE is called by MAIN if the command CREATE is entered. CREATE calls DATAIN to get the subcommands to CREATE. RECRT is called immediately after receiving a subcommand to determine if the item entered was a special reserved word for help, editing, or viewing. Note: If a RExxx subroutine is not called after DATAIN is called, no alternative commands may be entered. 
CRUNT is the module for creating a unit. It contains all the queries and responses for selecting the inputs and outputs and parameters for a unit. CRBLK is used to create blocks. CRBLK calls CRUNT to get information for each unit in the block. CRSUP is used to create superblocks. It, in turn, calls CRBLK. CRSIM is used to create entire simulations. It calls CRSUP and a number of other routines to define a simulation. CRSIM contains the code to determine the total number of various categories of simulation variables. It checks for the highest index value used for any of the variable category arrays. This subroutine also gets the boundary values, initial values, and reported variables. The routine is broken into five parts named CRSIM, CRSIM1, CRSIM2, CRSIM3, and CRSIM4. RECRT is used for detecting the entry of reserved words if the help, view, edit, types, or abort commands are entered.

The subroutine TYPES is used to display the numbers and names of the types in the types information file. It is called when the TYPES command is used in response to a request for a type number.

\subsubsection{HVACGEN3 - file control module}

The file control module is used to read and save information in work files and to access the data in the types information file. The FSAVE subroutine is called by the create and edit modules whenever the current unit, block, or simulation information is to be saved in a work file. The FSAVE routine calls the OPNFIL subroutine which opens the work file. The integer flags CRTFLG and EDFLG determine what file extension or identifier will be used to identify the file as a unit, block, or superblock work file. This extension or identifier is set by the subroutine OPNFIL. The value of the extension or identifier is then used to determine which specific saving routine will be used for saving the information. The READIN module is the counterpart of the FSAVE module for reading in previously saved unit, block, or simulation information. READIN also calls OPNFIL and is used to read in unit, block, or simulation work files for editing or for insertion or replacement operations.

The OPNFIL module controls all work file opening procedures. This includes obtaining filenames, checking for illegal filenames, determining filename modifiers or extensions to identify the three types of work files, and the creation of backup files. The IRDFLG variable is equal to one if a workfile is to be read, and is not equal to one if a work file is to be written. This subroutine will probably require customizing for specific computers. Parameter statements have been included to ease customization.

RDUNT, RDBLK, and RDSIM are called by the READIN module and read in the unit, block and simulation information, respectively. There is no read superblock module. SAVUNT, SAVBLK, and SAVSIM are called by the FSAVE module to save unit, block, and simulation information in work files. SAVBLK is also called by the edit(insert/delete/save) module (HVACGEN6) to save block information from simulations. The subroutine SAVSUP is not currently used but is intended to allow the saving of superblock information. The TYPEIN subroutine is used throughout HVACGEN to access the information about types in the TYPES data file on logical unit LUTYPR. This routine allows the choice of direct or sequential file access to the data file. 


\subsubsection{HVACGEN4 - view module}

The view module contains subroutines to display the contents of work files. The VIEW subroutine provides the view command menu to VIEW units, blocks, and simulations. Depending on the choice, this routine then calls VEWUNT, VEWBLK, or VEWSIM to view the particular information requested. VEWUNT gives the inputs, outputs and parameters for a unit. VEWBLK calls VEWUNT repeatedly to give information on the units in a block. VEWSIM calls several routines to show the information on the simulation. STRUCT is called by VEWSIM to show the structure of the simulation. STRUCT gives an overall view and calls VEWUNT to shown the information on selected specific units in the simulation. VARVAL is called by VEWSIM to display information on the initial values of the simulation variables. BOUND is called by VEWSIM to display information on the boundary variables. RPTVAR is called by VEWSIM to display information on reported variables. ERROR is called by VEWSIM to show the error tolerances and the variable scan and freeze options. REVEW is used to determine if reserved words have been typed in at any time. Reserved words allowed are ABort and HElp. Subroutine VEWALL is called to produce a complete description of a simulation work file. VEWALL calls most of the other routines in the view module.

\subsubsection{HVACGEN5 - edit (changes) module}

The edit (changes) module contains subroutines to edit the information in work files for cases where units and blocks are not inserted, deleted, replaced or saved. The EDIT subroutine displays the EDIT command menu and allows the selection of unit or simulation editing. Block and superblock editing are not supported. EDIT calls either EDUNT to edit a unit or EDSIM to edit a simulation. EDUNT allows the editing of unit inputs, outputs, and parameters.

EDSIM is the simulation work file information editing routine. It calls a number of secondary routines to edit parts of the simulation information. A menu is displayed and the choice is evaluated to determine which of the editing routines to call. EDTITL is called by EDSIM to allow editing of the title of the subroutine. EDSTR is called by EDSIM to allow editing of any of any of the units in the simulation. EDSTR calls STRUCT from the view modules and then EDUNT. EDVAL is called by EDSIM to allow editing of the initial variable values. EDBND is called by EDSIM to allow editing of simulation boundary variables. EDBND calls either INSERT or DELETE. INSERT allows adding new boundary variables. DELETE allows the removal of boundary variables and is very similar to INSERT. EDREP is called by EDSIM and allows the reported variables to be edited. Like EDBND, EDREP calls subroutines for changing reporting intervals or for inserting or deleting reported variables: RPCHNG, RPINRT, and RPDELT. RPCHNG allows the changing or a reporting interval. RPINRT allows the insertion of new reported variables and is similar to INSERT. RPDELT allows the deletion of reported variables and is similar to DELETE. EDERR is called by EDSIM to edit the error tolerances and variable scan and freeze options.

REEDT is the reserved word module for the edit module and allows help or abort 
commands to be entered at any time during editing.

\subsubsection{HVACGEN 6 - edit (insert/delete/save) and help module}

This module contains subroutines used to perform the insertions, deletions, replacement, or saving of blocks and/or units. A help subroutine is also located in this module.

The Subroutine INSSIM is called by EDSIM (HVACGEN5) and allows the insertion of a unit or block into a simulation. This routine uses a menu to determine what insertion action is to be taken and calls the appropriate subroutine, INSUNT, INSBLK, or REPSIM. INSUNT is called by INSSIM to insert a unit. A block number is requested and the option to read from a file or create from the console is determined. If the insertion is from a file, RDUNT (files module) is called. All unit number arrays and variable index arrays are shifted over starting at a spot which depends on the block number. The resulting hole is filled by the new unit information. If the insertion is from the console, the create unit routine (CRUNIT)is called. The highest unit number is determined and the number after this is assigned to the new unit. Subroutine INSCHK is called by INSSIM to find the superblock number and the last unit number in the block selected for unit insertion.

INSBLK is called from INSSIM to insert a block into the simulation from a file or the console. The number of units in the block must be known and the arrays in the simulation are shifted by this many spaces. The new unit information for all the units in the block is then inserted. INCK2 is called from INSBLK to determine superblock and unit information.

Subroutine RECALC is called whenever a unit or block is inserted. If any units have been added this routine checks to see if there are any new indicies that are higher than existing indices and shifts the state vector (initial value array) over the appropriate amount so that there are locations for the new variables. Subroutine TYPINF is a support routine for RECALC which calls TYPEIN to determine the numbers of variables for specific types.

Subroutine REBND recalculates the position of boundary variables in the state vector after insertion or deletion of a unit. REREPT provides the same function for the reported variables.

DELSIM is called from EDSIM to delete parts of a simulation. It is set up very much like INSSIM. Subroutine DELUNT is called to delete a unit. Subroutine DELBLK is used to delete a block. To delete a unit or block, the arrays in the program are shifted over and maximum counters are recalculated. DELCHK and DELCK2 are used by DELSIM and DELBLK just as INSCHK and INSCK 2 are used by INSSIM and INSBLK.

REPSIM is called by INSSIM and is used for replace operations. REPUNT is used to replace a unit. The information for an existing unit is overwritten by this routine.

Subroutine EXTBLK is called from EDSIM (HVACGEN4) whenever the SAVE command is 
used to allow block information to be saved in a block work file. EXTBLK calls SAVSIM (HVACGEN3) to write information into a file.

Use of the HELP command causes the HELP subroutine to be called. HELP is currently a simple subroutine which generates a primitive listing of program inf ormation.

\subsection{Description of SLIMCON subroutines}

The SLIMCON program consists of a main program and six subroutines. The main program is large and contains most of the FORTRAN code. The program basically acts as a 'filter,' reading in the work file, making calculations and writing to the model definition file. Subroutine FILEOP gets the work file name from the user and opens the work file and the model definition file. The TYPAR subroutine is used to access the types information file. As information is read or calculations are made, reports on configuration parameters are sent to the console using subroutine REPORT. REPORT determines if the parameters are close or exceeding the maximum values and issues warnings or error messages. Subroutines VARCHK and OUTCHK are also used for error checking. VARCHK is used to determine if any of the variables declared as time-dependent boundary conditions are also solved for simultaneously. If this is true a warning is issued. OUTCHK issues a warning if any simulation variable is an output of two or more components. Subroutine TDBVIS finds the largest number of timedependent boundary variables in any one superblock.

\subsection{Increasing or decreasing the simulation size capability}

The maximum size of the system that HVACSIM can simulate is determined by the dimensions of arrays and by parameters in the source code of the HVACSIM package programs. It is possible to increase the size of the system that can be simulated to allow more complex simulations, or to decrease the size of the system that can be simulated in order to decrease the size of the programs. This is not a trivial change, but is relatively straightforward since all changes that must be made to change the simulation size capability are in FORTRAN parameter statements. There are approximately 21 parameters that determine the maximum size capability. The PARAMETER statements that contain these parameters are grouped together but are duplicated in every subroutine in the three HVACSIM programs which uses any of the parameter values. This means that to change one parameter, all instances of the PARAMETER statement for the parameter to be changed must be altered in all the source code in the HVACGEN package. This can be easily accomplished using an editor with a search and replace capability. The parameters available and the maximum values as the package is distributed are listed section 3.10 . 


\section{EXAMPLES OF MODEL DEVELOPMENT, CONFIGURATION, AND EXECUTION}

This chapter presents a number of examples of system simulations using HVACSIM $^{+}$. The examples are arranged in order of increasing complexity, using the editing features of HVACGEN to combine simple systems into more complicated ones.

The examples are intended to serve several purposes: to illustrate some of the features of HVACGEN and the use of HVACSIM, to allow verification that HVACSIM $^{+}$is operating correctly after installation on a new system or after any modifications, and to serve as a tutorial in the design, creation, execution, and (to a limited extent) debugging of system simulations using HVACSIM $^{+}$. The prospective user is encouraged to familiarize himself with the examples before proceeding to develop his own simulations.

The TYPES mentioned in this chapter are summarized in the Appendix. More complete descriptions of the component models can be found in the HVACSIM ${ }^{+}$ reference manual [1]. The use of the building loads model involves some special considerations, and is not covered in these examples. For an example of the building model, the reader is referred to reference [2].

\subsection{Example 1: Pipe and Valve}

\section{1 .1 Designing a Simulation}

This example consists of two UNITS: the TYPE 3 inlet pipe and the TYPE 9 valve. The system is represented schematically in Figure 6.1-1. Ten state variables are identified in the figure: three pressures, one flow rate, three temperatures, and three control signals. Parameter values are indicated beneath the sketch of the system. Definitions of the parameters, and identification of the state variables as inputs and outputs, can be found in the appendix or in reference [1].

Determining appropriate parameter values for the components of a simulation can be a difficult and time-consuming task. An understanding of factors influencing the design of a system is helpful at this stage.

Referring to the HVACSIM ${ }^{+}$Reference Manual [1], Section 4.0, the authority of a valve can be expressed as the flow resistance of the open valve divided by the total flow resistance across the system. Using the flow resistance parameter values shown in Figure 6.1-1, the valve authority in this simulation is given by

$$
a=\frac{\mathrm{K}_{\text {valve }}}{\mathrm{K}_{\text {valve }}+\mathrm{K}_{\text {pipe }}}=\frac{1.5}{13.5+1.5}=0.10
$$

or $10 \%$. The desired maximum flow rate, when the valve is open, determines the difference between the inlet and outlet pressures, P1 and P3:

$$
\mathrm{P} 1-\mathrm{P} 3=\left(\mathrm{K}_{\text {valve }}+\mathrm{K}_{\mathrm{pipe}}\right) \mathrm{W}^{2}=15 \mathrm{~W}^{2}
$$


where $W$ is the mass flow rate. Thus if a maximum flow rate of $1.5 \mathrm{~kg} / \mathrm{s}$ is desired, P1 and P3 should be assigned values such that Pl minus P3 equals 33.75 .

The minimum (leakage) flow rate, when the valve is closed, can be expressed as a function of the leakage parameter, L, and the valve authority, a. To a good approximation, the relative leakage rate is given by

$$
\frac{W_{\text {min }}}{W_{\text {max }}}=L a^{-0.5}
$$

The value of $L$ in this simulation was chosen to yield a leakage flow ratio of 0.01 , or a turn-down ratio of 100 .

The transport delay in the pipe, $T_{x}$, is given by

$$
\mathrm{I}_{\mathrm{x}}=\mathrm{D} \operatorname{Vol} / \mathrm{W}_{1}
$$

where D is the density of the fluid. At a flow rate of $1.5 \mathrm{~kg} / \mathrm{s}$, the transport delay is ten seconds.

Figure 6.1-2 is an "Information Flow Diagram" showing the manner in which the two components are linked together. The components are represented by boxes with inputs, outputs, and parameters. Connections between components are formed by giving an input of one component the same state variable index number as the output of another component. For example, pressure 22 is an output of Unit 2 and an input of Unit 1.

Any state variable which is the dependent variable in a differential equation or which is an output of one unit and also an input to one or more other units will be solved simultaneously by the main simulation program. This statement involves a subtle and somewhat confusing distinction: in cases where an output of a unit is also an input to the same unit, the status of the variable cannot be determined at a glance. Two examples of variables which fit this description are the control variable $\mathrm{C} 2$ and the temperature T2. The control variable C2 is solved simultaneously because of its involvement in a differential equation. If the actuator time constant (parameter 2 of TYPE 9) were changed to one second or less, however, C2 would be determined within the TYPE 9 subroutine and would cease to be a simultaneously solved variable. On the other hand, the temperature $\mathrm{T} 2$ is solved within the TYPE 3 subroutine in this simulation and is not considered a simultaneous variable. If the seventh parameter of the pipe model, MODE, were made negative, the differential equation involving $\mathrm{T} 2$ would be solved by the equation solver and $\mathrm{T} 2$ would become a simultaneous variable. Thus the number of simultaneous equations and the number of differential equations in a simulation may depend on parameter values. In this simulation there are three simultaneously solved variables: $\mathrm{P} 2$, W1, and $\mathrm{C} 2$.

State variable C3 is an output but not an input, so it is not solved simultaneously. Its sole purpose is to provide the user with information about the state of the system. T2 is also considered an informative output in this system. 
The remaining variables are inputs but not outputs, and serve as boundary conditions. Boundary variables may be either constant (time independent) or time dependent. In this example, the inlet and outlet pressures P1 and P3 and the ambient temperature $\mathrm{T} 3$ will be constant boundary conditions, retaining their initial values throughout the simulation. When HVACGEN is run to set up the simulation, the requested valve position $\mathrm{Cl}$ and the inlet temperature $\mathrm{Tl}$ will be declared time dependent, meaning that their values as functions of time are to be read from a file during the course of the simulation. Details of this procedure will be discussed below.

\subsubsection{Creating a Simulation}

Listing 6.1-1 is a record of the interactive HVACGEN session in which the simulation description was created. First HVACGEN is requested to create a simulation. Note that the command CReate SImulation could have been entered on a single 1 ine. Next HVACGEN is told to include a TYPE 3 component in the simulation. HVACGEN then prompts the user for state variable index numbers for each input and output. At this stage a system schematic such as Figure 6.1-1 is indispensable. An information flow diagram such as Figure 6.1-2 may also be helpful. Parameter values are then entered, completing the description of the first UNIT in the simulation. The same procedure is repeated for the TYPE 9 component, which becomes the second UNIT.

Next HVACGEN asks for a title for the simulation, and then allows the user to approve or modify the default error tolerances. The default values are somewhat arbitrary, and experimentation is encouraged. Briefly, RTOLX and ATOLX are relative and absolute error tolerances, respectively, used primarily to decide when variables should be frozen or unfrozen. In this example RTOLX has been changed to 0.005 , meaning that relative errors of half a percent are acceptable. XTOL is an error tolerance used only by the equation solver. The recommended value is the square root of the machine precision, although a smaller value may sometimes be necessary for satisfactory accuracy. TTIME is a time interval associated with the integration of differential equations. A large value of TTIME results in greater accuracy and shorter time steps. The default value of one second yields satisfactory results in simple simulations, but larger values are likely to be necessary in more complicated simulations. A value of ten seconds has been chosen for this example.

HVACGEN then summarizes the number of variables in each category, and asks for an initial value of each variable. It is important to recognize that variables which are constant boundary conditions will retain their initial values throughout the simulation, and variables which are solved simultaneously will not necessarily retain their initial values. For example, in this simulation flow is driven by the difference between the inlet and outlet pressures, P1 and P3. As discussed above, this pressure difference (together with the flow resistance parameters) determines the maximum flow rate through the system. The initial value of the flow rate wl provides a starting point for the equation solver, but ultimately has no effect on the calculated flow rate.

Next HVACGEN prompts the user to identify boundary variables which are time dependent. In this example the inlet temperature $\mathrm{Tl}$ and the requested valve position $\mathrm{Cl}$ have been identified as time dependent boundary variables. Note 
that HVACGEN will allow any variable in the simulation to be declared a time dependent boundary variable, even if the variable should actually be solved simultaneously. However, SLIMCON issues a warning message if anything other than a boundary variable has been declared time dependent.

A reporting interval and a 1 ist of reported variables are then entered. In large simulations containing more than one superblock, reporting intervals and reported variables may be different for each superblock. Finally, a variable freezing option and an input scan option must be selected. These options are discussed in section 3.6.3(2). Briefly, the low-numbered choices are fastest and the high-numbered modes are most accurate. The variable freezing option has no effect on simulations with only one block, and the input scan option has no effect on simulations with only one superblock. Thus in this example both options are irrelevant, and the simplest modes have been selected.

This completes the creation of the simulation description. HVACGEN requests a file name, writes the information defining the system to this file, and returns to the main menu. In the present example, the HVACGEN output "work file" is named EXAMPLEl, to which HVACGEN has added the extension ".SIM" to indicate that the file contains a simulation description.

Finally, the VIEW ALL command has been used to produce a summary of the simulation configuration at the end of Listing 6.1-1.

\subsubsection{Creating the Model Definition File}

Listing 6.1-2 is the simulation work file, EXAMPLEl.SIM, written by HVACGEN. As discussed in Section 3.10, this file serves as input to the utility program SLIMCON. Listing $6.1-3$ is the tabular output of SLIMCON summarizing the size of the simulation. The primary purpose of this output is to warn the user if any category of information exceeds the array dimensions in HVACSIM. Listing 6.1-4 is the model definition file, in this case EXAMPLEl.DAT, which is written by SLIMCON and serves as input to HVACSIM.

\subsubsection{Preparing the Boundary Value File}

Listing 6.1-5 is the boundary variable file prepared for use with this example. The first column contains values of time, beginning at zero. The remaining columns contain values of the boundary variables. Column 2 contains values of the first boundary variable identified to HVACGEN, in this case temperature Tl. Column 3 contains values of the second boundary variable identified to HVACGEN, in this case control signal Cl. These simulation inputs represent a thirty-second ramp change in the valve position from time 30 to time 60 seconds, and again from 150 to 180 seconds, followed by five degree step changes in the water inlet temperature at times 210 and 255 seconds.

Note the use of the "reset convention": repeated values of time at 30,60 , $150,180,210$, and 255 seconds. When the simulation is run, it will integrate up to these reset times, and then reduce the time step and the order of integration to their minimum values before proceeding. The boundary values are interpolated as necessary (using third order Lagrangian interpolation) up 
to the reset time, and then a new set of values are read in before proceeding, so that interpolation across a step change or around the corner of a discontinuous change in slope is avoided. The resets also improve the accuracy of the simulation by keeping the time step from overshooting abrupt changes in the boundary file.

\section{1 .5 Running a Simulation}

Listing 6.1-6 is a record of the procedure for starting a simulation using HVACSIM. The program begins by asking for minimum and maximum time step sizes and the simulation stopping time. Next it asks if an Initial state file is to be read. This option has not been selected here, but will be used and discussed in later examples. Then the program asks whether the "Building shell Model" (consisting of types 50, 51, and 52) is used in this simulation. The default answer, no, is chosen in all examples in this chapter. Use of the building shell model is discussed in reference [2].

As stated in Section 5.2.1, SUBROUTINE OPNFIL defines default names for all input and output files used by HVACSIM, and associates each file with a logical unit number assigned in the BLOCK DATA routine. When HVACSIM is run, the user is given the choice of using the default names for all files or entering alternative names for any or all files. In this example default names have been used for all files except the model definition file, for which the name EXAMPLE1.DAT has been specified.

HVACSIM includes several options for writing diagnostic information to the output file with the default name MODSUM.DAT. This information pertains to the freezing and unfreezing of variables and to the progress of SNSQ, the simultaneous equation-solving portion of the main program. The option has not been used in this example, though information on the available choices has been elicited. When mode $1,2,3$, or 4 is selected, HVACSIM prompts the user for starting and stopping times for the diagnostics.

HVACSIM also allows the progress of the simulation to be followed on the user's console (or other output device). This option has been selected, and the resulting output is included in Listing 6.1-6. Here TIME is the current simulation time in seconds, NTIME is the number of time steps taken, TSTEP is the interval since the previous simulation time, and PSTEP is a preliminary estimate of the interval until the next simulation time. It is also possible to have the values of up to five variables reported to the console as the simulation progresses. This option has not been selected here.

\subsubsection{Discussion of Simulation Results}

The time step algorithm is quite sensitive to such factors as machine precision. The precise times and step sizes shown in Listing 6.1-6 are unlikely to be duplicated exactly when the program is run on a different computer.

HVACSIM produces three files on output. INITOUT.DAT is a file of numbers defining the state of the system at the simulation stopping time, and may be used as the initial state of subsequent simulation runs. This file is reproduced in Listing 6.1-7 for the sake of completeness. MODSUM.DAT, shown in 
Listing 6.1-8, contains a description of the simulation configuration, and interpolated values of the reported variables at equal time intervals. MODOUT.DAT, shown in Listing 6.1-9, contains values of the same reported variables at the actual simulation times. This file is suitable for any further processing of the simulation results which may be required.

Two graphs of results from this example are presented after the listings, to point out certain features of HVACSIM. Note, however, that the software used to generate the graphs is net included in the HVACSIM ${ }^{+}$package.

The solid lines in figure 6.1-3 show the mass flow rate through the valve as a function of the actual valve position, C3. Although the TYPE 9 valve is nominally linear, the actual characteristic depends on the authority of the valve over the system in which it is installed, just as a real valve's installed characteristic differs from its nominal characteristic. Two solid lines are graphed: one as the valve closes, and one as it opens. The difference between these 1 ines is due to numerical error in the computation.

The dashed lines in figure 6.1-3 are the mass flow rate through the valve as a function of the actuator position, C2, which differs from C3 due to the valve hysteresis parameter of 0.2 specified in this simulation. The hysteresis effect illustrated here represents mechanical slack in the linkage between the actuator and the valve.

The inlet and outlet temperatures $\mathrm{T} 1$ and $\mathrm{T} 2$ are plotted in Figure 6.1-4. During the first 25 seconds of the simulation, the outlet temperature changes from its somewhat arbitrary initial value of $80 \mathrm{C}$ to its steady state value of $78.4 \mathrm{C}$. The first valley in the curve is the response to the changes in flow rate. The response time is slower when the flow rate is decreased than when it is increased because the time constant used in the pipe model is inversely proportional to the flow rate and because the transport time is longer. The second valley is the response to inlet temperature step changes, shown as a dotted line. The transport delay is clearly visible here, as is the effect of the pipe's thermal capacitance. 


\section{System Schematic}

$\left(T_{3}=\right.$ ambient $)$

Unit 1

Type 3

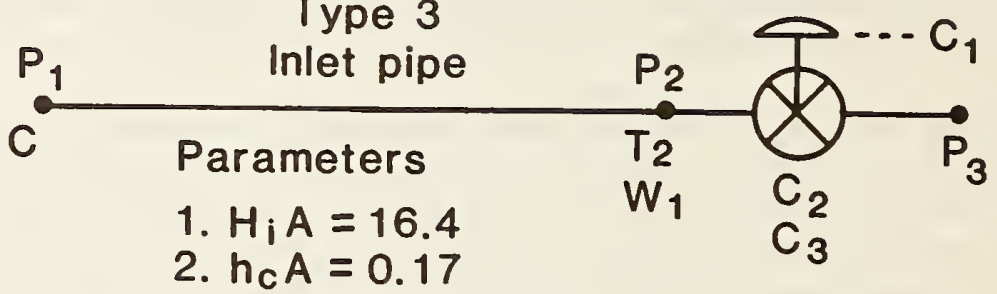

2. $h_{c} A=0.17$

3. $C_{m}=25.2$

Parameters

4. $\mathrm{Vol}=0.015$

1. $K=1.5$

5. $K=13.5$

6. $\mathrm{H}=0$

2. $T=5 \mathrm{sec}$

7. Mode $=2$

3. $L=0.00316$

4. Hys $=0.2$

Figure 6.1-1. Example 1: System schematic diagram

2. Information Flow Diagram

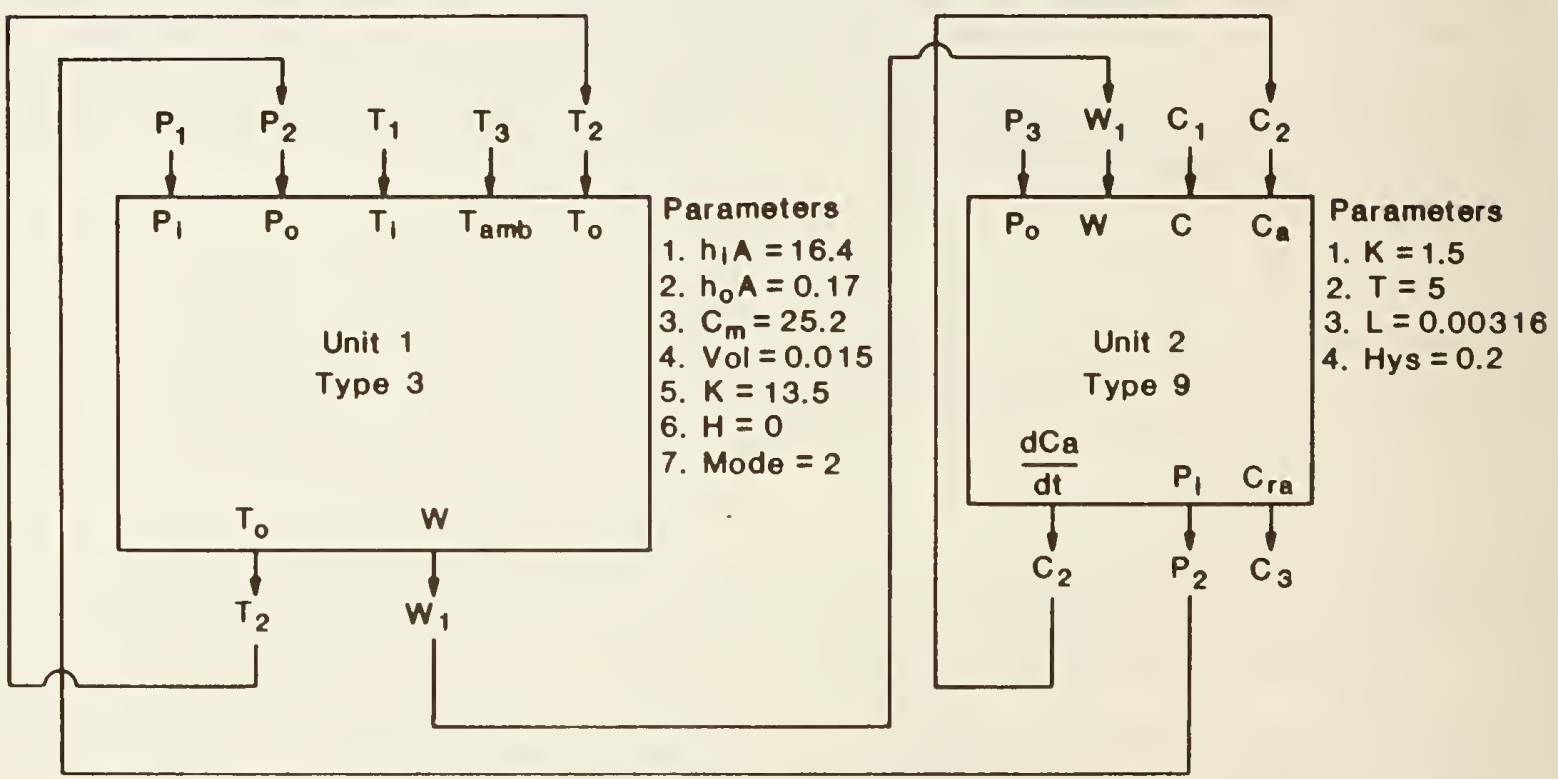

Higure u.1-\%. Fxample 1: Information flow diagram 
Listing 6.1-1. Interactive HVACGEN session to create simulation EXAMPLEl. Note: user responses are preceded by the following prompt: >

HVACGEN - Simulation GENeration Program

Version $1.7(04-02$ 1985)

Choose from the list below:

CReate (sImulation, BLock, UNit)

EDit (SImulation, UNit)

VIew (sImulation, BLock,UNit)

HElP

ENd

Selection?

$>$ CREATE

Create a:

SImulation

BLock

UNit

$>$ SIM

Superblock number $=1$

BLock number $=1$

Enter the type number (or TYPES for list of types)

$>3$

Initializing Types Information...

INLET CONDUIT (DUCT OR PIPE)

IN PUTS

index for INLET FLUID PRESSURE

PRESSURE

$>1$

index for OUTLET FLUID PRESSURE

PRESSURE

$>2$

index for INLET FLUID TEMPERATURE

TEMPERATURE

$>1$

index for AMBIENT AIR TEMPERATURE 


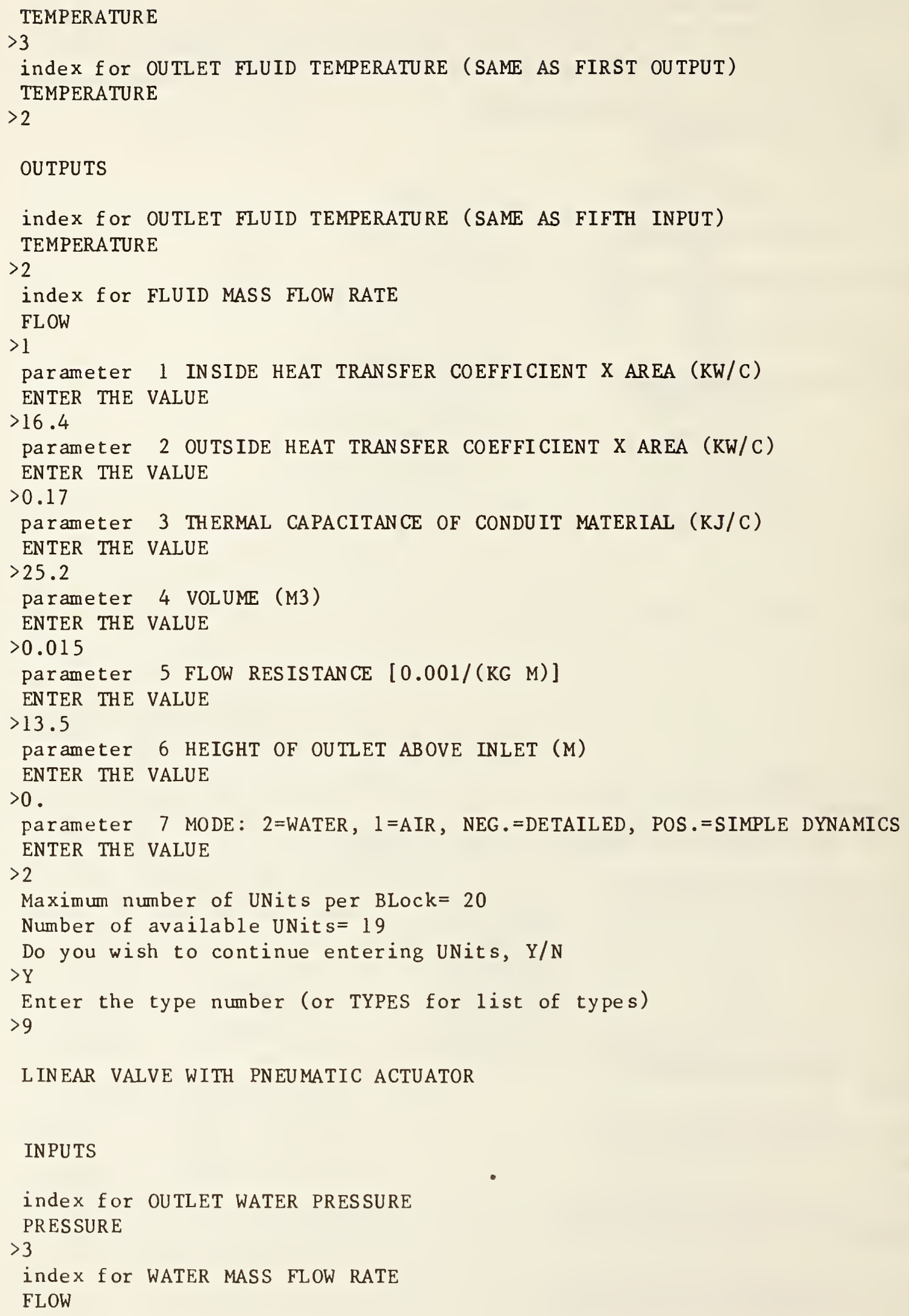




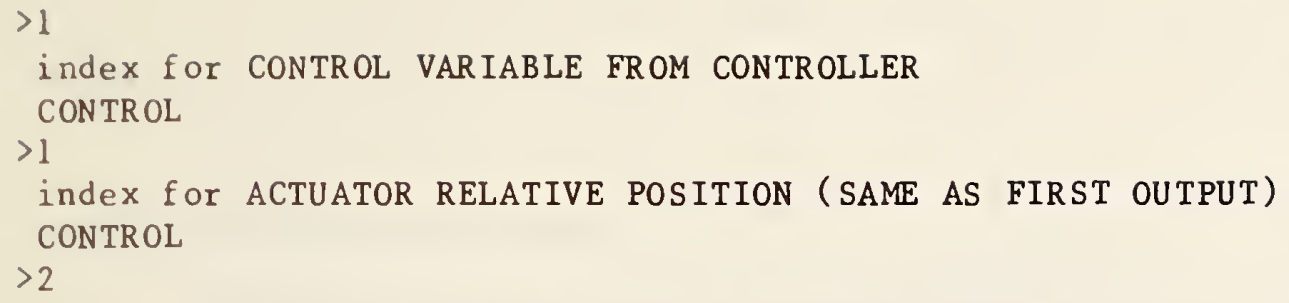




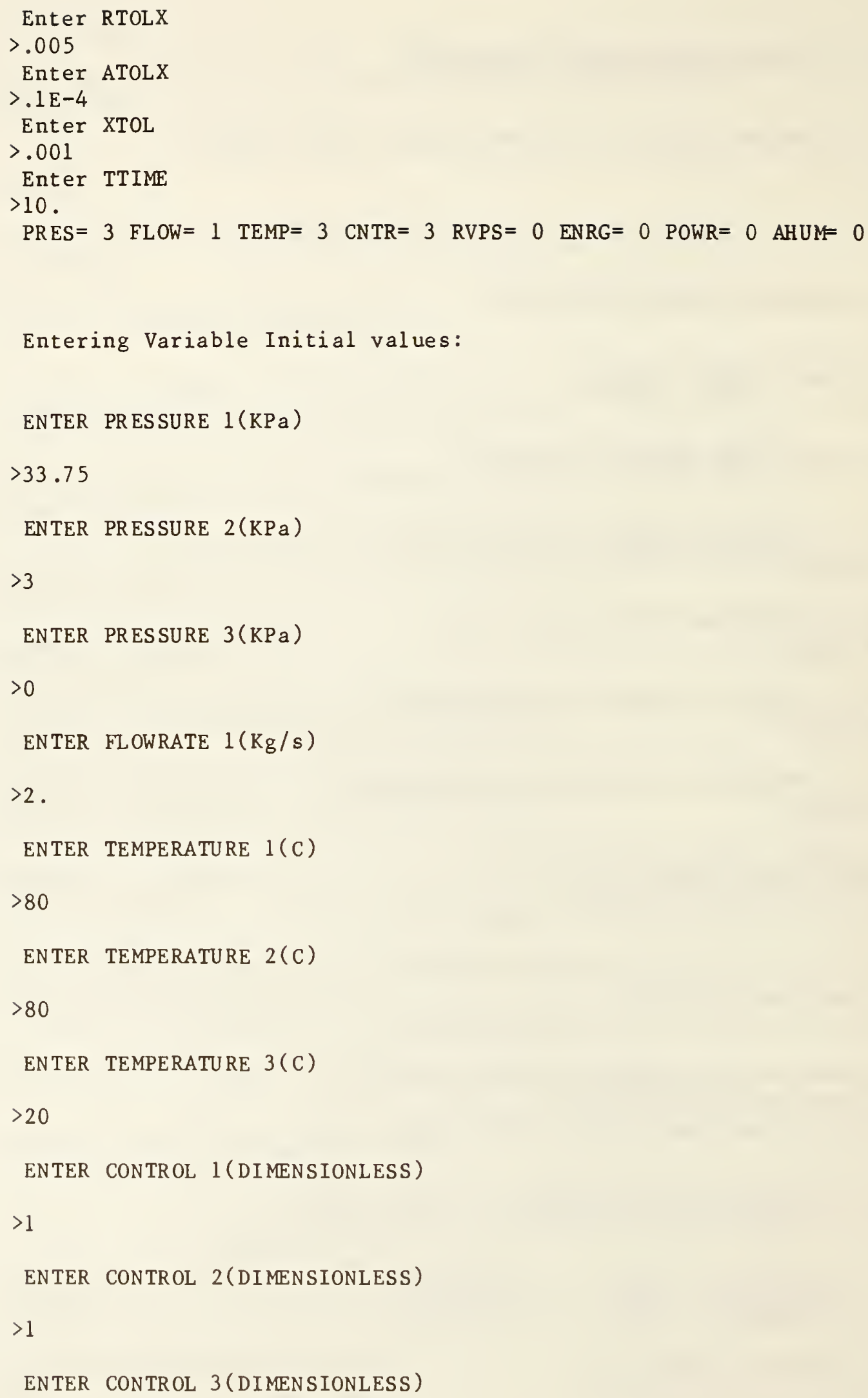


Entering Boundary Variables:

Enter a PRESSURE boundary variable or CR to move on $>$ Enter a FLOW boundary variable or $C R$ to move on $>$ Enter a TEMPERATURE boundary variable or CR to move on $>1$

Enter a TEMPERATURE boundary variable or $C R$ to move on $>$ Enter a CONTROL boundary variable or CR to move on $>1$ Enter a CONTROL boundary variable or CR to move on $>$

Entering Reported Variables for Superblock 1

Enter the reporting interval for this SUperblock in seconds $>10$

Enter a PRESSURE reported variable or $C R$ to move on $>2$

Enter a PRESSURE reported variable or $C R$ to move on $>$

Enter a FLOW reported variable or $C R$ to move on $>1$

Enter a TEMPERATURE reported variable or $C R$ to move on $>1$

Enter a TEMPERATURE reported variable or $C R$ to move on $>2$

Enter a TEMPERATURE reported variable or $C R$ to move on $>$

Enter a CONTROL reported variable or $C R$ to move on $>1$

Enter a CONTROL reported variable or CR to move on $>2$

Enter a CONTROL reported variable or $C R$ to move on $>3$

Enter the variable freezing option for this SUperblock: 0,1 , or 2 . $>0$

Enter the variable input scan option for this SUperblock: 0 or 1 . $>0$

Enter the filename (Maximum of 8 characters)

$>$ EXAMPLEl

saving to work file.... 
HVACGEN - Simulation GENeration Program

Version 1.7 (04-02 1985)

Choose from the list below:

CReate (SImulation, BLock, UNit)

EDit (s Imulation, UNit)

VIew (SImulation, BLock, UNit)

HElP

ENd

Selection?

$>$ VIEW SIM

Enter the filename (Maximum of 8 characters)

$>$ EXAMPLE1

reading from work file...

What part of the simulation would you like to view:

ALl the simulation information (for documentation)

STructure (superblock, block, and unit Information)

VAriable initial values

ERror tolerances, variable scan and freeze options

BOundary variables

REported variables

Continue with the previous menu

$>$ ALL

EXAMPLE 1: INLET PIPE AND (NOMINALLY) LINEAR VALVE SU PERBLOCK 1

BLOCK 1

UNIT 1 TYPE 3 - INLET CONDUIT (DUCT OR PIPE)

UNIT 2 TYPE 9 - LINEAR VALVE WITH PNEUMATIC ACTUATOR 
$\begin{array}{llll}\text { UNIT } 1 & \text { TYPE } 3\end{array}$

INLET CONDUIT (DUCT OR PIPE)

1 INPUTS :

$\begin{array}{ll}\text { PRESSURE } & 1 \text { - INLET FLUID PRESSURE } \\ \text { PRESSURE } & 2 \text { - OUTLET FLUID PRESSURE } \\ \text { TEMPERATURE } & 1 \text { - INLET FLUID TEMPERATURE } \\ \text { TEMPERATURE } & 3 \text { - AMBIENT AIR TEMPERATURE } \\ \text { TEMPERATURE } & 2 \text { - OUTLET FLUID TEMPERATURE (SAME AS FIRST OUTPUT) }\end{array}$

2 OUTPUTS :

TEMPERATURE 2 - OUTLET FLUID TEMPERATURE (SAME AS FIFTH INPUT)

FLOW

1 - FLUID MASS FLOW RATE

3 PARAMETERS :

16.4000 INSIDE HEAT TRANSFER COEFFICIENT X AREA (KW/C)

0.170000 OUTSIDE HEAT TRANSFER COEFFICIENT X AREA (KW/C)

25.2000 THERMAL CAPACITANCE OF CONDUIT MATERIAL ( $\mathrm{KJ} / \mathrm{C}$ )

$0.150000 E-01$ VOLUME (M3)

13.5000 FLOW RESISTANCE [0.001/(KG M)]

0.000000 HEIGHT OF OUTLET ABOVE INLET (M)

2.00000 MODE: 2=WATER, 1=AIR, NEG.=DETAILED, POS.=SIMPLE DYNAMI

UNIT 2 TYPE 9

LINEAR VALVE WITH PNEUMATIC ACTUATOR

1 INPUTS :

$\begin{array}{ll}\text { PRESSURE } & 3 \text { - OUTLET WATER PRESSURE } \\ \text { FLOW } & 1 \text { - WATER MASS FLOW RATE } \\ \text { CONTROL } & 1 \text { - CONTROL VARIABLE FROM CONTROLLER } \\ \text { CONTROL } & 2 \text { - ACTUATOR RELATIVE POSITION (SAME AS FIRST OUTPUT) }\end{array}$

2 OUTPUTS :

CONTROL 2 - ACTUATOR RELATIVE POSITION

PRESSURE 2 - INLET WATER PRESSURE

CONTROL 3 - VALVE STEM RELATIVE POSITION

3 PARAMETERS :
1.50000
FLOW RESISTANCE [0.001/(KG M)]
5.00000
ACTUATOR TIME CONSTANT (SEC)
$0.316000 \mathrm{E}-02$
LEAKAGE PARAME TER (DIMENSIONLESS)
0.200000
HYSTERES IS PARAMETER (DIMENSIONLESS)

Initial Variable Values:

$\begin{array}{llrl}\text { PRESSURE } & 1 \rightarrow & 33.7500 & (\mathrm{KPa}) \\ \text { PRESSURE } & 2 \rightarrow & 3.00000 & (\mathrm{KPa}) \\ \text { PRESSURE } & 3 \rightarrow & 0.000000 & (\mathrm{KPa}) \\ \text { FLOW } & 1 \rightarrow & 2.00000 & (\mathrm{Kg} / \mathrm{s}) \\ \text { TEMPERATURE } & 1 \rightarrow & 80.0000 & (\mathrm{C}) \\ \text { TEMPERATURE } & 2 \rightarrow & 80.0000 & (\mathrm{C})\end{array}$




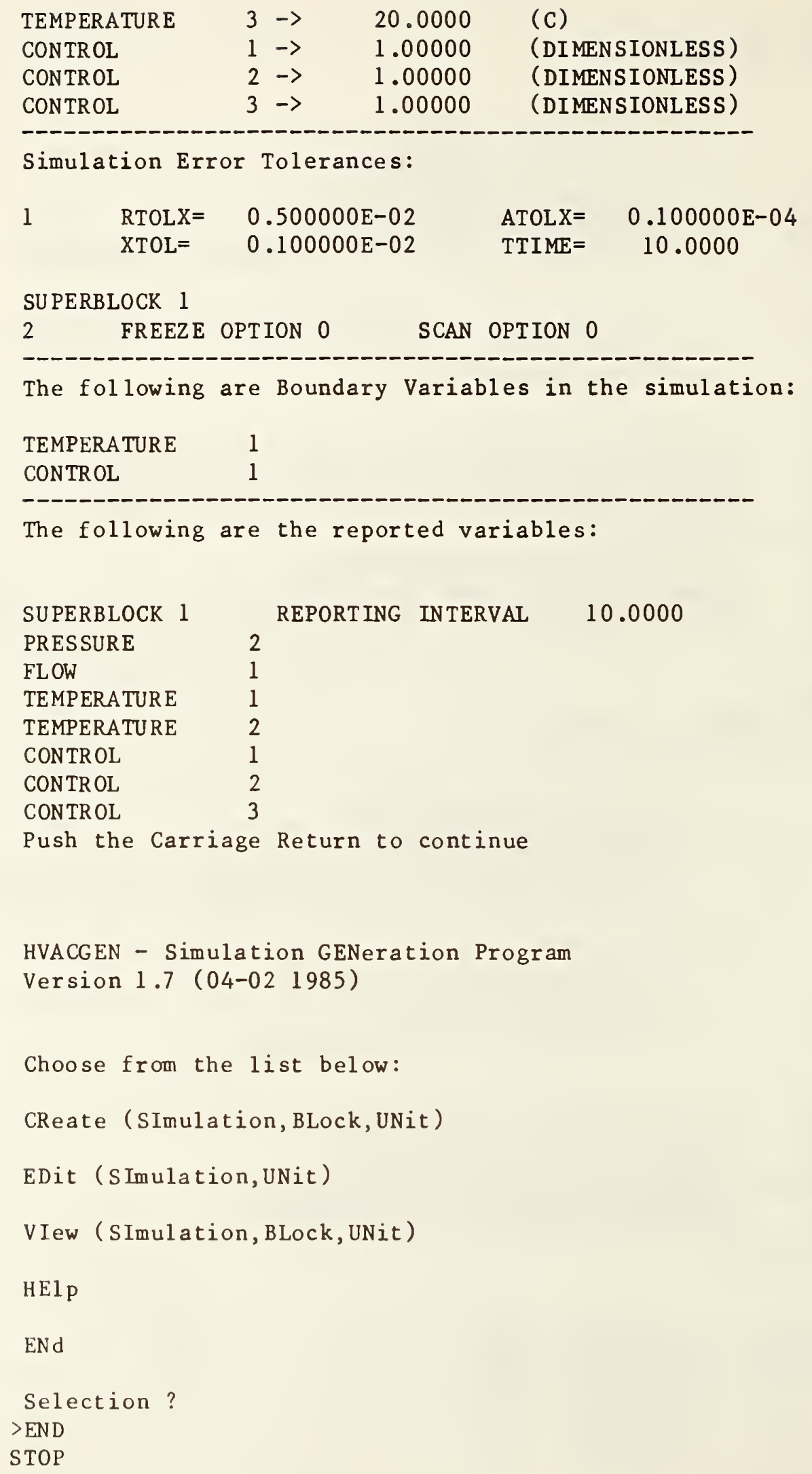


Listing6.1-2. EXAMPLEl.SIM: Simulation Work File, Output of HVACGEN, Input to SLIMCON

EXAMPLE 1: INLET PIPE AND (NOMINALLY) LINEAR VALVE

1

$0.500000 \mathrm{E}-0$

$0.100000 E-04$

$0.100000 \mathrm{E}-02$

$0.100000 E+02$

1

2

13

$\begin{array}{lllll}1 & 2 & 1 & 3 & 2\end{array}$

21

$0.164000 E+02$

$0.000000 E+00$

$0.170000 E+00$

$0.252000 E+02$

$0.150000 \mathrm{E}-01$

$0.135000 \mathrm{E}+02$

29

$\begin{array}{llll}3 & 1 & 1 & 2\end{array}$

223

$0.150000 \mathrm{E}+01$

$0.337500 E+02$

$0.200000 \mathrm{E}+01$

$0.100000 \mathrm{E}+02$

$0.800000 E+02$

$0.500000 \mathrm{E}+01$

$0.316000 \mathrm{E}-02$

$0.200000 \mathrm{E}+00$

$0.300000 \mathrm{E}+01$

$0.000000 \mathrm{E}+00$

$0.200000 \mathrm{E}+01$

$0.200000 E+02$

$0.100000 E+01$

$0.100000 \mathrm{E}+01$

$0.800000 E+02$

2

58

$70.100000 \mathrm{E}+02$

$\begin{array}{lllllll}2 & 4 & 5 & 6 & 8 & 9 & 10\end{array}$

$\begin{array}{lllllll}1 & 2 & 3 & 3 & 4 & 4 & 4\end{array}$

$\begin{array}{lllllll}2 & 1 & 1 & 2 & 1 & 2 & 3\end{array}$

0

0

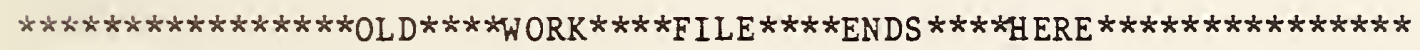

112

1

$\begin{array}{llllllll}3 & 1 & 3 & 3 & 0 & 0 & 0 & 0\end{array}$

10

7

7

7 
Listing $6.1-3$. SLIMCON Tabular Output

Simulation Work File to Model Definition File Converter

Version 2.1 (November 13, 1984)

Enter the simulation filename (Up to 8 characters) or carriage return to end. EXAMPLEl

1 superblocks in the simulation ......... MAXIMUM $=$

1 blocks in the simulation ...................

1 differential equations in the simulation MAXIMUM =

2 units in the simulation ................MAXIMUM $=200(1.0 \%$

2 units in a single block ............................

1 differential equations in one unit $\ldots .$. MAXIMUM $=10(10.0 \%$

5 inputs or outputs in a single unit ..... MAXIMUM $=20(25.0 \%$

7 parameters in a single unit ............MAXIMUM $=30(23.3 \%$

1 blocks in the largest superblock ........ MAXIMUM $=10(10.0 \%$

1 differential equations in one superblock MAXIMUM $=20(5.0 \%$

10 state variables in the simulation ....... MAXIMUM $=600(1.7 \%$

9 inputs or outputs in a single block .... MAXIMUM $=50(18.0 \%$

11 unit parameters in the simulation ....... MAXIMUM $=1000(1.1 \%$

3 simultaneous equations in a single block MAXIMUM $=30(10.0 \%$

0 simultaneous equations in one superblock MAXIMUM $=20(0.0 \%$

2 time dependent boundary variables $\ldots \ldots$ MAXIMUM $=30(6.7 \%$

2 boundary conditions in one superblock $\ldots$ MAXIMUM $=20(10.0 \%$

7 reported variables in one superblock $\ldots$ MAXIMUM $=30(23.3 \%$

Model Definition File Complete 
Listing 6.1-4. EXAMPLEl.DAT: Model Definition File, Output of SLIMCON, Input to HVACSIM

\section{EXAMPLE 1: INLET PIPE AND (NOMINALLY) LINEAR VALVE}

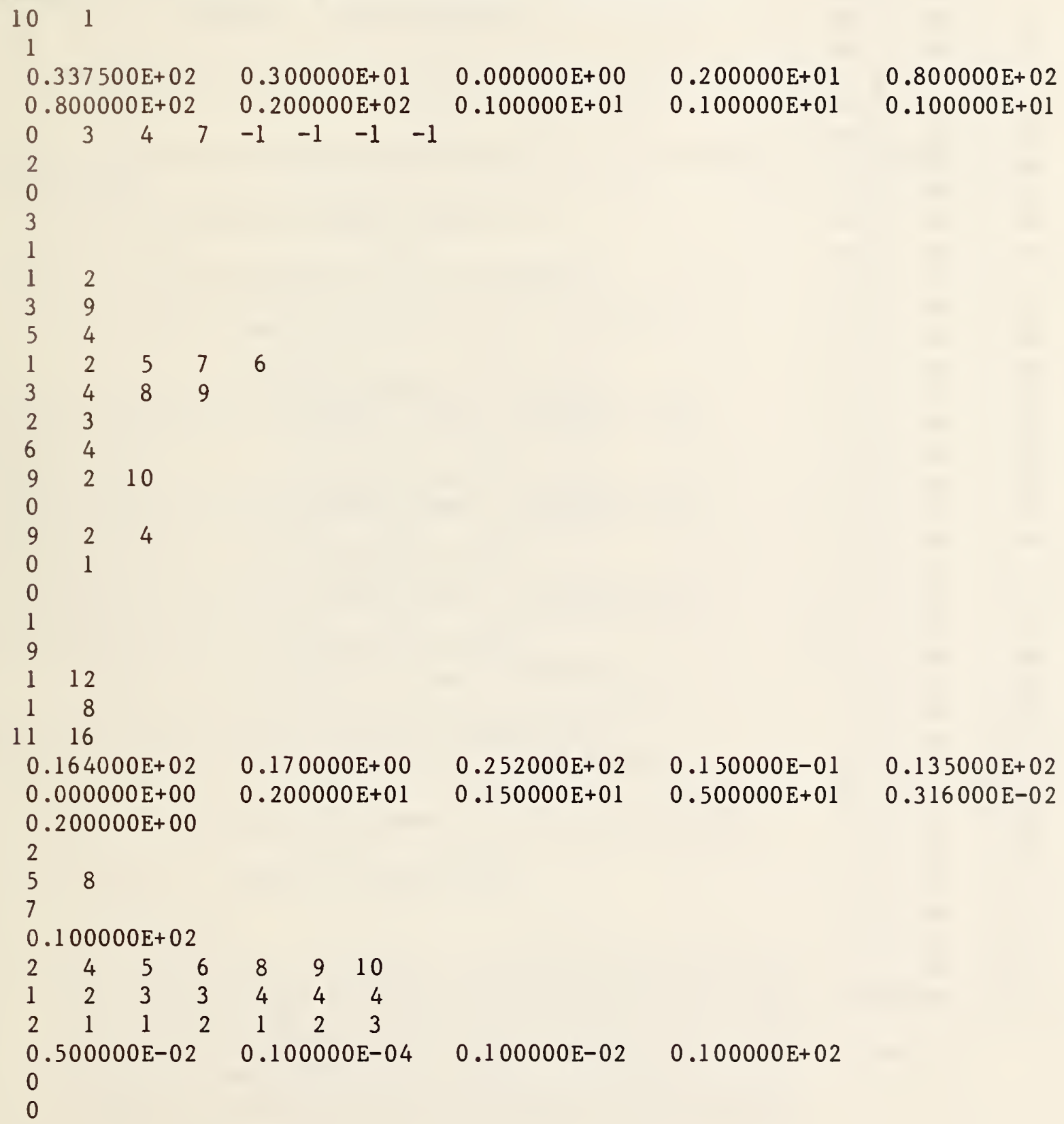




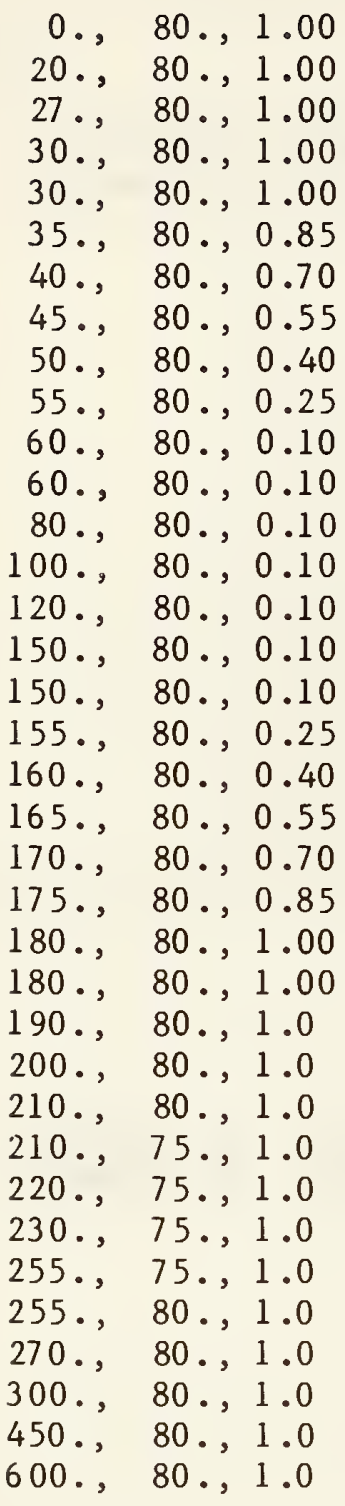




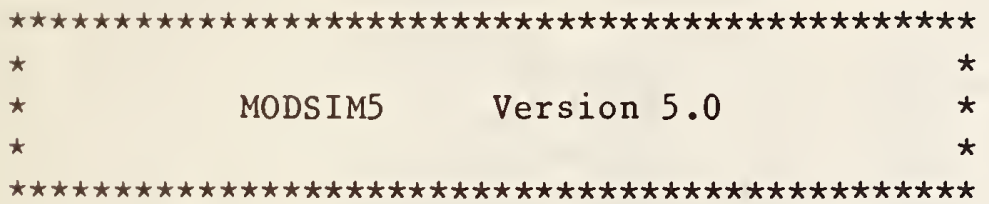

Enter MINIMUM TIME STEP, MAXIMUM TIME STEP, and SIMULATION STOPPING TIME: $0.1,60 ., 300$.

Is the Building Shell Model used? $<\mathrm{N}>$

Will the Initialization File be called? $\langle\mathrm{N}\rangle$

Use default file names for all files? (Y/N) $\langle\mathrm{Y}\rangle$ $\mathrm{N}$

Enter the name of the Model Definition File,

or Carriage Return for default name: MODELDEF.DAT

EXAMPLEl . DAT

Enter the name of the Boundary Variable File,

or Carriage Return for default name: BOUNDARY.DAT

EXAMPLE1 . BND

Enter the name of the Final state File,

or Carriage Return for default name: INITOUT.DAT

EXI IN IT.DAT

Enter the name of the Output Data File,

or Carriage Return for default name: MODOUT.DAT

Enter the name of the Simulation Summary File,

or Carriage Return for default name: MODSUM.DAT

Y

Do you want Diagnostic Information to be written $<\mathrm{N}>$ ?

Five Levels of detail are available:

0: No Diagnostics

1: Trace which state variables are solved simultaneously

2: Trace simultaneously solved state variables and their initial

and final values on each call to the equation solver

3: Trace simultaneously solved state variables and their estimated

values on every iteration within the equation solver

4: Perform the previous trace and dump Jacobian matrices

0

Enter $0,1,2,3$, or 4:

Y

Would you like to monitor the Simulation on Screen? $<N>$

Enter the INDEX NUMBER of the SUPERBLOCK to monitor

or zero (0) to monitor all superblocks. 
0

--During the simulation, up to five State Variables can be viewed-0

Enter the NUMBER of STATE VARIABLES to be viewed.

\begin{tabular}{|c|c|c|c|c|c|c|c|}
\hline $\mathrm{SB}$ & $1:$ & TIME & $=0.1$ & NTIME $=$ & $=1$ & TSTEP $=0.1$ & PSTEP $=0.2$ \\
\hline B B & $1:$ & TIME & $=0.3$ & NTIME $=$ & $=2$ & TSTEP $=0.2$ & PSTEP $=0.4$ \\
\hline$B$ & $1:$ & TIME & $=0.7$ & NTIME = & $=3$ & TSTEP $=0.4$ & PSTEP $=0.8$ \\
\hline$B$ & $1:$ & T IME & $=1.5$ & NTIME $=$ & $=4$ & TSTEP $=0.8$ & PSTEP $=1.6$ \\
\hline $\mathrm{SB}$ & $1:$ & TIME & $=3.1$ & NTIME $=$ & $=5$ & TSTEP $=1.6$ & PSTEP $=3.2$ \\
\hline SB & $1:$ & T IME & $=6.3$ & NTIME $=$ & $=6$ & TSTEP $=3.2$ & PS TEP $=6.4$ \\
\hline SB & $1:$ & TIME & $=12.7$ & NTIME & $=7$ & TSTEP $=6.4$ & PSTEP $=12.8$ \\
\hline SB & $1:$ & T IME & $=25.5$ & NTIME & $=8$ & TS TEP $=12.8$ & PSTEP $=25.6$ \\
\hline SB & $1:$ & TIME & $=30.0$ & NTIME & $=9$ & TSTEP $=4.5$ & PSTEP $=9.0$ \\
\hline SB & $1:$ & T IME & $=30.1$ & NTIME & $=10$ & TSTEP $=0.1$ & PSTEP $=0.2$ \\
\hline SB & $1:$ & TIME & $=30.3$ & NTIME & $=11$ & TSTEP $=0.2$ & PSTEP $=0.11129$ \\
\hline SB & $1:$ & T IME & $=30.4$ & NTIME & $=12$ & TSTEP $=0.1$ & PSTEP $=0.2$ \\
\hline SB & $1:$ & TIME & $=30.6$ & NTIME & $=13$ & TSTEP $=0.2$ & PSTEP $=0.282843$ \\
\hline SB & $1:$ & TIME & $=30.8$ & NTIME & $=14$ & TSTEP $=0.2$ & PS TEP $=0.159459$ \\
\hline SB & $1:$ & TIME & $=30.9$ & NT IME & $=15$ & TSTEP $=0.1$ & PSTEP $=0.1$ \\
\hline SB & $1:$ & TIME & $=31.0$ & NTIME & $=16$ & TSTEP $=0.1$ & PSTEP $=0.1$ \\
\hline SB & $1:$ & TIME & $=31.1$ & NTIME & $=17$ & TSTEP $=0.1$ & PSTEP $=0.1$ \\
\hline SB & $1:$ & T IME & $=31.2$ & NT IME & $=18$ & TSTEP $=0.1$ & PS TEP $=0.11$ \\
\hline SB & $1:$ & TIME & $=31.3$ & NTIME & $=19$ & TSTEP $=0.1$ & PSTEP $=0$. \\
\hline SB & $1:$ & T IME & $=31.4$ & NT IME & $=20$ & TSTEP $=0.1$ & PS TEP $=0.2$ \\
\hline SB & $1:$ & TIME & $=31.6$ & NTIME & $=21$ & TSTEP $=0.2$ & PSTEP $=0.236964$ \\
\hline SB & $1:$ & TIME & $=31.8$ & NTIME & $=22$ & TSTEP $=0.2$ & PS TEP $=0.992983$ \\
\hline SB & $1:$ & TIME & $=32.7$ & NTIME & $=23$ & TSTEP $=0.9$ & PSTEP $=1.25661$ \\
\hline SB & $1:$ & TIME & $=33.9$ & NTIME & $=24$ & TSTEP $=1.2$ & PSTEP $=1.4751$ \\
\hline SB & $1:$ & TIME & $=35.3$ & NTIME & $=25$ & TSTEP $=1.4$ & PSTEP $=1.6$ \\
\hline SB & $1:$ & T IME & $=36.9$ & NT IME & $=26$ & TSTEP $=1.6$ & PSTEP $=2.9$ \\
\hline SB & $1:$ & TIME & $=39.8$ & NT IME & $=27$ & TSTEP $=2.9$ & PSTEP $=3$. \\
\hline SB & $1:$ & TIME & $=43.1$ & NT IME & $=28$ & TSTEP $=3.3$ & PSTEP $=4$. \\
\hline SB & $1:$ & T IME & $=47.4$ & NTIME & $=29$ & TSTEP $=4.3$ & PSTEP $=3$. \\
\hline SB & $1:$ & T IME & $=51.1$ & NT IME & $=30$ & TS TEP $=3.7$ & PSTEP $=3.6$ \\
\hline SB & $1:$ & TIME & $=54.7$ & NT IME & $=31$ & TSTEP $=3.6$ & PSTEP $=4$. \\
\hline SB & $1:$ & TIME & $=59.5$ & NTIME & $=32$ & TS TEP $=4.8$ & PSTEP $=7.79888$ \\
\hline SB & 1: & TIME & $=60.0$ & NT IME & $=33$ & TSTEP $=0.5$ & PSTEP $=1.0$ \\
\hline SB & $1:$ & TIME & $=60.1$ & NT IME & $=34$ & TSTEP $=0.1$ & PS TEP $=0.1$ \\
\hline SB & $1:$ & T IME & $=60.2$ & NTIME & $=35$ & TSTEP $=0.1$ & PSTEP $=0.1$ \\
\hline SB & $1:$ & TIME & $=60.3$ & NT IME & $=36$ & TSTEP $=0.1$ & PSTEP $=0.58$ \\
\hline SB & 1: & TIME & $=60.8$ & NTIME & $=37$ & TSTEP $=0.5$ & PS TEP $=0.41$ \\
\hline SB & $1:$ & T IME & $=61.2$ & NTIME & $=38$ & TSTEP $=0.4$ & PS TEP $=0.8$ \\
\hline SB & $1:$ & TIME & $=62.0$ & NTIME & $=39$ & TSTEP $=0.8$ & PSTEP $=0.743616$ \\
\hline SB & 1: & TIME & $=62.7$ & NT IME & $=40$ & TSTEP $=0.7$ & PSTEP $=1.4$ \\
\hline SB & $1:$ & TIME & $=64.1$ & NTIME & $=41$ & TSTEP $=1.4$ & PSTEP $=1.85043$ \\
\hline SB & $1:$ & TIME & $=65.9$ & NTIME & $=42$ & TSTEP $=1.8$ & PSTEP $=2.56032$ \\
\hline \multirow{2}{*}{\multicolumn{3}{|c|}{$\begin{array}{l}\text { SU PERBLOCK } \\
\text { SUPERBLOCK }\end{array}$}} & \multirow{2}{*}{\multicolumn{2}{|c|}{$\begin{array}{l}\text { TIME STEP } \\
\text { TIME STEP }\end{array}$}} & \multicolumn{2}{|c|}{ REJECTED: PSTEP } & 0.6 \\
\hline & & & & & REJ & PS TEP & 0.1 \\
\hline SB & 1: & T IME & $=66.0$ & NT IME & $=43$ & TSTEP $=0.1$ & PSTEP $=0.1$ \\
\hline SB & $1:$ & T IME & $=66.1$ & NT IME & $=44$ & TSTEP $=0.1$ & PSTEP $=0.1$ \\
\hline SB & 1: & TIME & $=66.2$ & NT IME & $=45$ & TSTEP $=0.1$ & PSTEP $=0.780492$ \\
\hline
\end{tabular}




\begin{tabular}{|c|c|c|c|c|c|c|}
\hline SB & 1: & TIME & $=66.9$ & NTIME $=46$ & TSTEP $=0.7$ & PSTEP $=0.696695$ \\
\hline SB & 1: & T IME & $=67.5$ & NT IME $=47$ & TSTEP $=0.6$ & PS TEP $=1.2$ \\
\hline SE & 1: & TIME & $=68.4$ & NTIME $=48$ & TSTEP $=0.9$ & PSTEP $=1.01328$ \\
\hline $\mathrm{SF}$ & $1:$ & T IME & $=69.4$ & NTIME $=49$ & TSTEP $=1.0$ & PS TEP $=2.0$ \\
\hline SI & 1: & T IME & $=71 \cdot 3$ & NT IME $=50$ & TSTEP $=1.9$ & PSTEP $=2.0959$ \\
\hline SB & $1:$ & T IME & $=73.3$ & NTIME $=51$ & TSTEP $=2.0$ & PSTEP $=3.15911$ \\
\hline SB & $1:$ & TIME & $=76.4$ & NTIME $=52$ & TSTEP $=3.1$ & PSTEP $=2.45875$ \\
\hline $\mathrm{SB}$ & 1: & T IME & $=78.8$ & NTIME $=53$ & TSTEP $=2.4$ & PS TEP $=2.27548$ \\
\hline SB & 1: & TIME & $=81.0$ & NTIME $=54$ & TSTEP $=2.2$ & PSTEP $=3.46794$ \\
\hline 5$]$ & 1: & T IME & $=84.4$ & NTIME $=55$ & TSTEP $=3.4$ & PSTEP $=3.99716$ \\
\hline $\mathrm{SB}$ & 1: & TIME & $=88.3$ & NTIME $=56$ & TSTEP $=3.9$ & PSTEP $=4.54725$ \\
\hline & 1: & TIME & $=92.8$ & NTIME $=57$ & TSTEP $=4.5$ & PSTEP $=5.35143$ \\
\hline & $\mathrm{PEF}$ & BLOCK & 1: TIME & E STEP REJEC & TED: PSTEP = & 1.3 \\
\hline $\mathrm{SB}$ & $1:$ & T IME & $=94.1$ & NTIME $=58$ & TS TEP $=1.3$ & PSTEP $=0.687171$ \\
\hline SB & $1:$ & T IME & $=94.7$ & NTIME $=59$ & TSTEP $=0.6$ & PSTEP $=1.2$ \\
\hline SB & $1:$ & T IME & $=95.9$ & NTIME $=60$ & TSTEP $=1.2$ & PSTEP $=2.4$ \\
\hline $\mathrm{SB}$ & 1: & TIME & $=98.1$ & NTIME $=61$ & TSTEP $=2.2$ & PSTEP $=4.4$ \\
\hline SB & 1: & T IME & $=102.4$ & NTIME $=62$ & TSTEP $=4.3$ & PSTEP $=8.6$ \\
\hline SB & $1:$ & TIME & $=110.9$ & NTIME $=63$ & TSTEP $=8.5$ & PSTEP $=17.0$ \\
\hline SB & 1: & T IME & $=127.9$ & NT IME $=64$ & TSTEP $=17.0$ & PS TEP $=34.0$ \\
\hline SB & 1: & TIME & $=150.0$ & NTIME $=65$ & TSTEP $=22.1$ & PSTEP $=44.2$ \\
\hline SB & $1:$ & T IME & $=150.1$ & NTIME $=66$ & TSTEP $=0.1$ & PSTEP $=0.1$ \\
\hline$S B$ & 1: & TIME & $=150.2$ & NTIME $=67$ & TSTEP $=0.1$ & PSTEP $=0.2$ \\
\hline & UPEF & BLOCK & 1: $\quad$ T IME & STEP REJEC & PSTEP = & 0.1 \\
\hline SB & 1: & TIME & $=150.3$ & NTIME $=68$ & TSTEP $=0.1$ & PSTEP $=0.1$ \\
\hline SB & 1: & T IME & $=150.4$ & NTIME $=69$ & TSTEP $=0.1$ & PS TEP $=0.1$ \\
\hline$S B$ & 1: & TIME & $=150.5$ & NTIME $=70$ & TSTEP $=0.1$ & PSTEP $=0.1$ \\
\hline SB & $1:$ & T IME & $=150.6$ & NTIME $=71$ & TSTEP $=0.1$ & PSTEP $=0.1$ \\
\hline SB & $1:$ & TIME & $=150.7$ & NTIME $=72$ & TSTEP $=0.1$ & PSTEP $=0.1$ \\
\hline SB & $1:$ & T IME & $=150.8$ & NTIME $=73$ & TSTEP $=0.1$ & PSTEP $=0.1$ \\
\hline SB & $1:$ & TIME & $=150.9$ & NTIME $=74$ & TSTEP $=0.1$ & PSTEP $=0.1$ \\
\hline SB & 1: & T IME & $=151.0$ & NTIME $=75$ & TSTEP $=0.1$ & PS TEP $=0.1$ \\
\hline$S B$ & $1:$ & TIME & $=151.1$ & NTIME $=76$ & TSTEP $=0.1$ & PSTEP $=0.1$ \\
\hline SB & $1:$ & T IME & $=151.2$ & NTIME $=77$ & TSTEP $=0.1$ & PS TEP $=0.1$ \\
\hline SB & $1:$ & TIME & $=151.3$ & NTIME $=78$ & TSTEP $=0.1$ & PSTEP $=0.1$ \\
\hline $\mathrm{SB}$ & $1:$ & T IME & $=151.4$ & NTIME $=79$ & TSTEP $=0.1$ & PS TEP $=0.1$ \\
\hline SB & $1:$ & TIME & $=151.5$ & NTIME $=80$ & TSTEP $=0.1$ & PSTEP $=0.1$ \\
\hline SB & $1:$ & T IME & $=151.6$ & NTIME $=81$ & TSTEP $=0.1$ & PSTEP $=0.1$ \\
\hline $\mathrm{SB}$ & 1: & TIME & $=151.7$ & NTIME $=82$ & TSTEP $=0.1$ & PS TEP $=0.1$ \\
\hline SB & $1:$ & T IME & $=151.8$ & NT IME $=83$ & TSTEP $=0.1$ & PS TEP $=0.1$ \\
\hline SB & $1:$ & TIME & $=151.9$ & NTIME $=84$ & TSTEP $=0.1$ & PS TEP $=0.1$ \\
\hline SB & $1:$ & T IME & $=152.0$ & NTIME $=85$ & TSTEP $=0.1$ & PS TEP $=0.1$ \\
\hline SB & $1:$ & TIME & $=152.1$ & NTIME $=86$ & TSTEP $=0.1$ & PSTEP $=0.1$ \\
\hline SB & $1:$ & TIME & $=152.2$ & NT IME $=87$ & TSTEP $=0.1$ & PS TEP $=0.1$ \\
\hline SB & 1: & TIME & $=152.3$ & NTIME $=88$ & TSTEP $=0.1$ & PSTEP $=0.1$ \\
\hline SB & $1:$ & TIME & $=152.4$ & NTIME $=89$ & TSTEP $=0.1$ & PSTEP $=0.1$ \\
\hline SB & 1: & TIME & $=152.5$ & NTIME $=90$ & TSTEP $=0.1$ & PSTEP $=0.1$ \\
\hline SB & 1: & T IME & $=152.6$ & NTIME $=91$ & TSTEP $=0.1$ & PSTEP $=0.1$ \\
\hline SB & 1: & TIME & $=152.7$ & NTIME $=92$ & TSTEP $=0.1$ & PSTEP $=0.484044$ \\
\hline SB & 1: & T IME & $=153.1$ & NTIME $=93$ & TSTEP $=0.4$ & PS TEP $=0.341512$ \\
\hline$S B$ & $1:$ & TIME & $=153.4$ & NTIME $=94$ & TSTEP $=0.3$ & PSTEP $=0.6$ \\
\hline $\mathrm{SB}$ & . & TIME & $=154.0$ & NT IME $=95$ & TSTEP $=0.6$ & PSTEP $=0.868$ \\
\hline
\end{tabular}




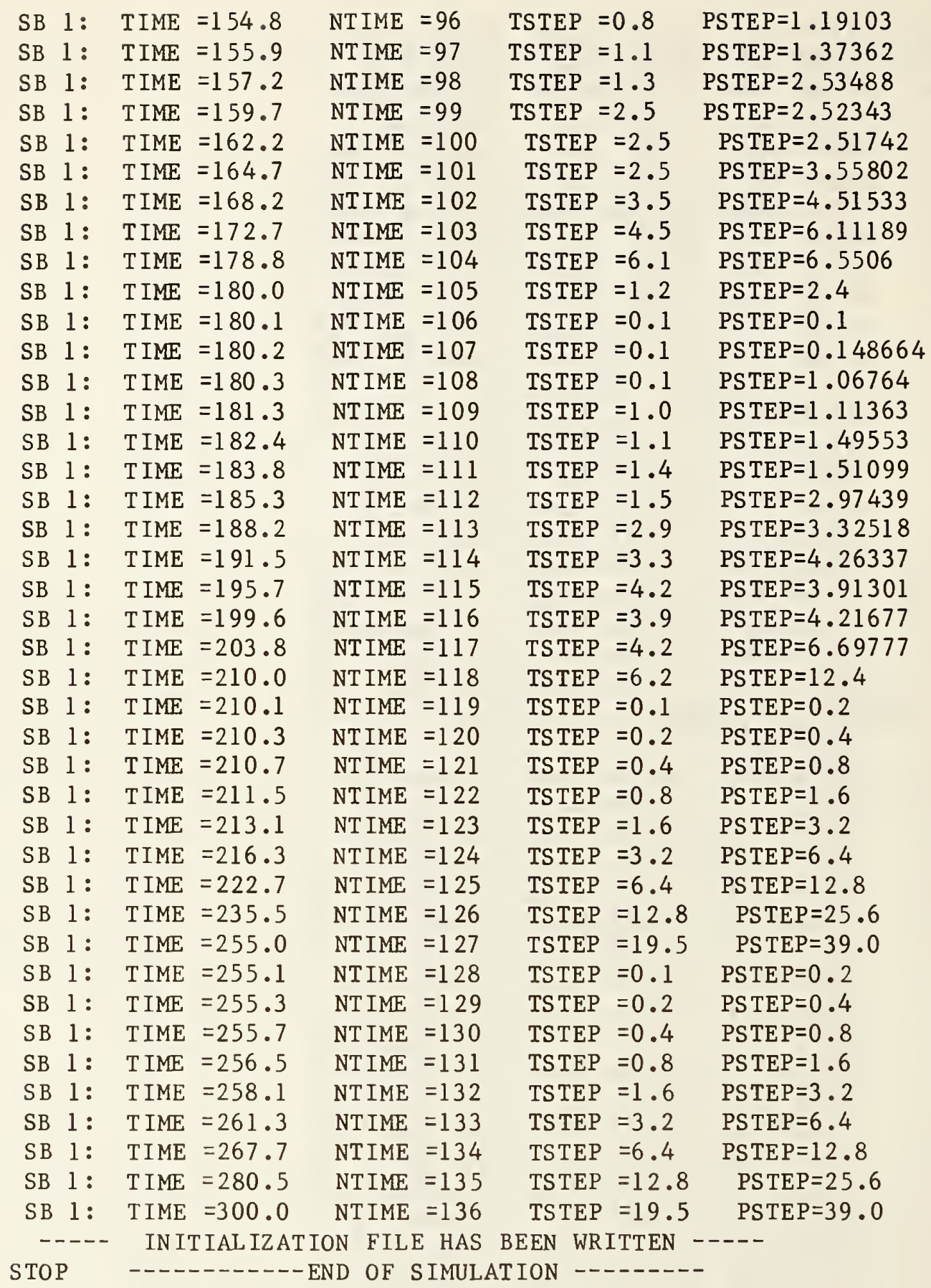




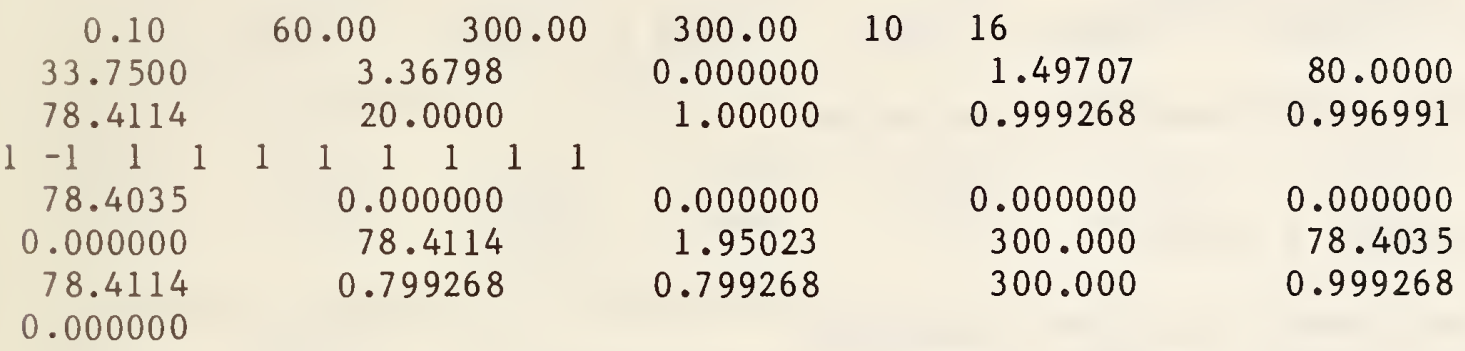

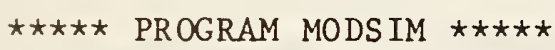

a MODular SIMulation program

EXAMPLE 1: INLET PIPE AND (NOMINALLY) LINEAR VALVE
1 SUPERBLOCKS
1 BLOCKS
2 UNITS

10 STATE VARIABLES:

3 PRES 1 FLOW 3 TEMP 3 CTRL

INITIAL STATE VECTOR:

PRES :
33.7500
3.00000
0.000000

FLOW :

2.00000

TEMP :

$80.0000 \quad 80.0000 \quad 20.0000$

CTRL :
1.00000
1.00000
1.00000

2 TIME DEPENDENT BOUNDARY VARIABLES:

TEMP 1 CTRL 1

ERROR TOLERANCES: RTOLX, ATOLX, XTOL, TTIME:
$5.00000 \mathrm{E}-03$
1. $00000 \mathrm{E}-05$
$1.00000 \mathrm{E}-03$
10.000

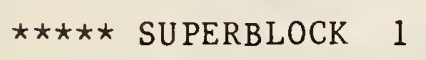


SUPERBLOCK SIMULTANEOUS EQUATION UNFREEZING OPTION, IFZOPT $=0$ SUPERBLOCK INPUT SCAN OPTION, INSOPT $=0$

7 REPORTED VARIABLES :

$\begin{array}{lllllll}\text { PRES } 2 & \text { FLOW } 1 & \text { TEMP } 1 & \text { TEMP } 2 & \text { CTRL } 1 & \text { CTRL } 2 & \text { CTRL } 3\end{array}$

O SIMULTANEOUS EQUATIONS; VARIABLES:

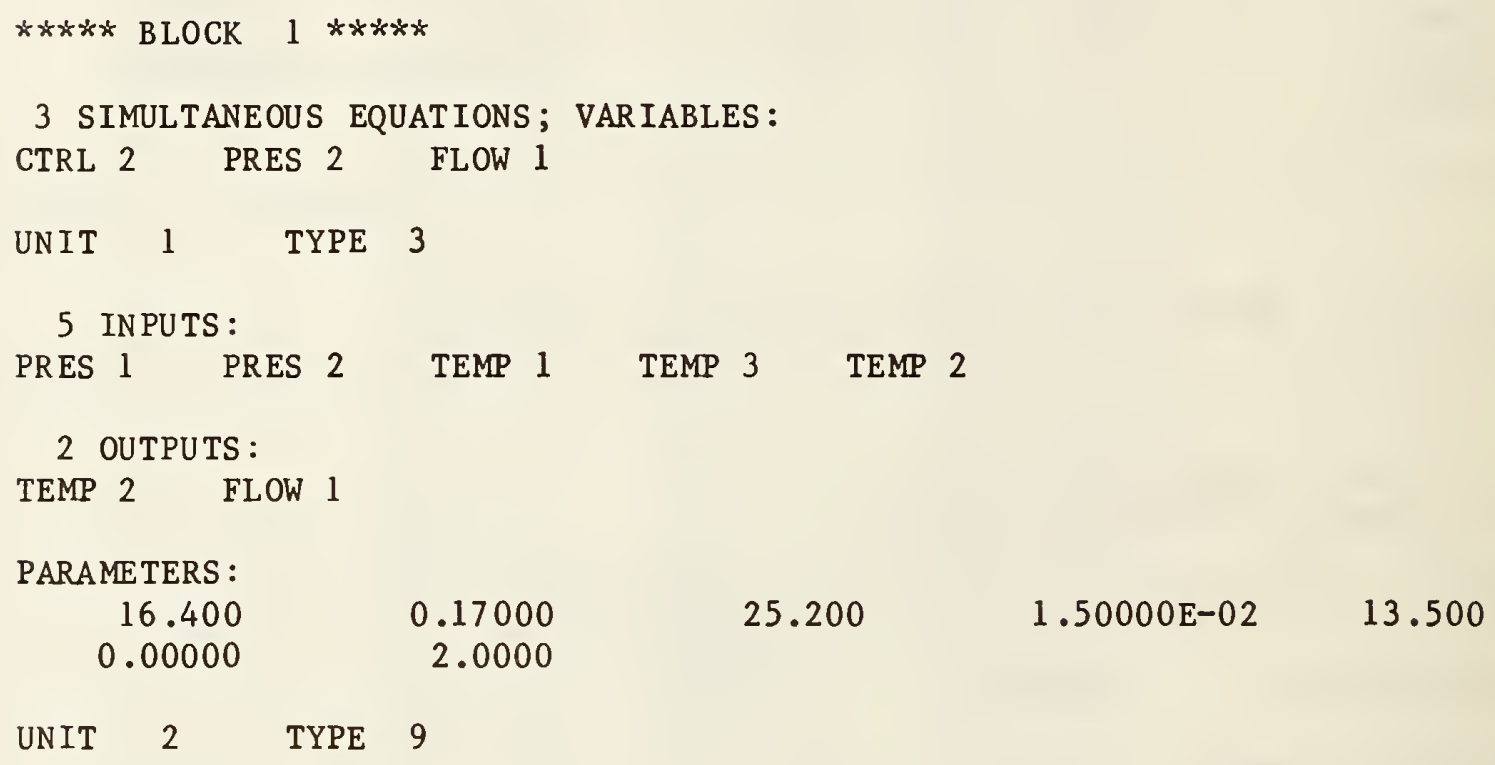

TMIN $=\quad 0.100$ TMAX $=\quad 60.000$ TS TOP $=300.000$

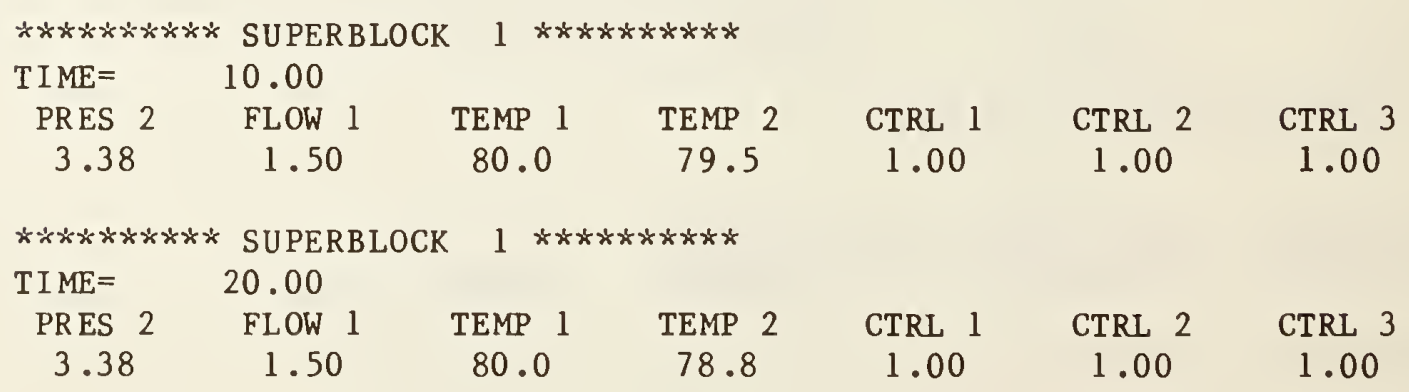




$\begin{array}{lcccccc}* \star * * * * * * * * & \text { SU PERBLOCK } & 1 & 1 * * * * * * * * * \\ \text { TIMEE }= & 30.00 & & & & & \\ \text { PRES 2 } & \text { FLOW 1 } & \text { TEMP 1 } & \text { TEMP 2 } & \text { CTRL 1 } & \text { CTRL 2 } & \text { CTRL 3 } \\ 3.38 & 1.50 & 80.0 & 78.4 & 1.00 & 1.00 & 1.00\end{array}$

$\begin{array}{cccclll}* * * * * * * * * * & \text { SUPERBLOCK } & 1 * * * * * * * * * * \\ \text { TIME }= & 40.00 & & & & \\ \text { PRES 2 } & \text { FLOW 1 } & \text { TEMP 1 } & \text { TEMP 2 } & \text { CTRL 1 } & \text { CTRL 2 } & \text { CTRL } 3 \\ 3.40 & 1.50 & 80.0 & 78.4 & 0.700 & 0.830 & 0.996\end{array}$

$\begin{array}{cccclll}* \star * * * * * * * * & \text { SUPERBLOCK } & 1 * * * * * * * * * * \\ \text { TIME }= & 50.00 & & & & & \\ \text { PRES 2 } & \text { FLOW 1 } & \text { TEMP 1 } & \text { TEMP 2 } & \text { CTRL 1 } & \text { CTRL 2 } & \text { CTRL } 3 \\ 6.57 & 1.42 & 80.0 & 78.4 & 0.400 & 0.546 & 0.683\end{array}$

$\begin{array}{lccclll}* * * * * * * * * * & \text { SUPERBLOCK } & 1 * * * * * * * * * * \\ \text { TIME }= & 60.00 & & & & & \\ \text { PRES 2 } & \text { FLOW 1 } & \text { TEMP 1 } & \text { TEMP 2 } & \text { CTRL 1 } & \text { CTRL 2 } & \text { CTRL } 3 \\ 17.9 & 1.08 & 80.0 & 78.4 & 0.100 & 0.249 & 0.312\end{array}$

$\begin{array}{ccccccc}* * * * * * * * * * & \text { SUPERBLOCK } & 1 * * * * * * * * * * * \\ \text { TIME }= & 70.00 & & & & & \\ \text { PRES 2 } & \text { FLOW 1 } & \text { TEMP 1 } & \text { TEMP 2 } & \text { CTRL 1 } & \text { CTRL 2 } & \text { CTRL } 3 \\ 27.9 & 0.660 & 80.0 & 78.3 & 0.100 & 0.120 & 0.151\end{array}$




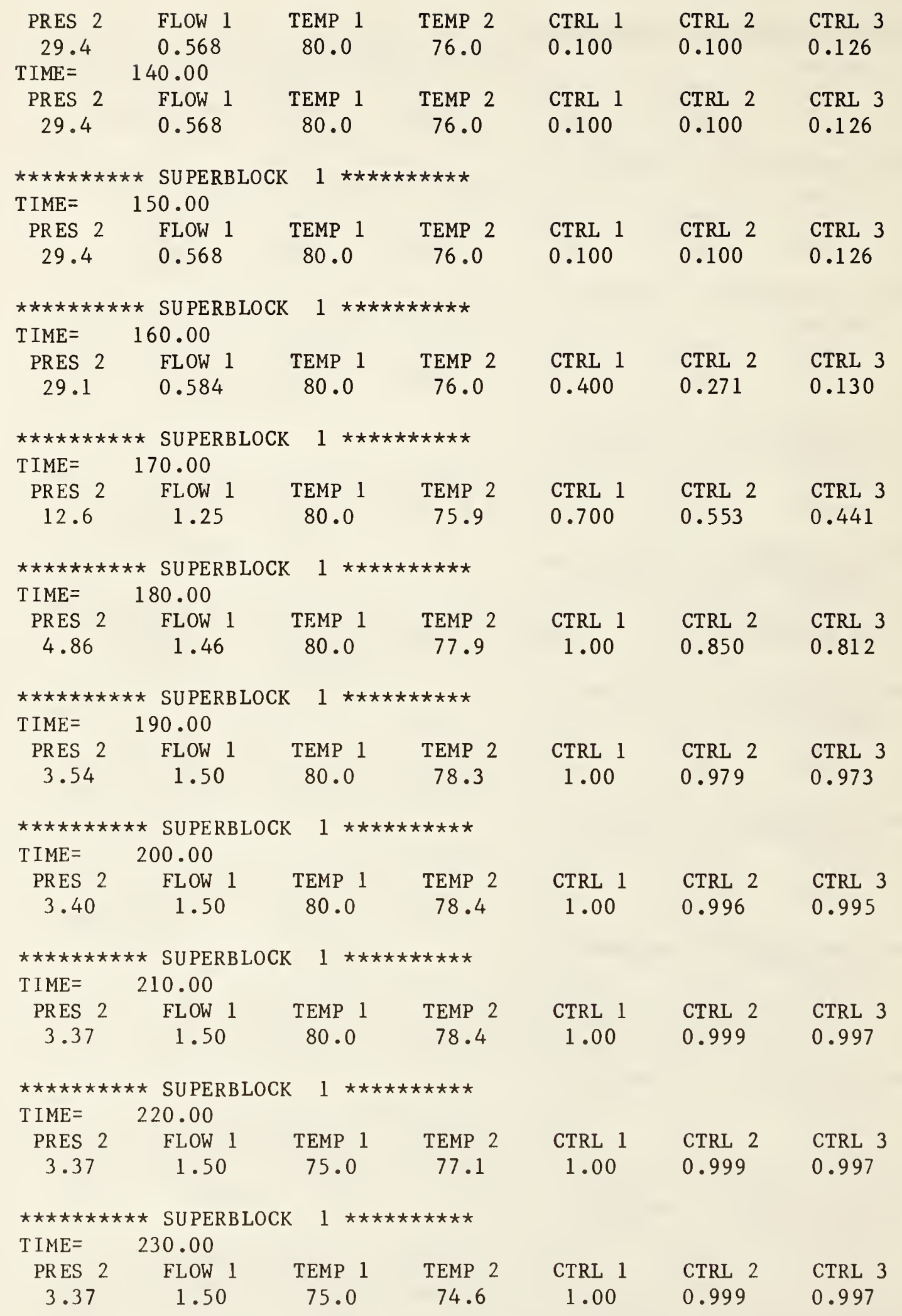

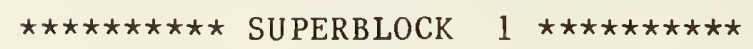




\begin{tabular}{|c|c|c|c|c|c|c|}
\hline $\mathrm{TIME}=$ & 240.00 & & & & & \\
\hline PRES 2 & FLOW 1 & TEMP 1 & TEMP 2 & CTRL 1 & CTRL 2 & CTRL 3 \\
\hline 3.37 & 1.50 & 75.0 & 73.6 & 1.00 & 0.999 & 0.997 \\
\hline TIME $=$ & 250.00 & & & & & \\
\hline PRES 2 & FLOW 1 & TEMP 1 & TEMP 2 & CTRL 1 & CTRL 2 & CTRL 3 \\
\hline 3.37 & 1.50 & 75.0 & 73.5 & 1.00 & 0.999 & 0.997 \\
\hline 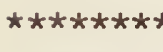 & ** SUPERB & $1 * *$ & $* * * * *$ & & & \\
\hline TIME $=$ & 260.00 & & & & & \\
\hline PRES 2 & FLOW 1 & TEMP 1 & TEMP 2 & CTRL 1 & CTRL 2 & CTRL 3 \\
\hline 3.37 & 1.50 & 80.0 & 73.5 & 1.00 & 0.999 & 0.997 \\
\hline$\star \star * * * * * * * x$ & ** SUPERB & $1 * *$ & $* * * * *$ & & & \\
\hline TIME $=$ & 270.00 & & & & & \\
\hline PRES 2 & FLOW 1 & TEMP 1 & TEMP 2 & CTRL 1 & CTRL 2 & CTRL 3 \\
\hline 3.37 & 1.50 & 80.0 & 76.4 & 1.00 & 0.999 & 0.997 \\
\hline $\mathrm{TIME}=$ & 280.00 & & & & & \\
\hline PRES 2 & FLOW 1 & TEMP 1 & TEMP 2 & CTRL 1 & CTRL 2 & CTRL 3 \\
\hline 3.37 & 1.50 & 80.0 & 78.3 & 1.00 & 0.999 & 0.997 \\
\hline 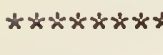 & ** SUPERB & 1 * & $\star \star * * * *$ & & & \\
\hline T I ME = & 290.00 & & & & & \\
\hline PRES 2 & FLOW 1 & TEMP 1 & TEMP 2 & CTRL 1 & CTRL 2 & CTRL 3 \\
\hline 3.37 & 1.50 & 80.0 & 78.4 & 1.00 & 0.999 & 0.997 \\
\hline $\mathrm{TIME}=$ & 300.00 & & & & & \\
\hline PRES 2 & FLOW 1 & TEMP 1 & TEMP 2 & CTRL 1 & CTRL 2 & CTRL 3 \\
\hline 3.37 & 1.50 & 80.0 & 78.4 & 1.00 & 0.999 & 0.997 \\
\hline
\end{tabular}




\begin{tabular}{|c|c|c|c|c|}
\hline SUPERBLOCK 1 & 0.10 & & & \\
\hline $\begin{array}{l}3.37500 \\
1.00000\end{array}$ & $\begin{array}{l}1.50000 \\
1.00000\end{array}$ & 79.9999 & 79.9605 & 0.999999 \\
\hline SUPERBLOCK 1 & 0.30 & & & \\
\hline 3.37500 & 1.50000 & 80.0000 & 79.9605 & 1.00000 \\
\hline 1.00000 & 1.00000 & & & \\
\hline SUPERBLOCK 1 & 0.70 & & & \\
\hline 3.37500 & 1.50000 & 80.0000 & 79.9365 & 1.00000 \\
\hline 1.00000 & 1.00000 & & & \\
\hline SUPERBLOCK 1 & 1.50 & & & \\
\hline 3.37500 & 1.50000 & 80.0000 & 79.9523 & 1.00000 \\
\hline 1.00000 & 1.00000 & & & \\
\hline SUPERBLOCK 1 & 3.10 & & & \\
\hline 3.37500 & 1.50000 & 80.0001 & 79.9767 & 1.00000 \\
\hline 1.00000 & 1.00000 & & & \\
\hline SUPERBLOCK 1 & 6.30 & & & \\
\hline 3.37500 & 1.50000 & 79.9999 & 79.9691 & 0.999999 \\
\hline 1.00000 & 1.00000 & & & \\
\hline SUPERBLOCK 1 & 12.70 & & & \\
\hline 3.37500 & 1.50000 & 80.0000 & 79.2213 & 1.00000 \\
\hline 1.00000 & 1.00000 & & & \\
\hline SUPERBLOCK 1 & 25.50 & & & \\
\hline 3.37500 & 1.50000 & 80.0000 & 78.4139 & 1.00000 \\
\hline 1.00000 & 1.00000 & & & \\
\hline SUPERBLOCK 1 & 30.00 & & & \\
\hline 3.37500 & 1.50000 & 80.0000 & 78.4139 & 1.00000 \\
\hline 1.00000 & 1.00000 & & & \\
\hline SUPERBLOCK 1 & 30.10 & & & \\
\hline 3.37500 & 1.50000 & 80.0000 & 78.4139 & 0.997000 \\
\hline 1.00000 & 1.00000 & & & \\
\hline SUPERBLOCK 1 & 30.30 & & & \\
\hline 3.37500 & 1.50000 & 80.0000 & 78.4139 & 0.991000 \\
\hline 0.999640 & 1.00000 & & & \\
\hline SUPERBLOCK 1 & 30.40 & & & \\
\hline 3.37500 & 1.50000 & 80.0000 & 78.4139 & 0.988000 \\
\hline 0.999615 & 1.00000 & & & \\
\hline SUPERBLOCK 1 & 30.60 & & & \\
\hline 3.37500 & 1.50000 & 80.0000 & 78.4139 & 0.982000 \\
\hline 0.999508 & 1.00000 & & & \\
\hline SUPERBLOCK 1 & 30.80 & & & \\
\hline 3.37500 & 1.50000 & 80.0000 & 78.4139 & 0.976000 \\
\hline 0.998892 & 1.00000 & & & \\
\hline SUPERBLOCK 1 & 30.90 & & & \\
\hline 3.37500 & 1.50000 & 80.0000 & 78.4139 & 0.973000 \\
\hline 0.998157 & 1.00000 & & & \\
\hline SUPERBLOCK 1 & 31.00 & & & \\
\hline 3.37500 & 1.50000 & 80.0000 & 78.4139 & 0.970000 \\
\hline 0.997313 & 1.00000 & & & \\
\hline
\end{tabular}


SUPERBLOCK 1

3.37500

0.996534

SUPERBLOCK 1

3.37500

0.995844

SUPERBLOCK 1

3.37500

0.995174

SUPERBLOCK 1

3.37500

0.994476

SUPERBLOCK 1

3.37500

0.992947

SU PERBLOCK 1

3.37500

0.991234

SU PERBLOCK 1

3.37500

0.981570

SUPERBLOCK 1

3.37500

0.964446

SU PERBLOCK 1

3.37500

0.939523

SUPERBLOCK 1

3.37500

0.906027

SUPERBLOCK 1

3.37500

0.835452

SU PERBLOCK 1

3.82271

0.745964

SUPERBLOCK 1

5.22577

0.622457

SUPERBLOCK 1

7.14338

0.513745

SUPERBLOCK 1

10.0897

0.407117

SU PERBLOCK 1

16.9213

0.264244

SUPERBLOCK 1

17.8919

0.249316

SUPERBLOCK 1
31.10

1.50000

80.0000

78.4139

0.967000

1.00000

31.20

1.50000

80.0000

78.4139

0.964001

1.00000

31.30

1.50000

80.0000

78.4139

0.961000

1.00000

31.40

1.50000

80.0000

78.4139

0.958000

1.00000

31.60

1.50000

1.00000

31.80

1.50000

1.00000

32.70

1.50000

1.00000

33.90

1.50000

1.00000

35.30

1.50000

1.00000

36.90

1.50000

1.00000

39.80

1.50000

1.00000

43.10

1.48890

0.932454

47.40

1.45358

0.778071

51.10

1.40388

0.642182

54.70

1.32387

0.508896

59.50

1.11650

80.0000

78.4139

0.952000

80.0000

78.4139

0.946000

80.0000

78.4139

0.919001

80.0000

78.4139

0.883000

80.0000

78.4139

0.841000

80.0000

78.4139

0.793000

80.0000

78.4139

0.706000

80.0000

78.4117

0.607000

80.0000

78.4115

0.478001

80.0000

78.4092

0.367001

80.0000

78.3994

0.259001

80.0000

78.3733

0.115000

0.330305

60.00

1.08384

0.311646

80.0000

78.3791

$9.999996 E-02$

60.10 


\begin{tabular}{|c|c|c|c|c|}
\hline 18.0881 & 1.07710 & 80.0000 & 78.3786 & $9.999996 E-02$ \\
\hline 0.246389 & 0.307986 & & & \\
\hline SUPERBLOCK 1 & 60.20 & & & \\
\hline 18.2827 & 1.07039 & 80.0000 & 78.3779 & $9.999996 \mathrm{E}-02$ \\
\hline 0.243518 & 0.304398 & & & \\
\hline SUPERBLOCK 1 & 60.30 & & & \\
\hline 18.4753 & 1.06370 & 80.0000 & 78.3770 & $9.999996 \mathrm{E}-02$ \\
\hline 0.240704 & 0.300880 & & & \\
\hline SUPERBLOCK 1 & 60.80 & & & \\
\hline 19.4129 & 1.03055 & 80.0000 & 78.3716 & $9.999996 E-02$ \\
\hline 0.227362 & 0.284202 & & & \\
\hline SUPERBLOCK 1 & 61.20 & & & \\
\hline 20.1259 & 1.00460 & 80.0000 & 78.3659 & $9.999996 \mathrm{E}-02$ \\
\hline 0.217566 & 0.271958 & & & \\
\hline SUPERBLOCK 1 & 62.00 & & & \\
\hline 21.4438 & 0.954763 & 80.0000 & 78.3550 & $9.999996 \mathrm{E}-02$ \\
\hline 0.200118 & 0.250148 & & & \\
\hline SUPERBLOCK 1 & 62.70 & & & \\
\hline 22.4740 & 0.913977 & 80.0000 & 78.3462 & $9.999996 \mathrm{E}-02$ \\
\hline 0.186960 & 0.233700 & & & \\
\hline SUPERBLOCK 1 & 64.10 & & & \\
\hline 24.1882 & 0.841588 & 80.0000 & 78.3348 & $9.999996 \mathrm{E}-02$ \\
\hline 0.165659 & 0.207074 & & & \\
\hline SUPERBLOCK 1 & 65.90 & & & \\
\hline 25.8064 & 0.767069 & 80.0000 & 78.3229 & $9.999996 \mathrm{E}-02$ \\
\hline 0.145884 & 0.182355 & & & \\
\hline SUPERBLOCK 1 & 66.00 & & & \\
\hline 25.8799 & 0.763523 & 80.0000 & 78.3225 & $9.999996 \mathrm{E}-02$ \\
\hline 0.144984 & 0.181230 & & & \\
\hline SUPERBLOCK 1 & 66.10 & & & \\
\hline 25.9521 & 0.760015 & 80.0000 & 78.3226 & $9.999996 \mathrm{E}-02$ \\
\hline 0.144102 & 0.180127 & & & \\
\hline SUPERBLOCK 1 & 66.20 & & & \\
\hline 26.0228 & 0.756562 & 80.0000 & 78.3227 & $9.999996 \mathrm{E}-02$ \\
\hline 0.143237 & 0.179046 & & & \\
\hline SUPERBLOCK 1 & 66.90 & & & \\
\hline 26.4826 & 0.733730 & 80.0000 & 78.3204 & $9.999996 \mathrm{E}-02$ \\
\hline 0.137605 & 0.172006 & & & \\
\hline SUPERBLOCK 1 & 67.50 & & & \\
\hline 26.8285 & 0.716043 & 80.0000 & 78.3154 & $9.999996 \mathrm{E}-02$ \\
\hline 0.133342 & 0.166678 & & & \\
\hline SUPERBLOCK 1 & 68.40 & & & \\
\hline 27.2745 & 0.692609 & 80.0000 & 78.3057 & $9.999996 \mathrm{E}-02$ \\
\hline 0.127816 & 0.159769 & & & \\
\hline SUPERBLOCK 1 & 69.40 & & & \\
\hline 27.6815 & 0.670485 & 80.0000 & 78.2925 & $9.999996 \mathrm{E}-02$ \\
\hline 0.122721 & 0.153401 & & & \\
\hline SUPERBLOCK 1 & 71.30 & & & \\
\hline 28.2498 & 0.638295 & 80.0000 & 78.2645 & $9.999996 \mathrm{E}-02$ \\
\hline 0.115503 & 0.144378 & & & \\
\hline SUPERBLOCK 1 & 73.30 & & & \\
\hline 28.6431 & 0.615103 & 80.0000 & 78.2308 & $9.999996 \mathrm{E}-02$ \\
\hline
\end{tabular}




\begin{tabular}{|c|c|c|c|c|}
\hline 0.110429 & 0.138036 & & & \\
\hline SUPERBLOCK 1 & 76.40 & & & \\
\hline 28.9975 & 0.593323 & 80.0000 & 78.1716 & $9.999996 \mathrm{E}-02$ \\
\hline 0.105762 & 0.132203 & & & \\
\hline SUPERBLOCK 1 & 78.80 & & & \\
\hline 29.1536 & 0.583511 & 80.0000 & 78.1125 & $9.999996 \mathrm{E}-02$ \\
\hline 0.103686 & 0.129607 & & & \\
\hline SU PERBLOCK 1 & 81.00 & & & \\
\hline 29.2462 & 0.577597 & 80.0000 & 78.0479 & $9.999996 \mathrm{E}-02$ \\
\hline 0.102443 & 0.128053 & & & \\
\hline SUPERBLOCK 1 & 84.40 & & & \\
\hline 29.3354 & 0.571845 & 80.0000 & 77.9225 & $9.999996 \mathrm{E}-02$ \\
\hline 0.101239 & 0.126549 & & & \\
\hline SUPERBLOCK 1 & 88.30 & & & \\
\hline $\begin{array}{r}29.3895 \\
0.100506\end{array}$ & 0.568330 & 80.0000 & 77.7289 & $9.999996 \mathrm{E}-02$ \\
\hline $\begin{array}{c}0.100506 \\
\text { SUPERBLOCK } 1\end{array}$ & $\begin{array}{l}0.125633 \\
92.80\end{array}$ & & & \\
\hline $\begin{array}{c}\text { SUPERBLOCK } 1 \\
29.3895\end{array}$ & $\begin{array}{l}92.80 \\
0.568332\end{array}$ & 80.0000 & 77.4340 & $9.999996 \mathrm{E}-02$ \\
\hline 0.100805 & 0.125633 & & & \\
\hline SUPERBLOCK 1 & 94.10 & & & \\
\hline 29.3897 & 0.568332 & 80.0000 & 77.3390 & $9.999996 \mathrm{E}-02$ \\
\hline 0.100639 & 0.125633 & & & \\
\hline SUPERBLOCK 1 & 94.70 & & & \\
\hline 29.3897 & 0.568332 & 80.0000 & 77.2934 & $9.999996 \mathrm{E}-02$ \\
\hline $\begin{array}{c}0.100570 \\
0\end{array}$ & & & & \\
\hline $\begin{array}{c}\text { SUPERBLOCK } 1 \\
29.3897\end{array}$ & $\begin{array}{l}95.90 \\
0.568332\end{array}$ & 80.0000 & 77.2003 & $9.999996 \mathrm{E}-02$ \\
\hline 0.100460 & 0.125633 & & & \\
\hline SUPERBLOCK 1 & 98.10 & & & \\
\hline $\begin{array}{r}29.3897 \\
0.100460\end{array}$ & $\begin{array}{l}0.568332 \\
0.125633\end{array}$ & 80.0000 & 77.0291 & $9.999996 \mathrm{E}-02$ \\
\hline SUPERBLOCK 1 & 102.40 & & & \\
\hline 29.3897 & 0.568332 & 80.0000 & 76.7190 & $9.999996 \mathrm{E}-02$ \\
\hline 0.100460 & 0.125633 & & & \\
\hline SUPERBLOCK 1 & 110.90 & & & \\
\hline $\begin{array}{r}29.3897 \\
0.100460\end{array}$ & $\begin{array}{l}0.568332 \\
0.125633\end{array}$ & 80.0000 & 76.2880 & $9.999996 \mathrm{E}-02$ \\
\hline SU PERBLOCK 1 & 127.90 & & & \\
\hline 29.3897 & 0.568332 & 80.0000 & 75.9746 & $9.999996 \mathrm{E}-02$ \\
\hline 0.100460 & 0.125633 & & & \\
\hline SUPERBLOCK 1 & 150.00 & & & \\
\hline 29.3897 & 0.568332 & 80.0000 & 75.9746 & $9.999996 \mathrm{E}-02$ \\
\hline 0.100460 & 0.125633 & & & \\
\hline SUPERBLOCK 1 & 150.10 & & & \\
\hline 29.3897 & 0.568332 & 80.0000 & 75.9746 & 0.103000 \\
\hline 0.100416 & 0.125633 & & & \\
\hline SUPERBLOCK 1 & 150.20 & & & \\
\hline 29.3897 & 0.568332 & 80.0000 & 75.9746 & 0.105999 \\
\hline 0.100428 & 0.125633 & & & \\
\hline SUPERBLOCK 1 & 150.30 & 80.0000 & 75.9746 & 0.108998 \\
\hline $\begin{array}{r}29.3897 \\
0.100596\end{array}$ & $\begin{array}{l}0.568332 \\
0.125633\end{array}$ & 00.0000 & ש ק & \\
\hline
\end{tabular}




\begin{tabular}{|c|c|c|c|c|}
\hline SUPERBLOCK 1 & 150.40 & & & \\
\hline $\begin{array}{r}29.3897 \\
0.100820\end{array}$ & $\begin{array}{l}0.568332 \\
0.125633\end{array}$ & 80.0000 & 75.9746 & 0.111998 \\
\hline $\begin{array}{l}0.100820 \\
\text { SUPERB LOCK } 1\end{array}$ & $\begin{array}{c}0.125633 \\
150.50\end{array}$ & & & \\
\hline 29.3897 & 0.568332 & 80.0000 & 75.9746 & 0.114998 \\
\hline 0.101098 & 0.125633 & & & \\
\hline SUPERBLOCK 1 & 150.60 & & & \\
\hline 29.3897 & 0.568332 & 80.0000 & 75.9746 & 0.117998 \\
\hline 0.101429 & 0.125633 & & & \\
\hline SUPERBLOCK 1 & 150.70 & & & \\
\hline 29.3897 & 0.568332 & 80.0000 & 75.9746 & 0.120999 \\
\hline 0.101813 & 0.125633 & & & \\
\hline SUPERBLOCK 1 & 150.80 & & & \\
\hline 29.3897 & 0.568332 & 80.0000 & 75.9746 & 0.123998 \\
\hline 0.102248 & 0.125633 & & & \\
\hline SUPERBLOCK 1 & 150.90 & & & \\
\hline 29.3897 & 0.568332 & 80.0000 & 75.9746 & 0.126998 \\
\hline 0.102733 & 0.125633 & & & \\
\hline SUPERBLOCK 1 & 151.00 & & & \\
\hline 29.3897 & 0.568332 & 80.0000 & 75.9746 & 0.129998 \\
\hline 0.103268 & 0.125633 & & & \\
\hline SUPERBLOCK 1 & 151.10 & & & \\
\hline 29.3897 & 0.568332 & 80.0000 & 75.9746 & 0.132998 \\
\hline 0.103851 & 0.125633 & & & \\
\hline SUPERBLOCK 1 & 151.20 & & & \\
\hline 29.3897 & 0.568332 & 80.0000 & 75.9746 & 0.135998 \\
\hline 0.104481 & 0.125633 & & & \\
\hline SUPERBLOCK 1 & 151.30 & & & \\
\hline 29.3897 & 0.568332 & 80.0000 & 75.9746 & 0.138998 \\
\hline 0.105158 & 0.125633 & & & \\
\hline SUPERBLOCK 1 & 151.40 & & & \\
\hline 29.3897 & 0.568332 & 80.0000 & 75.9746 & 0.141998 \\
\hline 0.105880 & 0.125633 & & & \\
\hline SUPERBLOCK 1 & 151.50 & & & \\
\hline 29.3897 & 0.568332 & 80.0000 & 75.9746 & 0.144998 \\
\hline 0.106647 & 0.125633 & & & \\
\hline SUPERBLOCK 1 & 151.60 & & & \\
\hline 29.3897 & 0.568332 & 80.0000 & 75.9746 & 0.147998 \\
\hline 0.107458 & 0.125633 & & & \\
\hline SUPERBLOCK 1 & 151.70 & & & \\
\hline 29.3897 & 0.568332 & 80.0000 & 75.9746 & 0.150999 \\
\hline 0.108312 & 0.125633 & & & \\
\hline SUPERBLOCK 1 & 151.80 & & & \\
\hline 29.3897 & 0.568332 & 80.0000 & 75.9746 & 0.153998 \\
\hline 0.109208 & 0.125633 & & & \\
\hline SUPERBLOCK 1 & 151.90 & & & \\
\hline 29.3897 & 0.568332 & 80.0000 & 75.9746 & 0.156998 \\
\hline 0.110145 & 0.125633 & & & \\
\hline SUPERBLOCK 1 & 152.00 & & & \\
\hline 29.3897 & 0.568332 & 80.0000 & 75.9746 & 0.159998 \\
\hline 0.111122 & 0.125633 & & & \\
\hline SUPERBLOCK 1 & 152.10 & & & \\
\hline
\end{tabular}




\subsection{7 \\ 0.112139}

SUPERBLOCK 1

29.3897

0.113195

SUPERBLOCK 1

29.3897

0.114290

SUPERBLOCK 1

29.3897

0.115421

SUPERBLOCK 1

29.3897

0.116589

SUPERBLOCK 1 29.3897

0.117794

SUPERBLOCK 1 29.3897

0.119033

SUPERBLOCK 1 29.3897

0.124290

SUPERBLOCK 1 29.3897

0.128557

SUPERBLOCK 1 29.3897

0.137883

SUPERBLOCK 1 29.3897

0.151793

SU PERBLOCK 1 29.3897

0.173341

SUPERBLOCK 1 29.3897

0.201738

SUPERBLOCK 1 29.3897

0.262821

SUPERBLOCK 1

27.1418

0.329470

SUPERBLOCK 1

21.4995

0.399396

SUPERBLOCK 1

14.7971

0.500222

SUPERBLOCK 1

$$
0.568332
$$

0.125633

152.20

0.568332

0.125633

152.30

$$
0.568332
$$

0.125633

152.40

0.568332

0.125633

152.50

0.568332

0.125633

152.60

0.568332

0.125633

152.70

0.568332

0.125633

153.10

0.568332

0.125633

153.40

0.568332

0.125633

154.00

0.568332

0.125633

154.80

0.568332

0.125633

155.90

0.568332

0.125633

157.20

0.568332

0.125633

159.70

0.568332

0.125633

162.20

0.699634

0.161838

164.70

0.952597

0.249246

168.20

1.18486

0.375278

172.70

1.34683
80.0000

75.9746

0.162998

80.0000

75.9746

0.165999

80.0000

75.9746

0.168998

80.0000

75.9746

0.171998

80.0000

75.9746

0.174998

80.0000

75.9746

0.177998

80.0000

75.9746

0.180999

80.0000

75.9746

0.192998

80.0000

75.9746

0.201998

80.0000

75.9746

0.219998

80.0000

75.9746

0.243998

80.0000

75.9746

0.276998

80.0000

75.9746

0.315999

80.0000

75.9746

0.390998

80.0000

75.9010

0.465998

80.0000

75.9043

0.540998

80.0000

75.9078

0.645998

80.0000

75.8956

0.780998 


\begin{tabular}{|c|c|c|c|c|}
\hline 0.632450 & 0.540562 & & & \\
\hline SUPERBLOCK 1 & 178.80 & & & \\
\hline 5.34785 & 1.45047 & 80.0000 & 77.2652 & 0.963998 \\
\hline 0.813958 & 0.767448 & & & \\
\hline SUPERBLOCK 1 & 180.00 & & & \\
\hline 4.85705 & 1.46295 & 80.0000 & 77.9301 & 1.00000 \\
\hline $\begin{array}{c}0.849922 \\
\text { SIIPFRBI }\end{array}$ & 0.812403 & & & \\
\hline $\begin{array}{c}\text { SUPERBLOCK } 1 \\
4.81975\end{array}$ & $\begin{array}{l}180.10 \\
1.46389\end{array}$ & & & \\
\hline & 0.816081 & 00.0000 & 77.9334 & 1.00000 \\
\hline SUPERBLOCK 1 & 180.20 & & & \\
\hline 4.77761 & 1.46389 & 80.0000 & 77.9369 & 1.00000 \\
\hline 0.855750 & 0.819687 & & & \\
\hline SUPERBLOCK 1 & 180.30 & & & \\
\hline 4.73681 & 1.46389 & 80.0000 & 77.9404 & 1.00000 \\
\hline 0.858578 & 0.823223 & & & \\
\hline SUPERBLOCK 1 & 181.30 & & & \\
\hline 4.44679 & 1.47330 & 80.0000 & 77.9763 & 1.00000 \\
\hline 0.884180 & 0.855225 & & & \\
\hline SUPERBLOCK 1 & 182.40 & & & \\
\hline 4.19778 & 1.47954 & 80.0000 & 78.0163 & 1.00000 \\
\hline 0.907251 & 0.884064 & & & \\
\hline SUPERBLOCK 1 & 183.80 & & & \\
\hline 3.96843 & 1.48 .528 & 80.0000 & 78.0663 & 1.00000 \\
\hline 0.930302 & 0.912878 & & & \\
\hline SUPERBLOCK 1 & 185.30 & & & \\
\hline 3.79680 & 1.48955 & 80.0000 & 78.1202 & 1.00000 \\
\hline 0.948838 & 0.936047 & & & \\
\hline SUPERBLOCK 1 & 188.20 & & & \\
\hline 3.60080 & 1.49441 & 80.0000 & 78.2244 & 1.00000 \\
\hline 0.971536 & 0.964421 & & & \\
\hline SU PERBLOCK 1 & 191.50 & & & \\
\hline 3.49352 & 1.49707 & 80.0000 & 78.3118 & 1.00000 \\
\hline 0.984729 & 0.980912 & & & \\
\hline SUPERBLOCK 1 & 195.70 & & & \\
\hline 3.42751 & 1.49707 & 80.0000 & 78.3677 & 1.00000 \\
\hline 0.992274 & 0.990343 & & & \\
\hline SUPERBLOCK 1 & 199.60 & & & \\
\hline 3.39912 & 1.49707 & 80.0000 & 78.3911 & 1.00000 \\
\hline 0.995586 & 0.994483 & & & \\
\hline SUPERBLOCK 1 & 203.80 & & & \\
\hline 3.38209 & 1.49707 & 80.0000 & 78.4025 & 1.00000 \\
\hline 0.997593 & 0.996991 & & & \\
\hline SUPERBLOCK 1 & 210.00 & & & \\
\hline 3.36798 & 1.49707 & 80.0000 & 78.4092 & 1.00000 \\
\hline 0.999268 & 0.996991 & & & \\
\hline SUPERBLOCK 1 & 210.10 & & & \\
\hline 3.36798 & 1.49707 & 75.0000 & 78.4104 & 1.00000 \\
\hline 0.999268 & 0.996991 & & & \\
\hline SUPERBLOCK 1 & 210.30 & & & \\
\hline 3.36798 & 1.49707 & 75.0000 & 78.3619 & 1.00000 \\
\hline 0.999268 & 0.996991 & & & \\
\hline
\end{tabular}




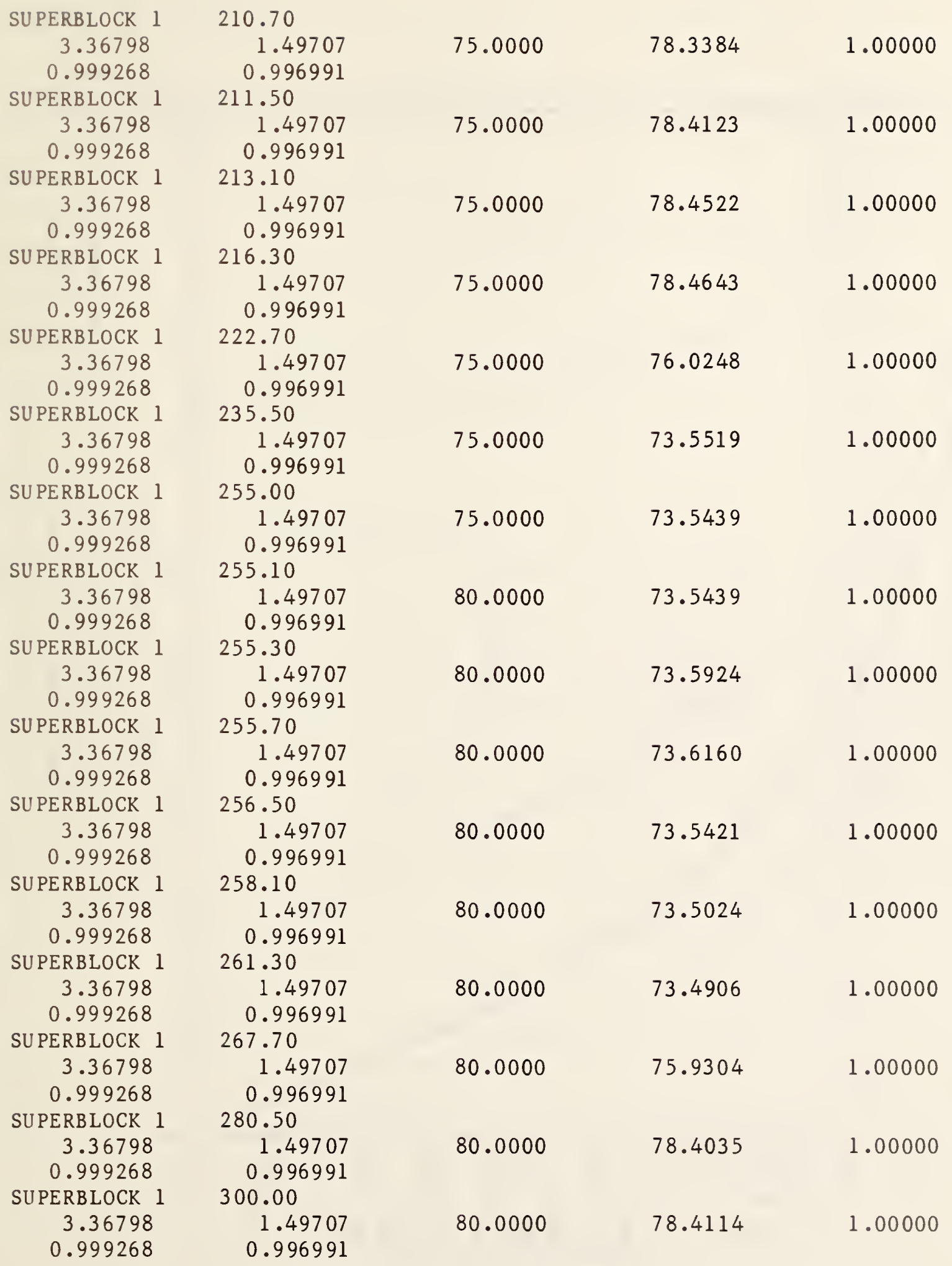




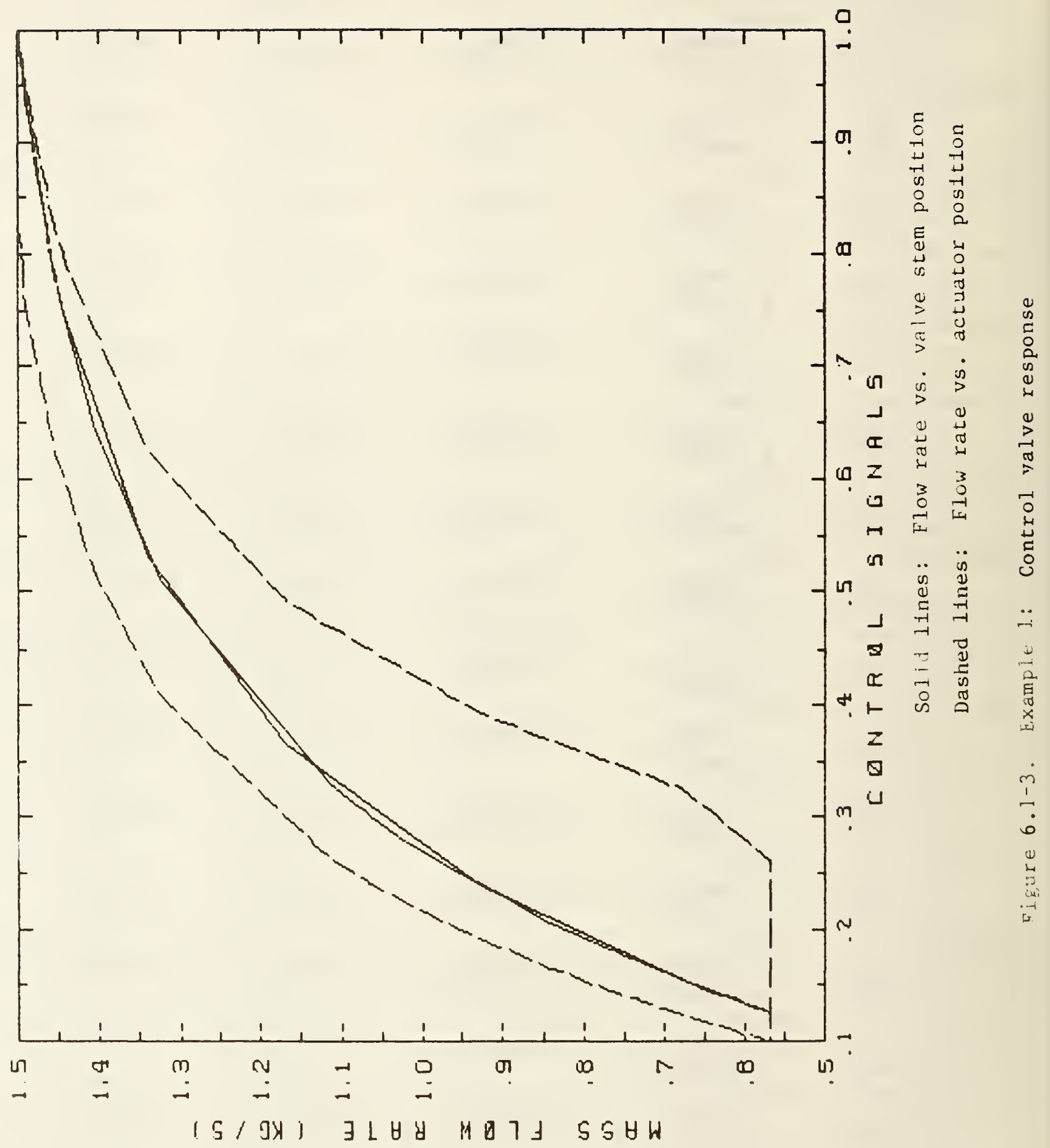




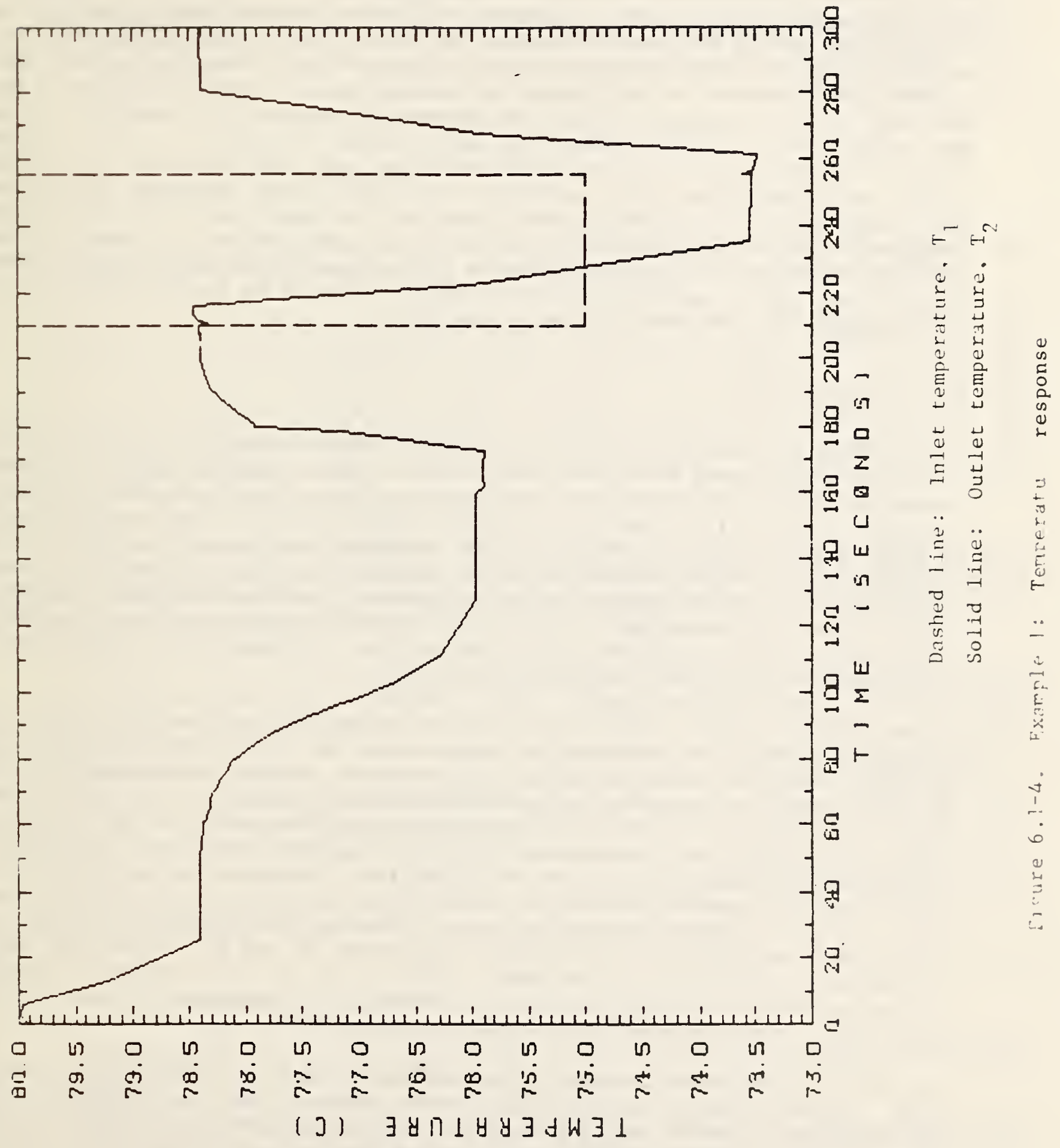




\subsection{Example 2: Heating Coil Control Loop, Variable Inlet Water Temperature}

This example represents a system in which the hot water flow rate through a heat exchanger is kept nearly constant, and control is achieved by varying the temperature of the hot water entering the coil. The piping arrangement for such a system is shown schematically in Figure 6.2-1. When the control port of the control valve is fully open, hot supply water from the boiler is fed directly to the heating coil and the system operates at full capacity. When the control port is fully closed, hot supply water circulates in the left-hand loop and unheated water circulates through the coil and the shunt port of the valve. At intermediate valve positions, hot supply water entering the control port mixes with recirculating water entering the shunt port to determine the temperature of the water entering the coil. In such systems the control valve is placed close to the coil to minimize the transport time around the recirculation loop, and a non-linear valve is often selected to help compensate for the inherent non-linearity of the system [3].

\subsubsection{Configuration}

To simplify the example, the portion of the flow circuit to the left of the dotted line in Figure 6.2-1 has been omitted. The resulting system is shown schematically in Figure 6.2-2, with unit numbers, type numbers, and state variables identified. The simulation configuration is also summarized in Listing 6.2-1. The net effect of the omitted portion of the circuit is approximated by the constant difference between pressures $\mathrm{Pl}$ and $\mathrm{P} 8$. These two pressures are not outputs of any components, and therefore are time independent boundary conditions. The initial values assigned to these variables will remain fixed throughout the simulation. A temperature sensor and a controller have also been added to the system.

In designing a simulation, particularly one which involves multiple flow streams, care must be exercised to ensure that the set of equations defining pressures and flow rates throughout the system is neither underdetermined nor overdetermined. In general, at least one pressure should be a boundary condition to provide a base from which pressure differences can be computed. Often, as in this case, two pressures will be boundary conditions, providing a fixed pressure difference to drive fluid flow. In addition, each flow rate which is not a boundary condition should be an output of exactly one component. In this example two UNITS of the TYPE 3 inlet pipe model (UNITS 1 and 3) have been selected to calculate the flow rates $W 1$ and $W 2$. Flow rate W3 is calculated in the TYPE 13 valve model, UNIT 2, as the sum of flows W1 and W2. On the air side of the Unit 6 , Type 11 heating coil, pressure P10 and flow W4 are boundary conditions which determine pressure P9.

The UNIT 3, TYPE 3 pipe is used only to calculate flow rate W2 in this simulation. Thermal losses, thermal capacitance effects, and the transport delay have been turned off by setting the first four parameters of the UNIT to zero. In addition, the pipe's out let temperature has been removed from the set of simultaneous equations by assigning it an index number of zero. The pipe's inlet temperature, $\mathrm{T} 4$, is also the inlet temperature for the next component downstream, which is the UNIT 2, TYPE 13 valve. HVACSIM allows any number of 
inputs and outputs to be "zeroed out" of a simulation.

The procedure for using HVACGEN to create a simulation has been presented in detail in the previous example, and will not be repeated here. Listing 6.2-1 was generated by HVACGEN in response to the command VIEW SIM EXAMPLE2 ALL. The information in this listing may be used to recreate the simulation. Alternatively, the simulation work file given in Listing 6.2-2 may be used directly as input to either HVACGEN or SLIMCON.

\subsubsection{Execution}

This example represents a well-tuned control system. Tuning of the system was accomplished by trial and error, as outlined in the following paragraphs.

When the simulation was originally created, somewhat arbitrary values were entered for the TYPE 8 controller proportional and integral gains, $\mathrm{K}_{\mathrm{p}}$ and $\mathrm{K}_{\mathrm{i}}$. These values were intentionally too low, roughly an order of magnitude below the final values, to ensure a stable response. The simulation was then run for 3600 seconds, using a boundary file containing a constant value of the controller setpoint $\mathrm{C}_{4}$ (as in the first six 1 ines of Listing 6.2-3) to allow the system to reach steady state. The final state file, INITOUT.DAT, was then renamed INITIN.DAT for use as an initial state file.

Using the initialization option, and the boundary file shown in Listing 6.2-3, a series of runs was made from the same initial steady state. Before each run, HVACGEN was used to modify the controller gains. This procedure was repeated until a satisfactory response was obtained.

To emphasize the value of the initialization feature, the simulation was run again without the initialization option, using the final controller gains and a constant controller setpoint for 3600 seconds. This simulation ran at the ainimum time step of 0.1 second for the first 88 steps, reached or exceeded a simulation time of 60 seconds on the 129 th step, and ended at 3600 seconds on the 167 th step. (Somewhat different results might be obtained when the program is run on a different computer, since the time step size does depend to some extent on machine precision.) The final state of this run second initialization, shown in Listing 6.2-4, was used to initialize one last run. Listing 6.2-5 shows the beginning of the output file from this last simulation, the results of which are plotted in Figure 6.2-3.

\subsubsection{Interpretation}

If this example is used to verify that a newly installed version of HVACSIM H $^{+}$ is operating correctly, the following steps are recommended.

1. Run HVACGEN and enter the information in listing 6.2-1 to create the simulation work file. The output of HVACGEN should be identical to listing $6.2-2$.

2. Run SLIMCON to create the model definition file (not shown). 
3. Create a boundary variable file (default name BOUNDARY.DAT) consisting only of the first six lines of listing 6.2-3.

4. Run HVACSIM with a minimum time step of 0.1 second, a maximum time step of 1800 seconds, and a stopping time of 3600 seconds. Do not read an initialization file.

5. Compare the final state file (default name INITOUT.DAT) with listing 6.24. Lines 2-8 of this file give the final values of the 33 state variables, in the same order as the list of variable initial values in 1 isting 6.2-1. These numbers should all agree within the relative error tolerance of half a percent. Lines 9 and 10 consist of 33 integers: -1 if the final value of a variable is less than its next to last value, and +1 otherwise. In other words, these integers represent the sign of the derivative of each variable, whether or not the variable is involved in a differential equation. Differences here are unlikely but probably not important. The remaining lines of the file contain values stored in the SAVED workspace vector, and are not easily interpreted. Some of the numbers (such as the eighth one) are proportional to the length of the last time step of the simulation, and may be expected to vary from one computer to another. Differences in this set of numbers need not concern the user.

6. Copy or rename the final state file for use as an initial state file (default name INITIN.DAT), and prepare a boundary file identical to listing 6.2-3. Run the simulation as before, except with a stopping time of 1800 seconds and with the initialization option. The results should be within half a percent of the values in listing 6.2-5, though a direct comparison may be difficult if the time steps differ. It may be helpful to compare a graph of the coil outlet air temperature with figure $6.2-3$ if graphics software (or graph paper) is available to the user. 
TYPICAL PIPING ARRANGEMENT FOR CONSTANT FLOW, VARIABLE TEMPERATURE CONTROL LOOKS LIKE THIS:

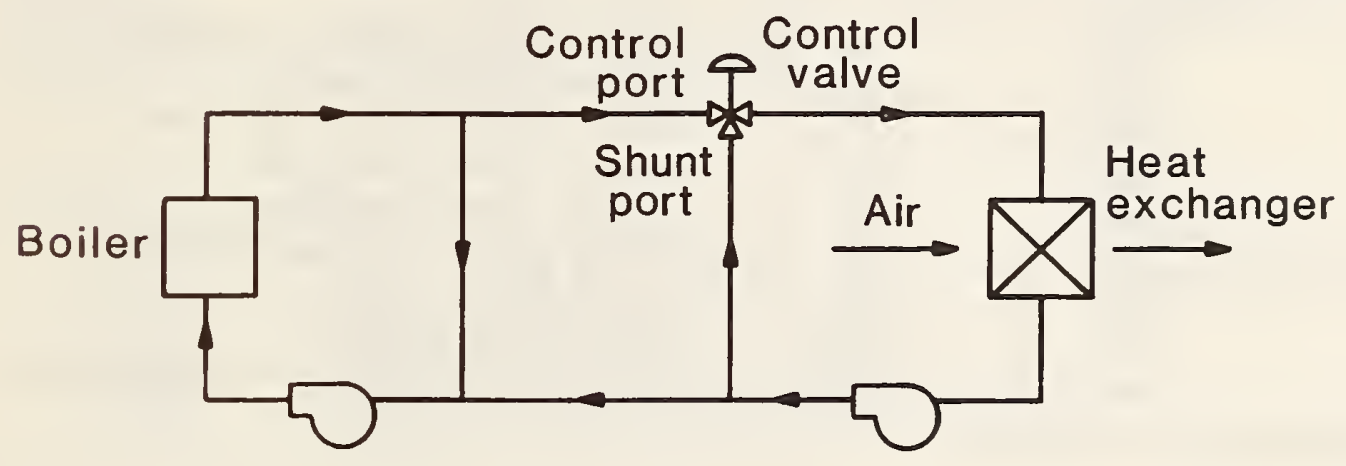

igure 6.2-1. Constant flow, variable temperature control system

HEATING COIL CONTROL LOOP, CONSTANT FLOW, VARIABLE TEMPERATURE CONTROL

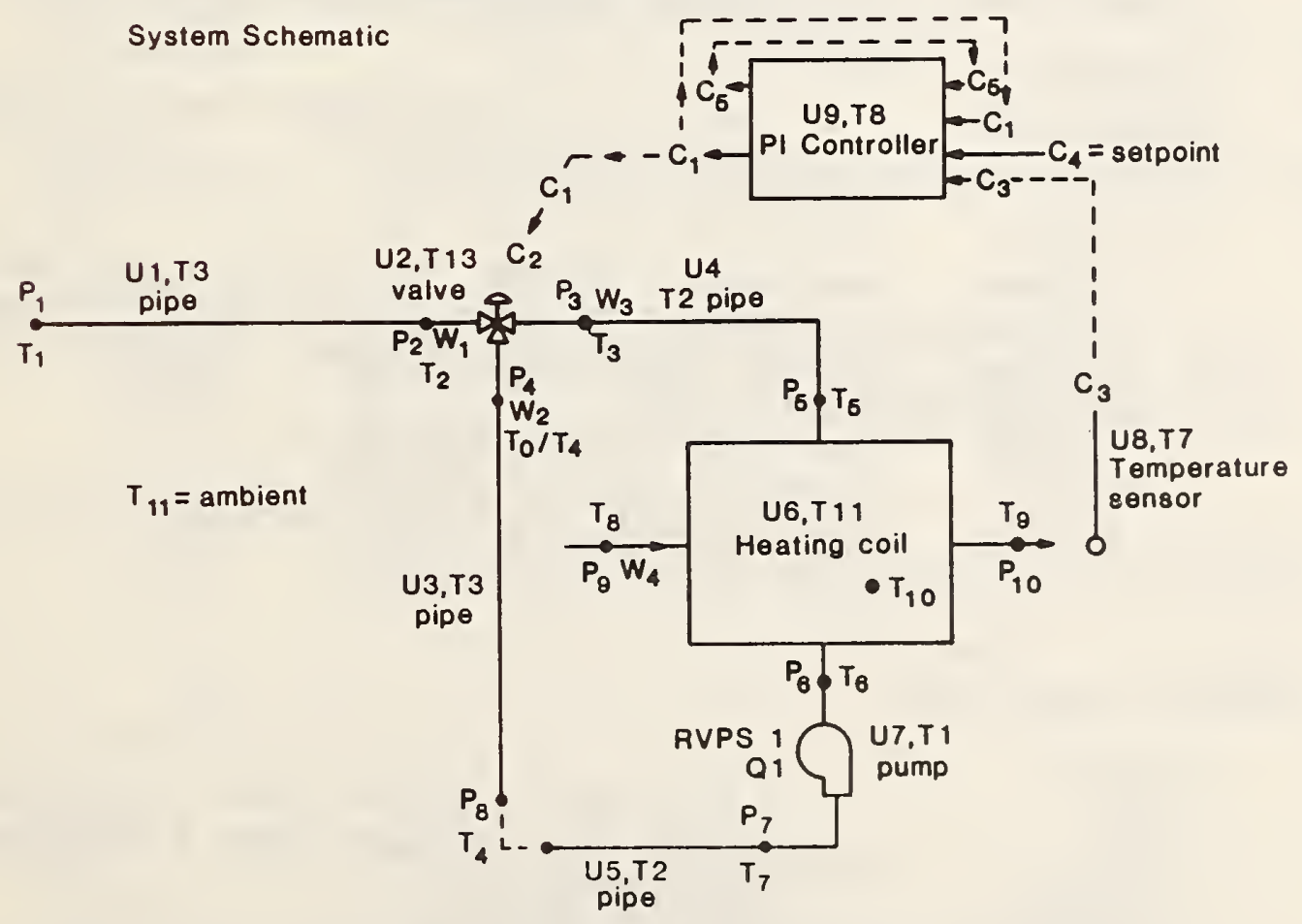

Figure 6.2-2. System schematic for heating coil control luop 
Listing 6.2-1. Summary of EXAMPLE2 Simulation Configuration.

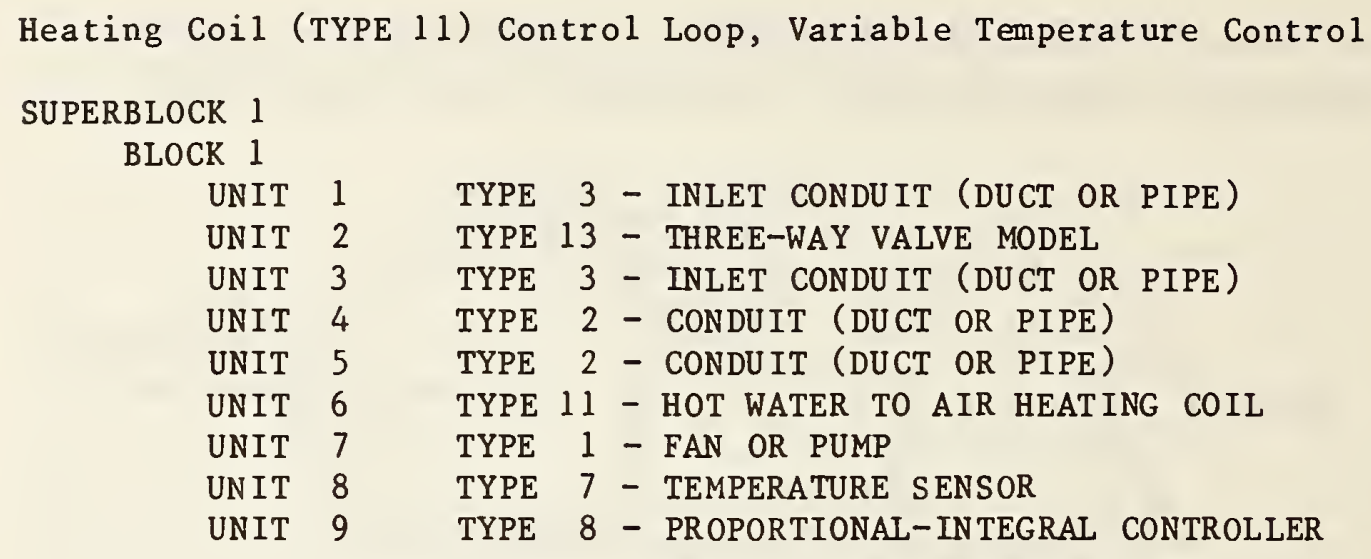

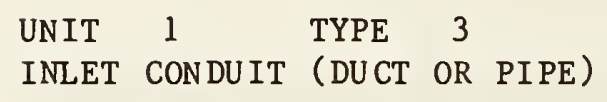

1 IN PUTS :

PRESSURE 1 - INLET FLUID PRESSURE

PRESSURE 2 - OUTLET FLUID PRESSURE

TEMPERATURE 1 - INLET FLUID TEMPERATURE

TEMPERATURE 11 - AMBIENT AIR TEMPERATURE

TEMPERATURE 2 - OUTLET FLUID TEMPERATURE (SAME AS FIRST OUTPUT)

2 OUTPUTS :

TEMPERATURE 2 - OUTLET FLUID TEMPERATURE (SAME AS FIFTH INPUT)

FLOW

1 - FLUID MASS FLOW RATE

3 PARAMETERS :

3.80000 INSIDE HEAT TRANSFER COEFFICIENT X AREA (KW/C)

$0.440000 \mathrm{E}-02$ OUTSIDE HEAT TRANSFER COEFFICIENT X AREA (KW/C)

3.22000 THERMAL CAPACITANCE OF CONDUIT MATERIAL (KJ/C)

$0.355000 \mathrm{E}-02$ VOLUME (M3)

5.00000 FLOW RESISTANCE [0.001/(KG M)]

0.000000 HEIGHT OF OUTLET ABOVE INLET (M)

2.00000 MODE: 2=WATER, 1=AIR, NEG.=DETAILED, POS.=SIMPLE DYNAMI

$\begin{array}{llll}\text { UNIT } 2 \text { TYPE } 13 & 13\end{array}$

THREE-WAY VALVE MODEL

1 IN PUTS :

FLOW

FLOW

PRESSURE

TEMPERATURE

TEMPERATURE

1 - INLET MASS FLOW RATE, PORT 1 (OPEN WHEN CONTROL $=1$

2 - INLET MASS FLOW RATE, PORT 2 (OPEN WHEN CONTROL $=0$

3 - OUTLET PRESSURE

2 - INLET TEMPERATURE, PORT 1

4 - INLET TEMPERATURE; PORT 2 
CONTROL

CONTROL

2 OUTPUTS :

CONTROL

FLOW

PRESSURE

PRESSURE

TEMPERATURE

CONTROL
1 - CONTROL VARIABLE FROM CONTROLLER

2 - ACTUATOR RELATIVE POSITION (SAME AS OUTPUT 1)

2 - ACTUATOR RELATIVE POSITION

3 - OUTLET MASS FLOWRATE

2 - INLET PRESS., PORT 1 (OPEN $\Rightarrow$ CONTROL VARIABLE = 1)

4 - INLET PRESS., PORT 2 (OPEN $\Rightarrow$ CONTROL VARIABLE $=0$ )

3 - OUTLET TEMPERATURE

6 - VALVE STEM RELATIVE POSITION

3 PARAMETERS:

0.300000 PORT 1 FLOW RESISTANCE, PORT 1 OPEN (CTRL=1) $[0.001 /(\mathrm{KG}$

1.50000 PORT 2 FLOW RESISTANCE, PORT 2 OPEN (CTRL=0) $[0.001 /(\mathrm{KG}$

$0.400000 \mathrm{E}-02$ LEAKAGE PARAMETER (DIMENSIONLESS)

10.0000 ACTUATOR TIME CONSTANT (SEC)

0.100000 HYSTERESIS PARAMETER (DIMENSIONLESS)

1.00000 PORTS $1,2 \mathrm{MODE}: 0=>\mathrm{LIN} / \mathrm{LIN}, 1=>\mathrm{EXP} / \mathrm{LIN}, \mathrm{ELSE}=>\mathrm{EXP} / \mathrm{EXP}$

$\begin{array}{llll}\text { UNIT } & 3 \text { TYPE } 3\end{array}$

INLET CONDUIT (DUCT OR PIPE)

1 INPUTS :

PRESSURE

PRESSURE

TEMPERATURE

TEMPERATURE

TEMPERATURE

2 OUTPUTS :

TEMPERATURE FLOW
8 - INLET FLUID PRESSURE

4 - OUTLET FLUID PRESSURE

4 - INLET FLUID TEMPERATURE

11 - AMBIENT AIR TEMPERATURE

4 - OUTLET FLUID TEMPERATURE (SAME AS FIRST OUTPUT)

0 - OUTLET FLUID TEMPERATURE (SAME AS FIFTH INPUT)

2 - FLUID MASS FLOW RATE

3 PARAMETERS:

0.000000

0.000000

0.000000

0.000000

14.8000

0.558800

2.00000

INSIDE HEAT TRANSFER COEFFICIENT X AREA (KW/C)

OUTSIDE HEAT TRANSFER COEFFICIENT X AREA (KW/C)

THERMAL CAPACITANCE OF CONDUIT MATERIAL (KJ/C)

VOLUME (M3)

FLOW RESISTANCE [0.001/(KG M)]

HE IGHT OF OUTLET ABOVE INLET (M)

MODE : 2=WATER, 1=AIR, NEG.=DETAILED, POS .=SIMPLE DYNAMI

UNIT 4 TYPE 2

CONDUIT (DUCT OR PIPE)

1 IN PUTS :

FLOW

PRESSURE

3 - FLUID MASS FLOW RATE

TEMPERATURE

5 - OUTLET PRESSURE

3 - FLUID INLET TEMPERATURE

TEMPERATURE

11 - AMBIENT TEMPERATURE 


\section{TEMPERATURE 5 - OUTLET FLUID TEMPERATURE (SAME AS FIRST OUTPUT)}

2 OUTPUTS :

TEMPERATURE 5 - OUTLET FLUID TEMPERATURE (SAME AS FIFTH INPUT)

PRESSURE 3 - INLET PRESSURE

3 PARAMETERS :

2.97000 INSIDE HEAT TRANSFER COEFFICIENT X AREA (KW/C)

$0.310000 \mathrm{E}-02$ OUTSIDE HEAT TRANSFER COEFFICIENT X AREA (KW/C)

2.27700 THERMAL CAPACITANCE OF CONDUIT MATERIAL (KJ/C)

$0.251000 \mathrm{E}-02$ VOLUME (M3)

14.3600 FLOW RESISTANCE [0.001/(KG M)]

-0.558800 HE IGHT OF OUTLET ABOVE INLET (M)

2.00000 MODE : 2=WATER, l=AIR, NEG.=DETAILED, POS $=$ SIMPLE DYNAMI

UNIT 5 TYPE 2

CONDUIT (DUCT OR PIPE)

1 IN PUTS :

FLOW

PRESSURE

TEMPERATURE

TEMPERATURE

TEMPERATURE

3 - FLUID MASS FLOW RATE

8 - OUTLET PRESSURE

7 - FLUID INLET TEMPERATURE

11 - AMBIENT TEMPERATURE

4 - OUTLET FLUID TEMPERATURE (SAME AS FIRST OUTPUT)

2 OUTPUTS :

TEMPERATURE

PRESSURE

4 - OUTLET FLUID TEMPERATURE (SAME AS FIFTH INPUT)

7 - INLET PRESSURE

3 PARAME TERS :

2.70000 INSIDE HEAT TRANSFER COEFFICIENT X AREA (KW/C)

$0.281000 \mathrm{E}-02$ OUTSIDE HEAT TRANSFER COEFFICIENT X AREA (KW/C)

2.07000 THERMAL CAPACITANCE OF CONDUIT MATERIAL (KJ/C)

$0.227800 \mathrm{E}-02$ VOLUME (M3)

11.9000 FLOW RESISTANCE $[0.001 /(\mathrm{KG}$ M) $]$

0.000000 HEIGHT OF OUTLET ABOVE INLET (M)

2.00000 MODE : 2=WATER, I=AIR, NEG.=DETAILED, POS.=SIMPLE DYNAMI

UNIT 6 TYPE 11

HOT WATER TO AIR HEATING COIL

1 IN PUTS :

PRESSURE

FLOW

TEMPERATURE

TEMPERATURE

PRESSURE

FLOW

TEMPERATURE

TEMPERATURE
10 - OUTLET AIR PRESSURE

4 - AIR MASS FLOW RATE

8 - INLET AIR TEMPERATURE

9 - OUTLET AIR TEMPERATURE (SAME AS FIRST OUTPUT)

6 - OUTLET WATER PRESSURE

3 - WATER MASS FLOW RATE

5 - INLET WATER TEMPERATURE

6 - OUTLET WATER TEMPERATURE (SAME AS SECOND OUTPUT) 
TEMPERATURE 10 - DUMM TEMPERATURE (SAME AS THIRD OUTPUT)

2 OUTPUTS :

TEMPERATURE 9 - OUTLET AIR TEMPERATURE

TEMPERATURE 6 - OUTLET WATER TEMPERATURE

TEMPERATURE 10 - DUMMY TEMP. (USED INTERNALLY FOR AIR TEMP. DYNAMICS

PRESSURE 9 - INLET AIR PRESSURE

PRESSURE $\quad 5$ - INLET WATER PRESSURE

3 PARAMETERS :

1.73610 COEFFICIENT FOR AIR SIDE HEAT TRANSFER CORRELATION ( -

0.448735 EXPONENT FOR AIR SIDE HEAT TRANSFER CORRELATION ( - )

14.1111 COEFFICIENT FOR WATER SIDE HEAT TRANSFER CORRELATION (

1.13024 EXPONENT FOR WATER SIDE HEAT TRANSFER CORRELATION ( - )

0.120000 AIR FLOW RESISTANCE PARAMETER [0.001/(KG M)]

7.00000 WATER FLOW RESISTANCE PARAMETER [0.001/(KG M)]

$0.730000 E-02$ WATER SIDE VOLUME OF COIL (M3)

26.2000 CAPACITANCE (MASS X SPECIFIC HEAT) OF COIL MATERIAL (KJ

$\begin{array}{llll}\text { UNIT } & 7 & \text { TYPE } & 1\end{array}$

FAN OR PUMP

1 INPUTS :

FLOW

3 - MASS FLOW RATE OF FLUID

PRESSURE

7 - OUTLET PRESSURE

RVPS

TEMPERATURE

1 - FAN OR PUMP ROTATIONAL SPEED

6 - INLET FLUID TEMPERATURE

2 OUTPUTS :

PRESSURE

TEMPERATURE

6 - INLET PRESSURE

7 - OUTLET FLUID TEMPERATURE

POWER

1 - POWER CONSUMPTION

3 PARAMETERS :

1.65000 1ST PRESSURE COEFFICIENT

-70.0000 2ND PRESSURE COEFFICIENT

5.00000 3RD PRESSURE COEFFICIENT

0.000000 4TH PRESSURE COEFFICIENT

30.0000 5TH PRESSURE COEFFICIENT

0.950000 1ST EFFICIENCY COEFFICIENT

0.000000 2ND EFFICIENCY COEFFICIENT

0.000000 3RD EFFICIENCY COEFFICIENT

0.000000 4TH EFFICIENCY COEFFICIENT

0.000000 5TH EFFICIENCY COEFFICIENT

0.127000 DIAMETER (M)

2.00000 MODE: $A I R=1$, WATER $=2$

$\begin{array}{llll}\text { UNIT } & 8 & \text { TYPE } & 7\end{array}$

TEMPERATURE SENSOR 
INPUTS :

TEMPERATURE 9 - INPUT TEMPERATURE

CONTROL

3 - SENSOR OUTPUT (MODIFIED BY GAIN AND OFFSET)

2 OUTPUTS :

CONTROL

3 - SENSOR OUTPUT (MODIFIED BY GAIN AND OFFSET)

3

PARAMETERS :

20.0000

0.000000

100.000

SENSOR TIME CONSTANT (SEC)

TEMPERATURE OFFSET (C)

TEMPERATURE GAIN (C)

$\begin{array}{llll}\text { UNIT } & 9 & \text { TYPE } & 8\end{array}$

PROPORT IONAL-INTEGRAL CONTROLLER

1 INPUTS :

CONTROL

CONTROL

CONTROL

CONTROL

3 - CONTROLLED VARIABLE

4 - SET POINT FOR CONTROLLED VARIABLE

5 - INTEGRAL PORTION OF CTRL SIGNAL (SAME AS IST OUTPUT

1 - OUTPUT CONTROL SIGNAL (SAME AS SECOND OUTPUT)

2 OUTPUTS :

CONTROL 5 - INTEGRAL PORTION OF CONTROL SIGNAL

CONTROL

1 - OUTPUT CONTROL SIGNAL

3 PARAMETERS:

2.00000 PROPORTIONAL GAIN (DIMENSIONLESS)

0.200000 E-01 INTEGRAL GAIN (DIMENSIONLESS)

2.00000 CONTROLLER TIME CONSTANT (SEC)

Initial Variable Values:

$\begin{array}{lrrr}\text { PRESSURE } & 1 \rightarrow & 15.0000 & (\mathrm{KPa}) \\ \text { PRESSURE } & 2 \rightarrow & 10.0000 & (\mathrm{KPa}) \\ \text { PRESSURE } & 3 \rightarrow & -15.0000 & (\mathrm{KPa}) \\ \text { PRESSURE } & 4 \rightarrow & -15.0000 & (\mathrm{KPa}) \\ \text { PRESSURE } & 5 \rightarrow & -32.0000 & (\mathrm{KPa}) \\ \text { PRESSURE } & 6 \rightarrow & -40.0000 & (\mathrm{KPa}) \\ \text { PRESSURE } & 7 \rightarrow & 15.0000 & (\mathrm{KPa}) \\ \text { PRESSURE } & 8 \rightarrow & 0.000000 & (\mathrm{KPa}) \\ \text { PRESSURE } & 9 \rightarrow & 0.700000 & (\mathrm{KPa}) \\ \text { PRESSURE } & 10 \rightarrow & 0.000000 & (\mathrm{KPa}) \\ \text { FLOW } & 1 \rightarrow & 0.400000 \mathrm{E} & \mathrm{(Kg} / \mathrm{s}) \\ \text { FLOW } & 2 \rightarrow & 1.00000 & (\mathrm{Kg} / \mathrm{s}) \\ \text { FLOW } & 3 \rightarrow & 1.04000 & (\mathrm{Kg} / \mathrm{s}) \\ \text { FLOW } & 4 \rightarrow & 2.35000 & (\mathrm{Kg} / \mathrm{s}) \\ \text { TEMPERATURE } & 1 \rightarrow & 70.0000 & (\mathrm{C}) \\ \text { TEMPERATURE } & 2 \rightarrow & 70.0000 & (\mathrm{C}) \\ \text { TEMPERATURE } & 3 \rightarrow & 26.5000 & (\mathrm{C}) \\ \text { TEMPERATURE } & 4 \rightarrow & 24.0000 & (\mathrm{C}) \\ \text { TEMPERATURE } & 5 \rightarrow & 26.5000 & (\mathrm{C})\end{array}$




\begin{tabular}{lrrl} 
TEMPERATURE & $6 \rightarrow$ & 24.0000 & (C) \\
TEMPERATURE & $7 \rightarrow$ & 24.0000 & (C) \\
TEMPERATURE & $8 \rightarrow$ & 0.000000 & (C) \\
TEMPERATURE & $9 \rightarrow$ & 18.0000 & (C) \\
TEMPERATURE & $10 \rightarrow$ & 20.0000 & (C) \\
TEMPERATURE & $11 \rightarrow$ & 35.0000 & (C) \\
CONTROL & $1 \rightarrow$ & 0.400000 & (DIMENS IONLESS) \\
CONTROL & $2 \rightarrow$ & 0.400000 & (DIMENSIONLESS) \\
CONTROL & $3 \rightarrow$ & 0.180000 & (DIMENSIONLESS) \\
CONTROL & $4 \rightarrow$ & 0.200000 & (DIMENSIONLESS) \\
CONTROL & $5 \rightarrow$ & 0.000000 & (DIMENSIONLESS) \\
CONTROL & $6 \rightarrow$ & 0.000000 & (DIMENSIONLESS) \\
RVPS & $1 \rightarrow$ & 57.5000 & (Rev/S) \\
POWER & $1 \rightarrow$ & 0.100000 & (kW) \\
\hline
\end{tabular}

Simulation Error Tolerances:

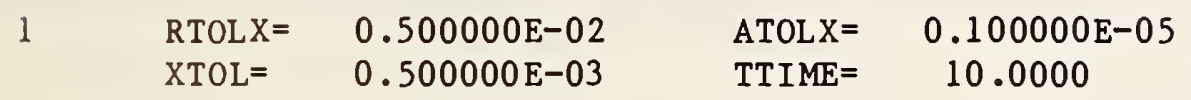

SUPERBLOCK 1

2 FREEZE OPTION 0 SCAN OPTION 0

The following are Boundary Variables in the simulation:

CONTROL 4

The following are the reported variables:

\begin{tabular}{|c|c|}
\hline SUPERBLOCK 1 & REPORTING INTERVAL \\
\hline FLOW & 3 \\
\hline FLOW & 1 \\
\hline TEMPERATURE & 9 \\
\hline CONTROL & 3 \\
\hline CONTROL & 1 \\
\hline CONTROL & 5 \\
\hline CONTROL & 2 \\
\hline CONTROL & 4 \\
\hline
\end{tabular}




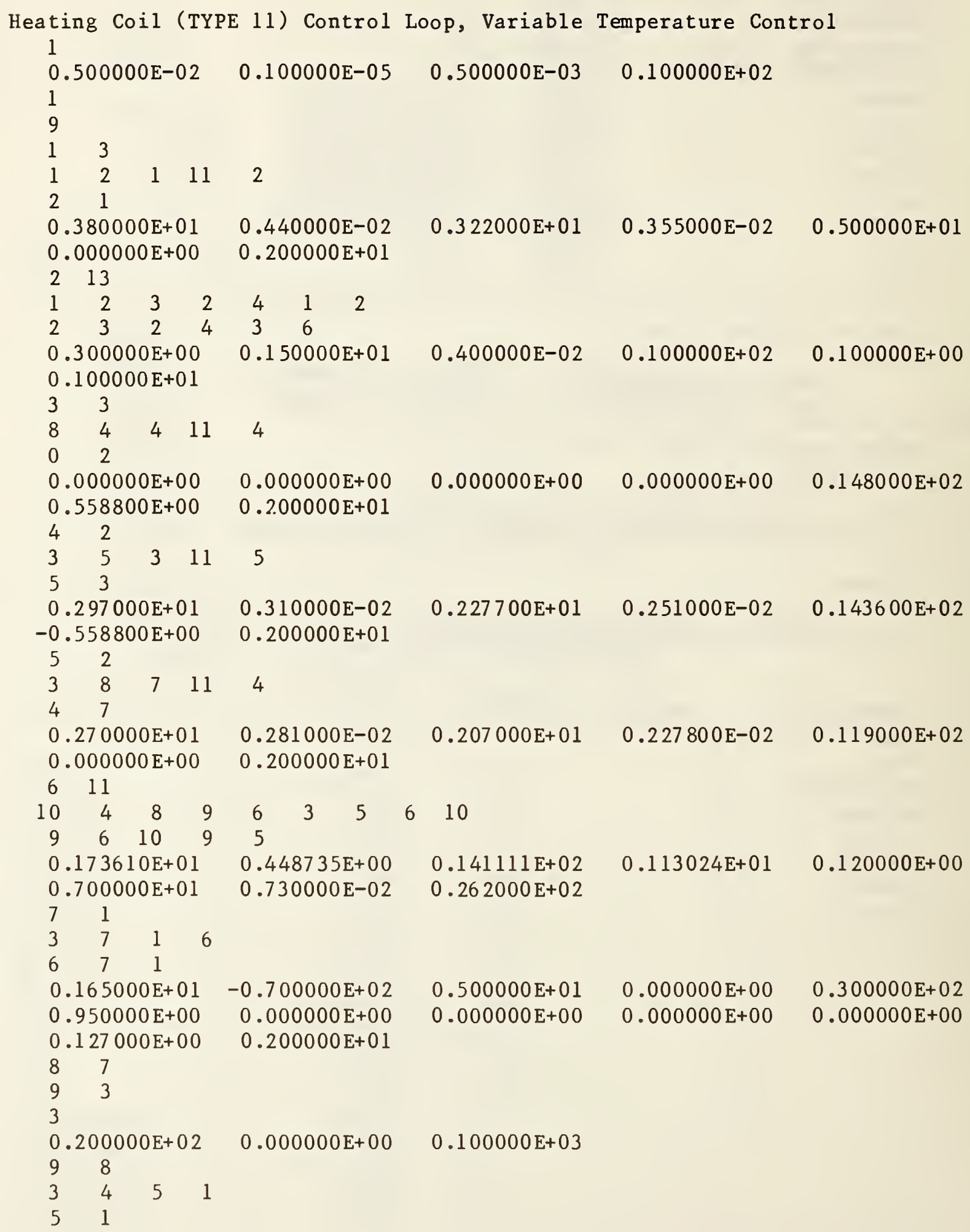




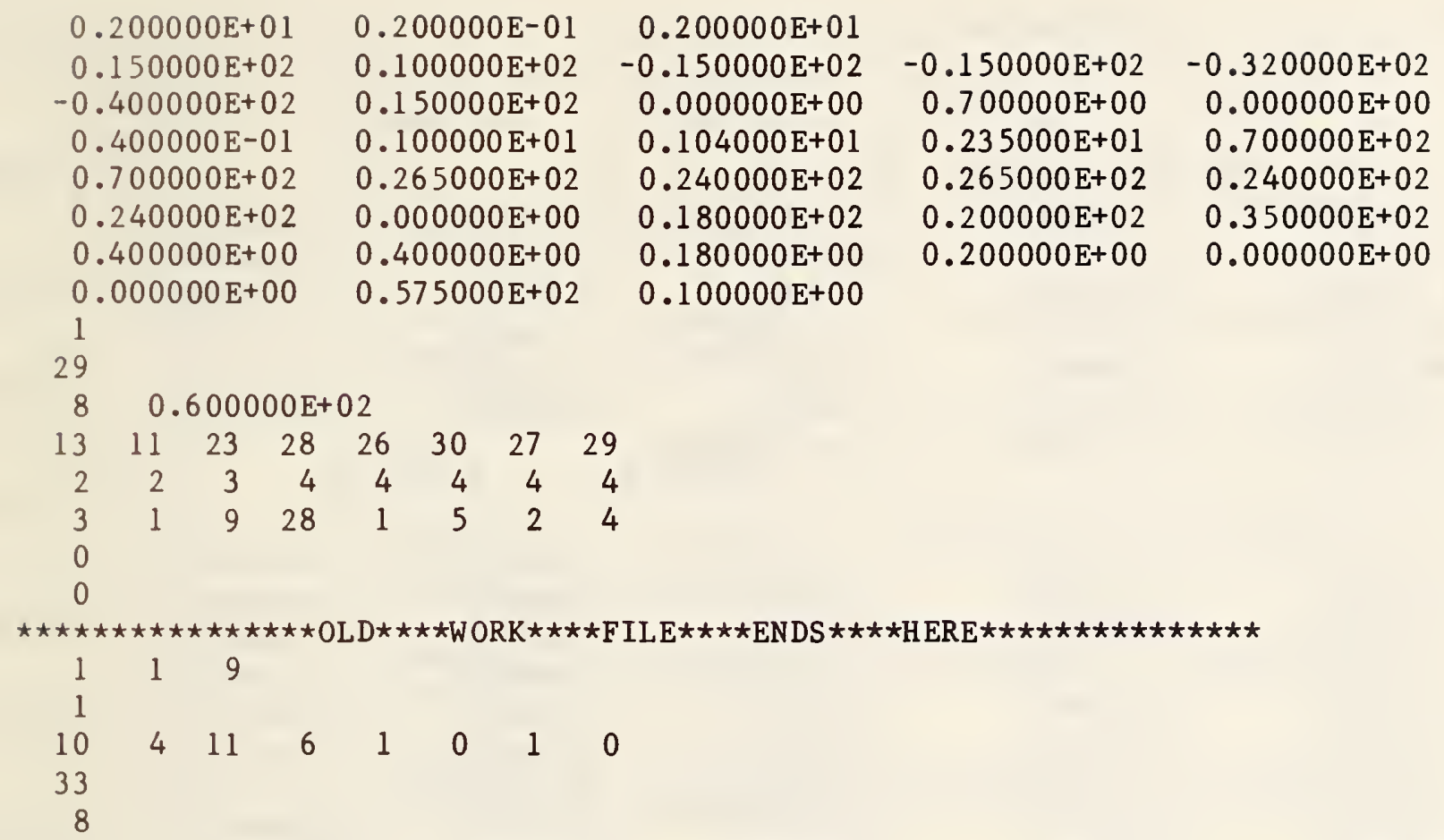

$0.100000 \mathrm{E}+00$

29

$80.600000 \mathrm{E}+02$

$\begin{array}{rrrrr}13 & 11 & 23 & 28 & 26 \\ 2 & 2 & 3 & 4 & 4\end{array}$

$\begin{array}{llllllll}3 & 1 & 9 & 28 & 1 & 5 & 2 & 4\end{array}$

0

0

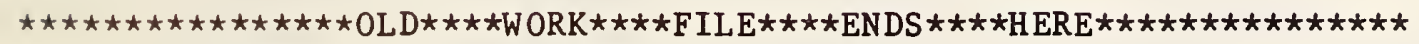

Listing 6.2-3. Boundary Variable File for Example 2.
$0 . \quad 0.20$
$100 ., \quad 0.20$
$200 \ldots, 0.20$
$300 ., \quad 0.20$
$400 \ldots, 0.20$
$500 ., 0.20$
$600,0.20$
$600 \ldots .25$
$800 \ldots, 0.25$
$1000 \ldots 0.25$
$1200 \ldots \quad 0.25$
$1200 \ldots 0.15$
$1400 ., 0.15$
$1600 \ldots 0.15$
$1800 \ldots 0.15$
2400 ., 0.15
2800 ., 0.15
3200.0 .15
$3600,0.15$ 


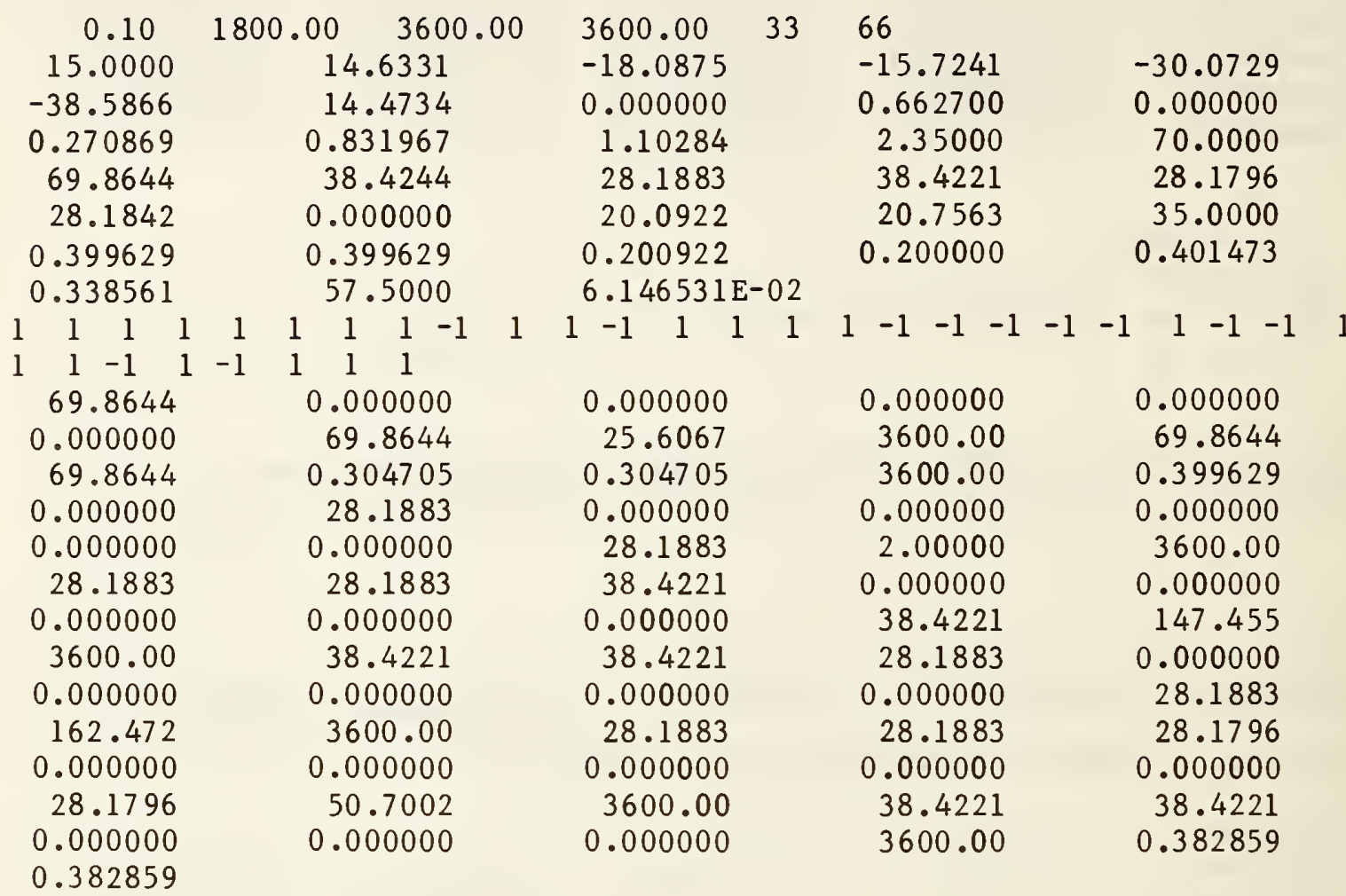

Listing 6.2-5. A Portion of the Simulation Output File, MODOUT.DAT

\begin{tabular}{|c|c|c|c|c|}
\hline SUPERBLOCK l & 0.10 & & & \\
\hline 1.10284 & 0.270869 & 20.0922 & 0.200922 & 0.399629 \\
\hline 0.401473 & 0.399629 & 0.200000 & & \\
\hline SUPERBLOCK 1 & 0.30 & & & \\
\hline 1.10284 & 0.270869 & 20.0922 & 0.200922 & 0.399629 \\
\hline 0.401473 & 0.399629 & 0.200000 & & \\
\hline SUPERBLOCK 1 & 0.70 & & & \\
\hline 1.10284 & 0.270869 & 20.0922 & 0.200922 & 0.399629 \\
\hline 0.401473 & 0.399629 & 0.200000 & & \\
\hline SUPERBLOCK 1 & 1.50 & & & \\
\hline 1.10284 & 0.270869 & 20.0922 & 0.200922 & 0.399629 \\
\hline 0.401473 & 0.399629 & 0.200000 & & \\
\hline SUPERBLOCK 1 & 3.10 & & & \\
\hline 1.10284 & 0.270869 & 20.0922 & 0.200922 & 0.399629 \\
\hline 0.401473 & 0.399629 & 0.200000 & & \\
\hline SUPERBLOCK 1 & 6.30 & & & \\
\hline 1.10284 & 0.270869 & 20.0922 & 0.200922 & 0.399629 \\
\hline 0.401473 & 0.399629 & 0.200000 & & \\
\hline
\end{tabular}


0.401473

0.399629

25.50

1.10284

0.270869

0.399629

0.401473

SUPERBLOCK 1

51.10

1.10284

0.270869

0.399630

0.401473

SUPERBLOCK 1

1.10284

0.401473

SUPERBLOCK 1

1.10284

0.401473

SUPERBLOCK 1

1.10284

0.401473

SUPERBLOCK 1

1.10284

0.401473

SUPERBLOCK 1

1.10284

0.401571

SUPERBLOCK 1

1.10284

0.401669

SUPERBLOCK 1

1.10284

0.401767

SU PERBLOCK 1

$$
1.10284
$$

0.401866

SUPERBLOCK 1

1.10284

0.401964

SUPERBLOCK 1

1.10284

0.402062

SUPERBLOCK 1

1.10284

0.402160

SUPERBLOCK 1

1.10284

0.402258

SUPERBLOCK 1

1.10284

0.402357

SUPERBLOCK 1

1.10284

0.402455

102.30

0.270869

0.399630

204.70

0.270869

0.399630

409.50

0.270869

0.399630

600.00

0.270869

0.399630

600.10

0.270869

0.399677

600.20

0.270869

0.399769

600.30

0.270869

0.399902

600.40

0.270869

0.400076

600.50

0.270869

0.400286

600.60

0.270869

0.400532

600.70

0.270869

0.400811

600.80

0.270869

0.401120

600.90

0.270869

0.401459

601.00

0.270869

0.401826
20.0922

0.200000

20.0921

0.200000

20.0921

0.200000

20.0921

0.200000

20.0921

0.200000

20.0921

0.200000

20.0921

0.200000

20.0921

0.250000

0.200922

0.404396

20.0921

0.200922

0.408941

0.250000

20.0921

0.250000

0.200922

0.413273

20.0921

0.250000

0.200922

0.417404

20.0921

0.250000

0.200922

0.421343

20.0921

0.200922

0.425099

0.250000

20.0921

0.250000

0.200922

0.428681

20.0921

0.250000

0.200922

0.432097

20.0921

0.250000

20.0921

0.250000
0.200922

0.435355

0.200922

0.438462 


\begin{tabular}{|c|c|c|c|c|}
\hline SUPERBLOCK 1 & 601.10 & & & \\
\hline 1.10284 & 0.270869 & 20.0921 & 0.200922 & 0.441426 \\
\hline 0.402553 & 0.402218 & 0.250000 & & \\
\hline SUPERBLOCK 1 & 601.20 & & & \\
\hline 1.10284 & 0.270869 & 20.0921 & 0.200922 & 0.444254 \\
\hline 0.402651 & 0.402634 & 0.250000 & & \\
\hline SUPERBLOCK 1 & 601.30 & & & \\
\hline 1.10284 & 0.270869 & 20.0921 & 0.200922 & 0.446952 \\
\hline 0.402749 & 0.403073 & 0.250000 & & \\
\hline SUPERBLOCK 1 & 601.60 & & & \\
\hline 1.10284 & 0.270869 & 20.0921 & 0.200922 & 0.454429 \\
\hline 0.403044 & 0.404493 & 0.250000 & & \\
\hline SUPERBLOCK 1 & 601.80 & & & \\
\hline 1.10307 & 0.272121 & 20.0921 & 0.200922 & 0.458890 \\
\hline 0.403240 & 0.405526 & 0.250000 & & \\
\hline SUPERBLOCK 1 & 602.40 & & & \\
\hline 1.10405 & 0.277399 & 20.0918 & 0.200922 & 0.470092 \\
\hline 0.403829 & 0.408950 & 0.250000 & & \\
\hline SUPERBLOCK 1 & 602.90 & & & \\
\hline 1.10496 & 0.282310 & 20.0913 & 0.200921 & 0.477331 \\
\hline 0.404320 & 0.412085 & 0.250000 & & \\
\hline SUPERBLOCK 1 & 603.80 & & & \\
\hline 1.10674 & 0.291979 & 20.0901 & 0.200921 & 0.486688 \\
\hline 0.405203 & 0.418118 & 0.250000 & & \\
\hline SUPERBLOCK 1 & 604.90 & & & \\
\hline 1.10906 & 0.304551 & 20.0908 & 0.200920 & 0.493879 \\
\hline 0.406283 & 0.425706 & 0.250000 & & \\
\hline SUPERBLOCK 1 & 606.30 & & & \\
\hline 1.11203 & 0.320768 & 20.1067 & 0.200924 & 0.499292 \\
\hline 0.407657 & 0.435095 & 0.250000 & & \\
\hline SUPERBLOCK 1 & 607.40 & & & \\
\hline 1.11429 & 0.333154 & 20.1442 & 0.200942 & 0.502094 \\
\hline 0.408737 & 0.441996 & 0.250000 & & \\
\hline SUPERBLOCK 1 & 608.50 & & & \\
\hline 1.11645 & 0.345027 & 20.2108 & 0.200987 & 0.504228 \\
\hline 0.409815 & 0.448405 & 0.250000 & & \\
\hline SUPERBLOCK 1 & 609.80 & & & \\
\hline 1.11885 & 0.358309 & 20.3266 & 0.201097 & 0.506236 \\
\hline 0.411088 & 0.455356 & 0.250000 & & \\
\hline SUPERBLOCK 1 & 611.80 & & & \\
\hline 1.12222 & 0.377102 & 20.5704 & 0.201427 & 0.508469 \\
\hline 0.413037 & 0.464827 & 0.250000 & & \\
\hline SUPERBLOCK 1 & 614.10 & & & \\
\hline 1.12560 & 0.396129 & 20.9591 & 0.202119 & 0.510071 \\
\hline 0.415253 & 0.474024 & 0.250000 & & \\
\hline SUPERBLOCK 1 & 616.00 & & & \\
\hline 1.12800 & 0.409695 & 21.3225 & 0.202968 & 0.510734 \\
\hline 0.417053 & 0.480363 & 0.250000 & & \\
\hline SUPERBLOCK 1 & 617.40 & & & \\
\hline 1.12953 & 0.418431 & 21.5953 & 0.203754 & 0.510867 \\
\hline 0.418358 & 0.484353 & 0.250000 & & \\
\hline
\end{tabular}


SUPERBLOCK 1

1.13096

0.419731

SUPERBLOCK 1

1.13255

0.421512

SUPERBLOCK 1

1.13396

0.423481

SUPERBLOCK 1

1.13490

0.425274

SUPERBLOCK 1

1.13546

0.426818

SUPERBLOCK 1

1.13546

0.429176

SUPERBLOCK 1

1.13546

0.430981

SUPERBLOCK 1

1.13546

0.432767

SUPERBLOCK 1

1.13546

0.435131

SUPERBLOCK 1

1.13546

0.436956

SU PERBLOCK 1

1.13546

0.438893

SUPERBLOCK 1

1.13546

0.439789

SUPERBLOCK 1

1.13546

0.438771

SU PERBLOCK 1

1.13546

0.435672

SUPERBLOCK 1

1.13546

0.430133

SUPERBLOCK 1

1.13377

0.420616

SUPERBLOCK 1

1.13103

0.408619
618.90

$$
\begin{aligned}
& 0.426632 \\
& 0.488039
\end{aligned}
$$

620.90

$$
0.435782
$$

0.492085

623.20

$$
0.443899
$$

0.495617

625.40

0.449391

0.497978

627.40

0.452618

0.499355

630.70

0.454850

0.500186

633.50

0.454850

0.499636

636.60

0.454850

0.497887

641.50

0.454850

0.493135

646.40

0.454850

0.486695

654.40

0.454850

0.474434

668.70

0.454850

0.452516

680.50

0.454850

0.437651

698.40

0.454850

0.421290

721.60

0.454850

0.407418

758.00

0.442817

0.395148

811.10

0.427015

0.388210
21.8777

0.250000

22.2321

0.250000

22.6437

0.250000

23.0461

0.250000

23.3938

0.250000

23.9221

0.250000

24.3167

0.250000

24.6884

0.250000

25.1423

0.250000

25.4643

0.250000

25.8194

0.250000

26.1914

0.250000

26.3548

0.250000

26.4294

0.250000

26.4245

0.250000

26.2428

0.250000

25.8132

0.250000
0.204737

0.510695

0.206245

0.509989

0.208222

0.508555

0.210335

0.506611

0.212413

0.504405

0.216082

0.499985

0.219347

0.495614

0.223017

0.490311

0.228728

0.481378

0.234061

0.472406

0.241593

0.458813

0.251424

0.439169

0.256622

0.427235

0.261274

0.414233

0.263788

0.403222

0.263768

0.393371

0.260242

0.388128 
SU PERBLOCK 1

1.12996

0.397847

SUPERBLOCK 1

1.12843

0.387409

SUPERBLOCK 1

1.12775

0.382850
895.30
0.420867
0.385453
1063.60
0.412160
0.381488
1200.00

0.408268

0.379701

$\begin{array}{rll}25.5483 & 0.256396 & 0.385126 \\ 0.250000 & & \\ 25.2710 & 0.253101 & 0.381252 \\ 0.250000 & & \\ 25.1440 & 0.251653 & 0.379570 \\ 0.250000 & & \end{array}$

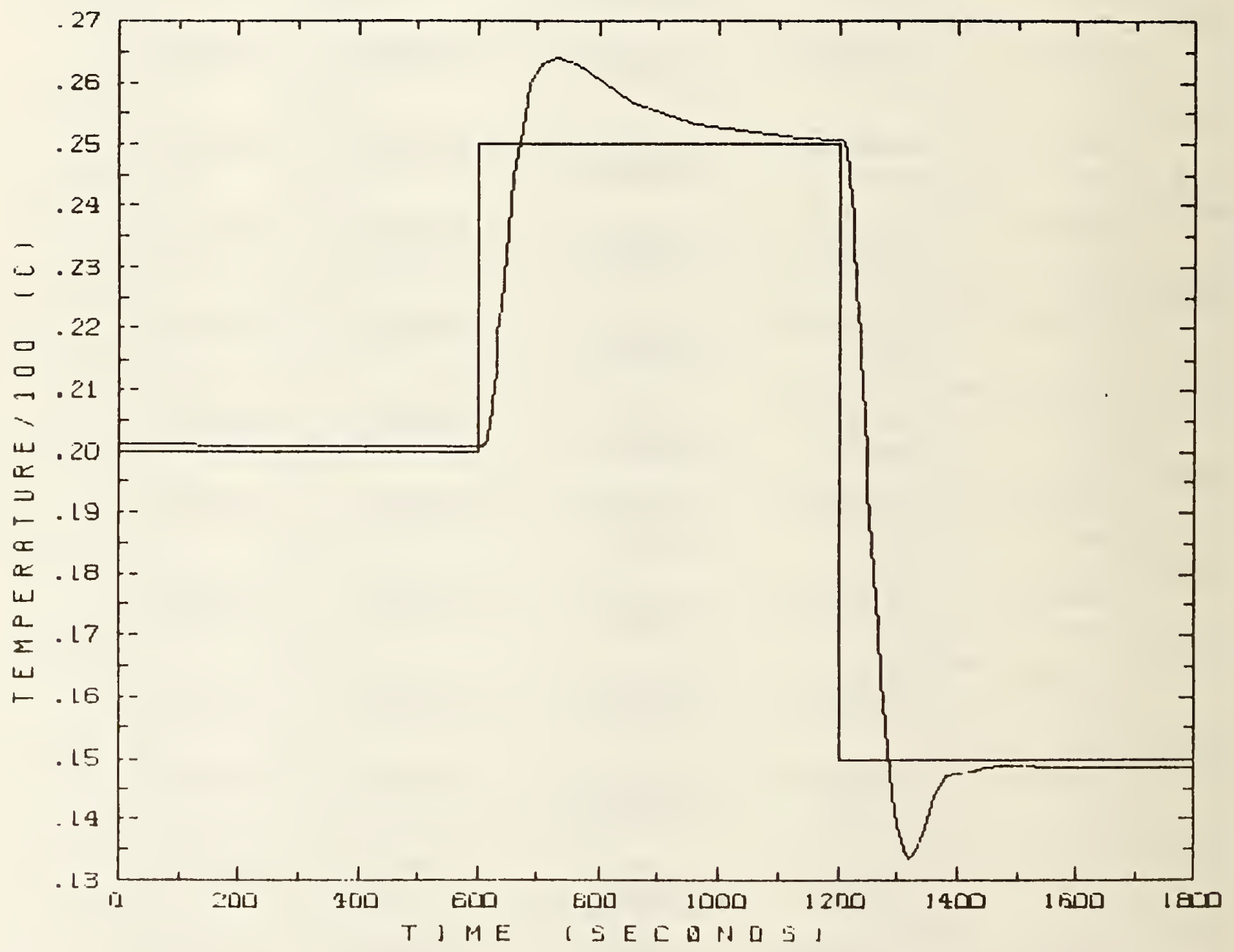

Figure 6.2-3. Controlled variable (air temperature) response to controller set point step changes. 


\subsection{Example 3: Cooling Coil Control Loop With Variable Water Flow Rate}

The previous example represents a control loop in which the flow rate through a heat exchanger remains nearly constant while the inlet water temperature is controlled. The present example models the more typical case where the inlet water temperature remains constant while the flow rate is controlled. The system is shown schematically in Figure 6.3-1. Unlike Example 2, this simulation does not include a pump. Flow is driven by the constant difference between pressures P1 and P6.

The parameters for the cooling coil model were determined for a specific piece of equipment, and the CReate UNit command was used to store these values. Creation of the simulation then required two steps. First, a simulation was created with no heat exchanger. Second, after this simulation was saved, it was edited to insert the previously saved cooling coil unit and to make the necessary input/output connections between the cooling coil and the balance of the simulation.

The TYPE 8 controller model assumes that increasing its control output (C6 in this case) will cause the input controlled variable ( 33 in this case) to increase. To make this assumption valid, it was necessary to place a TYPE 26 reversing relay or control signal inverter between the controller and the control valve. If the TYPE 12 cooling coil were replaced by a heating coil, the TYPE 26 component would have to be eliminated.

Proportional and integral gains for the controller were determined by trial and error, as described in Example 2. The boundary variable file used with this example is shown in Listing 6.3-1. This time the boundary variables are the air inlet temperature and humidity ratio, T4 and HI. The boundary file specifies step changes in both variables simultaneously at times 1800 and 3600 seconds. The second temperature step change is twice the magnitude of the first one. A constant setpoint corresponding to $15 \mathrm{C}$ is maintained throughout the simulation.

Listing 6.3-2 is the simulation summary portion of the output file MODSUM.DAT. This listing contains all the information needed to re-create the simulation description. Listing 6.3-3 is part of the report from the same file, showing the response to the first step change in the boundary file. Figure 6.3-2 is a graph of control variable $\mathrm{C} 3$, the cooling coil outlet air temperature as measured by the temperature sensor and communicated to the controller. Again, the controller proportional and integral gains, $K_{p}$ and $K_{i}$, have been carefully chosen to produce a rapid and essentially oscillation-free response.

In Figure 6.3-3, both $\mathrm{K}_{\mathrm{p}}$ and $\mathrm{K}_{\mathrm{i}}$ have been increased by 50\%, from 6 and 0.08 to 9 and 0.12 . The system response is more rapid, as seen by the relative narrowness of the first peak, but damped oscillations appear in response to the second, larger step change. The greater degree of activity and faster rates of change in this simulation cause HVACSIM to take shorter time steps, increasing both the number of time steps taken and the execution time by about $75 \%$.

Figure 6.3-4 shows the consequences of further increasing the proportional 
gain to 12. This time the second step change input leads to undamped oscillations, with a period of about 92 seconds. The failure of the oscillations to damp out can be attributed to the hysteresis in the control valve, which allows errors to accumulate while the actuator crosses its dead band.

In its effort to track these fast oscillations, HVACSIM decreases the simulation time step dramatically, spending most of its time at the minimum time step and never allowing the step size to exceed six seconds once the oscillations begin. The average time step between simulation times 3600 and 5400 in this example is well under a second. Relative to the simulation of Figure 6.3-2, the number of time steps is increased by a factor of 17.5 and the execution time is increased by a factor of more than 20. Users should be aware that modeling a system which oscillates relatively rapidly can be extremely expensive computationally. There are nearly as many seconds in a day as there are hours in ten years.

\section{COOLING COIL CONTROL LOOP}

Constant temperature, variable flow control

\section{System Schematic}

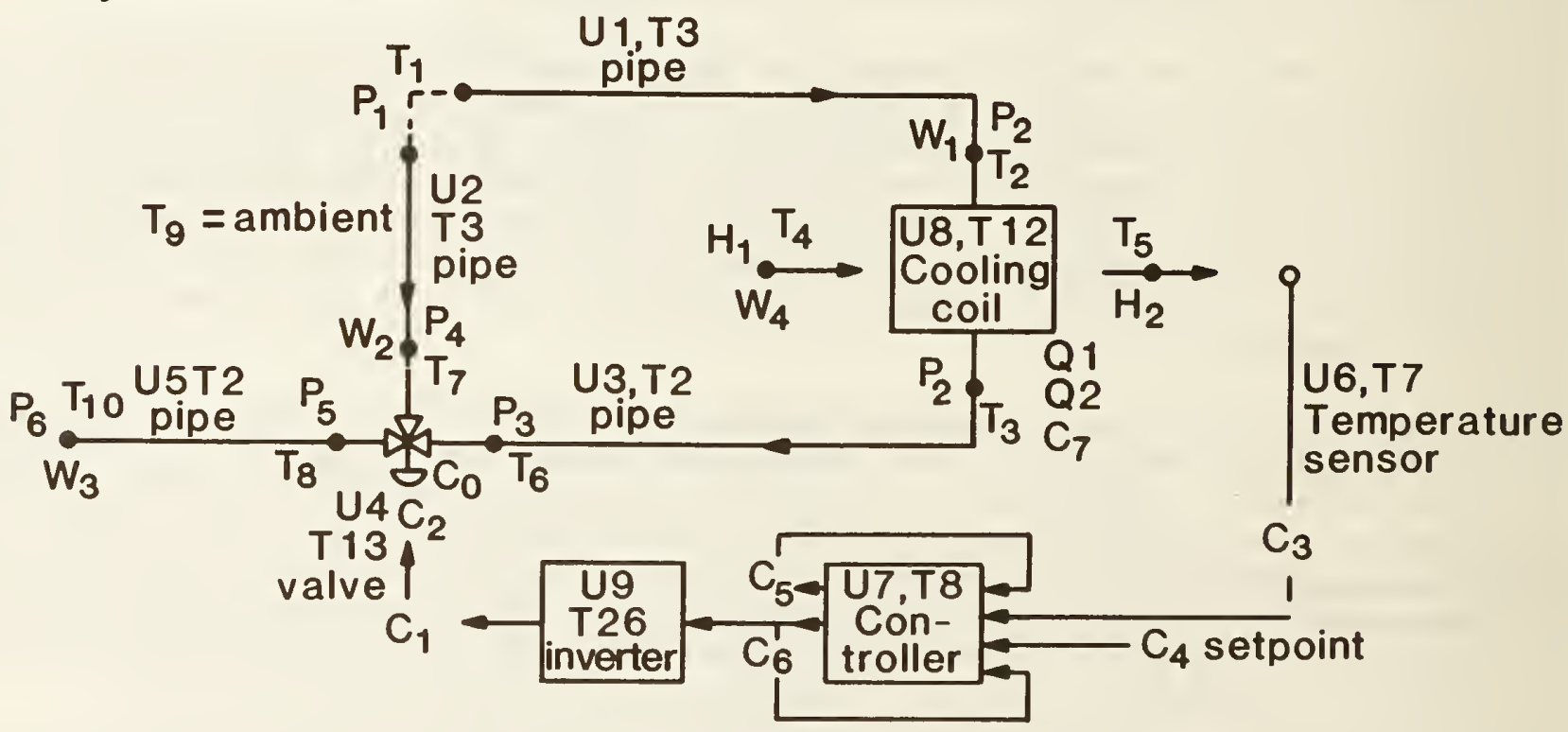

Figure 6.3-1. System schematic for cooling coil control loop 
Listing 6.3-1. Boundary Variable File

$0 ., 25 ., 0.016$

$450 \ldots 25 ., 0.016$

$900 \ldots 25 \ldots 0.016$

$1350 \ldots 25 \ldots 0.016$

$1800 \ldots 25 \ldots, 0.016$

$1800 ., 30 ., 0.022$

$2250 \ldots \quad 30 ., \quad 0.022$

$2700 \ldots \quad 30 ., \quad 0.022$

$3150 \ldots 30 \ldots 0.022$

$3600 \ldots 30 \ldots 0.022$

$3600 \ldots 20 ., 0.010$

$4050 \ldots 20 ., 0.010$

$4500 \ldots 20 ., 0.010$

$4950 ., 20 ., 0.010$

$5400 \ldots 20 ., 0.010$

Listing 6.3-2. Simulation Configuration Sumary from MODSUM.DAT

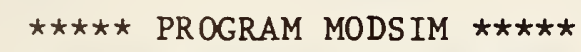

a MODular SIMulation program

COOLING COIL CONTROL LOOP, VARIABLE FLOW, CONSTANT TEMPERATURE

1 SUPERBLOCKS

1 BLOCKS

9 UNITS

31 STATE VARIABLES :

6 PRES 4 FLOW 10 TEMP 7 CTRL 2 POWR 2 AHUM

INITIAL STATE VECTOR:

PRES :

250.000

227.754

212.307

222.467

210.571

$-250.000$

FLOW:

$\begin{array}{llll}1.13930 & 1.49359 & 2.63289 & 2.35000\end{array}$

TEMP :

5.00000

5.01300

16.8008

25.0000

15.0079

16.8056

5.00093

10.1090

25.0000

10.1684

CTRL :

0.424409

0.424409

0.150079

0.150000

0.576066

0.575589

1.00000

POWR :

56.2285

23.8925 
AHUM :

$1.600000 \mathrm{E}-02 \quad 1.062623 \mathrm{E}-02$

2 TIME DEPENDENT BOUNDARY VARIABLES:

TEMP 4 AHUM 1

ERROR TOLERANCES: RTOLX, ATOLX, XTOL, TTIME:

$5.00000 \mathrm{E}-03 \quad 1.00000 \mathrm{E}-06 \quad 5.00000 \mathrm{E}-04 \quad 10.000$

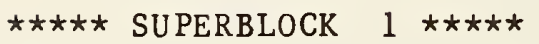

SUPERBLOCK SIMULTANEOUS EQUATION UNFREEZING OPTION, IFZOPT $=0$ SUPERBLOCK INPUT SCAN OPTION, INSOPT $=0$

10 REPORTED VARIABLES:

FLOW 1 CTRL 1 CTRL 2 CTRL 3 CTRL $4 \quad$ CTRL 5 CTRL 6 CTRL 7 AHUM 2 AHUM 1

0 SIMULTANEOUS EQUATIONS; VARIABLES:

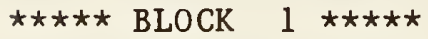

19 SIMULTANEOUS EQUATIONS; VARIABLES:

CTRL 2 CTRL 3 CTRL 5 CTRL 6

$\begin{array}{lllllllll}\text { PRES } 4 & \text { FLOW 1 } & \text { PRES } 3 & \text { TEMP } 7 & \text { FLOW } 2 & \text { TEMP } 5 & \text { AHUM 2 } & \text { PRES } 2 \\ \text { FLOW } 3 & \text { TEMP } 8 & \text { TEMP } 2 & & & & & & \end{array}$

UNIT 1 TYPE 3

5 INPU TS :

$\begin{array}{lllll}\text { PRES } 1 & \text { PRES } 2 & \text { TEMP } 1 & \text { TEMP } 9 & \text { TEMP } 2\end{array}$

2 OUTPUTS :

TEMP 2 FLOW 1

PARAMETERS :

2.9700

$-0.55880$

$3.10000 \mathrm{E}-03 \quad 2.2770$

$2.51000 E-03$

21.360

UNIT 2 TYPE 3

5 IN PUTS:

$\begin{array}{lllll}\text { PRES } 1 & \text { PRES } 4 & \text { TEMP } 1 & \text { TEMP } 9 & \text { TEMP } 7\end{array}$

2 OUTPUTS:

TEMP 7 FLOW 2

PARAMETERS :
0.27600
$-0.55880$
$2.90000 E-04$
0.21160
2.0000
$2.30000 \mathrm{E}-04$
14.800 
UNIT 3 TYPE 2

5 IN PUTS:

FLOW 1 PRES 3 TEMP 3 TEMP 9 TEMP 6

2 OUTPUTS:

TEMP 6 PRES 2

PARAMETERS :
2.7000
$2.81000 \mathrm{E}-03$
2.0700
$2.27800 E-03$
11.900
0.00000
2.0000

UNIT 4 TYPE 13

7 INPUTS :

$\begin{array}{llllllll}\text { FLOW } 1 & \text { FLOW } 2 & \text { PRES } 5 & \text { TEMP } 6 & \text { TEMP } 7 & \text { CTRL } 1 & \text { CTRL } 2\end{array}$

6 OUTPUTS :

CTRL 2 FLOW 3 PRES 3 PRES 4 TEMP 8 NULL 0

PARAMETERS :
0.30000
1.5000
$4.00000 \mathrm{E}-03$
10.000
0.10000
0.00000

UNIT $\quad 5 \quad$ TYPE 2

5 INPUTS :

$\begin{array}{lllll}\text { FLOW } 3 & \text { PRES } 6 & \text { TEMP } 8 & \text { TEMP } 9 & \text { TEMP10 }\end{array}$

2 OUTPUTS:

TEMP10 PRES 5

PARAMETERS :

38.000

4.40000E-02 32.200

$3.55000 \mathrm{E}-02 \quad 66.440$

0.00000

2.0000

UNIT 6 TYPE 7

2 INPUTS:

TEMP 5 CTRL 3

1 OUTPUTS :

CTRL 3

PARAMETERS :

20.000

$0.00000 \quad 100.00$

UNIT $\quad 7 \quad$ TYPE 8

4 IN PU TS :

CTRL 3 CTRL 4 CTRL 5 CTRL 6 
2 OUTPUTS:

CTRL 5 CTRL 6

PARAMETERS :

6.0000

$8.00000 E-02 \quad 2.0000$

UNIT 8 TYPE 12

8 INPUTS :

FLOW 1 TEMP 2 FLOW 4 TEMP 4 AHUM 1 TEMP $3 \quad$ TEMP 5 AHUM 2

6 OUTPUTS :

$\begin{array}{llllll}\text { TEMP } 3 & \text { TEMP } 5 & \text { AHUM } 2 & \text { POWR } 1 & \text { POWR } 2 & \text { CTRL } 7\end{array}$

PARAMETERS :

$\begin{array}{llllc}0.00000 & 6.2381 & 101.71 & 6.2000 & 0.41600 \\ 0.20400 & 0.72260 & 5.5118 & 16.000 & 6.0000 \\ 1.58800 \mathrm{E}-02 & 1.44500 \mathrm{E}-02 & 1.50000 \mathrm{E}-04 & 34.669 & 2.59000 \mathrm{E}-02 \\ 0.50800 & 0.16510 & 0.38600 & & \end{array}$

UNIT 9 TYPE 26

1 INPUTS :

CTRL 6

1 OUTPUTS :

CTRL 1

PARAMETERS :

NONE

TMIN $=\quad 0.100$ TMAX $=900.000$ TSTOP $=5400.000$


Listing 6.3-3. Partial Results of Simulation, from Output File MODSUM.DAT

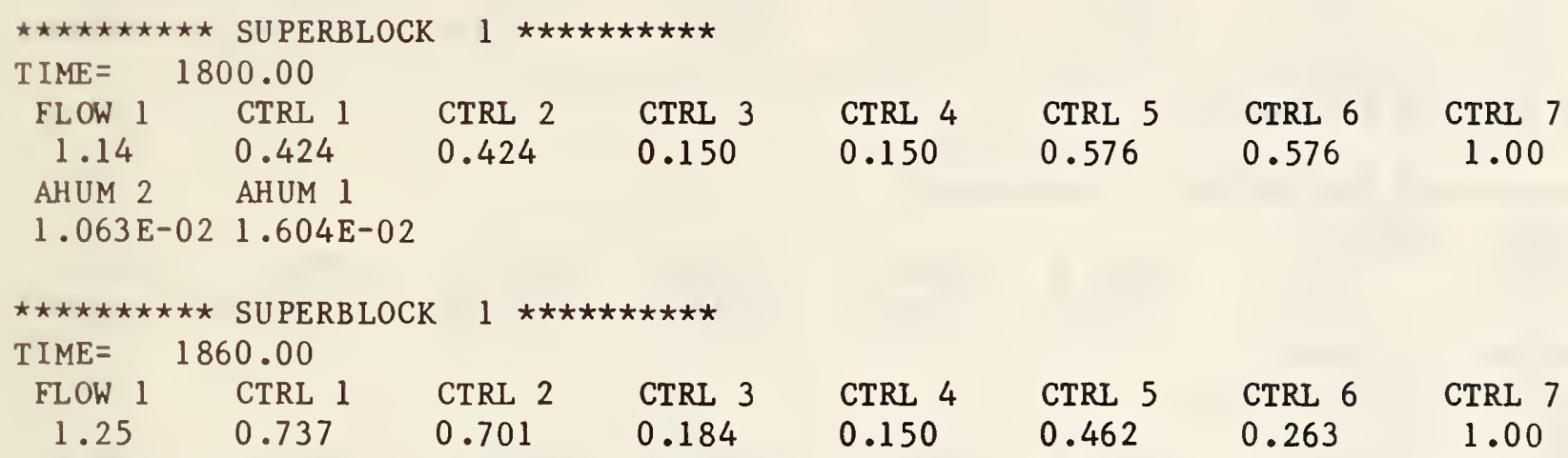




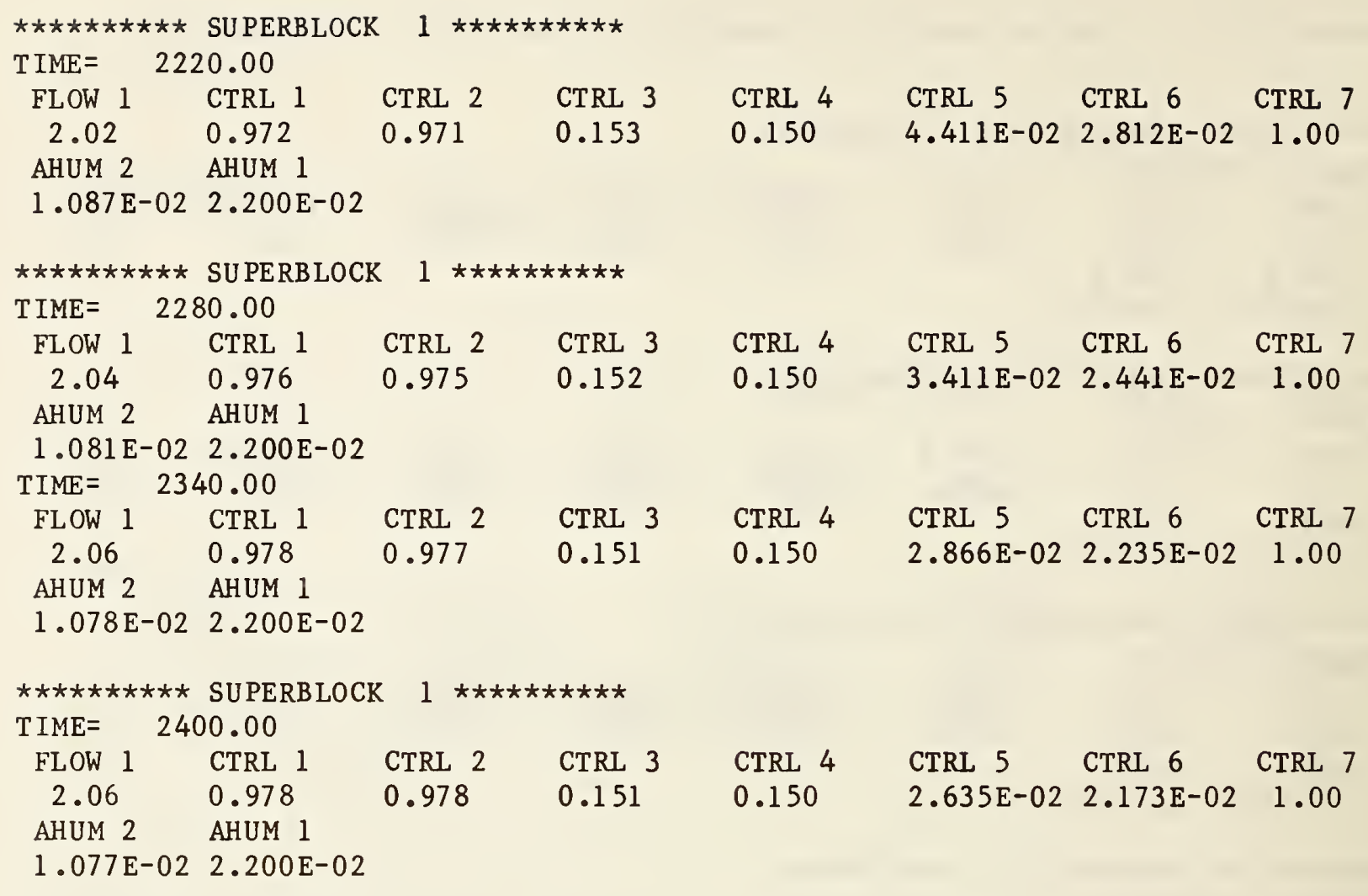




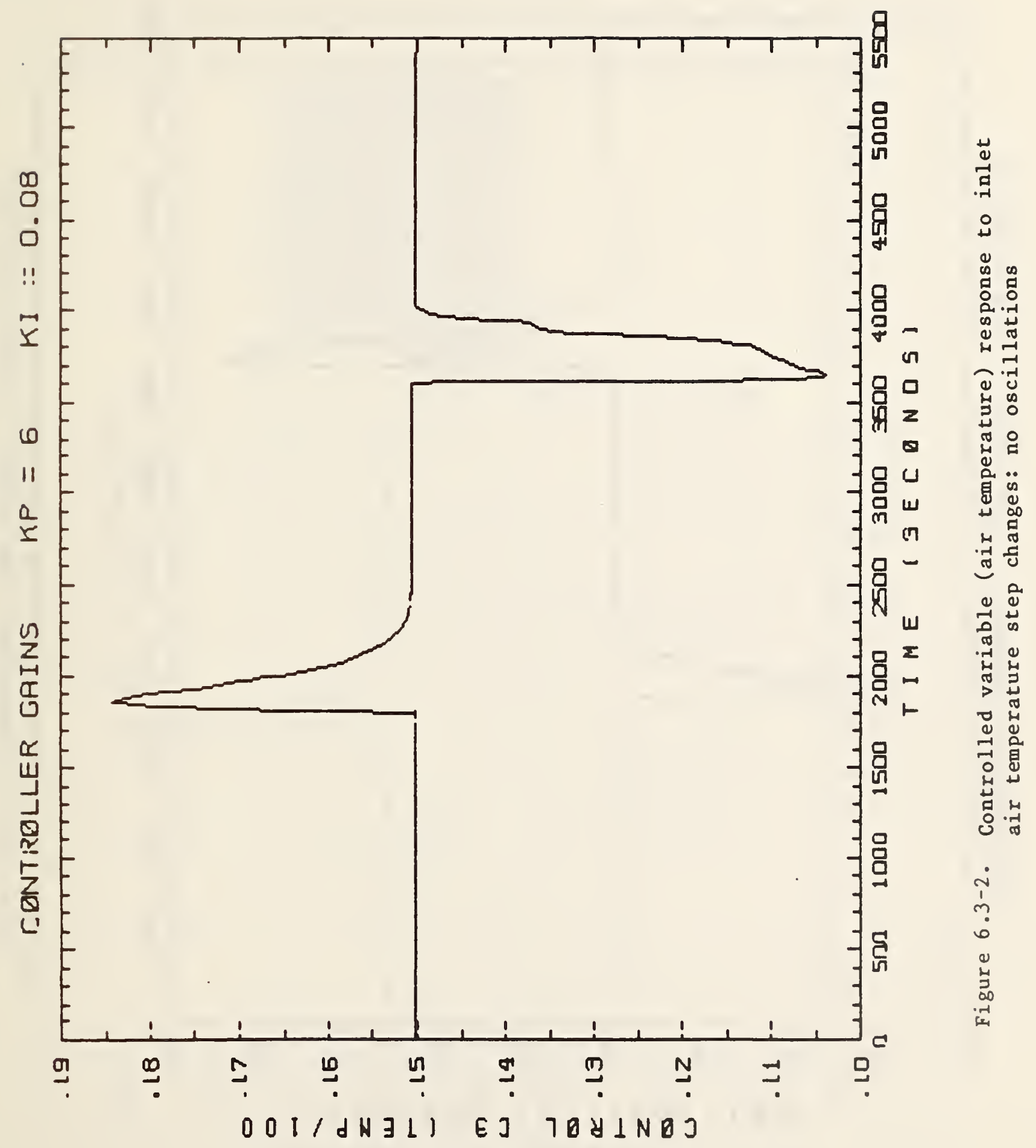




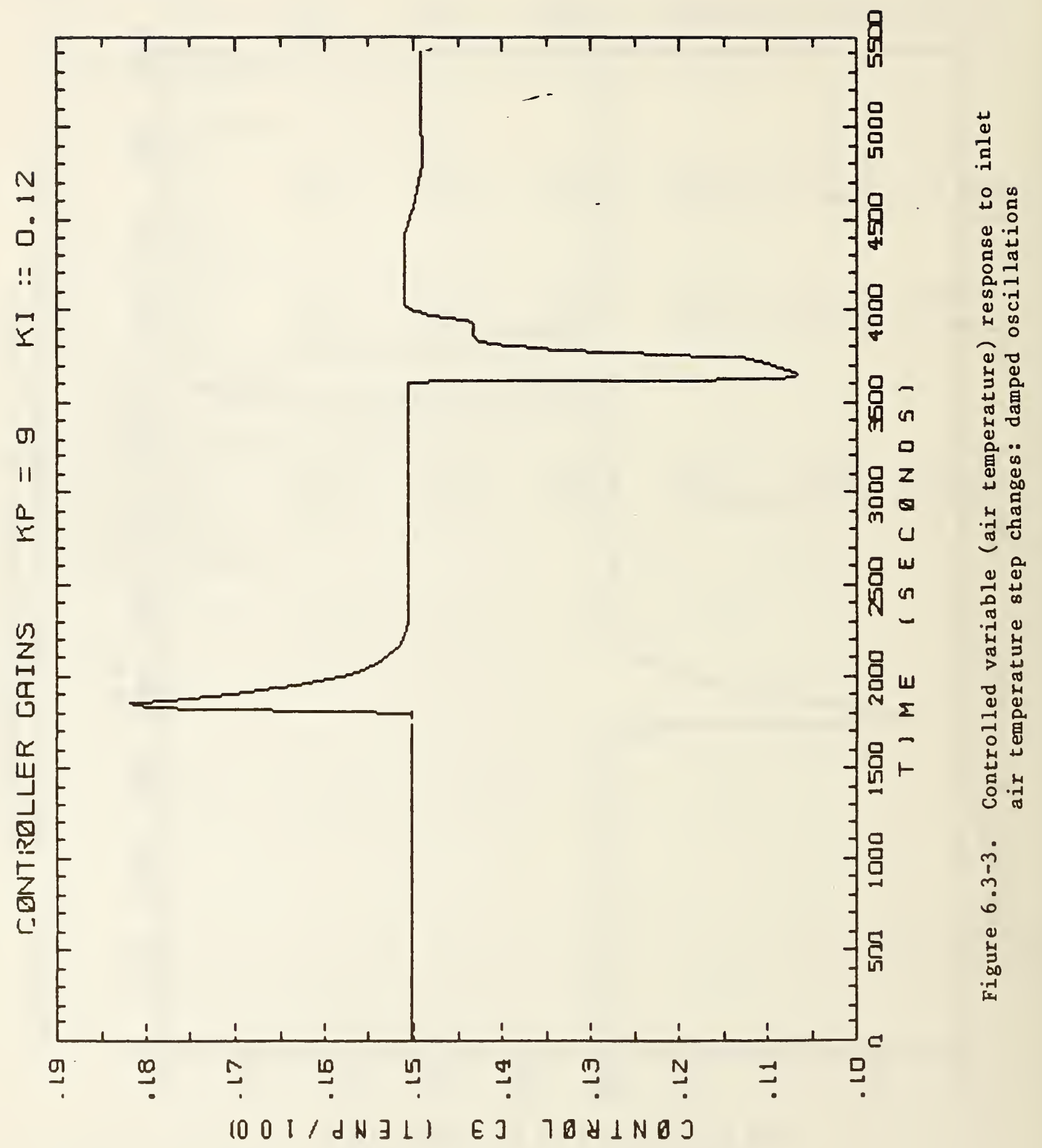




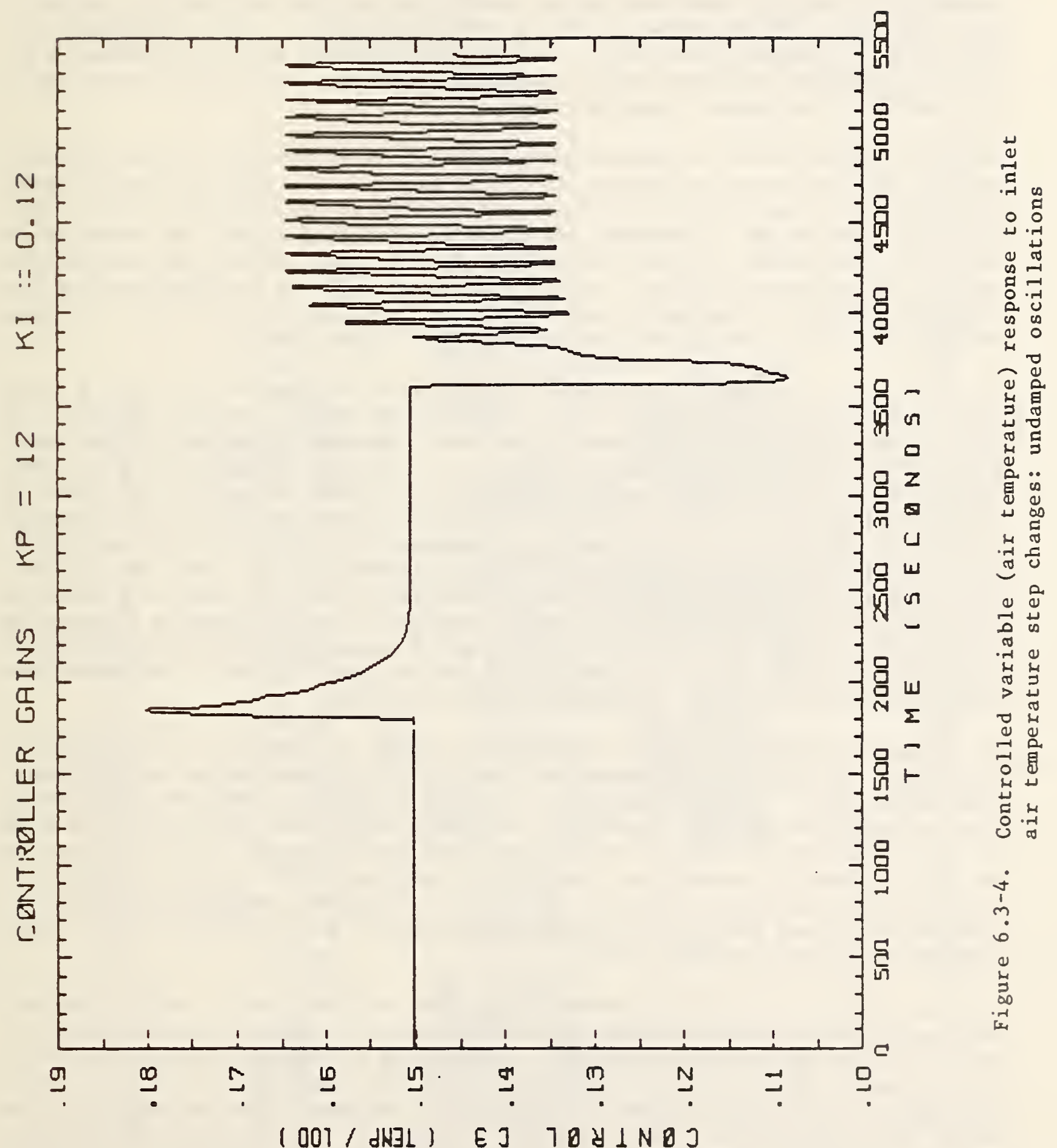




\subsection{Example 4: Air Handling Unit Without Heat Exchangers}

The primary purpose of this example is to check the pressure and flow relationships in an air handler model. Since heat transfer effects will not be considered, dampers have been substituted for heating and cooling coils to represent the flow resistance of the heat exchangers, and a duct model has been used to represent the load. The air handler model will be combined with a heat exchanger and controller subsystem and a more realistic conditioned zone model in subsequent examples.

The air handler model is shown schematically in Figure 6.4-1. The ambient supply air flow rate is labeled $W 2$ in the figure. The Unit 8 , Type 3 Inlet Duct serves to calculate this flow rate and also to make the outdoor air pressure, labeled P6, a boundary condition to the simulation. The presence of the inlet duct model between the Unit 7, Type 5 inlet damper and the outside world is somewhat artificial, but it can be thought of as representing the combined effects of entrance losses and the flow resistance of any ductwork between the Unit 7 damper and the Unit 6, Type 4 Flow Merge.

The return air exhaust flow rate is calculated by the Unit 3, Type 6 Flow Split component. Mass conservation requires that this flow rate be equal to the ambient supply air flow rate, W2, which is already calculated by the inlet duct. The flow rate calculated by Unit 3 is therefore redundant and has been removed from the calculation by assigning it an index of zero. The value of W2 determined by the inlet duct is used as an input to the Unit 4, Type 5 outlet air damper. Alternatively, the exhaust air flow rate calculated by Unit 3 could have been assigned a new index number, such as W4. The difference between W2 and W4, theoretically zero, could then be used as a check on the accuracy of the simulation. Note, however, that the ambient supply and exhaust flow rates calculated by Units 8 and 3 should not both be assigned the same label, since this would make the set of pressure and flow equations overdetermined and could cause convergence problems.

An important feature of this simulation is that it contains no differential equations. Normally, the simulation time step is determined by the differential equations. When only algebraic equations are present, the simulation always takes the maximum time step, with two exceptions: the first time step of the simulation is always the minimum time step, and the last time step is chosen to exactly reach the simulation stopping time. In the absence of differential equations, there is no start-up transient, making initialization unnecessary, and changes in the simulation are driven purely by the time dependent boundary variables.

In this case the only time dependent boundary variable is $\mathrm{Cl}$, which controls the position of three dampers: Units 4, 5, and 7 . When $\mathrm{Cl}$ is zero, Units 4 and 7 are closed and Unit 5 is open, supplying a minimum of fresh outdoor air to the load. When $\mathrm{Cl}$ is one, Units 4 and 7 are open and Unit 5 is closed, supplying a maximum of fresh outdoor air to the load. The boundary file is shown in Listing 6.4-1. Listing 6.4-2 provides the simulation configuration and the simulation results. By interpolation, one can determine the damper position to produce a given percentage of outdoor air in the mixed supply air. For example, the mixed supply air is roughly $25 \%$ ambient air when $\mathrm{C} 1$ is 0.32 . 


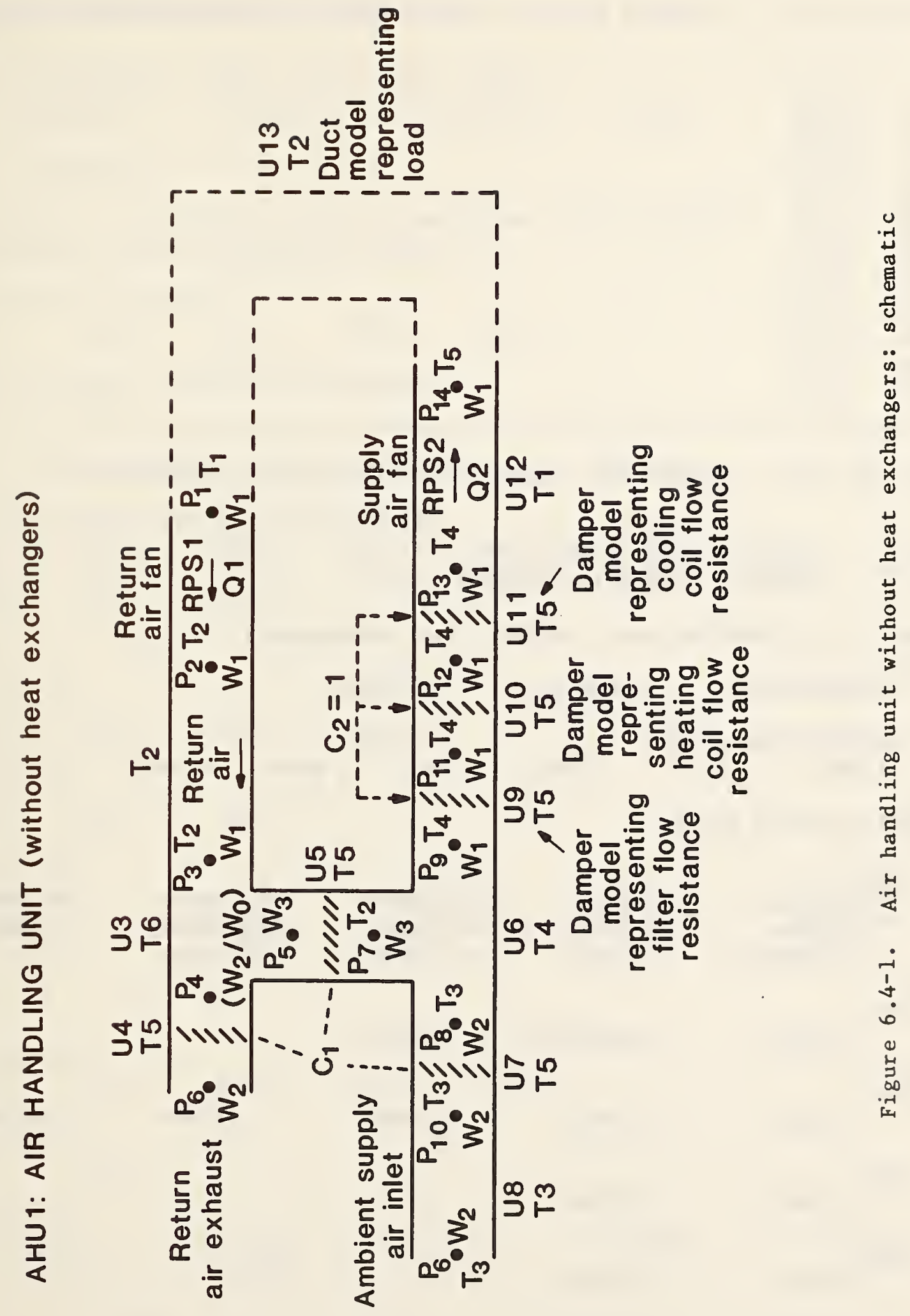


Listing 6.4-1. Boundary Variable File: Values of Time and Control Signal Cl
0.0 .00
1. 0.00
2. 0.05
3. 0.10
4. 0.15
6. 0.25
11. 0.50
16. 0.75
18. 0.85
19. 0.90
20. 0.95
21. 1.00

Listing 6.4-2. MODSUM.DAT: Simulation Configuration and Results

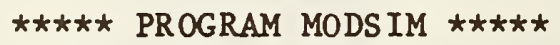

a MODular SIMulation program

AHUl : Air Handling Unit (without heat exchangers)
1 SUPERBLOCKS
1 BLOCKS
13 UNITS

28 STATE VARIABLES:

14 PRES 3 FLOW 5 TEMP 2 CTRL 2 RVPS 2 POWR

INITIAL STATE VECTOR:

PRES :
$-1.75000$
0.250000
0.200000
0.190000
0.160000
0.000000
0.150000
0.150000
$-4.000000$
$-1.000000 \mathrm{E}-06$
$-7.999998 \mathrm{E}-02-0.120000$
$-0.250000$
1.75000

FLOW:
2.00000
$1.000000 \mathrm{E}-02 \quad 1.90000$

TEMP :

25.0000

25.0000

30.0000

26.0000

26.0000

CTRL :

$0.000000 \quad 1.00000$

RV PS :

$60.0000 \quad 60.0000$

POWR :
0.000000
0.000000 
1 TIME DEPENDENT BOUNDARY VARIABLES:

CTRL 1

ERROR TOLERANCES: RTOLX, ATOLX, XTOL, TTIME:

$$
\begin{array}{llll}
1.00000 \mathrm{E}-04 & 1.00000 \mathrm{E}-05 \quad 2.00000 \mathrm{E}-04 & 1.0000
\end{array}
$$

$\star \star \star \star \star$ SUPERBLOCK $1 \star \star \star \star \star \star$

SUPERBLOCK SIMULTANEOUS EQUATION UNFREEZING OPTION, IFZOPT $=0$

SUPERBLOCK INPUT SCAN OPTION, INSOPT $=0$

5 REPORTED VARIABLES :

PRES14 FLOW 1 FLOW 2 TEMP 4 CTRL 1

0 SIMULTANEOUS EQUATIONS; VARIABLES:

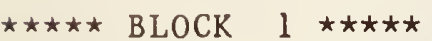

19 SIMULTANEOUS EQUAT IONS; VARIABLES :

$\begin{array}{llllllllll}\text { PRES } 2 & \text { FLOW } 1 & \text { TEMP } 2 & \text { PRES } 3 & \text { PRES } 4 & \text { PRES } 5 & \text { FLOW } 2 & \text { FLOW } 3\end{array}$

$\begin{array}{llllllll}\text { PRES } 7 & \text { PRES } 9 & \text { PRES } 8 & \text { PRES10 } & \text { PRES11 } & \text { PRES12 } & \text { PRES13 } & \text { TEMP } 4\end{array}$

PRES14 PRES 1 TEMP 5

UNIT 1 TYPE 1

4 IN PUTS :

FLOW 1 PRES 2 RVPS 1 TEMP 1

3 OUTPUTS :

PRES 1 TEMP 2 POWR 1

PARAMETERS :

3.6400

0.80100

$-0.19000$

$-4.45000 \mathrm{E}-03$

0.00000

0.00000

0.56400

$-8.62000 \mathrm{E}-02$

0.00000

0.00000

0.33650

1.0000

UNIT 2 TYPE 2

5 IN PUTS :

FLOW 1 PRES 3 TEMP 2 TEMP 2 TEMP 2

2 OUTPUTS:

NULL 0 PRES 2

PARAMETERS :

0.00000

0.00000

$$
0.00000
$$

0.00000

0.00000

UNIT $3 \quad$ TYPE 6

3 INPUTS : 
FLOW 1 PRES 4 PRES 5

3 OUTPUTS:

NULL 0 FLOW 3 PRES 3

\section{PARAMETERS :}

$1.10000 \mathrm{E}-02$

UNIT 4 TYPE 5

3 INPUTS:

FLOW 2 PRES 6 CTRL 1

1 OUTPUTS :

PRES 4

PARAMETERS :

$\begin{array}{llll}1.00000 \mathrm{E}-03 & 1.20000 \mathrm{E}-02 & 0.87000 & 0.00000\end{array}$

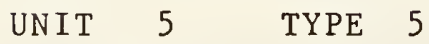

3 IN PUTS :

FLOW 3 PRES 7 C.TRL 1

1 OUTPUTS :

PRES 5

PARAMETERS :

$\begin{array}{llll}1.00000 \mathrm{E}-03 & 1.20000 \mathrm{E}-02 & 0.87000 & 1.0000\end{array}$

UNIT $\quad 6 \quad$ TYPE $\quad 4$

5 IN PU TS :

FLOW 3 FLOW 2 PRES 9 TEMP 2 TEMP 3

4 OUTPUTS :

FLOW 1 PRES 7 PRES 8 TEMP 4

PARAMETERS :

$1.10000 \mathrm{E}-02$

UNIT 7 TYPE 5

3 INPUTS :

FLOW 2 PRES 8 CTRL 1

1 OUTPUTS :

PRESI 0

PARAMETERS :
$1.00000 \mathrm{E}-03$
$1.20000 \mathrm{E}-02$
0.87000
0.00000 
UNIT 8 TYPE 3

5 IN PUTS :

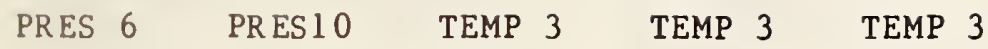

2 OUTPUTS:

NULL 0 FLOW 2

PARAMETERS :

0.00000

0.00000

$$
0.00000
$$

0.00000

0.00000

$1.00000 E-03$

UNIT 9 TYPE 5

3 INPUTS :

FLOW 1 PRESI1 CTRL 2

1 OUTPUTS :

PRES 9

PARAMETERS :

$$
\begin{array}{llll}
4.00000 E-02 & 4.00000 E-05 & 1.0000 & 0.00000
\end{array}
$$

UNIT $10 \quad$ TYPE 5

3 IN PUTS :

FLOW 1 PRES12 CTRL 2

1 OUTPUTS :

PRES 11

PARAMETERS :

$\begin{array}{llll}1.20000 E-02 & 1.20000 E-05 & 1.0000 & 0.00000\end{array}$

UNIT 11 TYPE 5

3 IN PUTS:

FLOW 1 PRESI3 CTRL 2

1 OUTPUTS:

PRES 12

PARAMETERS :

$\begin{array}{llll}4.00000 \mathrm{E}-02 & 4.00000 \mathrm{E}-05 & 1.0000 & 0.00000\end{array}$

UNIT 12 TYPE 1

4 INPUTS :

FLOW 1 PRESI4 RVPS 2 TEMP 4

3 OUTPUTS :

PRES13 TEMP 5 POWR 2 
PARAMETERS :

$\begin{array}{lllll}3.6400 & 0.80100 & -0.19000 & -4.45000 \mathrm{E}-03 & 0.00000 \\ 0.00000 & 0.56400 & -8.62000 \mathrm{E}-02 & 0.00000 & 0.00000 \\ 0.33650 & 1.0000 & & & \end{array}$

UNIT 13 TYPE 2

5 IN PUTS :

FLOW 1 PRES 1 TEMP 5 TEMP 5 TEMP 5

2 OUTPUTS :

NULL 0 PRES14

PARAMETERS :
0.00000
0.00000
0.00000
500.00
0.87500
0.00000
1.0000

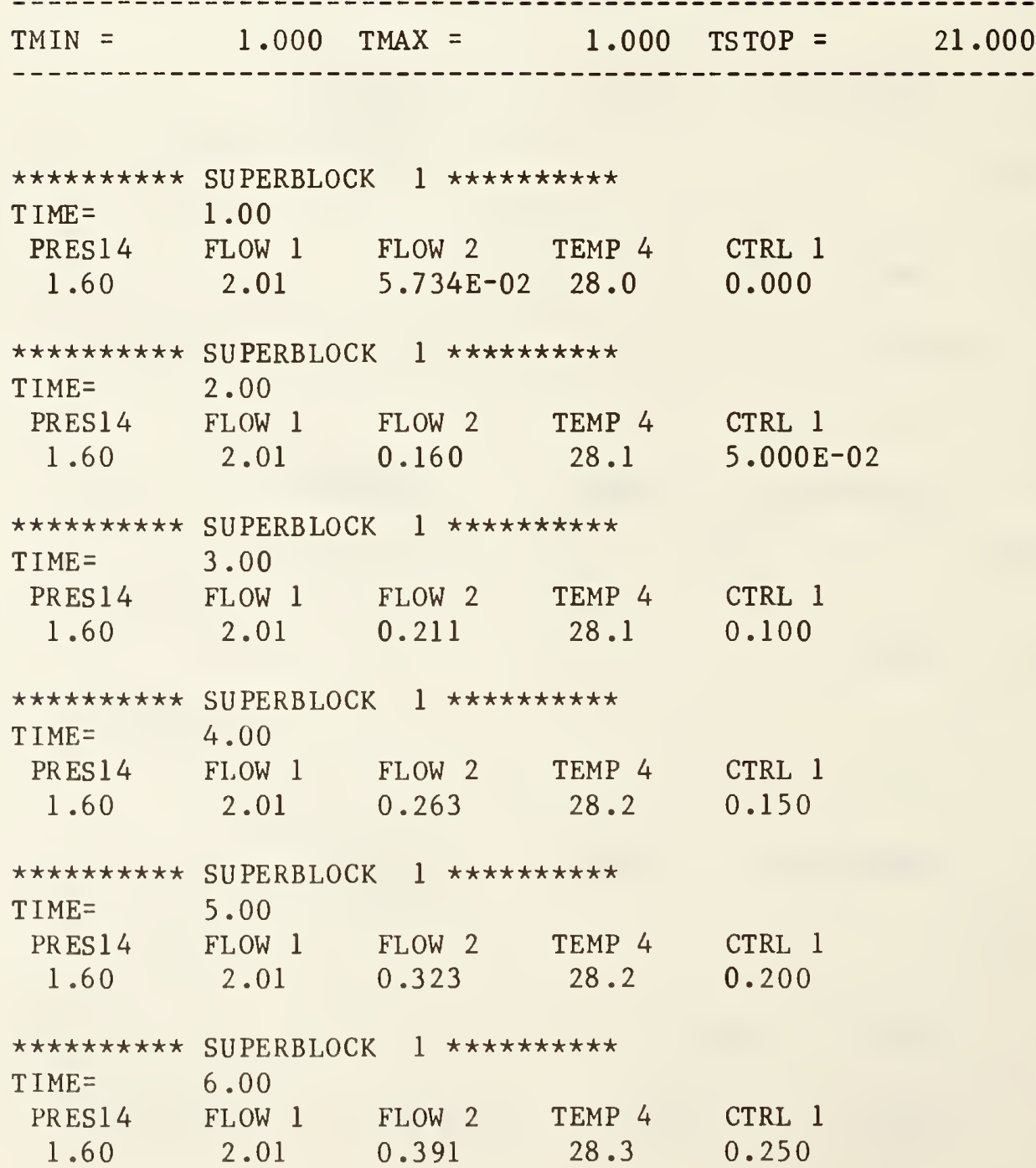




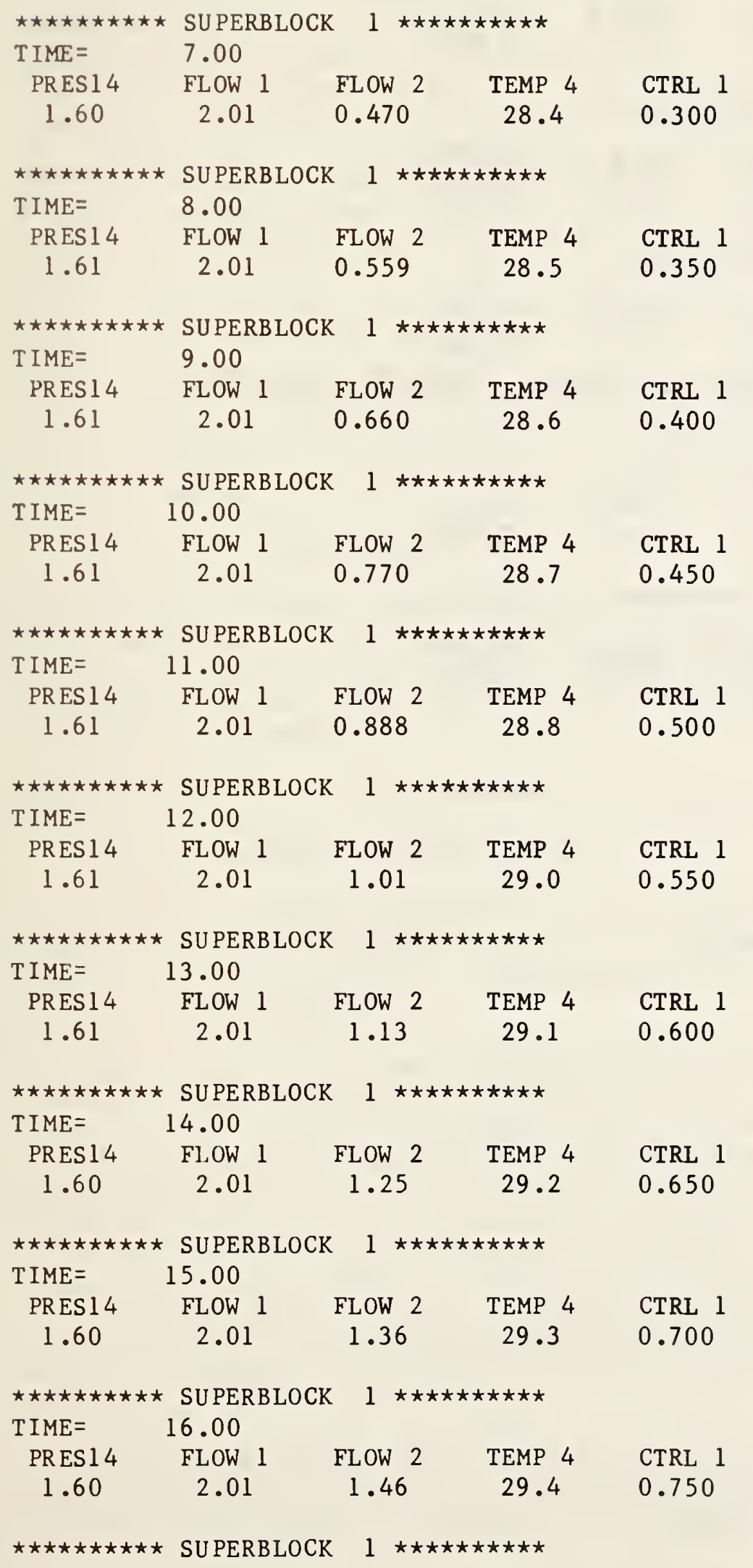




\begin{tabular}{|c|c|c|c|c|c|}
\hline TIME $=$ & 17.00 & & & & \\
\hline PRES 14 & FLOW 1 & FLOW 2 & TEMP 4 & CTRL 1 & \\
\hline 1.60 & 2.01 & 1.55 & 29.5 & 0.800 & \\
\hline 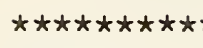 & * SUPERBI & 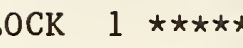 & 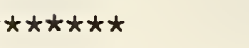 & & \\
\hline $\mathrm{T} I M E=$ & 18.00 & & & & \\
\hline PRES 14 & FLOW 1 & FLOW 2 & TEMP 4 & CTRL 1 & \\
\hline 1.60 & 2.01 & 1.63 & 29.6 & 0.850 & \\
\hline$\star \star \star \star \star \star \star \star \star \star \star ~$ & * SUPERBI & OCK $1 * \star \star \star \star \star x$ & 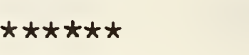 & & \\
\hline TIME $=$ & 19.00 & & & & \\
\hline PRES 14 & FLOW 1 & FLOW 2 & TEMP 4 & CTRL 1 & \\
\hline 1.60 & 2.01 & 1.70 & 29.7 & 0.900 & \\
\hline$\star \star \star \star \star \star E N D$ & OF FILE & ENCOUNTERED & ON BOUNDARY & Y CONDITION FILE AT TIME & 20.00 \\
\hline 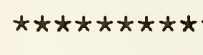 & * SUPERBI & 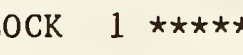 & 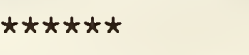 & & \\
\hline TIME $=$ & 20.00 & & & & \\
\hline PRES 14 & FLOW 1 & FLOW 2 & TEMP 4 & CTRL 1 & \\
\hline 1.60 & 2.01 & 1.77 & 29.8 & 0.950 & \\
\hline 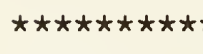 & * SUPERBI & 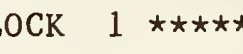 & 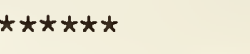 & & \\
\hline TIME= & 21.00 & & & & \\
\hline PRES14 & FLOW 1 & FLOW 2 & TEMP 4 & CTRL 1 & \\
\hline 1.60 & 2.01 & 1.92 & 29.9 & 1.00 & \\
\hline
\end{tabular}




\subsection{Example 5: Air Handler With Flow-Controlled Cooling Coil}

In this example, the cooling coil control loop of Example 3 and the air handling unit of Example 4 are combined into a single simulation consisting of two blocks, as shown in Figure 6.5-1. The two blocks in this figure are coupled through their common use of three state variables: T4, T5, and W4, the air temperatures entering and leaving the cooling coil and the air flow rate through the coil. The damper model representing a pressure drop across the coil has been retained, since the cooling coil model does not calculate a pressure drop itself. Thus Unit 8 in Block 1 represents the thermal effects of the coil, and Unit 20 in Block 2 represents the flow resistance of the coil. The procedure for combining blocks from different simulations, using the editing features of HVACGEN, is described below.

First, the SAve command from the EDit menu is used to extract a block (in the present example, the only block) from one of the existing simulation work files and write it to a block file. In this case the following commands are entered, either sequentially or on a single line:

\section{EDIT SIMULATION EXAMPLE4 SAVE}

In response to prompts from HVACGEN, Block 1 is identified as the block to be saved and the file name EXAMPLE4 is given approval. The file EXAMPLE4.SIM remains unaltered, and a new file named EXAMPLE4.BLK is created. HVACGEN returns to the EDit menu, and control is returned to the main menu by entering the continue command.

Next, the following command sequence is entered:

\section{EDIT SIMULATION EXAMPLE3 INSERT}

In response to prompts, HVACGEN is told to insert a block into superblock 1 of EXAMPLE3, to read the block from the file EXAMPLE4, to automatically renumber the state variable indices in the inserted block, and to save the results in a new file named EXAMPLE5.

At this point a file named EXAMPLE5.SIM has been created and contains two blocks which are completely independent of each other, having no variables in common. For example, since Block 1 contains temperatures Tl through TlO, an offset of 10 has been added to all temperatures in Block 2, which are now numbered Tll through Tl5. Similarly, the three flow rates in Block 2 are now numbered $W 5, W 6$, and $W 7$, since Block 1 contains four flow rates. Before the simulation can be used, the Units in Block 2 must be edited one by one, changing variable indices as necessary to make the simulation description correspond to Figure 6.5-1. (A "substitute" command, which would (for example) automatically find all occurrences of W5 and change them all to W4, could be very helpful here, but had not been implemented at the time this was written.)

Actually, more changes were made here than is strictly necessary. For example, changing flow W5 to W4 was necessary, but changing W6 to W5 and W7 to W6 was not. If $W 6$ and $W 7$ had been left unchanged, $W 5$ would be an unused variable 
name, increasing the size of the state vector slightly but doing no real harm. Alternatively, the gap could have been filled by changing W7 to W5, leaving W6 alone.

A block file does not contain initial values of variables. When a block is inserted, the new state variables it contains are all assigned initial values of zero by HVACGEN. This is true whether the block is read from a file or created interactively, and it also applies to the insertion of units within a block. Whenever a simulation is edited and new variable names are generated, the EDit VAriables command should be used to provide initial values for the new variables. Again, in this example more changes were made than is strictly necessary: many of the initial values of variables in Block 1 were also modified.

The simulation title, boundary variables, reported variables, reporting interval, and error tolerances are still the same as in EXAMPLE3, from which EXAMPLE5 is derived. It is usually desirable to edit these categories of information as well. A summary of the final simulation configuration and a sample of the simulation output are contained in Listing 6.5-1. Notice that all time dependent boundary conditions have been removed from this example. The system works its way from the initial conditions to steady state, and will stay there indefinitely. Figure 6.5-2 is a graph of the controlled temperature leaving the cocling coil, T5, and the temperature leaving the "1oad," Tll, during this simulation startup transient. 
AIR HANDLING UNIT WITH COOLING COIL

Block 1

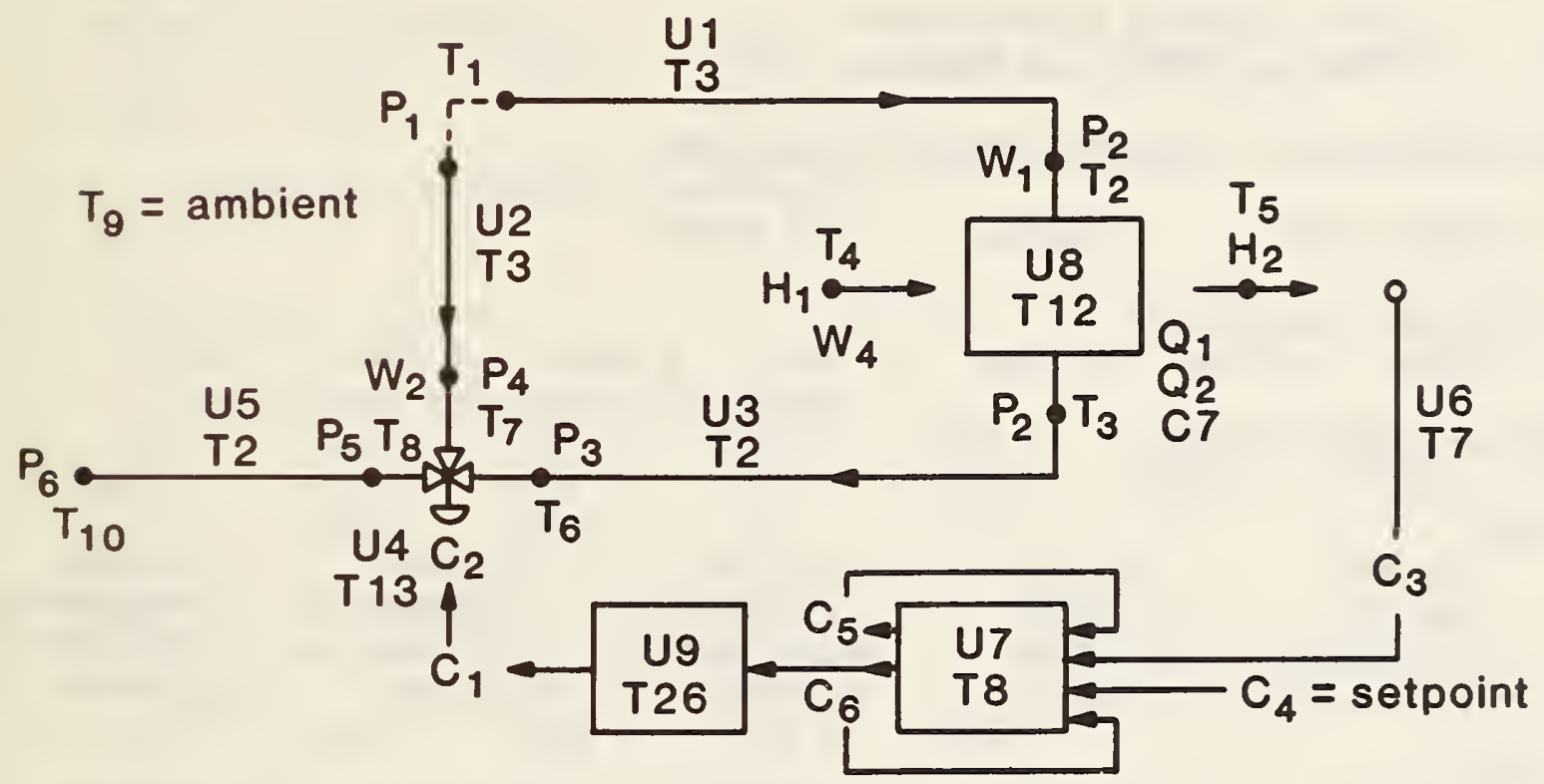

Block 2

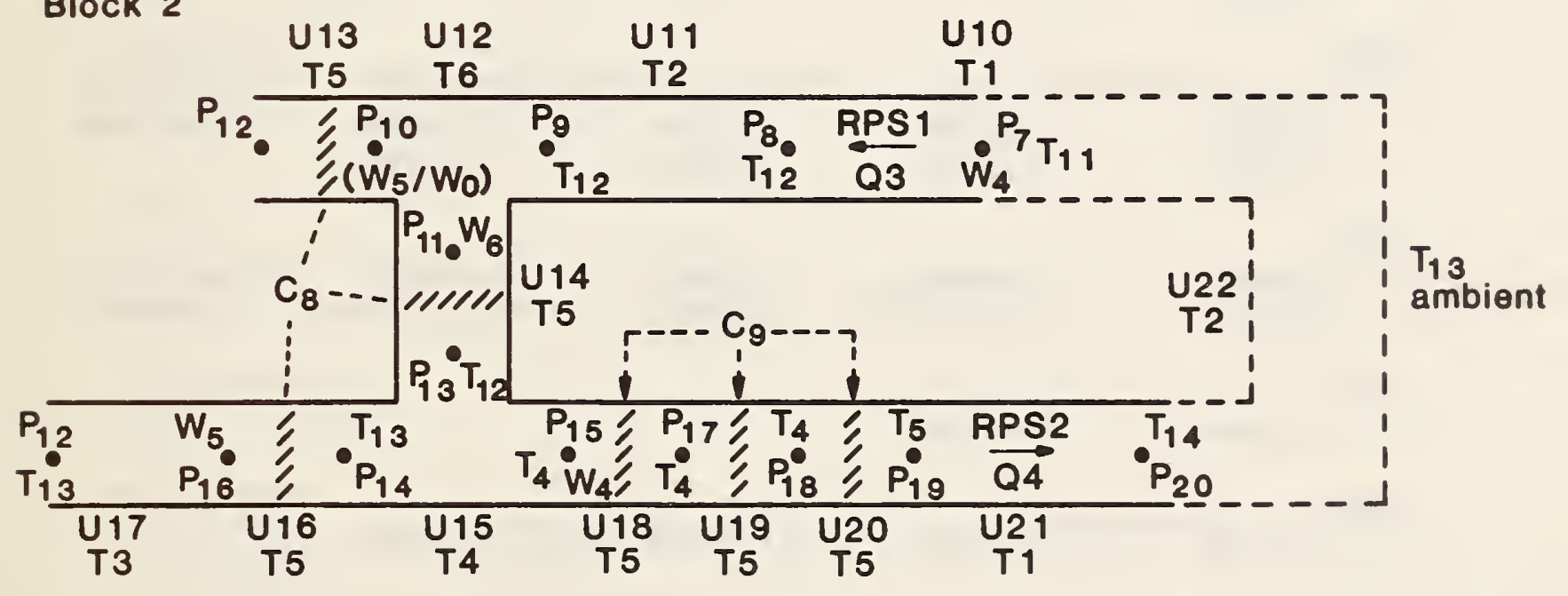

Figure 6.5-1. Air handling unit with cooling coil control loop 
Listing 6.5-1. MODSUM.DAT: Simulation Summary and Output

$\star \star \star \star \star$ PROGRAM MODSIM $\star \star \star \star \star \star$
a MODular SIMulation program

AIR HANDLER WITH FLOW-CONTROLLED COOLING COIL

1 SUPERBLOCKS 2 BLOCKS 22 UNITS

58 STATE VARIABLES:

20 PRES 6 FLOW 14 TEMP 10 CTRL 2 RVPS 4 POWR 2 AHUM

INITIAL STATE VECTOR:

PRES :

$\begin{array}{crccc}250.000 & 227.600 & 212.100 & 222.600 & 210.400 \\ -250.000 & -1.94000 & 7.975000 \mathrm{E}-02 & 3.926000 \mathrm{E}-02 & 1.560000 \mathrm{E}-02 \\ 4.492998 \mathrm{E}-03 & 0.000000 & -4.747000 \mathrm{E}-03 & -1.585000 \mathrm{E}-02 & -3.952000 \mathrm{E}-02 \\ -2.544001 \mathrm{E}-04 & -0.201500 & -0.250000 & -0.412000 & 1.60500\end{array}$

FLOW :

$\begin{array}{lllll}1.14200 & 1.49100 & 2.63300 & 2.00000 & 0.500000 \\ 1.50000 & & & \end{array}$

TEMP :

5.00000

5.01300

16.7900

32.0000

20.0000

16.8000

5.00000

10.1200

25.0000

10.1800

27.0000

28.0000

35.0000

22.0000

CTRL :

0.428000

0.428000
1.00000

0.220000

0.320000

0.180000

1.00000

0.572000

0.572000

30.0000

30.0000

POWR :

56.3000

24.0000

9.20000

9.20000

AHUM :

$1.500000 \mathrm{E}-02 \quad 4.999999 \mathrm{E}-03$

0 TIME DEPENDENT BOUNDARY VARIABLES:

ERROR TOLERANCES: RTOLX, ATOLX, XTOL, TTIME:

$5.00000 \mathrm{E}-03 \quad 1.00000 \mathrm{E}-06 \quad 5.00000 \mathrm{E}-04 \quad 10.000$

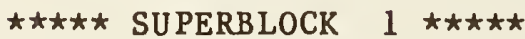


SUPERBLOCR SIMUTIANEOOS EQUATION UNFREEZING OPTION, IFZOPT $=2$

SUPERBLOCR INPOT SCAN OPTION, INSOPT = 0

10 REPORTED VARIABLES:

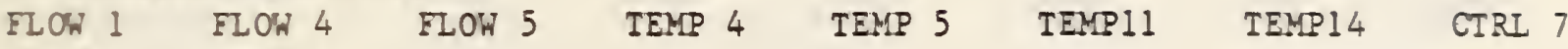
CTRLIO CTRL 1

1 SIMULTANEOOS BQUATIONS; VARIABLES:

TEMP 4

$\star \star \star \star \star$ BLOCR 1

19 SIMUI TANEOUS EQUAIIONS: VARIABLES:

CTRL 2 CTRL 3 CTRI 5 CTRI 6

PRES 4 FLOW 1 PRES 3 TEMP 7 FLOW 2 TEMP 6 PRES 5 CTRL 1

FIOW 3 TEMP 8 TEMP 2

UNIT 1 TYPE 3

5 INPUTS :

PRES 1 PRES 2 TEMP 1 TEMP 9 TEMP 2

2 OUTPOIS:

TEMP 2 FLOW 1

PARAMETERS :

$\begin{array}{rcccc}2.9700 & 3.10000 E-03 & 2.2770 & 2.51000 E-03 & 21.360 \\ -0.55880 & 2.0000 & & \end{array}$

UNIT 2 TYPE 3

5 INPUTS :

$\begin{array}{lllll}\text { PRES } 1 & \text { PRES } 4 & \text { TEMP } 1 & \text { TEMP } 9 & \text { TEMP } 7\end{array}$

2 OUIPUTS:

TEMP 7 FLOW 2

PARAMETERS :

0.27600

$-0.55880$

$$
\begin{aligned}
& 2.90000 E-04 \quad 0.21160 \\
& 2.0000
\end{aligned}
$$

$2.30000 E-04$

14.800

UNIT 3 TYPE 2

5 INPUTS:

FLON 1 PRES 3 TEMP 3 TEMP 9 TEMP 6

2 OUTPUTS:

TEMP 6 PRES 2

PARAMETERS :

2.7000

0.00000

$2.81000 E-03$

2.0700

$2.27800 E-03$

11.900 
UNIT 4 TYPE 13

7 INPUTS:

FLOW 1 FLOW 2 PRES 5 TEMP 6 TEMP $7 \quad$ CTRL 1 CTRL 2

6 OUTPUTS:

$\begin{array}{llllll}\text { CTRL } 2 & \text { FLOW } 3 & \text { PRES } 3 & \text { PRES } 4 & \text { TEMP } 8 & \text { CTRL10 }\end{array}$

PARAMETERS :

0.30000

$1.5000 \quad 4.00000 \mathrm{E}-03 \quad 10.000 \quad 0.10000$

0.00000

UNIT 5 TYPE 2

5 INPUTS:

FLOW 3 PRES 6 TEMP 8 TEMP 9 TEMP10

2 OUTPUTS:

TEMP10 PRES 5

PARAMETERS :

38.000

0.00000

4.40000E-02

2.0000

32.200

$3.55000 \mathrm{E}-02$

66.440

$\begin{array}{llll}\text { UNIT } & 6 & \text { TYPE } & 7\end{array}$

2 INPUTS:

TEMP 5 CTRL 3

1 OUTPUTS :

CTRL 3

PARAME TERS :

20.000

0.00000

100.00

$\begin{array}{llll}\text { UNIT } & 7 & \text { TYPE } & 8\end{array}$

4 INPUTS :

CTRL 3 CTRL 4 CTRL 5 CTRL 6

2 OUTPUTS:

CTRL 5 CTRL 6

PARAMETERS :

$6.0000 \quad 8.00000 \mathrm{E}-02 \quad 2.0000$

$\begin{array}{llll}\text { UNIT } & 8 & \text { TYPE } & 12\end{array}$

8 INPUTS:

FLOW 1 TEMP 2 FLOW 4 TEMP 4 AHUM 1 TEMP 3 TEMP 5 AHUM 2 
6 OUTPUTS :

$\begin{array}{llllll}\text { TEMP } 3 & \text { TEMP } 5 & \text { AHUM } 2 & \text { POWR } 1 & \text { POWR } 2 & \text { CTRL } 7\end{array}$

PARAMETERS :
0.00000
6.2381
101.71
0.20400
0.72260
5.5118
6.2000
16.000
0.41600
6.0000
$1.58800 \mathrm{E}-02$
$1.44500 \mathrm{E}-02$
$1.50000 \mathrm{E}-04$
34.669
$2.59000 \mathrm{E}-02$
0.50800
0.16510
0.38600

UNIT 9 TYPE 26

1 INPUTS :

CTRL 6

1 OUTPUTS:

CTRL 1

PARAME TERS :

NONE

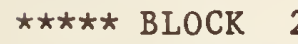

19 SIMULTANEOUS EQUATIONS; VARIABLES:

$\begin{array}{llllllll}\text { PRES } 8 & \text { FLOW 4 } & \text { TEMP11 } & \text { TEMP12 } & \text { PRES 9 } & \text { PRES10 } & \text { PRES11 } & \text { FL OW } 5 \\ \text { FLOW 6 } & \text { PRES13 } & \text { PRES15 } & \text { PRES14 } & \text { PRES16 } & \text { PRES17 } & \text { PRES18 } & \text { PRES19 }\end{array}$

PRES 20 PRES 7 TEMP14

UNIT 10 TYPE 1

4 IN PUTS :

FLOW 4 PRES 8 RVPS 1 TEMP11

3 OUTPUTS :

PRES 7 TEMP12 POWR 3

PARAMETERS :

NONE

UNIT 11 TYPE 2

5 INPUTS:

$\begin{array}{lllll}\text { FLOW } 4 & \text { PRES } 9 & \text { TEMP 12 } & \text { TEMP12 } & \text { TEMP12 }\end{array}$

2 OUTPUTS:

NULL 0 PRES 8

PARAMETERS :
0.00000
0.00000
0.00000
0.00000
0.00000

$\begin{array}{llll}\text { UNIT } & 12 & \text { TYPE } & 6\end{array}$ 
3 INPUTS:

FLOW 4 PRES10 PRES11

3 OUTPUTS:

NULL 0 FLOW 6 PRES 9

PARAMETERS :

1.10000E-02

UNIT 13 TYPE 5

3 INPUTS:

FLOW 5 PRES12 CTRL 8

1 OUTPUTS:

PRES10

PARAMETERS:

$\begin{array}{llll}1.00000 \mathrm{E}-03 & 1.20000 \mathrm{E}-02 & 0.87000 & 0.00000\end{array}$

UNIT 14 TYPE 5

3 INPUTS:

FLOW 6 PRES13 CTRL 8

1 OUTPUTS:

PRES1 1

PARAMETERS :

$\begin{array}{llll}1.00000 \mathrm{E}-03 & 1.20000 \mathrm{E}-02 & 0.87000 & 1.0000\end{array}$

UNIT 15 TYPE 4

5 INPUTS:

FLOW 6 FLOW 5 PRES15 TEMP12 TEMP13

4 OUTPUTS :

FLOW 4 PRES13 PRES14 TEMP 4

PARAMETERS :

$1.10000 \mathrm{E}-02$

UNIT $16 \quad$ TYPE 5

3 INPUTS :

FLOW 5 PRES14 CTRL 8

1 OUTPUTS:

PRES 16

PARAMETERS :
$1.00000 \mathrm{E}-03$
$1.20000 \mathrm{E}-02$
0.87000
0.00000 
UNIT 17 TYPE 3

5 IN PUTS :

$\begin{array}{lllll}\text { PRES1 } 2 & \text { PRES16 TEMP13 } & \text { TEMP13 } & \text { TEMP13 }\end{array}$

2 OUTPUTS:

NULL 0 FLOW 5

PARAMETERS :

0.00000

0.00000

0.00000

0.00000

0.00000

$1.00000 E-03$

UNIT 18 TYPE 5

3 INPUTS :

FLOW 4 PRESI7 CTRL 9

1 OUTPUTS:

PRES 15

PARAMETERS :

$\begin{array}{llll}4.00000 E-02 & 4.00000 E-05 & 1.0000 & 0.00000\end{array}$

UNIT 19 TYPE 5

3 INPUTS:

FLOW 4 PRES18 CTRI 9

1 OUTPUTS :

PRES17

PARAME TERS :

$$
\begin{array}{llll}
1.20000 \mathrm{E}-02 & 1.20000 \mathrm{E}-05 & 1.0000 & 0.00000
\end{array}
$$

UNIT $20 \quad$ TYPE 5

3 IN PUTS :

FLOW 4 PRES19 CTRL 9

1 OUTPUTS :

PRES 18

PARAME TERS :

$\begin{array}{llll}1.20000 \mathrm{E}-02 & 1.20000 \mathrm{E}-05 & 1.0000 & 0.00000\end{array}$

UNIT 21 TYPE 1

4 IN PUTS :

FLOW 4 PRES20 RVPS 2 TEMP 5

3 OUTPUTS : 
PRES19 TEMP14 POWR 4

PARAMETERS :

$\begin{array}{lllll}3.6400 & 0.80100 & -0.19000 & -4.45000 \mathrm{E}-03 & 0.00000 \\ 0.00000 & 0.56400 & -8.62000 \mathrm{E}-02 & 0.00000 & 0.00000 \\ 0.31920 & 1.0000 & & & \end{array}$

UNIT 22 TYPE 2

5 INPUTS:

$\begin{array}{lllll}\text { FLOW } 4 & \text { PRES } 7 & \text { TEMP14 } & \text { TEMP13 } & \text { TEMP11 }\end{array}$

2 OUTPUTS :

TEMP11 PRES20

PARAMETERS :

2.0000

0.00000

\subsection{0}

240.00

50.000

0.11880

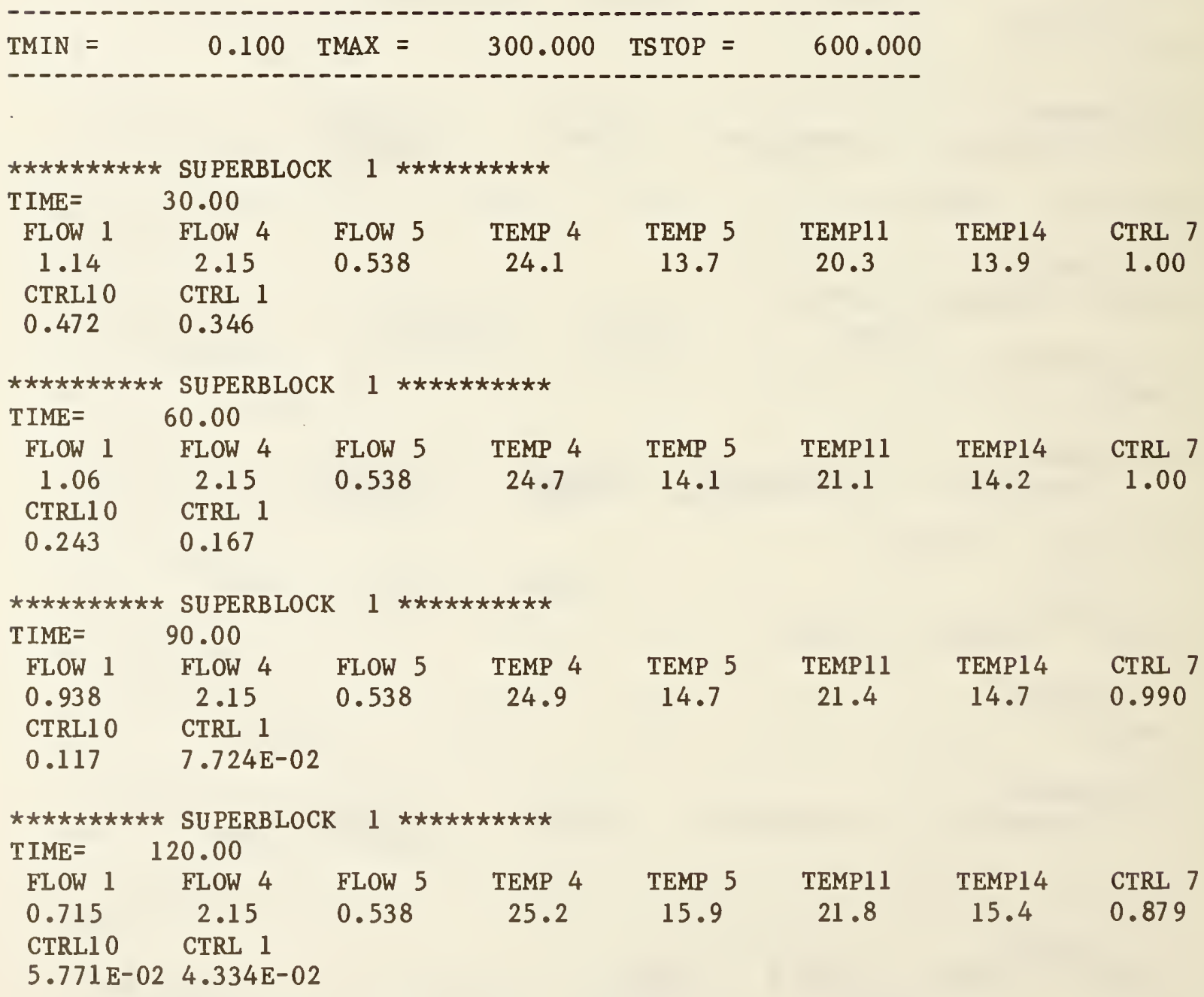




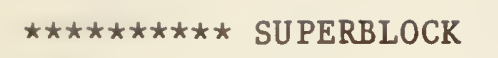

TIME $=150.00$

FLOW 1 FLOW 4 FLOW 5 TEMP $4 \quad$ TEMP 5 TEMP11 14 TEMP14 CTRL 7

$\begin{array}{lllllllll}0.670 & 2.15 & 0.538 & 25.5 & 16.6 & 22.2 & 16.8 & 0.835\end{array}$

CTRL10 CTRL 1

$5.049 \mathrm{E}-02 \quad 4.859 \mathrm{E}-02$

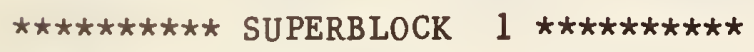

TIME $=180.00$

$\begin{array}{lclccccc}\text { FLOW 1 } & \text { FLOW 4 } & \text { FLOW 5 } & \text { TEMP 4 } & \text { TEMP 5 } & \text { TEMP11 } & \text { TEMP14 } & \text { CTRL } 7 \\ 0.611 & 2.15 & 0.538 & 25.8 & 16.9 & 22.6 & 16.9 & 0.781\end{array}$

$\begin{array}{lcl}0.611 & 2.15 \\ \text { CTRL10 CTRL } 1\end{array}$

4. $290 \mathrm{E}-023.355 \mathrm{E}-02$

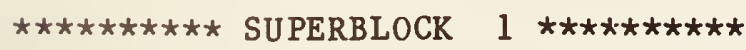

TIME $=210.00$

FLOW 1 FLOW 4 FLOW 5 TEMP 4. TEMP 5 TEMP11 4 $\begin{array}{lllllllll}0.565 & 2.15 & 0.538 & 26.1 & 17.5 & 23.0 & 17.6 & 0.734\end{array}$

CTRL10 CTRL 1

$3.766 \mathrm{E}-02 \quad 3.857 \mathrm{E}-02$

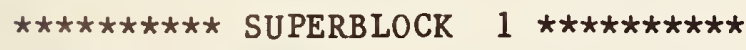

TIME $=240.00$

FLOW 1 FLOW 4 FLOW 5 TEMP $4 \quad$ TEMP $5 \quad$ TEMP11 4 TEMP14

$\begin{array}{llllllll}0.565 & 2.15 & 0.538 & 26.4 & 17.6 & 23.4 & 17.7 & 0.718\end{array}$

CTRL10 CTRL 1

$3.766 \mathrm{E}-02 \quad 4.034 \mathrm{E}-02$

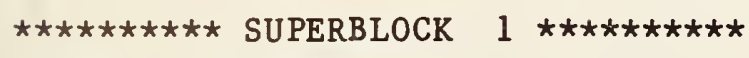

TIME $=270.00$

FLOW 1 FLOW 4 FLOW 5 TEMP $4 \quad$ TEMP $5 \quad$ TEMP11 4 TEMP14

$\begin{array}{lllllllll}0.565 & 2.15 & 0.538 & 26.6 & 17.6 & 23.7 & 17.7 & 0.708\end{array}$

CTRL10 CTRL 1

$3.766 \mathrm{E}-02 \quad 3.697 \mathrm{E}-02$

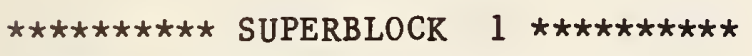

TIME $=300.00$

FLOW 1 FLOW 4 FLOW 5 TEMP 4 TEMP 5 TEMP11 $\quad$ TEMP14

$0.561 \quad 2.15$

$0.538 \quad 26.8$

17.7

23.9

17.8

0.698

CTRL10 CTRL 1

$3.723 E-02 \quad 3.358 E-02$

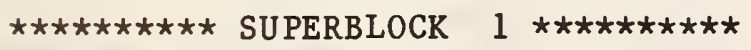

TIME $\quad 330.00$

FLOW 1 FLOW 4 FLOW 5 TEMP $4 \quad$ TEMP 5 TEMP11 4 TEMP14 CTRL 7

$0.550 \quad 2.15$

$0.538 \quad 26.9$

17.8

24.1

17.9

0.678

3.611E-02 $3.208 \mathrm{E}-02$

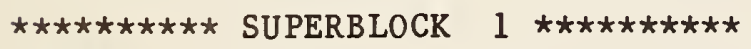

TIME $=360.00$

FLOW 1 FLOW 4 FLOW 5 TEMP $4 \quad$ TEMP 5 TEMPII $\quad$ TEMP14 


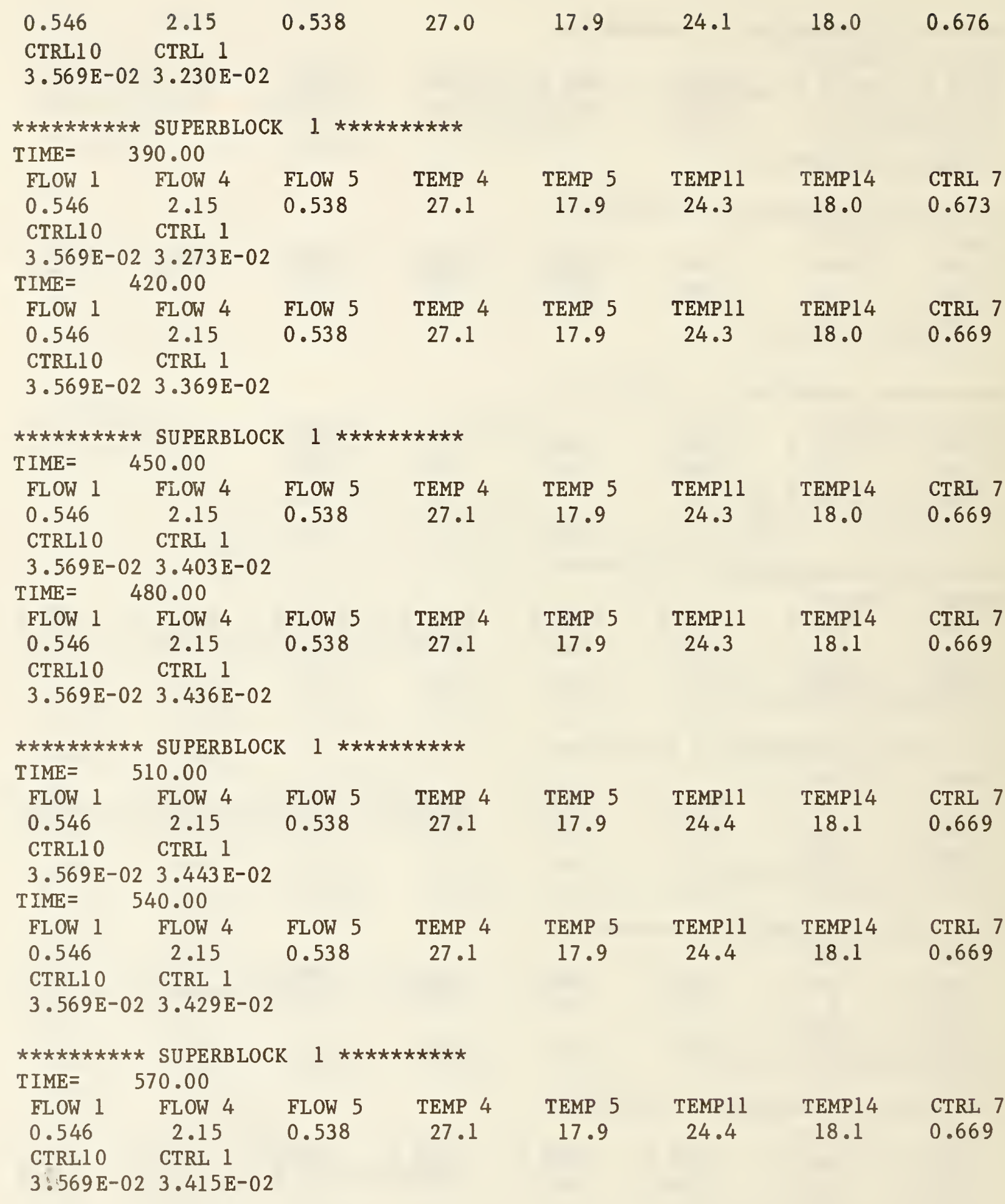




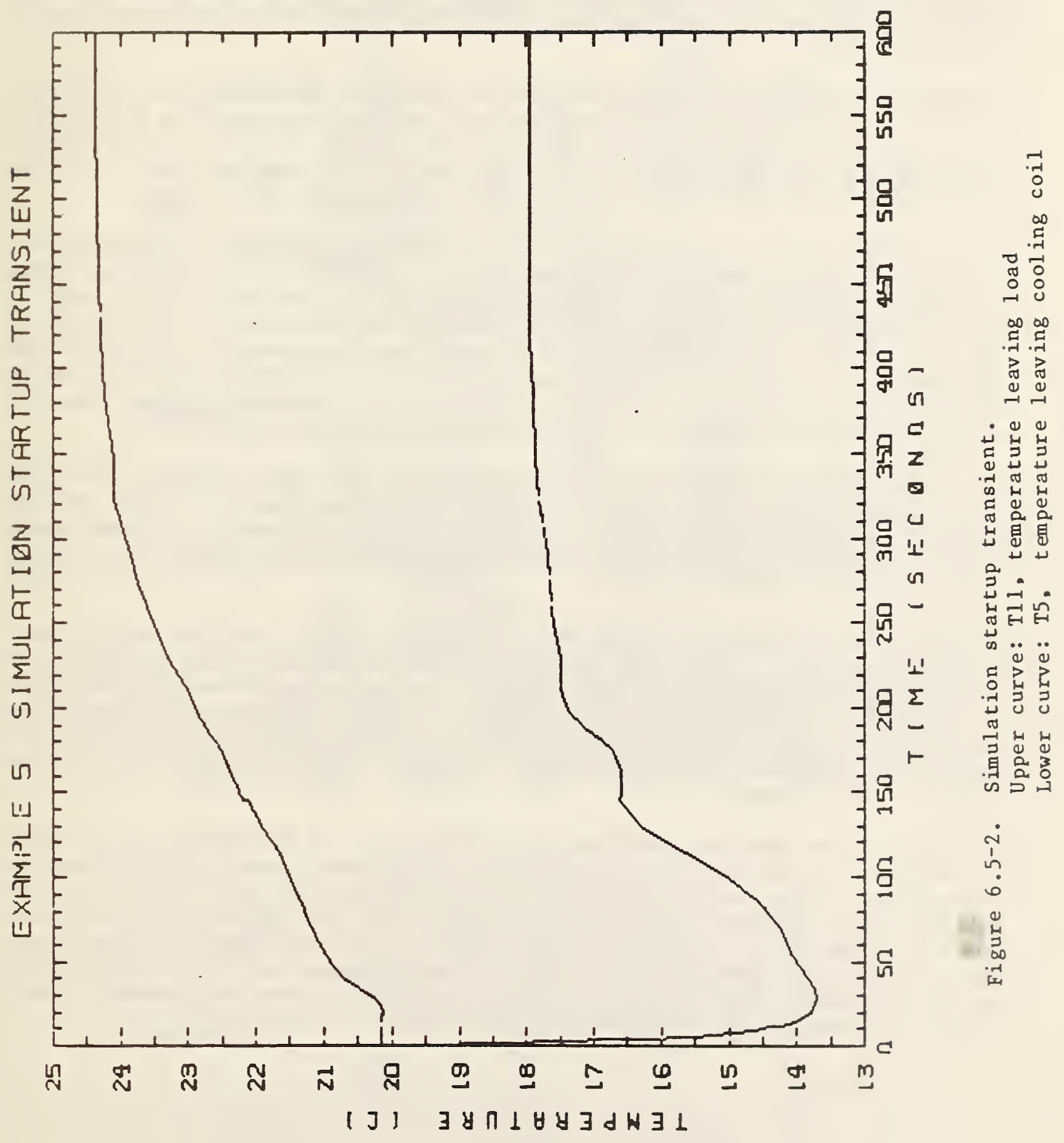




\subsection{Example 6: Single Zone Air Handler With Cooling Coil}

This example was generated by deleting the Unit 22, Type 2 component from Block 2 of Example 5, and adding a third block consisting of a supply duct, a conditioned zone, and a return duct. The three blocks are shown schematically in Figure 6.6-1.

After adding the third block, the initial value of the outdoor air temperature, T13, was changed to $25 \mathrm{C}$, and the new temperature variables T15 through T2 1 were all assigned initial values of $20 \mathrm{C}$. The fifth power variable, representing the heat flux through the zone walls, was set to $3 \mathrm{~kW}$. The reporting interval and 1 ist of reported variables were also changed.

The resulting simulation is an unmitigated disaster, failing to converge well on the first time step and terminating in a FORTRAN error (floating point overflow) shortly thereafter. The reported flow rate $W 4$ is less than zero at the first time step, suggesting that perhaps the equation solver (SNSQ) is unable to find a solution to the set of equations representing air flow rates and pressures. This set of equations is now divided between blocks 2 and 3 , making it more difficult to solve. Otherwise it is essentially the same set of equations as in Example 5, with only one additional pressure (P21).

On this assumption, Example 5 was run again for a few time steps and the first three significant digits of the final values of pressures P7 through P20 and flows W4 through W6 were entered as initial values in Example 6. Thus modified, the example ran smoothly. The configuration of this simulation is shown in Listing $6.6-1$.

The point to emphasize here is that dividing a tightly coupled set of equations into separate blocks (or superblocks) can sometimes cause problems. In this instance all that was required was a better set of initial guesses for the variables in question. In some cases it may be necessary to modify the block structure of a simulation to overcome similar problems. The Type 19 component, designed for passing flow streams between superblocks, can be useful in this regard.

The example was run for 86400 seconds ( 24 hours), and the final state of this run was used as the initial state of all subsequent runs. The outdoor air temperature, T13, and the heat flux through the zone wal1, Q5, were then declared time dependent boundary conditions, and the boundary variable file shown in Listing 6.6-2 and graphed in Figure 6.6-2 was prepared. Time zero, when the ambient temperature is lowest, corresponds to 5 A.M. Some results from a 24-hour simulation using these initial conditions and boundary conditions are presented in Figures 6.6-3 and 6.6-4. 
Example 6: AIR HANDLING UNIT WITH COOLING COIL AND CONDITIONED ZONE

Block 1

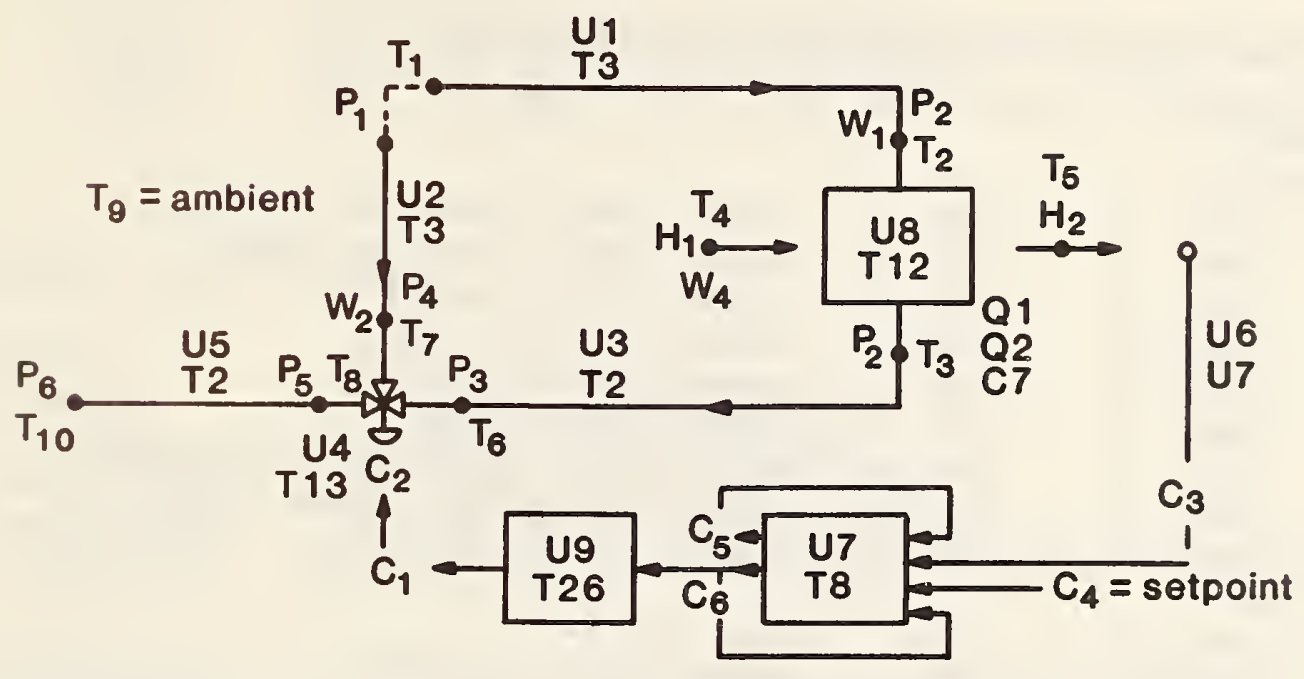

Block 2

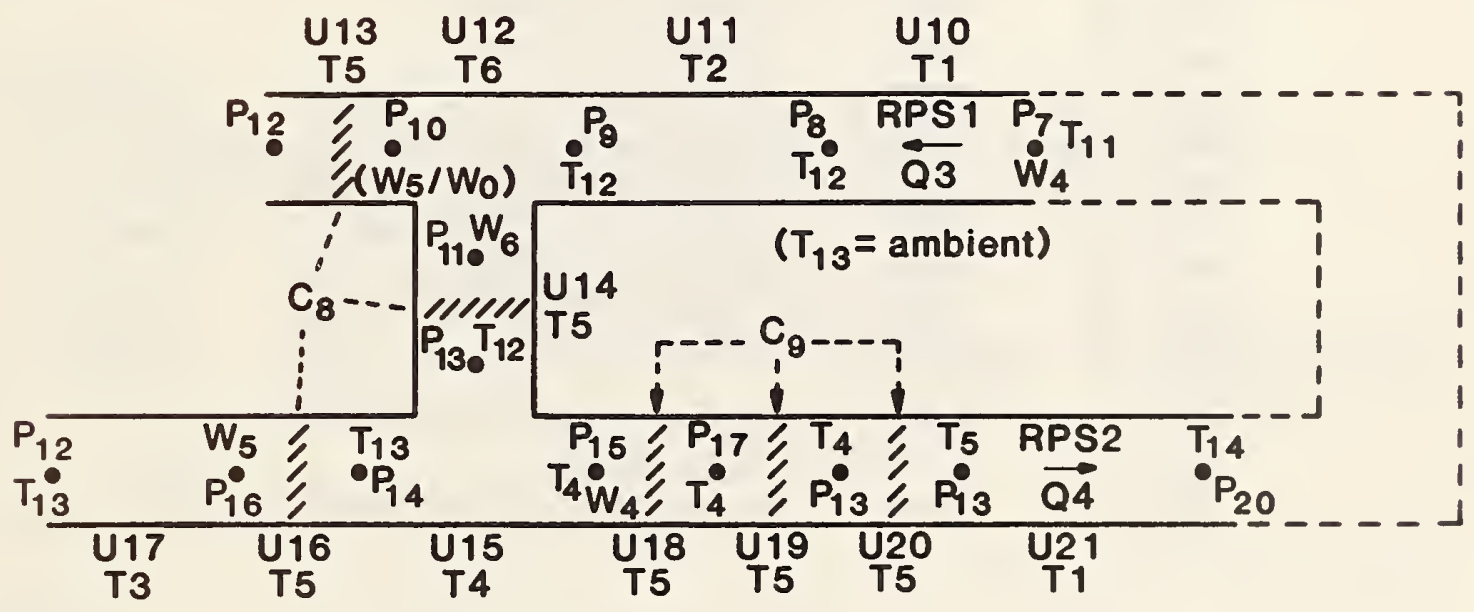

Block 3

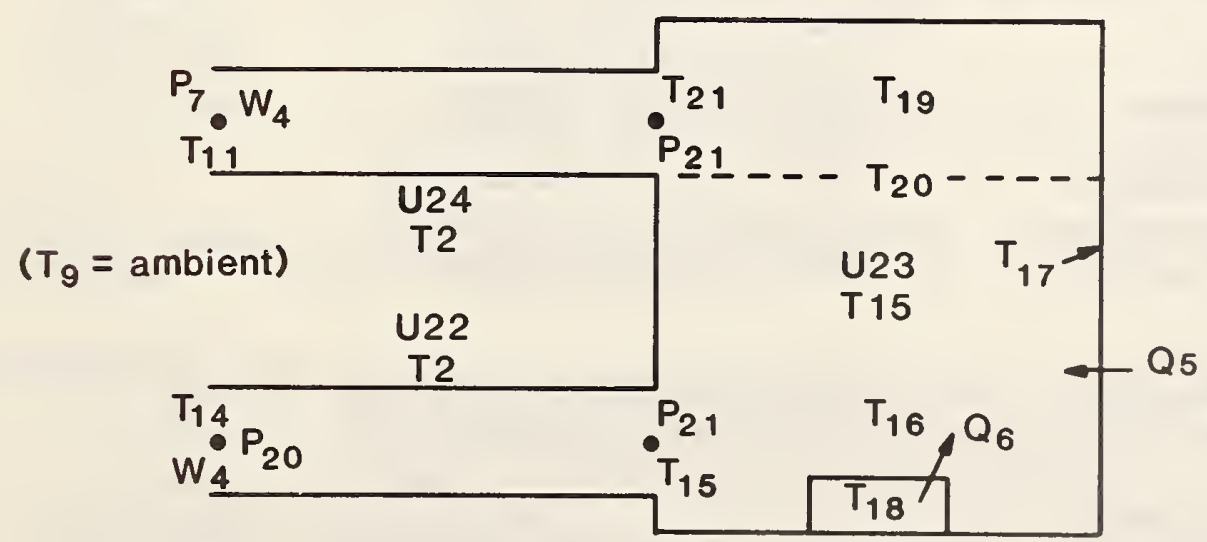

Figure 6.6-1. System schematic: single zone air handler with cooling coil. 
Listing 6.6-1. Simulation Configuration

AIR HANDLER WITH COOLING COIL AND CONDITIONED ZONE

SUPERBLOCK 1

BLOCK 1

UNIT 1 TYPE 3 - INLET CONDUIT (DUCT OR PIPE)

UNIT 2 TYPE 3 - INLET CONDUIT (DUCT OR PIPE)

UNIT 3 TYPE 2 - CONDUIT (DUCT OR PIPE)

UNIT 4 TYPE 13 - THREE-WAY VALVE MODEL

UNIT 5 TYPE 2 - CONDUIT (DUCT OR PIPE)

UNIT 6 TYPE 7 - TEMPERATURE SENSOR

UNIT 7 TYPE 8 - PROPORTIONAL-INTEGRAL CONTROLLER

UNIT 8 TYPE 12 - COOLING OR DEHUMIDIFYING COIL

BLOCK 2

TYPE 26 - CONTROL SIGNAL INVERTER

UNIT 10 TYPE 1 - FAN OR PUMP

UNIT 11 TYPE 2 - CONDUIT (DUCT OR PIPE)

UNIT 12 TYPE 6 - FLOW SPLIT

UNIT 13 TYPE 5 - DAMPER OR VALVE

UNIT 14 TYPE 5 - DAMPER OR VALVE

UNIT 15 TYPE 4 - FLOW MERGE

UNIT 16 TYPE 5 - DAMPER OR VALVE

UNIT 17 TYPE 3 - INLET CONDUIT (DUCT OR PIPE)

UNIT 18 TYPE 5 - DAMPER OR VALVE

UNIT 19 TYPE 5 - DAMPER OR VALVE

UNIT 20 TYPE 5 - DAMPER OR VALVE

UNIT 21 TYPE 1 - FAN OR PUMP

BLOCK 3

UNIT 22 TYPE 2 - CONDUIT (DUCT OR PIPE)

UNIT 23 TYPE 15 - ROOM MODEL

UNIT 24 TYPE 2 - CONDUIT (DUCT OR PIPE)

UNIT 1 TYPE 3

INLET CONDUIT (DUCT OR PIPE)

1 INPUTS :

PRESSURE

PRESSURE

TEMPERATURE

TEMPERATURE

TEMPERATURE

1 - INLET FLUID PRESSURE

2 - OUTLET FLUID PRESSURE

1 - INLET FLUID TEMPERATURE

9 - AMBIENT AIR TEMPERATURE

2 - OUTLET FLUID TEMPERATURE (SAME AS FIRST OUTPUT)

2 OUTPUTS :

TEMPERATURE

FLOW

2 - OUTLET FLUID TEMPERATURE (SAME AS FIFTH INPUT)

1 - FLUID MASS FLOW RATE

3 PARAMETERS:

2.97000 INSIDE HEAT TRANSFER COEFFICIENT X AREA (KW/C) 


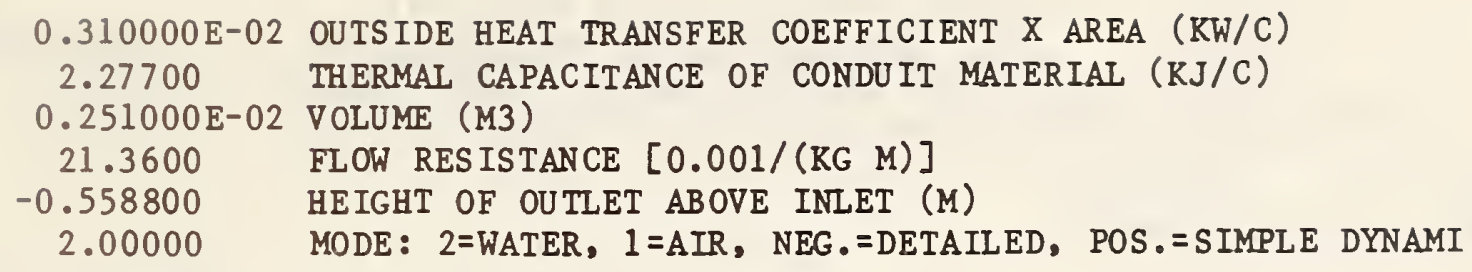

1 INPUTS :

$\begin{array}{ll}\text { PRESSURE } & 1 \text { - INLET FLUID PRESSURE } \\ \text { PRESSURE } & 4 \text { - OUTLET FLUID PRESSURE } \\ \text { TEMPERATURE } & 1 \text { - INLET FLUID TEMPERATURE } \\ \text { TEMPERATURE } & 9 \text { - AMBIENT AIR TEMPERATURE } \\ \text { TEMPERATURE } & 7 \text { - OUTLET FLUID TEMPERATURE (SAME AS FIRST OUTPUT) }\end{array}$

2 OUTPUTS :

TEMPERATURE 7 - OUTLET FLUID TEMPERATURE (SAME AS FIFTH INPUT) FLOW

3 PARAMETERS :
0.276000
$0.290000 \mathrm{E}-03$
INSIDE HEAT TRANSFER COEFFICIENT X AREA (KW/C)
0.211600
$0.230000 \mathrm{E}-03$
OUTSIDE HEAT TRANSFER COEFFICIENT X AREA (KW/C)
0.230000 E-03 VOLUME (M3)
14.8000 FLOW RESISTANCE $[0.001 /($ KG M)]
-0.558800 HEIGHT OF OUTLET ABOVE INLET (M)
2.00000 MODE: 2=WATER, 1=AIR, NEG.=DETAILED, POS .=SIMPLE DYNAMI

UNIT 3 TYPE 2

CONDUIT (DUCT OR PIPE)

1 INPUTS :
FLOW
PRESSURE
1 - FLUID MASS FLOW RATE
TEMPERATURE
3 - OUTLET PRESSURE
TEMPERATURE
3 - FLUID INLET TEMPERATURE
TEMPERATURE
9 - AMBIENT TEMPERATURE
6 - OUTLET FLUID TEMPERATURE (SAME AS FIRST OUTPUT)

2 OUTPUTS :

TEMPERATURE 6 - OUTLET FLUID TEMPERATURE (SAME AS FIFTH INPUT)

PRESSURE

2 - INLET PRESSURE

3 PARAMETERS:

2.70000 INSIDE HEAT TRANSFER COEFFICIENT X AREA (KW/C)

0.281000E-02 OUTSIDE HEAT TRANSFER COEFFICIENT X AREA (KW/C)

2.07000 THERMAL CAPACITANCE OF CONDUIT MATERIAL (KJ/C)

0.227800 E-02 VOLUME (M3)

11.9000 FLOW RESISTANCE [0.001/(KG M)] 
0.000000 HEIGHT OF OUTLET ABOVE INLET (M)

2.00000 MODE: 2=WATER, 1=AIR, NEG.=DETAILED,POS =SIMPLE DYNAMI

UNIT 4 TYPE 13

THREE-WAY VALVE MODEL

1 IN PUTS :

FLOW

FLOW

PRESSURE

TEMPERATURE

TEMPERATU RE

CONTROL

CONTROL

1 - INLET MASS FLOW RATE, PORT 1 (OPEN WHEN CONTROL = 1

2 - INLET MASS FLOW RATE, PORT 2 (OPEN WHEN CONTROL = 0

5 - OUTLET PRESSURE

6 - INLET TEMPERATURE, PORT 1

7 - INLET TEMPERATURE, PORT 2

1 - CONTROL VARIABLE FROM CONTROLLER

2 - ACTUATOR RELATIVE POSITION (SAME AS OUTPUT 1)

2 OUTPUTS :

CONTROL

FLOW

PRESSURE

PRESSURE

TEMPERATURE

CONTROL

2 - ACTUATOR RELATIVE POSITION

3 - OUTLET MASS FLOWRATE

3 - INLET PRESS., PORT 1 (OPEN $\Rightarrow$ CONTROL VARIABLE = 1)

4 - INLET PRESS., PORT 2 (OPEN $\Rightarrow$ CONTROL VARIABLE $=0$ )

8 - OUTLET TEMPERATURE

10 - VALVE STEM RELATIVE POSITION

3 PARAMETERS :

0.300000 PORT 1 FLOW RESISTANCE, PORT 1 OPEN (CTRL=1)[0.001/(KG

1.50000 PORT 2 FLOW RESISTANCE, PORT 2 OPEN (CTRL=0)[0.001/(KG

0.400000E-02 LEAKAGE PARAMETER (DIMENS IONLESS)

10.0000 ACTUATOR TIME CONSTANT (SEC)

0.100000 HYSTERESIS PARAMETER (DIMENSIONLESS)

0.000000 PORTS 1.2 MODE: $0=>\mathrm{LIN} / \mathrm{LIN}, 1=>\mathrm{EXP} / \mathrm{LIN}, \mathrm{ELSE}=>\mathrm{EXP} / \mathrm{EXP}$

UNIT 5 TYPE 2

CONDUIT (DUCT OR PIPE)

1 INPUTS :

FLOW

PRESSURE

TEMPERATURE

TEMPERATURE

TEMPERATURE

3 - FLUID MASS FLOW RATE

6 - OUTLET PRESSURE

8 - FLUID INLET TEMPERATURE

9 - AMBIENT TEMPERATURE

10 - OUTLET FLUID TEMPERATURE (SAME AS FIRST OUTPUT)

2 OUTPUTS:

TEMPERATURE 10 - OUTLET FLUID TEMPERATURE (SAME AS FIFTH INPUT)

PRESSURE

5 - INLET PRESSURE

3 PARAMETERS :

38.0000

INSIDE HEAT TRANSFER COEFFICIENT X AREA (KW/C)

0.440000E-01 OUTSIDE HEAT TRANSFER COEFFICIENT X AREA (KW/C)

32.2000 THERMAL CAPACITANCE OF CONDUIT MATERIAL (KJ/C)

$0.355000 \mathrm{E}-01$ VOLUME (M3) 


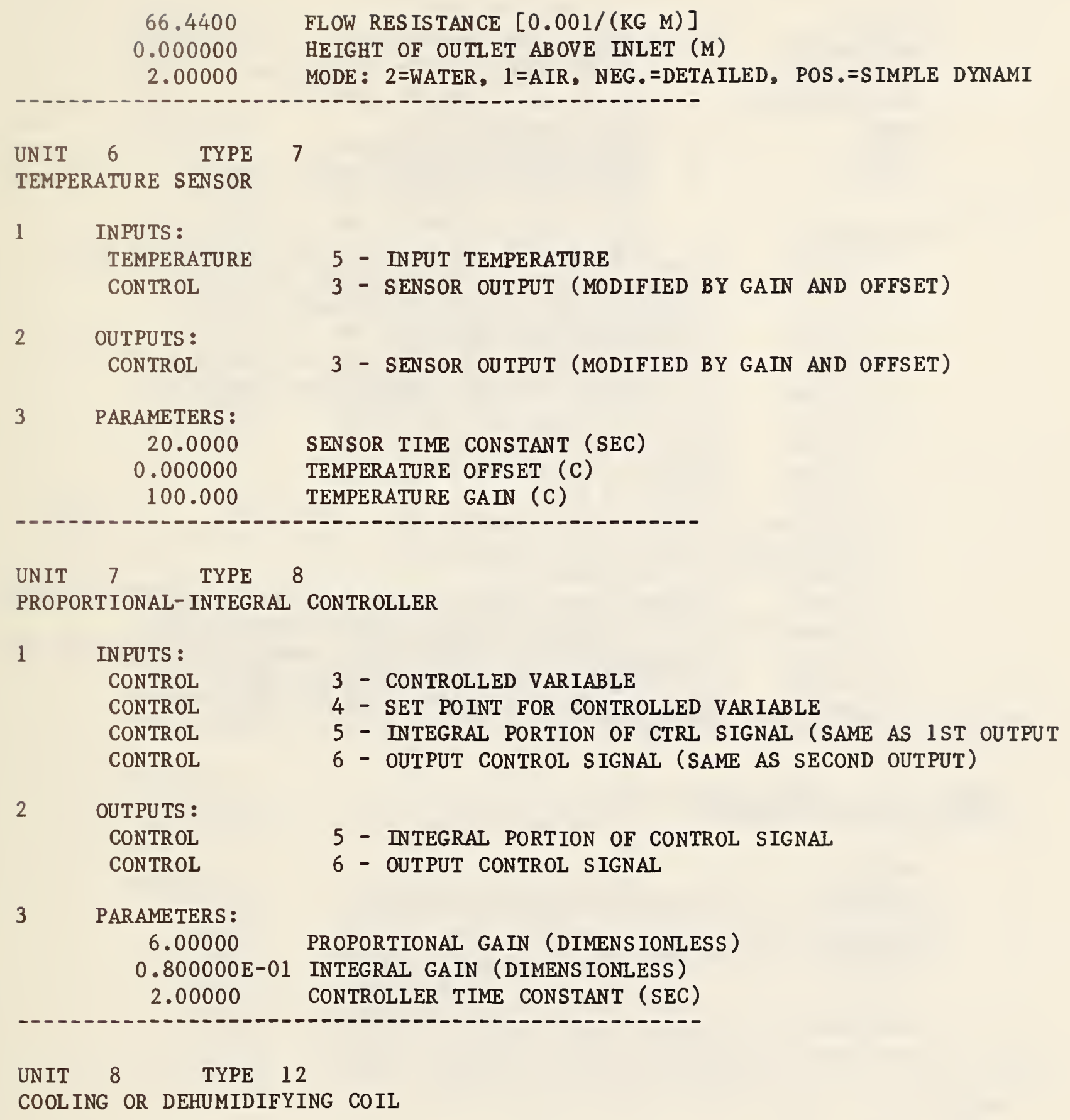

$\begin{array}{llll}\text { UNIT } & 8 & \text { TYPE } & 12\end{array}$

COOLING OR DEHUMIDIFYING COIL

1 INPUTS :
FLOW
TEMPERATURE
FLOW
1 - WATER MASS FLOW RATE
TEMPERATURE
2 - INLET WATER TEMPERATURE
ABS . HUMIDITY
4 - DRY AIR MASS FLOW RATE
4 - INLET DRY BULB AIR TEMPERATURE
TEMPERATURE
1 - INLET AIR HUMIDITY RATIO
TEMPERATURE
3 - OUTLET WATER TEMPERATURE (SAME AS FIRST OUTPUT)
ABS. HUMIDITY
5 - OUTLET AIR TEMPERATURE (SAME AS SECOND OUTPUT)
2 - OUTLET AIR HUMIDITY RATIO (SAME AS THIRD OUTPUT) 
OUTPUTS :

TEMPERATURE

3 - OUTLET WATER TEMPERATURE TEMPERATURE

5 - OUTLET AIR DRY BULB TEMPERATURE

ABS. HUMIDITY

2 - OUTLET AIR HUMIDITY RATIO

POWER

POWER

1 - TOTAL COOL ING LOAD

CONTROL

2 - SENSIBLE COOL ING LOAD

7 - WET FRACTION OF COIL SURFACE AREA

PARAMETERS:

0.000000

6.23810

COIL TYPE: 0=FLAT CONTINUOUS FINS, $1=$ CIRCULAR FINS

101.715

PRIMARY (TUBE EXTERIOR) SURFACE AREA (M2)

6.20000

SECONDARY (FIN) SURFACE AREA (M2)

0.416000

INTERNAL SURFACE AREA (M2)

0.204000

RATIO OF MINIMUM AIR FLOW AREA TO FACE AREA ( - )

0.722600 FIN MATERIAL THERMAL CONDUCTIVITY [KW/(M K)]

5.51180 COIL FACE AREA (M2)

16.0000

NUMBER OF FINS PER CENTIMETER

6.00000

NUMBER OF TUBES PER ROW

$0.158800 \mathrm{E}-01$ OUTSIDE TUBE DIAMETER (M)

$0.144500 \mathrm{E}-01$ INSIDE TUBE DIAMETER (M)

0.150000 E-03 FIN THICKNESS (M)

34.6690

THERMAL CAPACITANCE (MASS X SPECIFIC HEAT) OF COIL (KJ/

$0.259000 \mathrm{E}-01$

0.508000

0.165100

TUBE ROW SPACING IN AIR FLOW DIRECTION (M)

0.386000

FIN DIAMETER [IF PAR(1)=1] OR LENGTH $[\operatorname{IF} \operatorname{PAR}(1)=0](M)$

COIL DEPTH IN AIR FLOW DIRECTION (M)

TUBE THERMAL CONDUCTIVITY [KW/(M K)]

UNIT 9 TYPE 26

CONTROL SIGNAL INVERTER

1 INPUTS :

CONTROL $6-$ INPUT CONTROL SIGNAL

2 OUTPUTS:

CONTROL 1 - OUTPUT CONTROL SIGNAL

3 PARAMETERS :

UNIT $10 \quad$ TYPE 1

FAN OR PUMP

1 INPUTS :

$\begin{array}{lrl}\text { FLOW } & 4 \text { - MASS FLOW RATE OF FLUID } \\ \text { PRESSURE } & 8 \text { - OUTLET PRESSURE } \\ \text { RVPS } & 1 \text { - FAN OR PUMP ROTATIONAL SPEED } \\ \text { TEMPERATURE } & 11 \text { - INLET FLUID TEMPERATURE }\end{array}$

2 OUTPUTS : 


$\begin{array}{lrl}\text { PRESSURE } & 7 \text { - INLET PRESSURE } \\ \text { TEMPERATURE } & 12 \text { - OUTLET FLUID TEMPERATURE } \\ \text { POWER } & 3 \text { - POWER CONSUMPTION }\end{array}$

PARAMETERS:

3.64000 1ST PRESSURE COEFFICIENT

0.801000 2ND PRESSURE COEFFICIENT

-0.190000 3RD PRESSURE COEFFICIENT

$-0.445000 \mathrm{E}-02$ 4TH PRESSURE COEFFICIENT

0.000000 5TH PRESSURE COEFFICIENT

0.000000 1ST EFFICIENCY COEFFICIENT

0.564000 2ND EFFICIENCY COEFFICIENT

$-0.862000 \mathrm{E}-01$ 3RD EFFICIENCY COEFFICIENT

0.000000 4TH EFFICIENCY COEFFICIENT

$0.000000 \quad 5 \mathrm{TH}$ EFFICIENCY COEFFICIENT

0.319200 DIAMETER (M)

1.00000 MODE : AIR $=1$, WATER $=2$

UNIT 11 TYPE 2

CONDUIT (DUCT OR PIPE)

1 INPUTS :

FLOW 4 - FLUID MASS FLOW RATE

PRESSURE 9 - OUTLET PRESSURE

TEMPERATURE 12 - FLUID INLET TEMPERATURE

TEMPERATURE 12 - AMBIENT TEMPERATURE

TEMPERATURE 12 - OUTLET FLUID TEMPERATURE (SAME AS FIRST OUTPUT)

2 OUTPUTS :

TEMPERATURE 0 - OUTLET FLUID TEMPERATURE (SAME AS FIFTH INPUT)

PRESSURE 8 - INLET PRESSURE

3 PARAMETERS:

0.000000 INSIDE HEAT TRANSFER COEFFICIENT X AREA (KW/C)

0.000000 OUTSIDE HEAT TRANSFER COEFFICIENT X AREA (KW/C)

0.000000 THERMAL CAPACITANCE OF CONDU IT MATERIAL (KJ/C)

$0.000000 \quad$ VOLUME (M3)

$0.100000 \mathrm{E}-01$ FLOW RESISTANCE $[0.001 /(\mathrm{KG} \mathrm{M})]$

0.000000 HEIGHT OF OUTLET ABOVE INLET (M)

1.00000 MODE: 2=WATER, 1=AIR, NEG.=DETAILED, POS.=SIMPLE DYNAMI

UNIT 12 TYPE 6

FLOW SPLIT

1 INPUTS :

FLOW

PRESSURE

4 - INLET MASS FLOW RATE

PRESSURE

10 - OUTLET PRESSURE 1

11 - OUTLET PRESSURE 2

2 OUTPUTS : 


$\begin{array}{ll}\text { FLOW } & 0 \text { - OUTLET MASS FLOW RATE } 1 \\ \text { FLOW } & 6 \text { - OUTLET MASS FLOW RATE } 2 \\ \text { PRESSURE } & 9 \text { - INLET PRESSURE }\end{array}$

PARAMETERS :

0.110000 E-01 FLOW RESISTANCE [0.001/(KG M)]

UNIT 13 TYPE 5

DAMPER OR VALVE

1 INPUTS :

$\begin{array}{lrl}\text { FLOW } & 5 \text { - FLUID MASS FLOW RATE } \\ \text { PRESSURE } & 12 \text { - OUTLET PRESSURE } \\ \text { CONTROL } & 8 \text { - CONTROL: RELATIVE POSITION OF DAMPER OR VALVE ( - ) }\end{array}$

2 OUTPUTS :

PRESSURE $\quad 10$ - INLET PRESSURE

3 PARAMETERS:

0.100000 E-02 FLOW RESISTANCE, DAMPER OR VALVE OPEN $[0.001 /(\mathrm{KG} \mathrm{M})]$

0.120000 E-01 LEARAGE PARAMETER (DIMENSIONLESS)

0.870000 CHARACTERISTIC: $0=>E X P ., 1=>L$ IN. , INTERMEDIATE $=>$ INTERME

0.000000 MODE : $0=>$ CLOSED WHEN CONTROL $=0 ; 1=>$ CLOSED WHEN CONTROL $=$

UNIT 14 TYPE 5

DAMPER OR VALVE

1 INPUTS :

FLOW

PRESSURE

CONTROL
6 - FLUID MASS FLOW RATE

13 - OUTLET PRESSURE

8 - CONTROL: RELATIVE POSITION OF DAMPER OR VALVE ( - )

2 OUTPUTS :

PRESSURE 11 - INLET PRESSURE

3 PARAMETERS:

0.100000 E-02 FLOW RESISTANCE, DAMPER OR VALVE OPEN $[0.001 /(\mathrm{KG} \mathrm{M})]$

$0.120000 \mathrm{E}-01$ LEAKAGE PARAMETER (DIMENS IONLESS)

0.870000 CHARACTERISTIC: $0=>$ EXP., $1=>\mathrm{LIN} .$, INTERMEDIATE $=>$ INTERME

1.00000 MODE: $0=>$ CLOSED WHEN CONTROL $=0 ; 1=>$ CLOSED WHEN CONTROL=

UNIT 15 TYPE 4

FLOW MERGE

1 INPUTS :

$\begin{array}{lrl}\text { FLOW } & 6 \text { - INLET MASS FLOW RATE } 1 \\ \text { FLOW } & 5 \text { - INLET MASS FLOW RATE } 2 \\ \text { PRESSURE } & 15 \text { - OUTLET PRESSURE } \\ \text { TEMPERATURE } & 12 \text { - INLET TEMPERATURE } 1\end{array}$


TEMPERATURE 13 - INLET TEMPERATURE 2

2 OUTPUTS:

FLOW

PRESSURE

PRESSURE

TEMPERATURE

$$
\begin{array}{r}
4 \text { - OUTLET MASS FLOW RATE } \\
13 \text { - INLET PRESSURE } 1 \\
14 \text { - INLET PRESSURE } 2 \\
4 \text { - OUTLET TEMPERATURE }
\end{array}
$$

3

PARAMETERS :

$0.110000 \mathrm{E}-01$ FLOW RESISTANCE $[0.001 /($ KG M)]

UNIT 16 TYPE 5

DAMPER OR VALVE

1 IN PUTS :

FLOW

PRESSURE

5 - FLUID MASS FLOW RATE

CONTROL

14 - OUTLET PRESSURE

8 - CONTROL: RELATIVE POSITION OF DAMPER OR VALVE ( - )

2 OUTPUTS:

PRESSURE 16 - INLET PRESSURE

3 PARAMETERS:

0.100000 E-02 FLOW RESISTANCE, DAMPER OR VALVE OPEN $[0.001 /(\mathrm{KG} \mathrm{M})]$

$0.120000 \mathrm{E}-01$ LEAKAGE PARAMETER (DIMENSIONLESS)

0.870000 CHARACTERISTIC: $0=>$ EXP., $1=>$ L IN., INTERMEDIATE $=>$ INTERME

0.000000 MODE : $0=>$ CLOSED WHEN CONTROL $=0 ; 1=>$ CLOSED WHEN CONTROL $=$

UNIT 17 TYPE 3

INLET CONDUIT (DUCT OR PIPE)

1 INPUTS:

$\begin{array}{ll}\text { PRESSURE } & 12 \text { - INLET FLUID PRESSURE } \\ \text { PRESSURE } & 16 \text { - OUTLET FLUID PRESSURE } \\ \text { TEMPERATURE } & 13 \text { - INLET FLUID TEMPERATURE } \\ \text { TEMPERATURE } & 13 \text { - AMBIENT AIR TEMPERATURE } \\ \text { TEMPERATURE } & 13 \text { - OUTLET FLUID TEMPERATURE (SAME AS FIRST OUTPUT) }\end{array}$

2 OUTPUTS :

TEMPERATURE 0 - OUTLET FLUID TEMPERATURE (SAME AS FIFTH INPUT)

FLOW

5 - FLUID MASS FLOW RATE

3 PARAMETERS:

0.000000

0.000000

0.000000

0.000000

0.100000 E-02 FLOW RESISTANCE $[0.001 /$ (KG M)]

0.000000 HEIGHT OF OUTLET ABOVE INLET (M)

1.00000

INSIDE HEAT TRANSFER COEFFICIENT X AREA (KW/C) OUTSIDE HEAT TRANSFER COEFFICIENT X AREA (KW/C) THERMAL CAPACITANCE OF CONDUIT MATERIAL (KJ/C) VOLUME (M3)

MODE : 2=WATER, 1=AIR, NEG.=DETAILED, POS .=SIMPLE DYNAMI 
UNIT 18 TYPE 5

DAMPER OR VALVE

1 INPUTS:

FLOW

PRESSURE

4 - FLUID MASS FLOW RATE

CONTROL

17 - OUTLET PRESSURE

9 - CONTROL: RELATIVE POSITION OF DAMPER OR VALVE ( - )

2 OUTPUTS :

PRESSURE 15 - INLET PRESSURE

3 PARAMETERS:

$0.400000 \mathrm{E}-01$ FLOW RESISTANCE, DAMPER OR VALVE OPEN $[0.001 /(\mathrm{KG} \mathrm{M})]$

0.400000 E-0 4 LEAKAGE PARAMETER (DIMENS IONLESS)

1.00000 CHARACTERISTIC: $0=>$ EXP., $1=>$ L IN., INTERMEDIATE $=>$ INTERME

0.000000 MODE: $0=>$ CLOSED WHEN CONTROL $=0 ; 1=>$ CLOSED WHEN CONTROL=

UNIT 19 TYPE 5

DAMPER OR VALVE

1 INPUTS :

FLOW 4 - FLUID MASS FLOW RATE

PRESSURE 18 - OUTLET PRESSURE

CONTROL

9 - CONTROL: RELATIVE POSITION OF DAMPER OR VALVE ( - )

2 OUTPUTS :

PRESSURE 17 - INLET PRESSURE

3 PARAMETERS :

$0.120000 E-01$ FLOW RESISTANCE, DAMPER OR VALVE OPEN $[0.001 /(\mathrm{KG} \mathrm{M})]$

0.120000 E-04 LEAKAGE PARAMETER (DIMENS IONLESS)

1.00000 CHARACTERISTIC: $0=>$ EXP., $1=>$ L IN., INTERMEDIATE $=>$ INTERME

0.000000 MODE : $0=>$ CLOSED WHEN CONTROL $=0 ; 1=>$ CLOSED WHEN CONTROL=

UNIT $20 \quad$ TYPE 5

DAMPER OR VALVE

1 INPUTS :

FLOW

PRESSURE

CONTROL

4 - FLUID MASS FLOW RATE

19 - OUTLET PRESSURE

9 - CONTROL: RELATIVE POSITION OF DAMPER OR VALVE ( - )

2 OUTPUTS :

PRESSURE 18 - INLET PRESSURE

3 PARAMETERS:

$0.120000 E-01$ FLOW RESISTANCE. DAMPER OR VALVE OPEN $[0.001 /(\mathrm{KG} \mathrm{M})]$

0.120000 E-04 LEAKAGE PARAMETER (DIMENS IONLESS) 
1.00000 CHARACTERISTIC: $0=>$ EXP. $1=>\mathrm{LIN}$. , INTERMEDIATE $=>$ INTERME 0.000000 MODE : $0=>C L O S E D$ WHEN CONTROL $=0 ; 1=>C L O S E D$ WHEN CONTROL $=$

UNIT 21 TYPE 1

FAN OR PUMP

1 INPUTS :

FLOW

PRESSURE

RVPS

TEMPERATURE

4 - MASS FLOW RATE OF FLUID

20 - OUTLET PRESSURE

2 - FAN OR PUMP ROTATIONAL SPEED

5 - INLET FLUID TEMPERATURE

2 OUTPUTS :

$\begin{array}{lrl}\text { PRESSURE } & 19 \text { - INLET PRESSURE } \\ \text { TEMPERATURE } & 14 \text { - OUTLET FLUID TEMPERATURE } \\ \text { POWER } & 4 \text { - POWER CONSUMPTION }\end{array}$

3 PARAMETERS:

3.64000
0.801000

0.801000

1ST PRESSURE COEFFICIENT

$-0.190000$

2ND PRESSURE COEFFICIENT

3RD PRESSURE COEFFICIENT

$-0.445000 \mathrm{E}-02$

0.000000

4 TH PRESSURE COEFFICIENT

0.000000

$5 \mathrm{TH}$ PRESSURE COEFFICIENT

0.564000

1ST EFFICIENCY COEFFICIENT

$-0.862000 E-01$

2ND EFFICIENCY COEFFICIENT

0.000000

0.000000

0.319200

3RD EFFICIENCY COEFFICIENT

4TH EFFICIENCY COEFFICIENT

5 TH EFFICIENCY COEFFICIENT

1.00000 DIAMETER (M)

MODE : $A I R=1$, WATER $=2$

UNIT 22 TYPE 2

CONDUIT (DUCT OR PIPE)

1 IN PUTS :

FLOW

PRESSURE

4 - FLUID MASS FLOW RATE

TEMPERATURE

TEMPERATURE

21 - OUTLET PRESSURE

TEMPERATURE

14 - FLUID INLET TEMPERATURE

9 - AMBIENT TEMPERATURE

15 - OUTLET FLUID TEMPERATURE (SAME AS FIRST OUTPUT)

2 OUTPUTS :

TEMPERATURE PRESSURE

15 - OUTLET FLUID TEMPERATURE (SAME AS FIFTH INPUT) 20 - INLET PRESSURE

3 PARAMETERS:

1.00000

0.250000

220.000

7.75000

INSIDE HEAT TRANSFER COEFFICIENT X AREA (KW/C) OUTSIDE HEAT TRANSFER COEFFICIENT X AREA (KW/C) THERMAL CAPACITANCE OF CONDUIT MATERIAL $(\mathrm{KJ} / \mathrm{C})$ VOLUME (M3) 
$0.600000 \mathrm{E}-01$ FLOW RESISTANCE [0.001/(KG M)]

0.000000 HEIGHT OF OUTLET ABOVE INLET (M)

1.00000 MODE: 2=WATER, 1=AIR, NEG。=DETAILED, POS.=SIMPLE DYNAMI

UNIT 23 TYPE 15

ROOM MODEL

1 INPUTS :

FLOW

TEMPERATURE

TEMPERATURE

TEMPERATURE

TEMPERATURE

TEMPERATURE

POWER

POWER
4 - MASS FLOW RATE OF VENTILATION AIR
15 - VENTILATION AIR INLET TEMPERATURE
16 - TEMP., FULLY MIXED PART OF AIR MASS [SAME AS OUT(1)
17 - WAII MASS TEMPERATURE (SAME AS SECOND OUTPUT)
18 - INTERIOR MASS TEMPERATURE (SAME AS THIRD OUTPUT)
19 - SPATIAL AVG. TEMP., PISTON FLOW AIR MASS (OUTPUT 4)
5 - CONDUCTION HEAT FLOW INTO WALL MASS
6 - HEAT FLOW DUE TO INTERNAL GAINS

2 OUTPUTS :

TEMPERATURE

TEMPERATURE

TEMPERATURE

TEMPERATURE

TEMPERATURE

TEMPERATURE

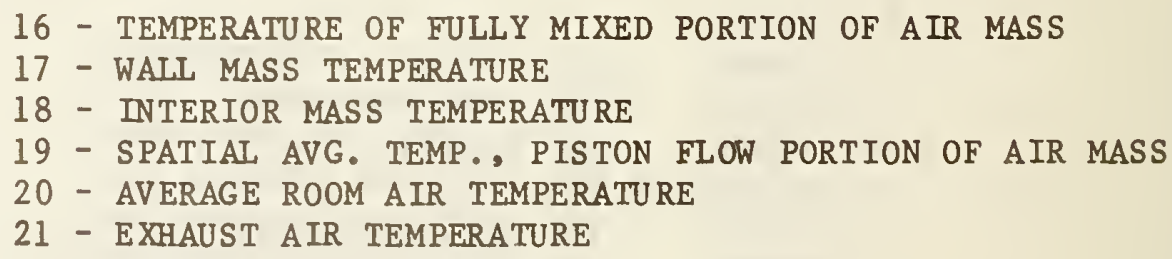

3 PARAMETERS:

1440.00 VOLUME OF ROOM AIR MASS (M3)

40000.0 THERMAL CAPACITANCE OF WALLS ( $\mathrm{KJ} / \mathrm{C}$ )

4000.00 THERMAL CAPACITANCE OF INTERIOR MASS (KJ/C)

1.00000 HEAT TRANSFER COEFF. TIMES AREA FOR WALL MASS (KW/C)

0.200000 HEAT TRANSFER COEFF. TIMES AREA FOR INTERIOR MASS (KW/C

0.900000 FRACTION OF AIR MASS WHICH IS FULLY MIXED

UNIT $24 \quad$ TYPE 2

CONDUIT (DUCT OR PIPE)

1 INPUTS :

FLOW

PRESSURE

TEMPERATURE

TEMPERATURE

TEMPERATURE

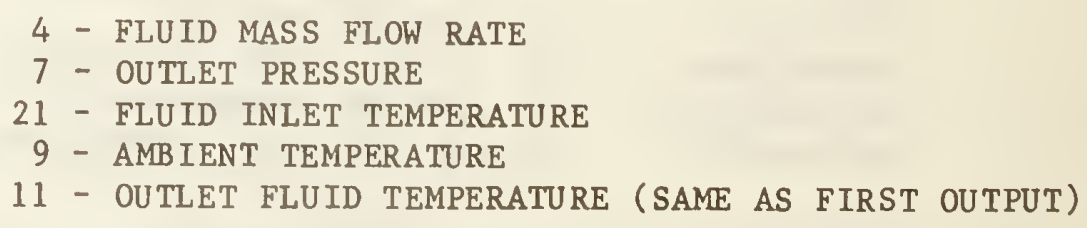

9 - AMBIENT TEMPERATURE

11 - OUTLET FLUID TEMPERATURE (SAME AS FIRST OUTPUT)

2 OUTPUTS :

TEMPERATURE

PRESSURE

11 - OUTLET FLUID TEMPERATURE (SAME AS FIFTH INPUT)

21 - INLET PRESSURE

3 PARAMETERS:

1.00000

0.250000

INSIDE HEAT TRANSFER COEFFICIENT X AREA (KW/C)

OUTSIDE HEAT TRANSFER COEFFICIENT X AREA (KW/C) 


\begin{tabular}{|c|c|c|c|c|}
\hline \multicolumn{3}{|c|}{$\begin{array}{l}220.000 \\
7.75000 \\
0.600000 \mathrm{E}-01 \\
0.000000 \\
1.00000\end{array}$} & \multicolumn{2}{|c|}{$\begin{array}{l}\text { THERMAL CAPACITANCE } \\
\text { VOLUME (M3) } \\
\text { FLOW RESIS TANCE }[0.00 \\
\text { HE IGHT OF OUTLET ABOV } \\
\text { MODE: 2=WATER, } 1=\text { AIR. }\end{array}$} \\
\hline \multicolumn{5}{|c|}{ Initial Variable Values: } \\
\hline PRES SURE & 1 & $\rightarrow$ & 250.000 & $(\mathrm{KPa})$ \\
\hline PRESSURE & 2 & $\rightarrow$ & 227.600 & $(\mathrm{KPa})$ \\
\hline PRESSURE & 3 & $->$ & 212.100 & $(\mathrm{KPa})$ \\
\hline PRESSURE & 4 & $->$ & 222.600 & $(\mathrm{KPa})$ \\
\hline PRESSURE & 5 & $->$ & 210.400 & $(\mathrm{KPa})$ \\
\hline PR ES SURE & 6 & $\rightarrow$ & -250.000 & $(\mathrm{KPa})$ \\
\hline PR ES SURE & 7 & $->$ & 0.399000 & $(\mathrm{KPa})$ \\
\hline PR ESSURE & 8 & $\rightarrow$ & $0.902000 \mathrm{E}-$ & $1(\mathrm{KPa})$ \\
\hline PRES SURE & 9 & $->$ & $0.444000 \mathrm{E}-$ & $1(\mathrm{KPa})$ \\
\hline PRESSURE & 10 & $->$ & $0.176000 \mathrm{E}-$ & $1(\mathrm{KPa})$ \\
\hline PRESSURE & 11 & $->$ & $0.508000 \mathrm{E}-$ & $2(\mathrm{KPa})$ \\
\hline PRESSURE & 12 & $->$ & 0.000000 & $(\mathrm{KPa})$ \\
\hline PRES SURE & 13 & $\rightarrow$ & $-0.537000 \mathrm{E}-$ & $2(\mathrm{KPa})$ \\
\hline PRESSURE & 14 & $\rightarrow$ & $-0.179000 \mathrm{E}-$ & $1(\mathrm{KPa})$ \\
\hline PRESSURE & 15 & $\rightarrow$ & $-0.447000 \mathrm{E}-$ & $1(\mathrm{KPa})$ \\
\hline PRESSURE & 16 & $\rightarrow$ & $-0.288000 \mathrm{E}$ & $3(\mathrm{KPa})$ \\
\hline PRES SURE & 17 & $\rightarrow$ & -0.228000 & $(\mathrm{KPa})$ \\
\hline PRESSURE & 18 & $->$ & -0.283000 & $(\mathrm{KPa})$ \\
\hline PRESSURE & 19 & $\rightarrow$ & -0.338000 & $(\mathrm{KPa})$ \\
\hline PRESSURE & 20 & $\rightarrow$ & 0.151000 & $(\mathrm{KPa})$ \\
\hline PRESSUR E & 21 & $\rightarrow$ & -0.124000 & $(\mathrm{KPa})$ \\
\hline FLOW & 1 & $\rightarrow$ & 1.14200 & $(\mathrm{Kg} / \mathrm{s})$ \\
\hline FLOW & 2 & $\rightarrow$ & 1.49100 & $(\mathrm{Kg} / \mathrm{s})$ \\
\hline FLOW & 3 & $->$ & 2.63300 & $(\mathrm{Kg} / \mathrm{s})$ \\
\hline FLOW & 4 & $\rightarrow$ & 2.14000 & $(\mathrm{Kg} / \mathrm{s})$ \\
\hline FLOW & 5 & $->$ & 0.536000 & $(\mathrm{Kg} / \mathrm{s})$ \\
\hline FLOW & 6 & $\rightarrow$ & 1.60000 & $(\mathrm{Kg} / \mathrm{s})$ \\
\hline TEMPERATURE & 1 & $\rightarrow$ & 5.00000 & (c) \\
\hline TEMPERATURE & 2 & $\rightarrow$ & 5.01300 & (C) \\
\hline TEMPERATURE & 3 & $\rightarrow$ & 16.7900 & (c) \\
\hline TEMPERATURE & 4 & $\rightarrow$ & 32.0000 & (C) \\
\hline TEMPERATURE & 5 & $->$ & 20.0000 & (c) \\
\hline TEMPERATURE & 6 & $\rightarrow$ & 16.8000 & (c) \\
\hline TEMPERATURE & 7 & $\rightarrow$ & 5.00000 & (C) \\
\hline TEM PERATURE & 8 & $\rightarrow$ & 10.1200 & (c) \\
\hline TEMPERATURE & 9 & $->$ & 25.0000 & (C) \\
\hline TEMPERATURE & 10 & $\rightarrow$ & 10.1800 & (C) \\
\hline TEMPERATURE & 11 & $\rightarrow$ & 27.0000 & (c) \\
\hline TEMPERA TURE & 12 & $\rightarrow$ & 28.0000 & (c) \\
\hline TEMPERATURE & 13 & $->$ & 25.0000 & (c) \\
\hline TEMPERATURE & 14 & $\rightarrow$ & 22.0000 & (c) \\
\hline TEMPERATURE & 15 & $->$ & 20.0000 & (C) \\
\hline TEMPERA TUR E & 16 & $->$ & 20.0000 & (c) \\
\hline TEMPERATURE & 17 & $\rightarrow$ & 20.0000 & (C) \\
\hline
\end{tabular}




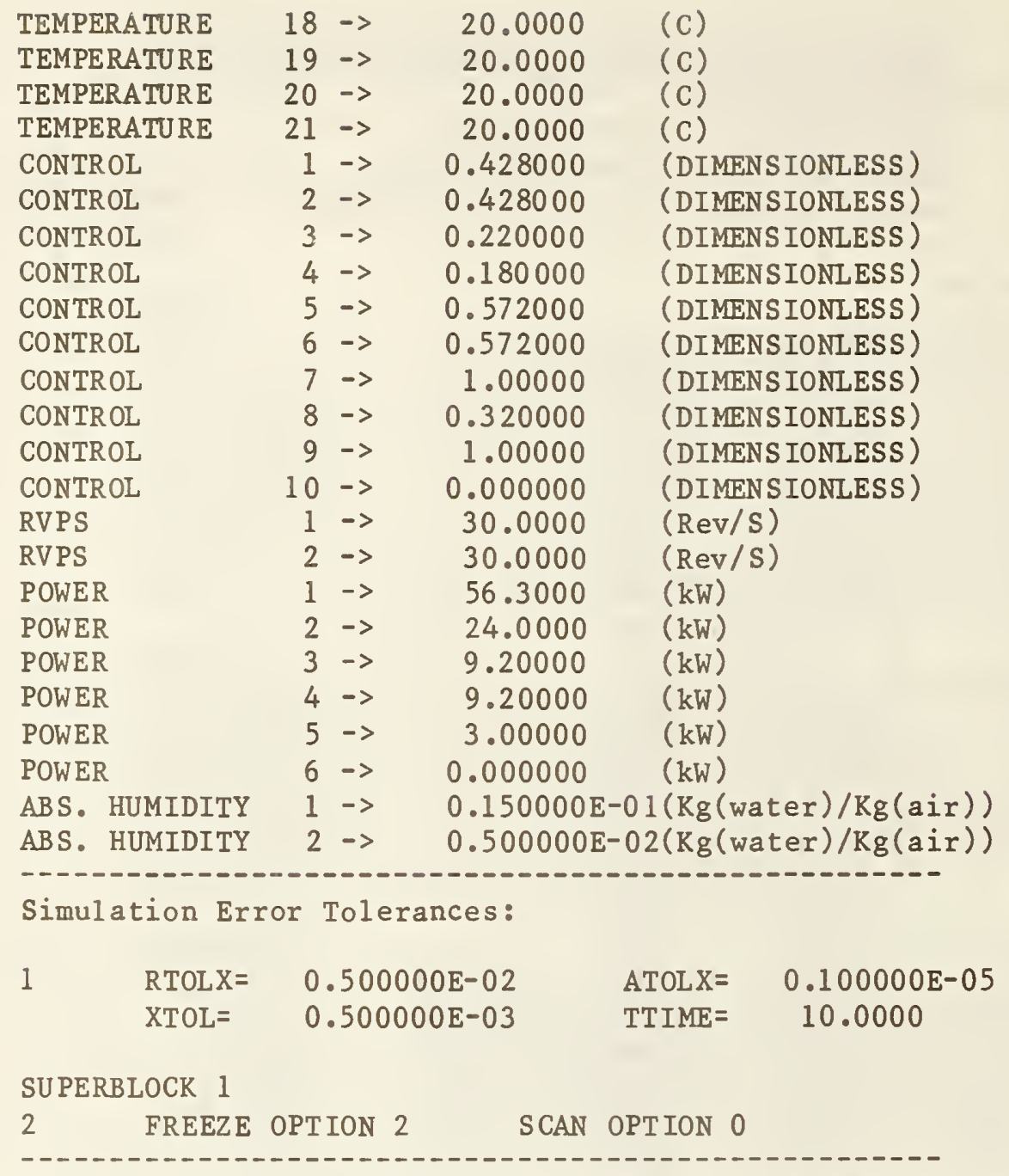

The following are Boundary Variables in the simulation:

The following are the reported variables:

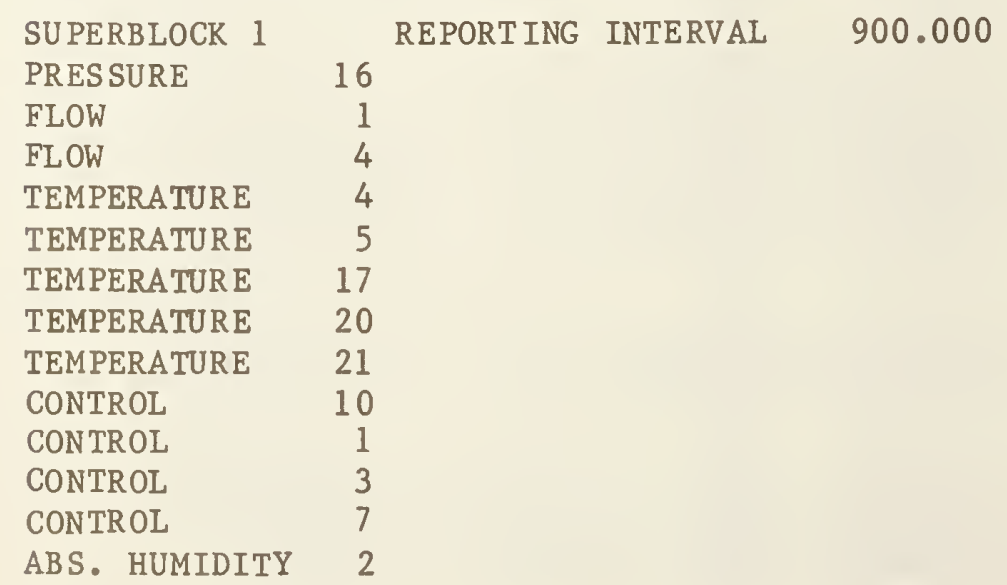


Listing 6.6-2. Boundary Variable File: time, T13, and Q5.

$\begin{array}{rrr}0 & 25.00 & 3.00 \\ 3600 & 25.20 & 3.30 \\ 7200 & 25.70 & 4.05 \\ 10800 & 26.60 & 5.40 \\ 14400 & 27.90 & 7.35 \\ 18000 & 29.40 & 9.60 \\ 21600 & 31.10 & 12.15 \\ 25200 & 32.70 & 14.55 \\ 28800 & 33.90 & 16.35 \\ 32400 & 34.70 & 17.55 \\ 33120 & 34.77 & 17.66 \\ 33840 & 34.84 & 17.77 \\ 34560 & 34.90 & 17.85 \\ 35280 & 34.93 & 17.90 \\ 36000 & 35.00 & 18.00 \\ 39600 & 34.70 & 17.55 \\ 41400 & 34.45 & 17.17 \\ 41760 & 34.38 & 17.07 \\ 42120 & 34.30 & 16.95 \\ 43200 & 34.00 & 16.50 \\ 46800 & 32.90 & 14.85 \\ 50400 & 31.60 & 12.90 \\ 54000 & 30.30 & 10.95 \\ 57600 & 29.20 & 9.30 \\ 61200 & 28.20 & 7.80 \\ 64800 & 27.40 & 6.60 \\ 68400 & 26.80 & 5.70 \\ 72000 & 26.30 & 4.95 \\ 75600 & 25.80 & 4.20 \\ 79200 & 25.40 & 3.60 \\ 82800 & 25.10 & 3.15 \\ 86400 & 25.00 & 3.00 \\ 90000 & 25.20 & 3.30 \\ 93600 & 25.70 & 4.05 \\ 97200 & 26.60 & 5.40\end{array}$




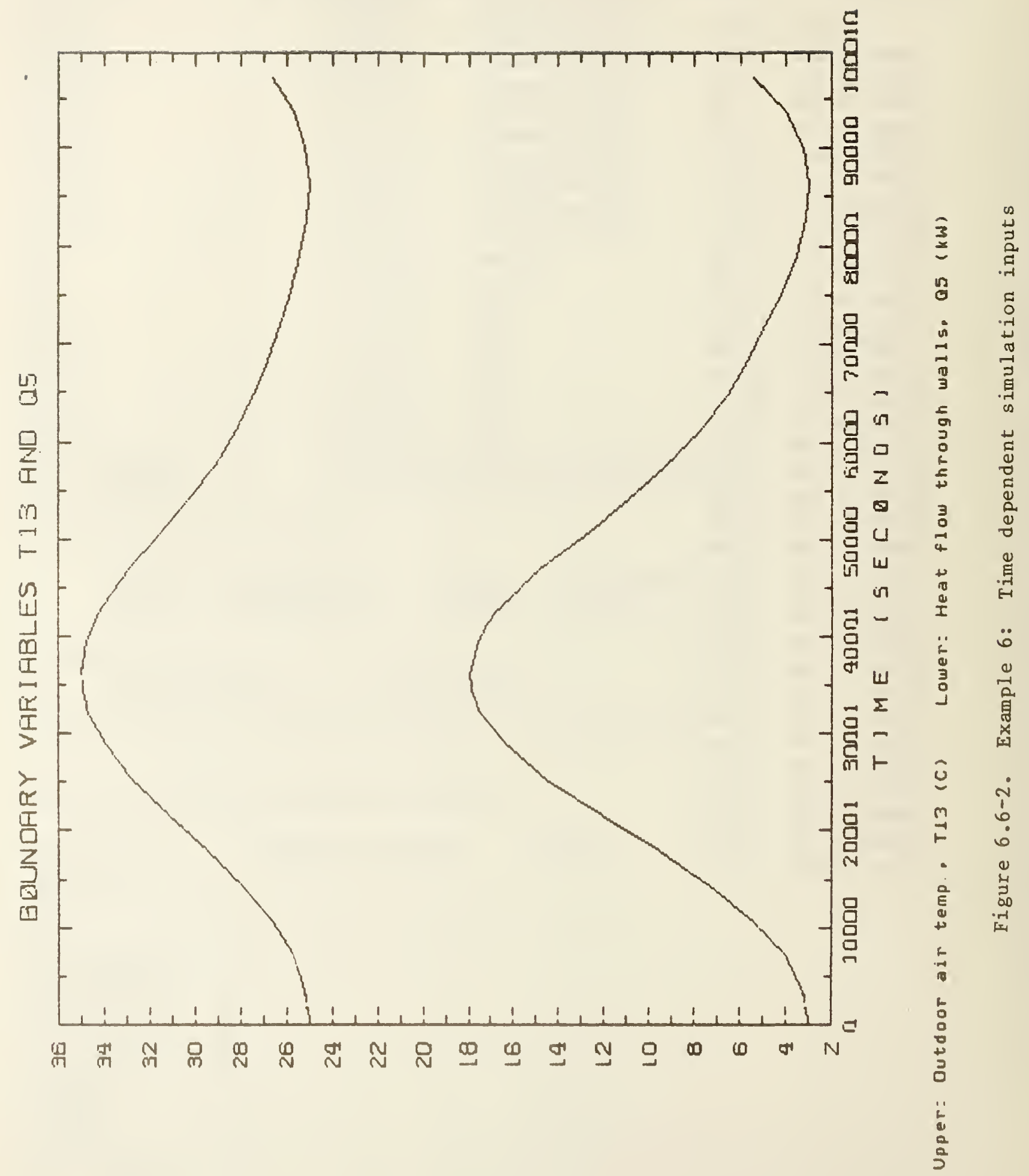




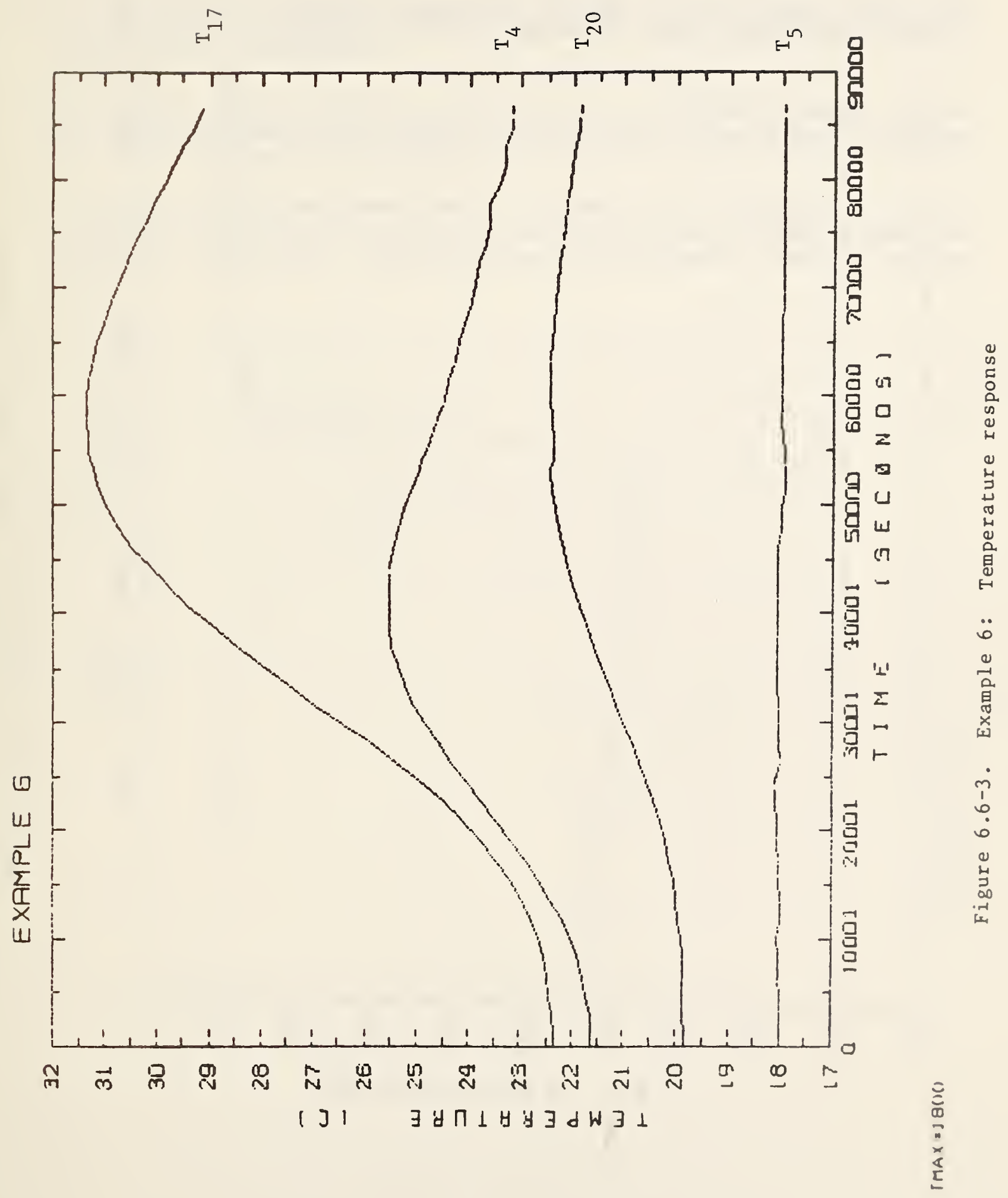




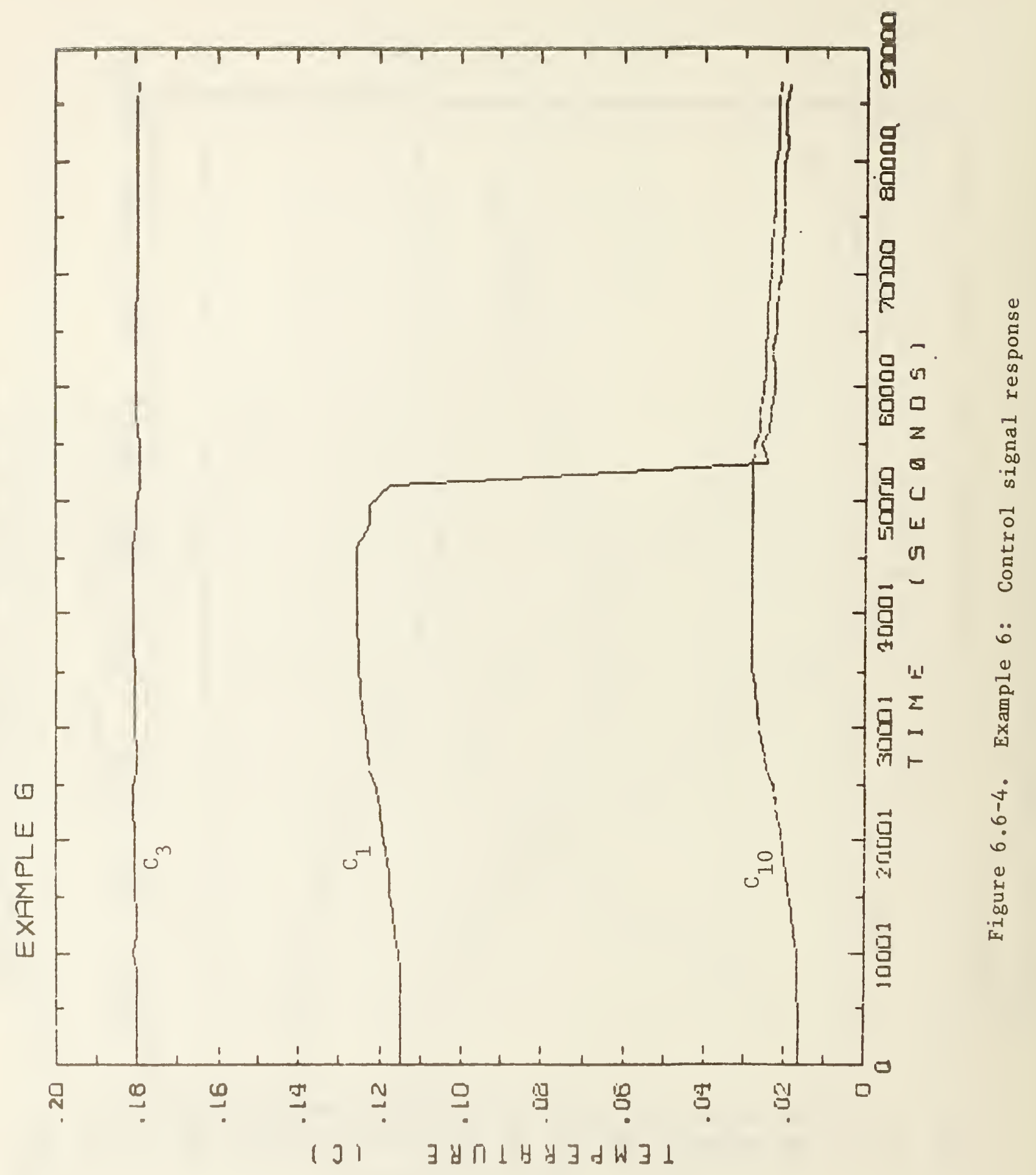


1. Clark, Daniel R. HVACSIM ${ }^{+}$Building Systems and Equipment Simulation Program Reference Manual. National Bureau of Standards, NBSIR 84-2996, January 1985 (110 pages).

2. Park, Cheol; Clark, Daniel R.; Kelly, George E. HVACSIM ${ }^{+}$Building Systems and Equipment Simulation Program: Building Loads Calculation. National Bureau of Standards, NBSIR, 1985.

3. Clark, Daniel R.; Borresen, Bent A. "Dynamics of a Heating Coil Control Loop." Proceedings, Symposium on the Performance of HVAC Systems and Controls in Buildings. Building Research Establishment, Garston, England, 1984. 


\section{APPENDIX}

The following pages provide a summary of the component models supplied with HVACSIM. Brief definitions of each input, output, and parameter are given. The user may need to refer to references [1] and [2] for more detailed descriptions and mathematical definitions.

In the process of setting up simulations, it may be helpful to copy these pages and use them as worksheets. For this reason, only one TYPE component is described on each page, and space has been allowed for filling in unit numbers, input and output index numbers, and parameter values.

The component models summarized in this Appendix are as follows:

TYPE 1 FAN OR PUMP

TYPE 2 CONDUIT (DUCT OR PIPE)

TYPE 3 INLET CONDUIT (DUCT OR PIPE)

TYPE 4 FLOW MERGE

TYPE 5 DAMPER OR VALVE

TYPE 6 FLOW SPLIT

TYPE 7 TEMPERATURE SENSOR

TYPE 8 PROPORT IONAL-INTEGRAL CONTROLLER

TYPE 9 LINEAR VALVE WITH PNEUMATIC ACTUATOR

TYPE 10 HOT WATER COIL MODEL

TYPE 11 HOT WATER TO AIR HEATING COIL

TYPE 12 COOLING OR DEHUMIDIFYING COIL

TYPE 13 THREE-WAY VALVE MODEL

TYPE 14 EVAPORATIVE HUMIDIFIER

TYPE 15 ROOM MODEL

TYPE 16 STICKY PROPORTIONAL CONTROLLER (FOR USE BETWEEN SUPERBLOCKS)

TYPE 17 MIXING DAMPERS AND MERGE

TYPE 18 PLENUM

TYPE 19 FLOW BALANCE CONTROL

TYPE 20 HIGH/LOW LIMIT CONTROLLER

TYPE 21 CLAMPED SPLIT

TYPE 22 STEAM SPRAY HUMIDIFIER

TYPE 23 STEAM NOZZLE

TYPE 24 IDEAL GAS NOZZLE

TYPE 25 STEAM TO AIR HEATING COIL

TYPE 26 CONTROL SIGNAL INVERTER

Types 1-26 are described more fully in reference [1]. Types 27-29 are 1ate additions to the program, described only in this appendix. Types 50-53 are discussed in reference [2]. 


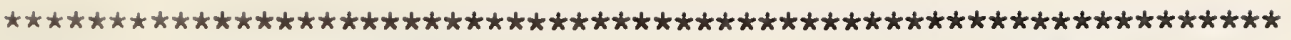
TYPE 1 FAN OR PUMP

$\mathrm{UNIT}=$

\section{IN PUTS :}

MASS FLOW RATE OF FLUID (KG/S)

OUTLET PRESSURE (KPA) -

FAN OR PUMP ROTATIONAL SPEED (REV/S) -

INLET FLUID TEMPERATURE (C) -

OUTPUTS :

INLET PRESSURE (KPA) -

OUTLET FLUID TEMPERATURE (C) -

POWER CONSUMPTION (KW) -

PARAMETERS :

1 1ST PRESSURE COEFFICIENT -

2 2ND PRESSURE COEFFICIENT-

3 3RD PRESSURE COEFFICIENT-

$44 \mathrm{TH}$ PRESSURE COEFFICIENT-

$55 \mathrm{TH}$ PRESSURE COEFFICIENT-

6 1ST EFFICIENCY COEFFICIENT-

7 2ND EFFICIENCY COEFFICIENT-

8 3RD EFFICIENCY COEFFICIENT-

$94 \mathrm{TH}$ EFFICIENCY COEFFICIENT--

105 TH EFFICIENCY COEFFICIENT-

11 DIAMETER (M) -

12 MODE: $A I R=1$, WATER=2 


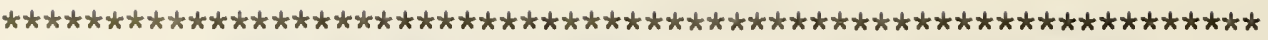
TYPE 2 CONDUIT (DUCT OR PIPE)

UNIT=

INPUTS :

FLUID MASS FLOW RATE (KG/S) OUTLET PRESSURE (KPA) FLUID INLET TEMPERATURE (C) AMBIENT TEMPERATURE (C) OUTLET FLUID TEMPERATURE (SAME AS FIRST OUTPUT) (C) $-\cdots-\cdots-$ T

OUTPUTS :

OUTLET FLUID TEMPERATURE (SAME AS FIFTH INPUT) (C) -

INLET PRESSURE (KPA) -

PARAMETRS :

1 INSIDE HEAT TRANSFER COEFFICIENT X AREA (KW/C)

2 OUTSIDE HEAT TRANSFER COEFFICIENT X AREA (KW/C)---.---.--

3 THERMAL CAPACITANCE OF CONDUIT MATERIAL (KJ/C)--..-

4 VOLJME (M3) -...-

5 FLOW RESISTANCE [0.001/(KG M)]

6 HEIGHT OF OUTLET ABOVE INLET (M)

7 MODE: 2=WATER, 1=AIR, NEG.=DETAILED, POS.=SIMPLE DYNAMICS --- 


\section{INPUTS :}

INLET FLUID PRESSURE (KPA) -

OUTLET FLUID PRESSURE (KPA) -

INLET FLUID TEMPERATURE (C)

AMBIENT AIR TEMPERATURE (C)

OUTLET FLUID TEMPERATURE (SAME AS FIRST OUTPUT) (C)--.-.--T

OUTPUTS :

OUTLET FLUID TEMPERATURE (SAME AS FIFTH INPUT) (C)

FLUID MASS FLOW RATE (KG/S) -

\section{PARAME TERS :}

1 INSIDE HEAT TRANSFER COEFFICIENT X AREA (KW/C)

2 OUTSIDE HEAT TRANSFER COEFFICIENT X AREA (KW/C)

3 THERMAL CAPACITANCE OF CONDUIT MATERIAL (KJ/C)

4 VOLUME (M3) -

5 FLOW RESISTANCE [0.001/(KG M)]

6 HEIGHT OF OUTLET ABOVE INLET (M)

7 MODE: 2=WATER, 1=AIR, NEG.=DETAILED, POS.=SIMPLE DYNAMICS - 


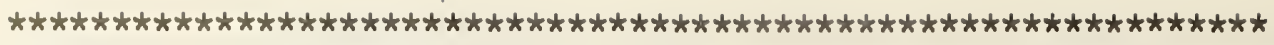
TYPE 4 FLOW MERGE

UNIT $=$

INPUTS :

INLET MASS FLOW RATE 1 (KG/S)

INLET MASS FLOW RATE 2 (KG/S)

OUTLET PRESSURE (KPA)

INLET TEMPERATURE 1 (C)

INLET TEMPERATURE 2 (C)

OUTPUTS :

OUTLET MASS FLOW RATE (KG/S) -

INLET PRESSURE 1 (KPA) -

INLET PRESSURE 2 (KPA)

OUTLET TEMPERATURE (C) -

PARAMETERS :

1 FLOW RESISTANCE $[0.001 /($ KG M) $]$ 


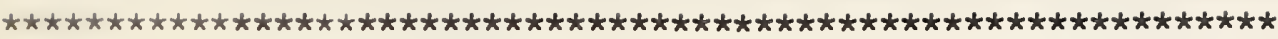

TYPE 5 DAMPER OR VALVE

UNIT $=$

INPUTS :

FLUID MASS FLOW RATE (KG/S)

OUTLET PRESSURE (KPA) -

CONTROL: RELATIVE POSITION OF DAMPER OR VALVE (-)

OUTPUTS :

INLET PRESSURE (KPA) -

PARAMETERS :

1 FLOW RESISTANCE, DAMPER OR VALVE OPEN [0.001/(KG M)]-...-

2 LEAKAGE PARAMETER (DIMENS IONLESS)

3 CHARACTERISTIC: $0=>$ EXP., $1=>$ L IN ., INTERMEDIATE $=>$ INTERMED. - -

4 MODE : $0=>$ CLOSED WHEN CONTROL $=0 ; 1=>$ CLOSED WHEN CONTROL $=1---$ 


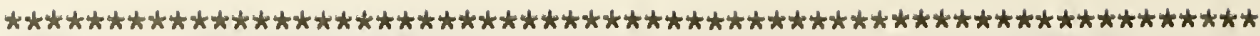

TYPE 6 FLOW SPLIT

UNIT $=$

INPUTS:

INLET MASS FLOW RATE (KG/S)

OUTLET PRESSURE 1 (KPA)

OUTLET PRESSURE 2 (KPA) -

OUTPUTS :

OUTLET MASS FLOW RATE 1 (KG/S)

OUTLET MASS FLOW RATE 2 (KG/S)

INLET PRESSURE (KPA) -

PARAMETERS :

1 FLOW RESISTANCE $[0.001 /(\mathrm{KG}$ M) $]$ 


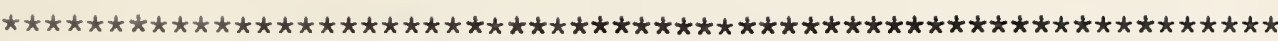

TYPE 7 TEMPERATURE SENSOR

UNIT $=$

IN PUTS :

IN PUT TEMPERATURE (C)

SENSOR OUTPUT (MODIFIED BY GAIN AND OFFSET) (-)

OUTPUTS :

SENSOR OUTPUT (MODIFIED BY GAIN AND OFFSET) (-)

PARAMETERS :

1 SENSOR TIME CONSTANT (SEC)

2 TEMPERATURE OFFSET (C)

3 TEMPERATURE GAIN (C) 


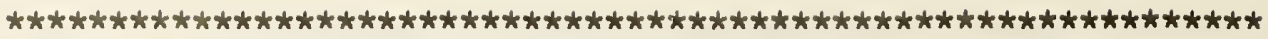

TYPE 8 PROPORTIONAL-INTEGRAL CONTROLLER

UNIT $=$

\section{INPUTS :}

CONTROLLED VARIABLE (-) SET POINT FOR CONTROLLED VARIABLE (-) INTEGRAL PORTION OF CTRL SIGNAL (SAME AS IST OUTPUT) (-)----C OUTPUT CONTROL SIGNAL (SAME AS SECOND OUTPUT) (-) -....--C

\section{OUTPUTS :}

INTEGRAL PORTION OF CONTROL SIGNAL (DIMENSIONLESS) -------C OUTPUT CONTROL SIGNAL (DIMENSIONLESS)

\section{PARAMETERS :}

1 PROPORTIONAL GAIN (DIMENSIONLESS)

2 INTEGRAL GAIN (DIMENSIONLESS)

3 CONTROLLER TIME CONSTANT (SEC) - 


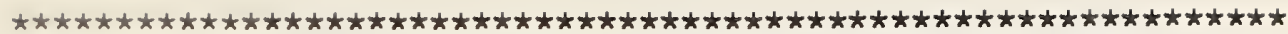
TYPE 9 LINEAR VALVE WITH PNEUMATIC ACTUATOR UNIT=

\section{INPUTS :}

OUTLET WATER PRESSURE (KPA)

WATER MASS FLOW RATE (KG/S)

CONTROL VARIABLE FROM CONTROLLER (DIMENSIONLESS)

ACTUATOR RELATIVE POSITION (SAME AS FIRST OUTPUT) (-) - - ---C

OUTPUTS :

ACTUATOR RELATIVE POSITION (DIMENSIONLESS) -

INLET WATER PRESSURE (KPA)

VALVE STEM RELATIVE POSITION (DIMENSIONLESS) -

PARAMETERS :

1 FLOW RESISTANCE $[0.001 /$ (RG M)]

2 ACTUATOR TIME CONSTANT (SEC)

3 LEAKAGE PARAMETER (DIMENSIONLESS)

4 HYSTERESIS PARAMETER (DIMENSIONLESS) 
INPUTS :

OUTLET AIR PRESSURE (KPA)

AIR MASS FLOW RATE (KG/S)

INLET AIR TEMPERATURE (C)

OUTLET AIR TEMPERATURE (SAME AS FIRST OUTPUT) (C)

OUTLET WATER PRESSURE (KPA)

WATER MASS FLOW RATE (KG/S)

INLET WATER TEMPERATURE (C)

OUTLET WATER TEMPERATURE (SAME AS SECOND OUTPUT) (C) -

OUTPUTS :

OUTLET AIR TEMPERATURE (C)

OUTLET WATER TEMPERATURE (C) -

INLET AIR PRESSURE (KPA)

INLET WATER PRESSURE (KPA)

PARAMETERS :

1 OVERALL HEAT TRANSFER COEFFICIENT TIMES AREA (KW/C) -

2 FLOW RESISTANCE PARAMETER, AIR SIDE [0.001/(KG M)]

3 FLOW RESISTANCE PARAMETER, WATER SIDE [0.001/(KG M)]--.-

4 WATER SIDE VOLUME OF COIL (M3)-

5 CAPACITIVE TERM OF COIL TIME CONSTANT (SEC) 


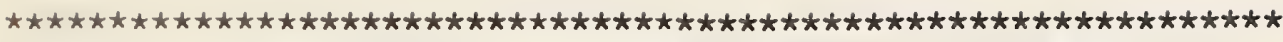
TYPE 11 HOT WATER TO AIR HEATING COIL UNIT=

INPUTS :

OUTLET AIR PRESSURE (KPA)

AIR MASS FLOW RATE (KG/S)

INLET AIR TEMPERATURE (C) -

OUTLET AIR TEMPERATURE (SAME AS FIRST OUTPUT) (C) -

OUTLET WATER PRESSURE (KPA) -

WATER MASS FLOW RATE (KG/S)

INLET WATER TEMPERATURE (C) -

OUTLET WATER TEMPERATURE (SAME AS SECOND OUTPUT) (C)--.----T

DUMMY TEMPERATURE (SAME AS THIRD OUTPUT) (C) -

OUTPUTS :

OUTLET AIR TEMPERATURE (C) -

OUTLET WATER TEMPERATURE (C)

DUMMY TEMP. (USED INTERNALLY FOR AIR TEMP. DYNAMICS) (C)-----T

INLET AIR PRESSURE (KPA) -

INLET WATER PRESSURE (KPA)

PARAMETERS :

1 COEFFICIENT FOR AIR SIDE HEAT TRANSFER CORRELATION (-)----

2 EXPONENT FOR AIR SIDE HEAT TRANSFER CORRELATION (-) -

3 COEFFICIENT FOR WATER SIDE HEAT TRANSFER CORRELATION (-)---

4 EXPONENT FOR WATER SIDE HEAT TRANSFER CORRELATION (-) ---

5 AIR FLOW RESISTANCE PARAMETER [0.001/(KG M)]

6 WATER FLOW RESISTANCE PARAMETER $[0.001 /($ KG M)]

7 WATER SIDE VOLUME OF COIL (M3) -

8 CAPACITANCE (MASS X SPECIFIC HEAT) OF COIL MATERIAL (KJ/C) - 


\section{INPUTS :}

WATER MASS FLOW RATE (KG/S) INLET WATER TEMPERATURE (C) DRY AIR MASS FLOW RATE (KG/S) INLET DRY BULB AIR TEMPERATURE (C) INLET AIR HUMIDITY RATIO (DIMENSIONLESS) OUTLET WATER TEMPERATURE (SAME AS FIRST OUTPUT) (C) -....--T OUTLET AIR TEMPERATURE (SAME AS SECOND OUTPUT) (C) -....--T OUTLET AIR HUMIDITY RATIO (SAME AS THIRD OUTPUT) (-) - - - - -

\section{OUTPUTS :}

OUTLET WATER TEMPERATURE (C) OUTLET AIR DRY BULB TEMPERATURE (C) OUTLET AIR HUMIDITY RATIO (DIMENSIONLESS) TOTAL COOLING LOAD (KW) SENSIBLE COOLING LOAD (KW) WET FRACTION OF COIL SURFACE AREA (DIMENSIONLESS) -.-.-.-C

\section{PARAMETERS :}

1 COIL TYPE: 0=FLAT CONTINUOUS FINS, 1=CIRCULAR FINS--.----

2 PRIMARY (TUBE EXTERIOR) SURFACE AREA (M2)

3 SECONDARY (FIN) SURFACE AREA (M2)

4 INTERNAL SURFACE AREA (M2)

5 RATIO OF MINIMUM AIR FLOW AREA TO FACE AREA (-)

6 FIN MATERIAL THERMAL CONDUCTIVITY $[\mathrm{KW} /(\mathrm{M} \mathrm{R})]$

7 COIL FACE AREA (M2) -

8 NUMBER OF FINS PER CENT IMETER-_.

9 NUMBER OF TUBES PER ROW -

10 NUMBER OF ROWS -

11 OUTSIDE TUBE DIAMETER (M)

12 INSIDE TUBE DIAMETER (M)

13 FIN THICKNESS (M)

14 THERMAL CAPACITANCE (MASS X SPECIFIC HEAT) OF COIL (KJ/K)-

15 TUBE ROW SPACING IN AIR FLOW DIRECTION (M) -...-

16 FIN DIAMETER [IF PAR(1)=1] OR LENGTH [IF $\operatorname{PAR}(1)=0](M)---$

17 COIL DEPTH IN AIR FLOW DIRECTION (M)

18 TUBE THERMAL CONDUCTIVITY [KW/(M K)] 


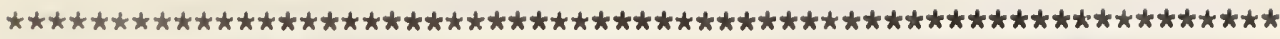

TYPE 13 THREE-WAY VALVE MODEL

UNIT=

\section{INPUTS :}

INLET MASS FLOW RATE, PORT 1 (OPEN WHEN CONTROL = 1) $(\mathrm{KG} / \mathrm{S})-\mathrm{M}$ INLET MASS FLOW RATE, PORT 2 (OPEN WHEN CONTROL = 0) $(\mathrm{KG} / \mathrm{S})-\mathrm{M}$ OUTLET PRESSURE (KPA) INLET TEMPERATURE, PORT 1 (C) INLET TEMPERATURE, PORT 2 (C) CONTROL VARIABLE FROM CONTROLLER (-) ACTUATOR RELATIVE POSITION (SAME AS OUTPUT 1) (-)

OUTPUTS :

ACTUATOR RELATIVE POSITION (-) OUTLET MASS FLOWRATE (KG/S) INLET PRESS., PORT 1 (OPEN $\Rightarrow$ CONTROL VARIABLE $=1$ ) $(\mathrm{KPA})----\mathrm{P}$ INLET PRESS., PORT 2 (OPEN $\Rightarrow$ CONTROL VARIABLE $=0$ ) $(\mathrm{KPA})----\mathrm{P}$ OUTLET TEMPERATURE (C) VALVE STEM RELATIVE POSITION (-)

PARAMETERS :

1 PORT 1 FLOW RESISTANCE, PORT 1 OPEN (CTRL=1) [0.001/(KG M)] -

2 PORT 2 FLOW RESISTANCE, PORT 2 OPEN (CTRL=0) $[0.001 /(\mathrm{KG} \mathrm{M})]$ -

3 LEAKAGE PARAMETER (DIMENSIONLESS) -

4 ACTUATOR TIME CONSTANT (SEC) -

5 HYSTERESIS PARAMETER (DIMENSIONLESS)

6 PORTS 1,2 MODE: $0=>\mathrm{LIN} / \mathrm{LIN}, 1=>\mathrm{EXP} / \mathrm{L}$ IN, ELSE $=>\mathrm{EXP} / \mathrm{EXP}$ 


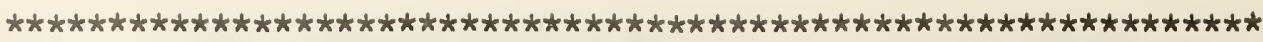
TYPE 14 EVAPORATIVE HUMIDIFIER

UNIT $=$

INPUTS :

INLET AIR TEMPERATURE (C)

INLET AIR HUMIDITY RATIO (DIMENSIONLESS)

INLET MOIST AIR MASS FLOW RATE (KG/S)

OUTPUTS :

OUTLET AIR HUMIDITY RATIO (DIMENSIONLESS)--

OUTLET AIR TEMPERATURE (C)

OUTLET MOIST AIR MASS FLOW RATE (KG/S)

PARAMETERS :

1 WATER TO AIR HEAT TRANSFER COEFFICIENT TIMES AREA (C)--.-- 


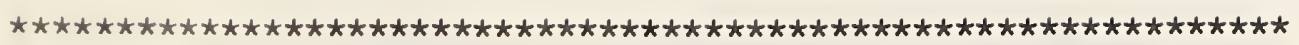

TYPE 15 ROOM MODEL

UNIT=

IN PUTS :

MASS FLOW RATE OF VENTILATION AIR (KG/S) VENTILATION AIR INLET TEMPERATURE (C) TEMP. . FULLY MIXED PART OF AIR MASS [SAME AS OUT(1)] (C)----T WALL MASS TEMPERATURE (SAME AS SECOND OUTPUT) (C)--.--INTERIOR MASS TEMPERATURE (SAME AS THIRD OUTPUT) (C)---.-- T SPATIAL AVG. TEMP., PISTON FLOW AIR MASS (OUTPUT 4) (C)------T CONDUCTION HEAT FLOW INTO WALL MASS (KW) HEAT FLOW DUE TO INTERNAL GAINS (KW)

OUTPUTS :

TEMPERATURE OF FULLY MIXED PORTION OF AIR MASS (C)---1--- T WALL MASS TEMPERATURE (C) INTERIOR MASS TEMPERATURE (C) SPATIAL AVG. TEMP.. PISTON FLOW PORTION OF AIR MASS (C)------T AVERAGE ROOM AIR TEMPERATURE (C)

EXHAUST AIR TEMPERATURE (C)

PARAMETERS :

1 VOLUME OF ROOM AIR MASS (M3)

2 THERMAL CAPACITANCE OF WALLS (KJ/C)

3 THERMAL CAPACITANCE OF INTERIOR MASS (KJ/C)

4 HEAT TRANSFER COEFF. TIMES AREA FOR WALL MASS (KW/C)-..--

5 HEAT TRANSFER COEFF. TIMES AREA FOR INTERIOR MASS (KW/C)---

6 FRACTION OF AIR MASS WHICH IS FULLY MIXED 


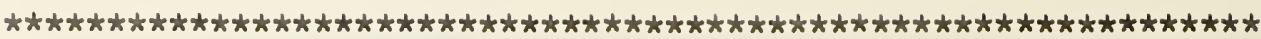

TYPE 16 STICKY PROPORTIONAL CONTROLLER

UNIT=

(FOR USE BETWEEN SUPERBLOCKS)

INPUTS :

MEASURED CONTROLLED VARIABLE (DIMENSIONLESS) -

CONTROLLED VARIABLE SETPOINT (DIMENSIONLESS) -

OUTPUTS :

CONTROLLER OUTPUT SIGNAL（DIMENSIONLESS） -

PARAMETERS :

1 PROPORTIONAL GAIN (DIMENSIONLESS)

2 ERROR BAND (DIMENS IONLESS)

3 OFFSET (DIMENSIONLESS) 


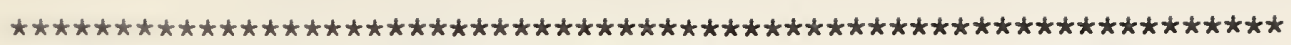

TYPE 17 MIXING DAMPERS AND MERGE

UNIT $=$

INPUTS :

PRESSURE, INLET 1 (CTRL $=0 \Rightarrow$ DAMPER CLOSED) (KPA)------- P

PRESSURE, INLET 2 (CTRL = $0 \Rightarrow$ DAMPER OPEN) (KPA) -

OUTLET PRESSURE (KPA) -

TEMPERATURE AT INLET 1 (CTRL $=0 \Rightarrow$ DAMPER CLOSED) (C)---.--T

TEMPERATURE AT INLET 2 (CTRL $=0 \Rightarrow$ DAMPER OPEN) (C)

INPUT CONTROL SIGNAL: RELATIVE DAMPER POSITION (-)-.-.-.--C

OUTPUTS :

MASS FLOW RATE, INLET 1 (CTRL $=0 \Rightarrow$ DAMPER CLOSED) (KG/S) -- M

MASS FLOW RATE, INLET 2 (CTRL $=0 \Rightarrow$ DAMPER OPEN) (KG/S) - - - M

OUTLET MASS FLOW RATE (KG/S)

OUTLET TEMPERATURE (C)

PARAME TERS :

1 FLOW RESISTANCE WHEN DAMPER IS OPEN [0.001/(KG M)]

2 LEAKAGE PARAMETER (DIMENS IONLESS) -

3 CHARACTERISTIC: $0=>$ EXP., $1=>$ LIN . , INTERMEDIATE $=>$ INTERMED . - - 


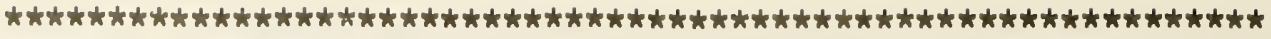

TYPE 18 PLENUM

UNIT=

INPUTS:

INLET MASS FLOW RATE 1 (KG/S) $\cdots \ldots \ldots-n$

INLET MASS FLOW RATE 2 (KG/S)

INLET MASS FLOW RATE 3 (KG/S)

INLET MASS FLOW RATE 4 (KG/S)

INLET MASS FLOW RATE 5 (KG/S) -

INLET MASS FLOW RATE 6 (KG/S)

INLET MASS FLOW RATE 7 (KG/S) -

INLET MASS FLOW RATE 8 (KG/S)

INLET MASS FLOW RATE 9 (KG/S)

INLET MASS FLOW RATE 10 (KG/S)

OUTPUTS :

OUTLET MASS FLOWRATE (KG/S) -

PARAMETERS :

1 NUMBER OF FLOW STREAMS MERGING (DIMENSIONLESS)--...-..- 


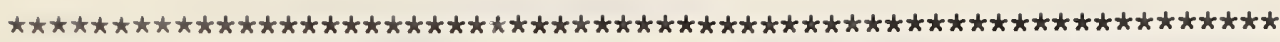
TYPE 19 FLOW BALANCE CONTROL

UNIT $=$

IN PUTS :

MASS FLOW RATE DETERMINED IN THIS SUPERBLOCK (KG/S) -

PRESSURE WHERE FLOW ENTERS THIS SUPERBLOCK (KPA)-......-P

MASS FLOW RATE DETERMINED IN ANOTHER SUPERBLOCK (KG/S) .......

OUTPUTS :

PRESSURE WHERE FLOW EXITS THIS SUPERBLOCK (KPA)

PARAMETERS :

1 INITIAL FLOW RESISTANCE PARAM. AT TIME $=0[0.001 /(\mathrm{KG} \mathrm{M})]-$ - 


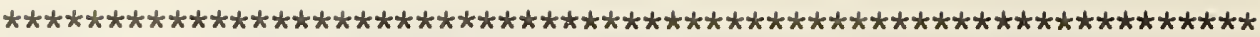

TYPE 20 HIGH/LOW LIMIT CONTROLLER

UNIT $=$

INPUTS :

MEASURED TEMPERATURE INPUT CONTROL SIGNAL (-)

CONTROLLER OUTPUT (SAME AS OUTPUT 1) (-)

OUTPUTS:

CONTROLLER OUTPUT (-)

PARAMETERS:

1 PROPORTIONAL GAIN -...

2 SETPOINT TIMES I, WHERE I=1 (HIGH LIM.) OR I=-1 (LOW LIM.)- 
ス

TYPE 21 CLALPED SPLII

UIII $=$

\section{IITTS :}

PRESSURE AI OUILEI 1 (RPA) $\cdots \ldots \ldots \ldots$

PRESSLBE AI OUILEI 2 (RPA) -

OUIPUTS :

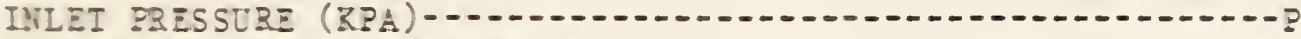

MASS ILOH RAIE, IILEI (RG/S) -

MASS ILOF RATE, OUTIET I (KG/S)

ASS IIOW RATE, OUTIET 2 (RG/S)

PARALIETER:

1 PRESSURE AT CELIER NODE OE SEIIT (RPA) -

2 ILOH RESISTALE RARALEIIR $[0.001 /($ KG H) $] \ldots \ldots \ldots$ 


\section{INPUTS :}

STEAM TEMPERATURE (C)

INLET AIR TEMPERATURE (C) $-100-1$

INLET AIR HUMIDITY RATIO (-)

INLET MOIST AIR FLOW RATE (KG/S)

STEAM FLOW RATE (KG/S)

OUTPUTS :

OUTLET AIR TEMPERATURE (C)

OUTLET MOIST AIR FLOW RATE (KG/S)

OUTLET AIR HUMIDITY RATIO (-)

PARAMETERS :

1 SATURATION EFFICIENCY (-) 


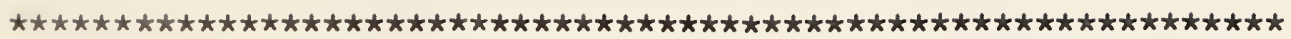

TYPE 23 STEAM NOZZLE

UNIT=

INPUTS :

ENTERING STEAM TEMPERATURE (C)

STEAM STAGNATION PRESSURE AT ENTRANCE (KPA)-

EXIT PRESSURE (KPA) -

OUTPUTS :

OUTLET STEAM TEMPERATURE (C)

MASS FLOW RATE OF STEAM (KG/S)

PARAMETERS :

1 NOZZLE EXIT CROSS SECTIONAL AREA (M2) 


\section{INPUTS :}

UPSTREAM STAGNATION PRESSURE (KPA, GAGE)

EXIT PRESSURE (KPA, GAGE)

UPS TREAM TEMPERATURE (C)

OUTPUTS :

MASS FLOW RATE OF GAS (KG/S)

EXIT TEMPERATURE (C)

PARAMETERS :

1 NOZZLE EXIT CROSS SECTIONAL AREA (M2)

2 ABS. PRESSURE CORRESPONDING TO ZERO GAGE PRESSURE (KPA)----

3 RATIO OF SPECIFIC HEATS (DIMENSIONLESS)

4 GAS CONSTANT [J/(KG K)] 


\section{INPUTS :}

STEAM PRESSURE (KPA)

INLET STEAM TEMPERATURE (C)

INLET AIR TEMPERATURE (C)

OUTLET AIR TEMPERATURE (FROM OUTPUT 1) (C)

MASS FLOW RATE OF AIR (KG/S)

OUTPUTS :

OUTLET AIR TEMPERATURE (C)

STEAM OR WATER OUTLET TEMPERATURE (C)

STEAM AND CONDENSATE MASS FLOW RATE (KG/S)

RATE OF HEAT TRANSFER TO AIR (KW)

\section{PARAMETERS :}

1 DEGREES OF SUBCOOLING OF CONDENSATE (C)

2 MINIMUM AIR FLOW AREA (M2)

3 TUBE INSIDE SURFACE AREA FOR HEAT TRANSFER (M2)

4 TUBE OUTSIDE SURFACE AREA FOR HEAT TRANSFER (M2)--...--

5 FIN SURFACE AREA FOR HEAT TRANSFER (M2) -

6 TUBE INSIDE DIAMETER (M)

7 TUBE OUTSIDE DIAMETER (M)

8 TUBE THERMAL CONDUCTIVITY $[\mathrm{W} /(\mathrm{M} \mathrm{K})]$

9 FIN DIAMETER (M) -

10 FIN MATERIAL THERMAL CONDUCTIVITY [W/(M K)]

11 FIN THICKNESS (M) -

12 NUMBER OF FINS PER METER--

13 TOTAL THERMAL CAPACITANCE OF FINS AND TUBES (KJ/C) 


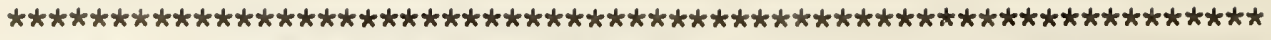

TYPE 26 CONTROL SIGNAL INVERTER UNIT=

INPUTS :

INPUT CONTROL SIGNAL (-)

OUTPUTS :

OUTPUT CONTROL SIGNAL (-)

PARAMETERS : NONE 


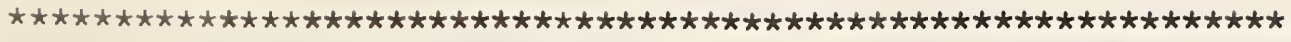
TYPE 27 MOIST AIR FLOW MERGE

$\mathrm{UNIT}=$

\section{IN PU TS :}

INLET MASS FLOW RATE 1 (KG/S)

INLET MASS FLOW RATE 2 (KG/S)

OUTLET PRESSURE (KPA) -

INLET TEMPERATURE 1 (C)

INLET TEMPERATURE 2 (C)

INLET HUMIDITY RATIO 1 (-)

INLET HUMIDITY RATIO 2 (-)

OUTPUTS :

OUILET MASS FLOW RATE (KG/S)

INLET PRESSURE 1 (KPA)

INLET PRESSURE 2 (KPA)

OUTLET TEMPERATURE (C)

OUTLET HUMIDITY RATIO (-) -

\section{PARAMETERS :}

1 FLOW RESISTANCE $[0.001 /(\mathrm{KG}$ M) ] 


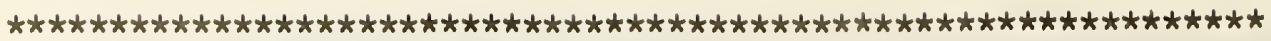

TYPE 28 CONSTANT FLOW RESISTANCE

UNIT =

INPUTS :

FLUID MASS FLOW RATE (KG/S)

OUTLET PRESSURE (KPA)

OUTPUTS :

INLET PRESSURE (KPA)

PARAMETERS :

1 FLOW RESISTANCE $[0.001 /($ KG M) $]$ 


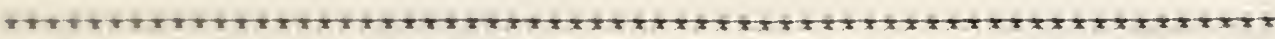

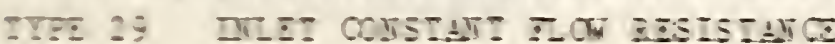

Thit:=

Di:m:

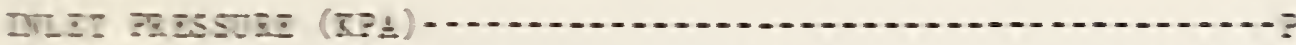

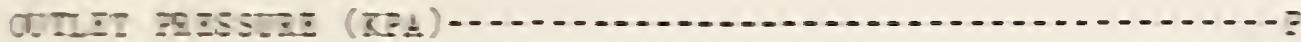

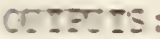

IIIT) WLSS IL

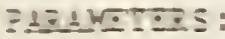

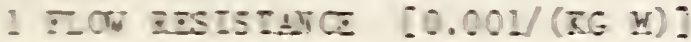




\section{IN PUTS :}

TIA: Zone air dry-bulb temperature (C) -

QISW: Internal (short wave) radiant gain from lights ( $k W)--Q$

QILW: Internal (long wave) radiant gain ( $\mathrm{kW}$ )

TIS(1): Inner surface temperature (C)

TIS(2): Inner surface temperature (C)

TIS(3): Inner surface temperature (C)

TIS (4): Inner surface temperature (C)

TIS(5): Inner surface temperature (C)

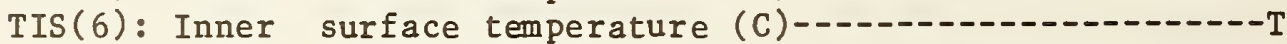

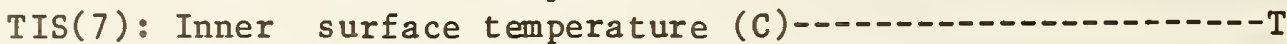

TIS(8): Inner surface temperature (C)-n-.-.-.

TIS(9): Inner surface temperature (C)--.-.-.-.

TIS(10):Inner surface temperature (C)

\section{OUTPUTS :}

TMR: Mean radiant temperature (C)

QWALL: Convective heat gain from building surfaces (kW)-----Q

\section{PARAMETERS :}

1 IZN: Identification number of zone-n

2 NS: Number of surfaces of zone-

NOTE: when the number of surfaces is less than 10, an index of zero may be used for the remaining temperature inputs. 


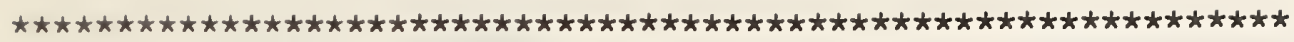

TYPE 51 SURFACE CONSTRUCT

UNIT $=$

\section{INPUTS :}

TIA: Indoor air dry-bulb temperature (C) -

TMR: Mean radiant temperature (C) -

TOSINF: Outer surface temp. of unexposed wall (C) -

FSHADW: Shaded fraction of exposed outer surface (-) -..--.-C

\section{OUTPUTS :}

TIS: Inner surface temperature (C) -

SOLINT: Integrated solar flux incident on surface (W/m2)-----Q

\section{PARAME TERS :}

1 IZN: Identification number of zone--

2 ID: Identification number of surface-1.

3 IEXPOS: $0=W /$ in $z$ one, $1=$ betw.zones, $2=$ exposed to sun (-)----

4 ISTR: Identification number of the construct--.-

5 AS: Surface area $(m * \star 2)-1$

6 ORIENT: Azimuth angle between normal to surface \& south----

7 TILT: Slope of surface: flat roof $=0$, floor $=180$ (degree)--

$8 \mathrm{GRF}$ : Ground reflectivity (-) -

9 IROFS: Outer surface roughness index $[1,6](-)$

10 ABSOS: Solar absorptance of the outer surface $(-)$

11 ABSIS: Short wave absorptance of the inner surface (-) --

12 EMITIS: Emissivity of the inner surface (-)

13 TRANSM: Transmittance of the glass window (-)

14 SC: Shading coefficient of the glass window (-) --.--

NOTES: If outer surface is exposed to outside air (IEXPOS=2), TOSINF may be given an index of zero.

The orientation angle of a west facing surface is 90 degrees, while an east facing surface is 270 degrees.

The surface roughness index, IROFS, is defined as follows:

IROFS $=1 \Rightarrow$ Stucco

IROFS $=2 \Rightarrow$ Brick, rough plaster

IROFS $=3 \Rightarrow$ Concrete

IROFS $=4 \Rightarrow$ Clear pine

IROFS $=5 \Rightarrow$ Smooth plaster

IROFS $=6 \Rightarrow$ Glass, paint on pine 


\section{INPUTS :}

PIAG: Gage pressure of zone air ( $\mathrm{kPa}$ )

TIA: Zone air dry-bulb temperature (C)

WIA: Humidity ratio of zone air (-)

PSAG: Gage pressure of supply air $(\mathrm{kPa})$

MSA: Mass flow rate of supply air $(\mathrm{kg} / \mathrm{s})$

TSA: Supply air dry-bulb temperature (C) -

WSA: Humidity ratio of supply air (-)

QWALL: Convective heat gain from building surfaces (kW)-----Q

NUMPEP: Number of people (occupant in the zone)

UTCEQP: Equipment utilization coefficient (-)

UTCLIT: Lighting utilization coefficient (-) -

\section{OUTPUTS :}

TIA: Zone air dry bulb temperature [diff.eq.] (C) --------T

WIA: Humidity ratio of zone air (-) [diff.eq.] (-) ------H

QISW: Internal (short wave) radiant gain from lights (kW)--Q

QILW: Internal (long wave) radiant gain ( $\mathrm{kW}$ )

PARAMETERS:

1 IZN: Identification number of zone-1

2 CFUR: Effective thermal capacitance of furnishings (kJ/K)-

3 EFFMIA: Air mass multiplier for zone moisture capacitance---

4 VOLUME: Volume of zone air (interior space of zone) ( $m * * 3$ )--

5 SAIREX: Infiltration Coef. (.5=tight,1.=std,1.5=leaky) --.--

6 WPEPS: Sensible heat gain from a person (kW)

7 WPEPL: Latent heat gain from a person (kW)

8 WLIT: Heat gain due to lighting in the zone $(\mathrm{kW})$

9 LIGHT: 1 = Fluorescent, 2 = Incandescent (-)

10 WEQPS: Sensible heat gain due to equipment (kW)

11 WEQPL: Latent heat gain due to equipment (kW)

12 REQP: Radiative to sensible heat from equipment (-) - - - 


\section{INPUTS :}

TAMB: Ambient (outdoor) air temperature (C)

HUMRAT: Outdoor air humidity ratio $(-)$

PBAR: Barometric pressure $(\mathrm{kPa})$

IDN: Direct normal solar radiation (W/m*m)

ISKY: Diffuse (sky) solar radiation (W/m*m)

IHOR: Total horizontal solar radiation (W/m*m)

OUTPUTS : NONE

\section{PARAMETERS :}

1 Index for ambient temperature (e.g. 5 if $\mathrm{TAMB}=\mathrm{T} 5$ )

2 Index for outdoor air humidity ratio

3 Index for barometric pressure

4 Index for direct normal solar radiation

5 Index for diffuse (sky) solar radiation

6 Index for total horizontal solar radiation 
NBS.I1 $\angle A$ (REV. 3-BC)

U.S. DEPT. OF COMM.

BIBLIOGRAPHIC DATA

SHEET (See instructions)

1. PUBLICATION OR
REPORT NO.

NBSIR 85-3243

2. Performing Organ. Report Nod 3. Publlcation Date

4. TITLE AND SUBTITLE

HVACSIM $^{+}$BUILDING SYSTEMS AND EQUIPMENT SIMULATION PROGRAM - USERS GUIDE

\section{5. $\operatorname{AUTHOR}(S)$}

Daniel R. Clark and William B. May, Jr.

6. PERFORMING ORGANIZATION (If joint or other than NBS, see instructions)

NATIONAL BUREAU OF STANDARDS

DEPARTMENT OF COMMERCE

WASHINGTON, D.C. 20234

\section{SPONSOAINC ORGAHIZATION NAME AND COMFLETE ADDRESS (Street, City, StOte, ZIP)}

Office of Buildings \& Community Systems U.S. Naval Civil Engineering Laboratory

U.S. Department of Energy

1000 Independence Ave., NW

U.S. Department of Defense

Washington, DC 20585

10. SUPPLEMENTARY NOTES

Document describes a computer program; SF-185, FIPS Software Summary, is attached.

11. ABSTRACT (A 200-word or less foctual summary of most significant information. If document includes o significant bibliogrophy or literoture survey, mention it here)

HVACSIM' is a modular, non-proprietary computer simulation package developed at the National Bureau of Standards. The package consists of a general-purpose modular simulation program called MODSIM, a library of component models specific to building systems, and a simulation editor called HVACGEN. The latter is used to facilitate the creation and modification of simulation descriptions. HVACSIMt is designed to allow detailed simulation of entire building systems or portions of such systems. This includes the heating, ventilating, and air conditioning (HVAC) system, the equipment control system, the conditioned zones within a building, the building shell, and the dynamic interactions among these subsystems.

This document describes the procedures for installing HVACSIM on a particular computer, for setting up a simulation description using HVACGEN, and for running a simulation using MODSIM. A number of example simulations are included to illustrate the procedures and to serve as a tutorial in the use of HVACSIMt. A Reference Manual, published separately, describes the main simulation program and the library of component models in more detail. A third document, also published separately, describes the building model supplied with HVACSIM+.

12. KEY WORDS (Six to twelve entries; alphabetical crder: copitolize only proper names: and separate key words by semicolons) building dynamics; building simulation; bullding system modeling; computer simulation programs; control dynamics; dynamic modeling of building systems; dynamic performance of building systems; dynamic simulations: HVAC system simulat1ons: HVACSIM ${ }^{+}$

13. AVAILABILITY

[X] Unlimited

ए For Official Distribution. Do Not Release to NTIS

$\square$ Order From Superintendent of Documents, U.S. Government Printing Office, Washington, D.C. 20402.

[X] Order From National Technical Information Service (NTIS), Springfield, VA. 2216I
14. NO. OF PRINTED PAGES

202

15. Price 
